\title{
New fatty acids, oxylipins and volatiles in microalgae
}

Dissertation zur Erlangung des Doktorgrades der Mathematisch-Naturwissenschaftlichen Fakultäten der Georg-August-Universität zu Göttingen

\author{
vorgelegt von \\ Imke Lang \\ aus Oldenburg
}

Göttingen 2007 
Referent: $\quad$ Prof. Dr. Ivo Feußner

Korreferent: $\quad$ Prof. Dr. Thomas Friedl

Tag der Disputation: 24.08. 2007 


\section{Index}

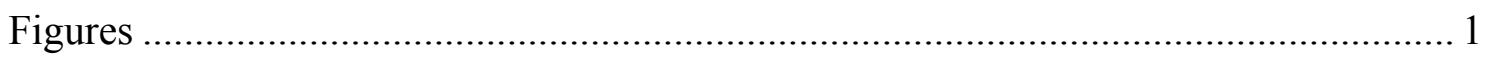

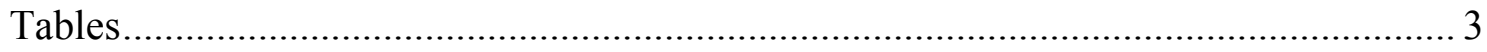

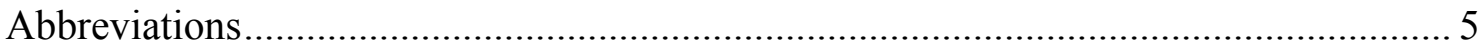

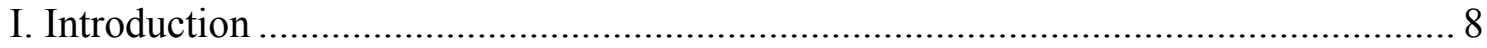

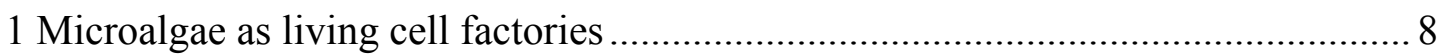

1.3 Microalgae as a source of polyunsaturated fatty acids (PUFAs) ...................... 9

1.2 Screening for new FAs and lipids from microalgae ..................................... 10

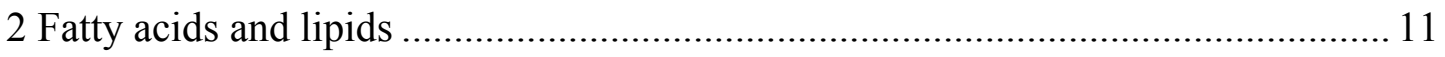

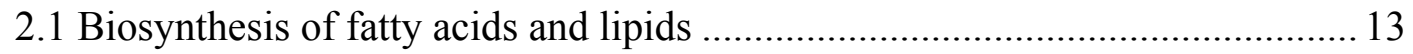

2.2 Biosynthesis of polyunsaturated fatty acids (PUFAs) ................................. 15

2.3 Engineering of VLCPUFA synthesis in plants ................................................. 18

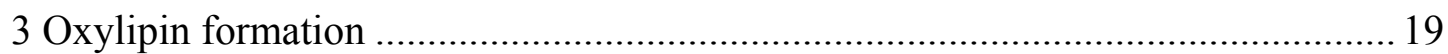

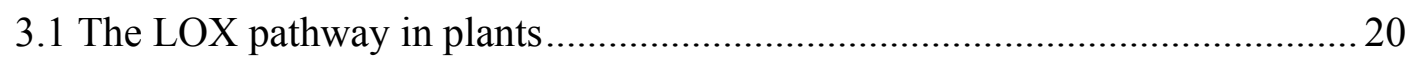

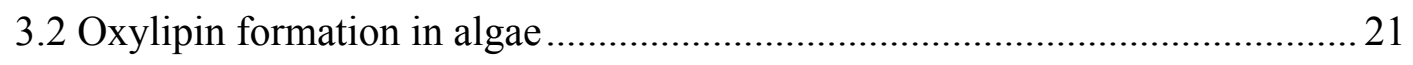

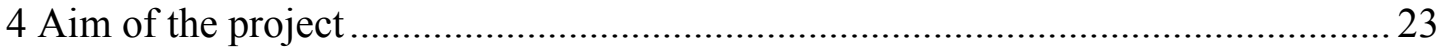

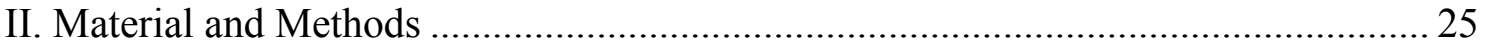

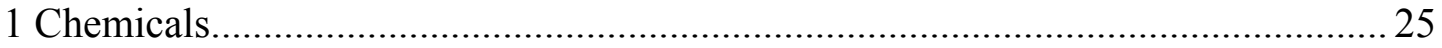

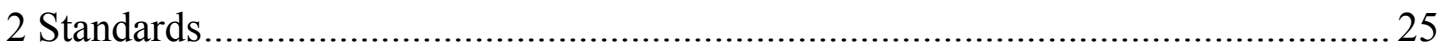

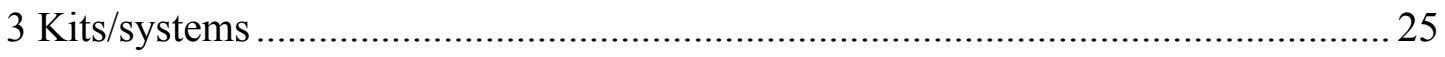

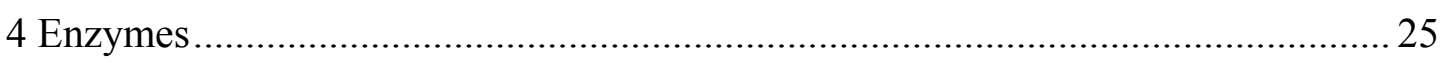

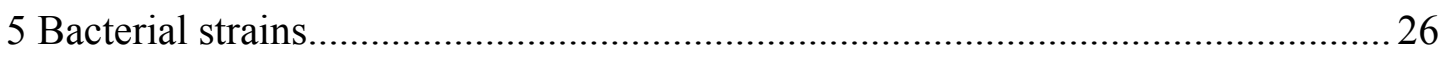

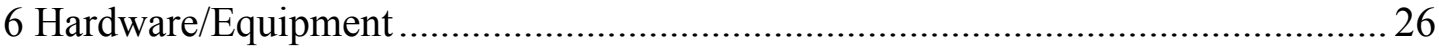

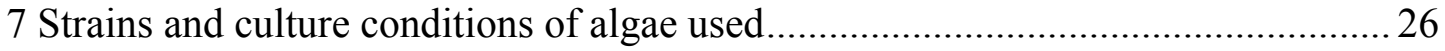

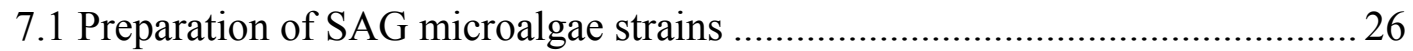

7.2 Cultivation of Prymnesium parvum SAG 127.79 and P. saltans SAG 18.97 .. 27

7.3 Cultivation of Nostoc punctiforme PCC 73102 and Nostoc sp. SAG 25.82 .... 27

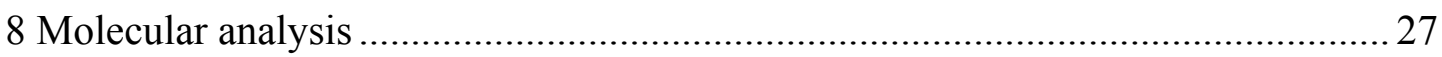

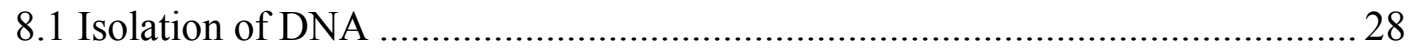

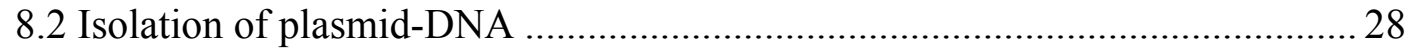

8.3 Isolation of $N p L O X 1$ and $N p L O X 2$ from $N$. punctiforme PCC $73120 \ldots \ldots \ldots \ldots . . .28$

8.4 Isolation of Peroxidase-LOX fusion protein from Nostoc sp. SAG 25.82 ....... 29

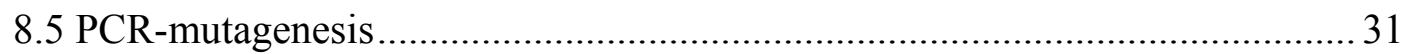

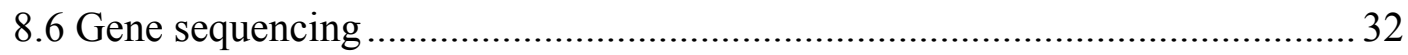




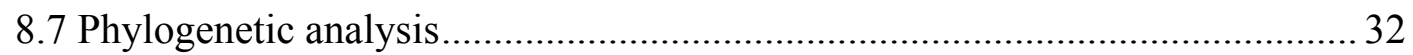

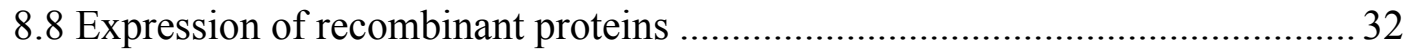

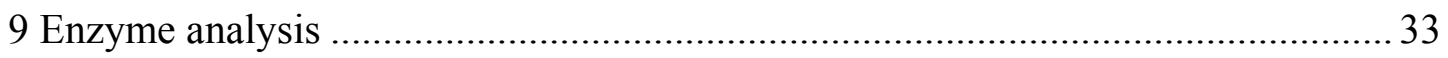

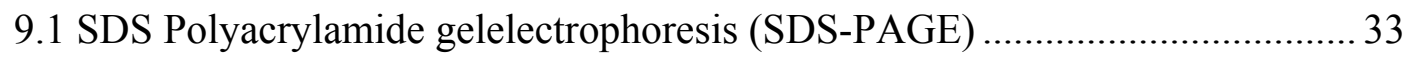

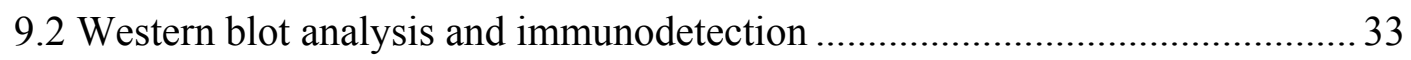

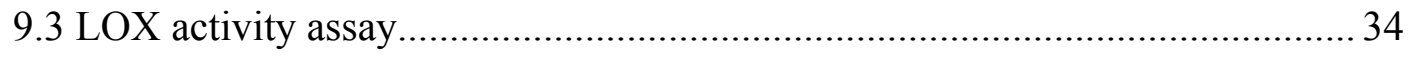

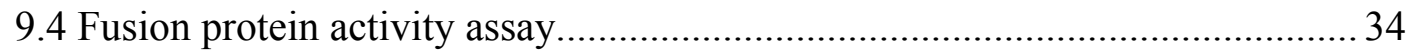

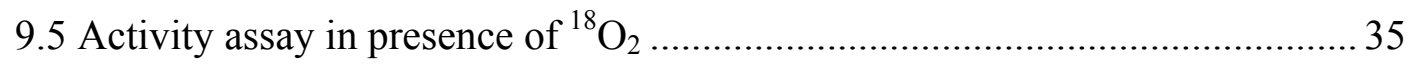

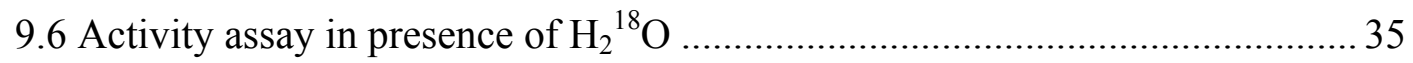

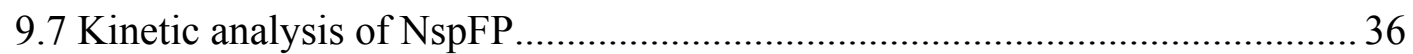

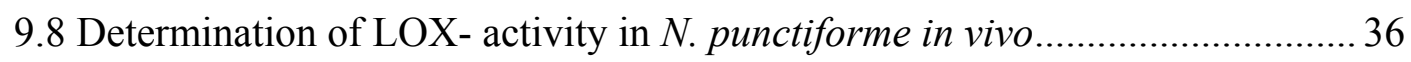

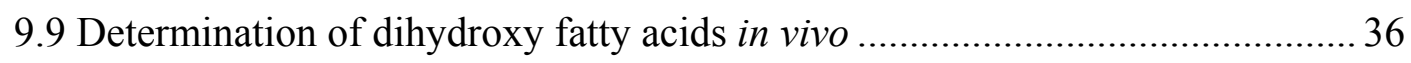

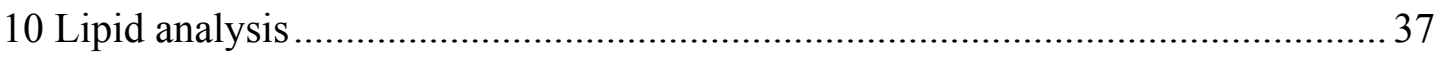

10.1 Lipid extraction and thin layer chromatography (TLC) …......................... 37

10.2 Alkaline hydrolysis, transesterification and extraction of FAMEs................ 37

10.3 Acidic hydrolysis, methylation of FA carboxyl groups and extraction of

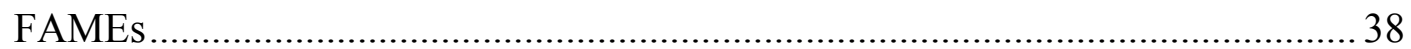

10.4 Preparation of 4,4-dimethyloxaline (DMOX) derivatives .............................. 38

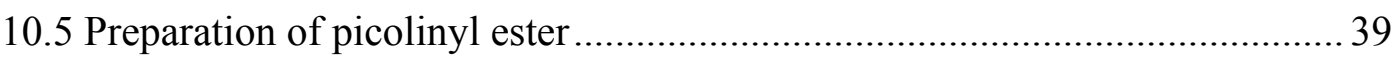

10.6 Identification of FAMEs by gas chromatography (GC) with flame ionisation

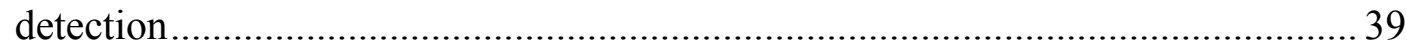

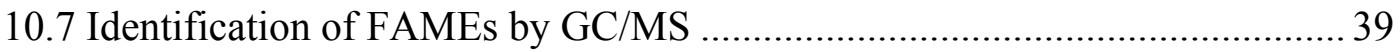

10.8 Identification and preparation of FAs and oxylipins by high performance

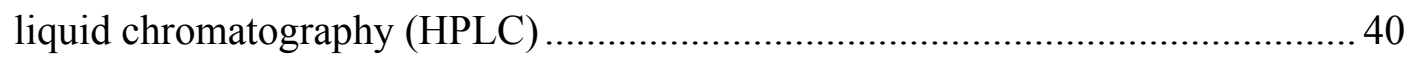

10.9 Analysis of NspFP products by HPLC/MS analysis ................................... 41

10.10 Nucleic magnet resonance (NMR) analysis............................................ 41

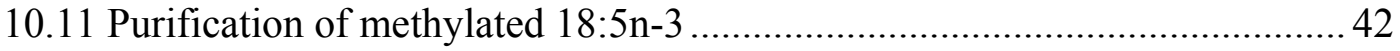

10.12 Oxylipin profiling of SAG microalgal strains …....................................... 42

10.13 Oxylipin profiling of the cyanobacteria Nostoc punctiforme and Nostoc sp.43

10.14 Feeding experiment with labelled fatty acid substrates ............................... 44

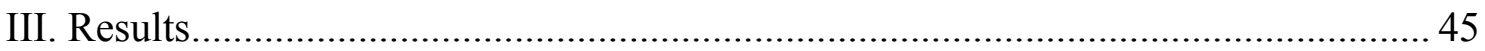

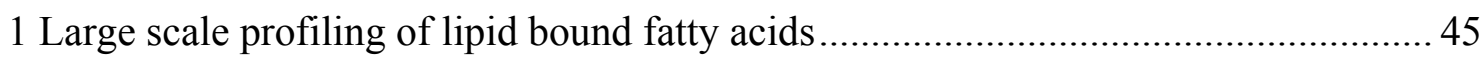

1.1 A database of profiles for diverse microalgae ................................................ 45

1.2 Occurrence of PUFAs within different taxonomic groups ................................ 51 


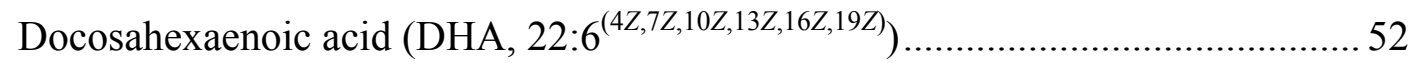

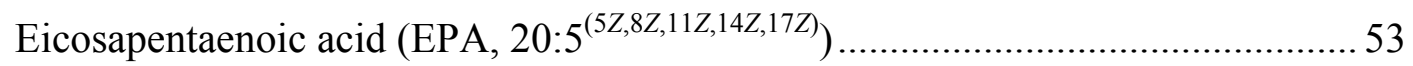

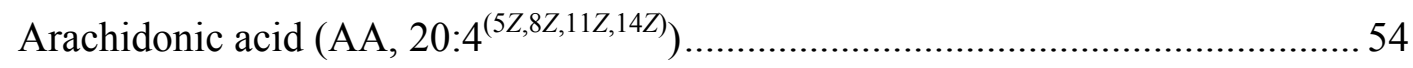

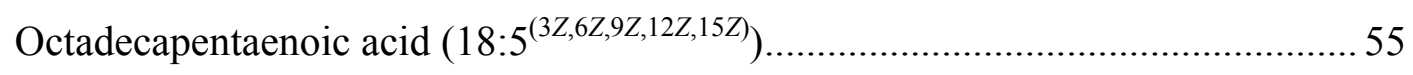

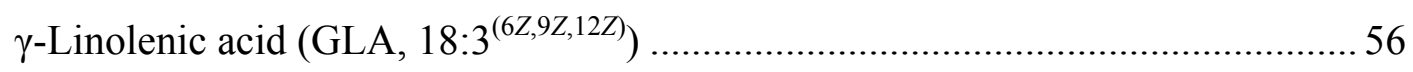

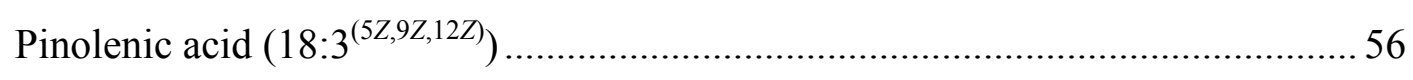

Hexadecatetraenoic acid $\left(16: 4^{(4 Z, 7 Z, 10 Z, 13 Z)}\right)$....................................................... 57

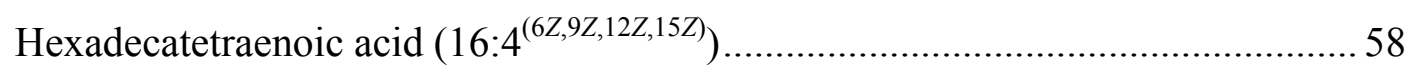

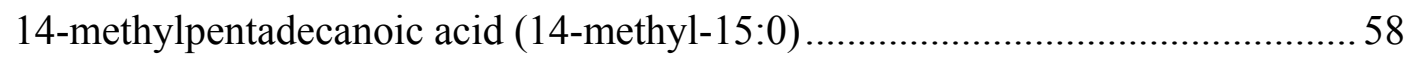

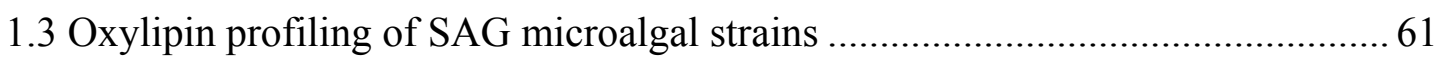

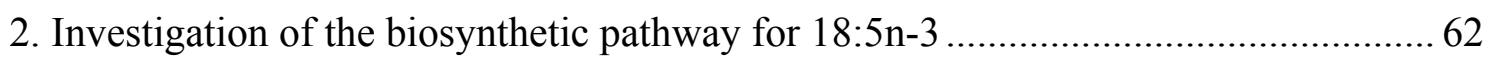

2.1 Selection of algae strains suitable for 18:5n-3 isolation .................................... 62

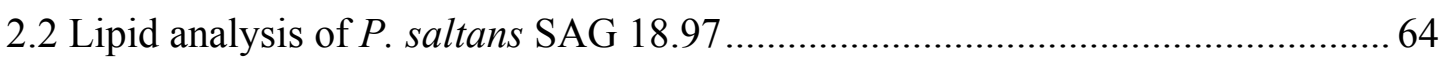

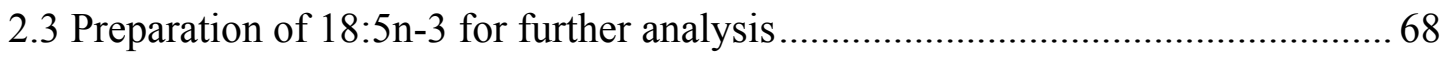

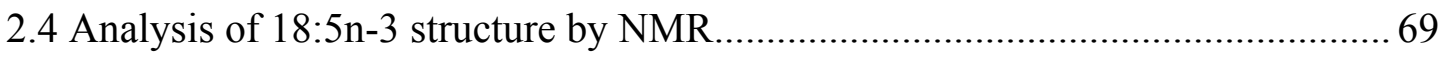

2.5 Biosynthesis of 18:5n-3 in P. parvum SAG 127.79 ......................................... 70

3. Lipoxygenase pathway of selected cyanobacterial strains ...................................... 76

3.1 Phylogenetic analysis of new lipoxygenases from cyanobacteria ........................ 76

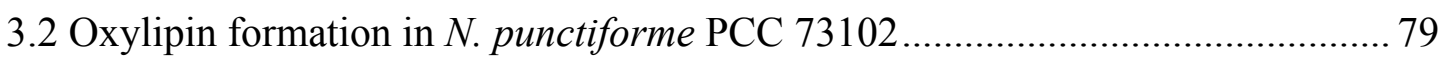

3.2.1 Amino acid sequence alignment with other LOX sequences ....................... 79

3.2.2 Characterisation of in vitro LOX activity of NpLOX1 and NpLOX2 .......... 81

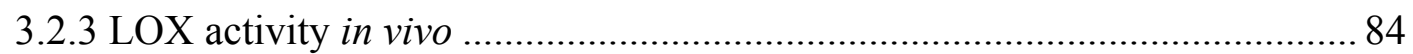

3.3 Characterisation of a peroxidase-LOX fusion protein from Nostoc sp. ............... 87

3.3.1 Alignment of individual peroxidase and LOX domains of the fusion protein

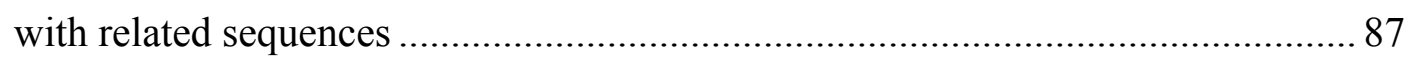

3.3.2 The LOX domain of Nostoc sp is a linoleate (9R)-LOX ............................ 90

3.3.3 The peroxidase domain is enzymatically not active with the reaction products

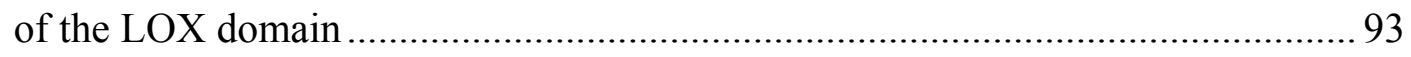

3.3.4 Production of dihydroxy fatty acids by the NspFP ...................................... 94

3.3.5 Comparison of endogenous oxylipins in different cyanobacterial strains ... 101

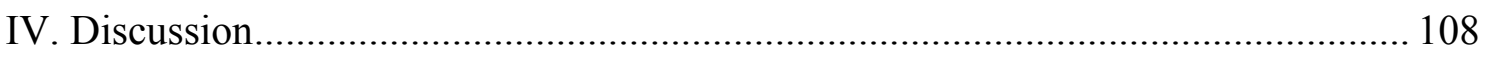

1. Microalgae as sources of PUFAs and genes for plant engineering .................... 108

2. Fatty acid profile as chemotaxonomical marker for microalgae ......................... 113 
3. Analysis of biosynthesis of 18:5n-3 in P. parvum as a prerequisite for gene isolation

4. LOX pathway of two Nostoc species

4.1 Two LOXs from $N$. punctiforme with interesting characteristics................... 126

4.2 The peroxidase-LOX fusion protein from Nostoc sp. SAG 25.82 ................. 130

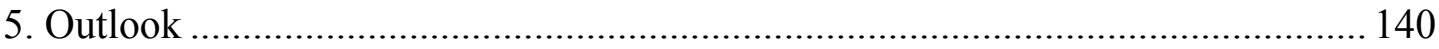

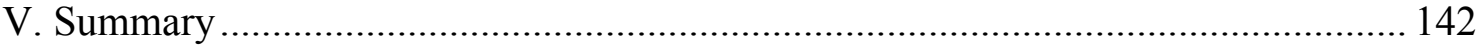

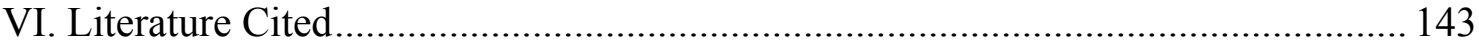

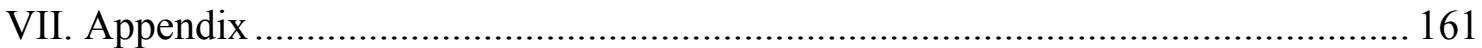

1. Dihydroxy FA products of conversion of LA, ALA, GLA and AA by NspFP 161

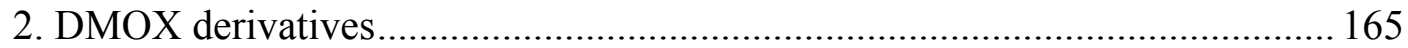




\section{Figures}

Figure 1 Schematic representation of VLCPUFA biosynthesis via the $\omega 6$ and $\omega 3$ pathway.

Figure 2 Schematic overview of the plant LOX pathway.

Figure 3 Diversity of taxonomic groups of microalgae maintained in the SAG

Figure 4 Scheme of the peroxidase-LOX fusion protein gene and the 29 primers used for the amplification of three fragments.

Figure 5 Scheme of the PCR mutagenesis.

Figure 6 GC chromatogram of Chlamydomonas sp. and the standard F.A.M.E. Mix".

Figure 7 Mass spectrum of the DMOX derivative of 16:3(7Z,10Z,13Z)

Figure 8 Example of data compiled in the FAME database established of all SAG microalgae strains screened.

Figure 9 FAME profiles of different algal genera.

Figure 10 Overview of DHA and EPA distribution in different taxonomic groups of microalgae.

Figure 11 Overview of AA, GLA, 18:5n-3, pinolenic acid, 16:4n-3 and 14 methyl-15:0 distribution in different taxonomic groups of microalgae.

Figure 12 FAME profile of $P$. parvum and P. saltans.

Figure 13 Growth curve of $P$. saltans and P. parvum.

Figure 14 Analysis of lipid classes isolated from $P$. saltans by TLC.

Figure 15 GC/MS analysis of 18:5n-3 isolated via RP-HPLC.

Figure 16 RP-HPLC analysis of feeding experiments with $\left[1-{ }^{14} \mathrm{C}\right]-\mathrm{LA}$.

Figure 17 Relative proportion of FAMEs within three $P$. parvum SAG

127.79 cultures grown with and without labelled FAs.

Figure 18 GC/MS analysis of FAMEs of $P$. parvum supplemented with labelled FAs.

Figure 19 Comparative phylogenetic analysis of NpLOX1, NpLOX2 and NspFP. 
Figure 20 Partially deduced amino acid sequence alignment of NpLOX1 and 80 NpLOX2 with other LOX sequences.

Figure 21 HPLC analysis of products formed by NpLOX1 and NpLOX2 with LA.

Figure 22 Production of 13-HODE and regiospecificity of NpLOX1 in dependence of different $\mathrm{pH}$-values.

Figure 23 SDS-PAGE of NpLOX2 in pEXP5-NT expressed in different $E$. coli expression strains.

Figure 24 Formation of oxylipins upon wounding of $N$. punctiforme.

Figure 25 Partially deduced amino acid sequences alignment of peroxidase and LOX domain of $N$. sp.. SAG 25.82 fusion protein.

Figure 26 HPLC analysis of reaction products of the LOX domain.

Figure 27 pH-optimum of NspFP with ALA.

Figure 28 Substrate preference of LOX domain.

Figure 29 Western blot analysis of the peroxidase domain expressed in $E$. coli BL21 expression cells.

Figure 30 Separation of the products of LA conversion catalysed by the NspFP using RP-HPLC.

Figure 31 GC/MS analysis of 9,14-diHODE converted by NspFP.

Figure 32 Kinetic analysis of LA conversion catalysed by NspFP.

Figure 33 Free oxylipin profiles of different cyanobacterial strains.

Figure 34 Esterified oxylipin profiles of different cyanobacterial strains.

Figure 35 Illustration of evolutionary relationship and divergence times for red, green, glaucophyte and chromist (chlorophyll-c-containing cryptophytes, stramenopiles, haptophytes) algae.

Figure 36 Microalgal classes producing EPA in higher proportions than $10 \%$ of total FAs.

Figure 37 Microalgal classes producing 18:5n-3 in proportions $>10 \%$ of total FAs.

Figure 38 Comparing structures of betaine ether-linked lipids and PC.

Figure 39 General scheme of the LOX reaction with LA as substrate.

Figure 40 Models explaining the positional specificity of LOXs. as substrates. 
Figure 42 Mechanism proposed for the peroxidase domain of NspFP.

Figure 43 Mass spectrum of 9,10-diHODE.

Figure 44 Mass spectrum of 8,11-diHODE.

Figure 45 Mass spectrum of 9,16-diHOTE.

Figure 46 Mass spectrum of 9,13-diHOTE.

Figure 47 Mass spectrum of 9,14-diHOTE.

Figure 48 Mass spectrum of 11,16-diHETE.

Figure 49 Mass spectrum of the DMOX derivative of 16:1(6Z).

Figure 50 Mass spectrum of the DMOX derivative of 16:1(7Z).

Figure 51 Mass spectrum of the DMOX derivative of 16:1(8Z).

Figure 52 Mass spectrum of the DMOX derivative of 16:1(11Z).

$\begin{array}{ll}\text { Figure } 53 \text { Mass spectrum of the DMOX derivative of 16:2(7Z,10Z). } & 167\end{array}$

Figure 54 Mass spectrum of the DMOX derivative of 16:3(4Z,7Z,10Z). 168

$\begin{array}{lll}\text { Figure } 55 \text { Mass spectrum of the DMOX derivative of 17:1(9Z). } 168 & 160\end{array}$

Figure 56 Mass spectrum of the DMOX derivative of 18:2(9Z,14Z). 169

$\begin{array}{lll}\text { Figure } 57 \text { Mass spectrum of the DMOX derivative of 18:3(5Z,9Z,12Z). } & 169\end{array}$

Figure 58 Mass spectrum of the DMOX derivative of 18:4(5Z,9Z,12Z,15Z). 170

Figure 59 Mass spectrum of the DMOX derivative of 19:1(12Z). 170

\section{Tables}

Table 1 Overview of FAMEs and other substances identified in the microalga strains analysed

Table2 Substances identified with GC/MS analysis.

Table 3 Ten algae containing highest proportions of DHA. 52

Table $4 \quad$ Ten algae containing highest proportions of EPA. 53

Table 5 Ten algae containing highest proportions of AA. 55

Table 6 Ten algae containing highest proportions of 18:5n-3. 55

Table 7 Ten algae containing highest proportions of GLA. 56

Table $8 \quad$ Ten algae containing highest proportions of $18: 3(5 Z, 9 Z, 12 Z) . \quad 57$

Table 9 Ten algae containing highest proportions of 16:4n-3. 57 
Figures and Tables

Table $10 \quad$ Algae containing highest proportions of 16:4n-1.

58

Table 11 Ten algae containing the highest proportions of 14-methyl-15:0

59

Table 12 FAME composition of purified neutral-, glycol-, phospho- and betaine lipids of $P$. saltans.

Table $13 \quad$ NMR data of 18:5n-3. 70

Table $14 \quad$ FA hydroperoxides formed by NpLOX1 in vitro with different 82 FA substrates

Table 15 Selected NMR data of 9,14-diHODE (solvent $\mathrm{CDCl}_{3}$ ). 96

Table 16 Overview of the dihydroxy FAs formed in the reaction of 97 NspFP with different FA substrates. 


\section{Abbreviations}

\begin{tabular}{|c|c|}
\hline${ }^{\circ} \mathrm{C}$ & degree Celsius \\
\hline$(9 R)-\mathrm{H}(\mathrm{P}) \mathrm{ODE}$ & $(9 R, 10 E, 12 Z)-9$-hydro(pero)xy-10,12-octadecadienoic acid \\
\hline$(9 R)-\mathrm{H}(\mathrm{P}) \mathrm{OTE}$ & $\begin{array}{l}(9 R, 10 E, 12 Z, 15 Z) \text {-9-hydro(pero)xy-10,12,15-octadecadienoic } \\
\text { acid }\end{array}$ \\
\hline$(11 R)-\mathrm{HETE}$ & $(5 Z, 8 Z, 11 R, 12 E, 14 Z)$-11-hydroxy-5,8,12,14-eicosatetraenoic acid \\
\hline$(12 R, 13 S)$-diHETE & $\begin{array}{l}(5 Z, 8 Z, 10 E, 14 Z)-12 R, 13 S \text {-dihydroxy-5,8,10,14-eicosatetraenoic } \\
\text { acid }\end{array}$ \\
\hline (12S)-HPETE & $\begin{array}{l}(5 Z, 8 Z, 10 E, 12 S, 14 Z)-12 S \text {-hydroperoxy-5,8,10,14-eicosatetraenoic } \\
\text { acid }\end{array}$ \\
\hline $13 \gamma-\mathrm{H}(\mathrm{P}) \mathrm{OTE}$ & $(6 Z, 9 Z, 11 E, 13 S)$-13-hydro(pero)xy-6,9,11-octadecatrienoic acid \\
\hline$(13 S)-\mathrm{H}(\mathrm{P}) \mathrm{ODE}$ & $(9 Z, 11 E, 13 S)$-13-hydro(pero)xy-9,11-octadecadienoic acid \\
\hline$(13 S)-\mathrm{H}(\mathrm{P}) \mathrm{OTE}$ & $\begin{array}{l}(9 Z, 11 E, 13 S, 15 Z)-13 \text {-hydro(pero)xy-9,11,15- } \\
\text { octadecatrienoic acid }\end{array}$ \\
\hline$(15 S)-\mathrm{H}(\mathrm{P}) \mathrm{ETE}$ & $\begin{array}{l}(5 Z, 8 Z, 11 Z, 13 E, 15 S)-15 \text {-hydro(pero)xy-5,8,11,13- } \\
\text { eicosatetraenoic acid }\end{array}$ \\
\hline ANS & 8-anilino-1-naphthalene-sulfonic acid \\
\hline $\mathrm{AA}$ & arachidonic acid \\
\hline $\mathrm{ACP}$ & acyl carrier protein \\
\hline ADP & adenosine diphosphate \\
\hline ALA & $\alpha$-linolenic acid \\
\hline amu & atomic mass unit \\
\hline $\mathrm{AOC}$ & allene oxide cyclase \\
\hline AOS & allene oxide synthase \\
\hline At & Arabidopsis thaliana \\
\hline ATP & adenosine triphosphate \\
\hline $\mathrm{bp}$ & base pair(s) \\
\hline cDNA(s) & copy-desoxyribonucleic acid(s) \\
\hline CDP-DAG & cytidine diphosphodiacylglycerol \\
\hline $\mathrm{CoA}$ & Coenzyme A \\
\hline CP-HPLC & chiral phase high performance liquid chromatography \\
\hline $\mathrm{d}$ & $\operatorname{day}(\mathrm{s})$ \\
\hline DAG & diacylglycerol \\
\hline DAGAT & diacylglycerol-acyltransferase \\
\hline $\mathrm{ddH}_{2} \mathrm{O}$ & double distilled water \\
\hline DES & divinyl ether synthase \\
\hline $\operatorname{DGD}(\mathrm{G})$ & digalactosyldiacylglycerol \\
\hline DGCC & 1,2-diacylglyceryl-3-O-carboxy-(hydroxymethyl)-choline \\
\hline DGTA & $\begin{array}{l}\text { 1,2-diacylglyceryl-3-O-2'-(hydroxymethyl)-( } N, N, N \text {,-trimethyl)- } \\
\beta \text {-alanine }\end{array}$ \\
\hline DGTS & 1,2-diacylglyceryl-3-O-4'-( $N, N, N$,-trimethyl)-homoserine \\
\hline DHA & docosahexaenoic acid \\
\hline DMOX & 4,4-dimethyloxaline \\
\hline DNA & desoxyribonucleic acid \\
\hline d.w. & dry weight \\
\hline EAS & epoxy alcohol synthase \\
\hline e.g. & exempli gratia \\
\hline EPA & eicosapentaenoic acid \\
\hline
\end{tabular}




\begin{tabular}{|c|c|}
\hline ER & endoplasmatic reticulum \\
\hline et al. & et altera \\
\hline EtOH & ethanol \\
\hline $\mathrm{eV}$ & electron volt(s) \\
\hline $\mathrm{FA}(\mathrm{s})$ & fatty $\operatorname{acid}(\mathrm{s})$ \\
\hline FAME(s) & fatty acid methyl ester(s) \\
\hline FAS & fatty acid synthase \\
\hline fmol & femtomol \\
\hline Fig. & Figure \\
\hline f.w. & fresh weight \\
\hline g & gravity \\
\hline G3P & glycerinaldehyde-3-phosphate \\
\hline GBq & gigabequerel \\
\hline $\mathrm{GC}$ & gas chromatography \\
\hline GLA & $\gamma$-linolenic acid \\
\hline $\mathrm{Gm}$ & Glycine max \\
\hline $\mathrm{h}$ & hour(s) \\
\hline HPL & hydroperoxide lyase \\
\hline HPLC & high performance liquid chromatography \\
\hline IS & internal standard \\
\hline JA & jasmonic acid \\
\hline $\mathrm{k}$ & kilo \\
\hline K & Kelvin \\
\hline $\mathrm{kV}$ & kilovolt(s) \\
\hline 1 & litre(s) \\
\hline LA & linoleic acid \\
\hline Lc & Lens culinaris \\
\hline LDS & linoleate diol synthase \\
\hline Le & Lycopersicon esculentum \\
\hline $\mathrm{LOX}(\mathrm{s})$ & lipoxygenase(s) \\
\hline M & molar \\
\hline $\mathrm{mA}$ & milliampere(s) \\
\hline $\mathrm{Mbq}$ & megabequerel \\
\hline $\mathrm{mm}$ & millimetre(s) \\
\hline $\mathrm{mM}$ & millimolar \\
\hline mmol & millimol \\
\hline meLA & methylated linoleic acid \\
\hline $\mathrm{mg}$ & milligram(s) \\
\hline $\operatorname{MGD}(\mathrm{G})$ & monogalactosyldiacylglycerol \\
\hline Mhz & megaherz \\
\hline $\min$ & minute(s) \\
\hline $\mathrm{ml}$ & millilitre(s) \\
\hline MS & mass spectrometry \\
\hline $\mathrm{mt}$ & mutant \\
\hline$\mu g$ & $\operatorname{microgram}(\mathrm{s})$ \\
\hline$\mu 1$ & microlitre(s) \\
\hline$\mu \mathrm{m}$ & micrometer(s) \\
\hline$\mu \mathrm{M}$ & micromolar \\
\hline$\mu \mathrm{mol}$ & micromol \\
\hline $\mathrm{nM}$ & nanomolar \\
\hline nmol & nanomol \\
\hline
\end{tabular}




\begin{tabular}{|c|c|}
\hline $\mathrm{Np}$ & Nostoc punctiforme \\
\hline NspFP & Peroxidase-LOX fusion protein from Nostoc sp. \\
\hline $\mathrm{Nt}$ & Nicotiana tabacum \\
\hline NMR & nucleic magnet resonance \\
\hline OD & optical density \\
\hline oPDA & $(9 S, 13 S)$-12-oxo phyto-9,13-dienoic acid \\
\hline $\mathrm{P}$ & pellet \\
\hline PA & phosphatidic acid \\
\hline PAGE & polyacrylamide gelelectrophoresis \\
\hline $\mathrm{PC}$ & phosphatidylcholine \\
\hline PCC & Pasteur culture collection \\
\hline PCR & polymerase chain reaction \\
\hline PDAT & phospholipid diacylglycerol acyltransferase \\
\hline PE & phosphatidylethanolamine \\
\hline PG & phosphatidylglycerol \\
\hline $\mathrm{PGG}_{2}$ & hydroperoxy-endoperoxide prostaglandin $\mathrm{H}_{2}$ \\
\hline PGH & prostaglandin $\mathrm{H}$ \\
\hline PI & phosphatidylinositol \\
\hline PKS & polyketidesynthase \\
\hline pmol & picomol \\
\hline POX & peroxygenase \\
\hline $\mathrm{Pp}$ & Physcomitrella patens \\
\hline ppm & parts per million \\
\hline Ppu & Porphyra purpureum \\
\hline Ps & Pisum sativum \\
\hline PS & phosphatidylserine \\
\hline (vlc) PUFA(s) & (very long chain) polyunsaturated fatty acid(s) \\
\hline RNA & ribonucleic acid \\
\hline rpm & rounds per minute \\
\hline RP-HPLC & reverse phase high performance liquid chromatography \\
\hline RT & room temperature \\
\hline SAG & culture collection of microalgae in Göttingen \\
\hline SDS & sodium dodecyl sulphate \\
\hline $\mathrm{s}$ & second(s) \\
\hline $\mathrm{SE}(\mathrm{s})$ & steryl ester(s) \\
\hline $\mathrm{SN}$ & supernatant \\
\hline SP-HPLC & Straight phase high performance liquid chromatography \\
\hline $\mathrm{SQD}(\mathrm{G})$ & sulfoquinovosyldiacylglycerol \\
\hline $\mathrm{St}$ & Solanum tuberosum \\
\hline Tab. & table \\
\hline TAG(s) & triacylglycerol(s) \\
\hline TLC & thin layer chromatography \\
\hline USA & United States of America \\
\hline UV & ultraviolet \\
\hline $\mathrm{v}$ & volume \\
\hline V & $\operatorname{volt}(s)$ \\
\hline $\mathrm{W}$ & weight \\
\hline $\mathrm{WE}(\mathrm{s})$ & wax ester(s) \\
\hline wt & wildtype \\
\hline
\end{tabular}




\section{Introduction}

The accessibility of the ocean as a source for new organic compounds is closely connected with the research on the marine microbial diversity. Therefore knowledge on diversity and physiology of marine micro-organisms is increasing and paralleled by an upcoming interest to investigate their biochemistry. Even the high diversity of algae suggests a multiple number of interesting metabolites like oils/fats, proteins and carbohydrates, which may be important for the chemical industry, human diet and medical applications.

At present oils and fats are the major raw materials being used by the chemical industry from renewable resources (Drexler et al. 2003). Particular lipids play a role as pigments, cofactors, detergents, transporters (dolicholes), and hormones (vitamin D-derivates, sexual hormones). The latter may serve as intra- and intercellular signal molecules like eicosanoids and octadecanoids (Gerwick 1999b). The range of applications of animal and plant oils is almost as various as that of oil produced by petrochemistry. Plant oils, which cannot be produced by petrochemistry, are therefore most interesting for commercial purposes. The diversity of chain length, number and position of double bonds as well as functional groups of fatty acids (FAs) of glycerolipids leads to a broad range of different and valuable oils (e.g. high oleic acid-oils). Most likely many of these oils have not been discovered yet and might be suitable for the development of new products. For exploration and optimal utilization of the diversity of FAs, proceedings in the field of algae are expected.

\section{Microalgae as living cell factories}

Microalgae are an enormously diverse group of primary producers, abundant in almost all ecosystems on earth ranging from marine and freshwater environments to desert sands, from hot springs to snow and ice. More than half of the total primary production at the base of the food chain worldwide is accounted to microalgae (Guschina and Harwood 2006b) which have key roles in carbon fixation and oxygen production. Due to their high physiological diversity microalgae have long been proposed as living "cell factories", as they produce an enormous variety of high value compounds for chemical industry, human diet and medicine, such as provitamin A carotenoids and polyketides, including antibiotics (Cardozo et al. 2006) . Taking into account that bio products are 
gaining interest, algae are one natural source providing novel biologically active substances (Tringali 1997; Burja et al. 2001; Mayer and Hamann 2004; Blunt et al. 2005; Mayer and Hamann 2005; Singh et al. 2005). Even though there has been considerable interest in the field in recent years, only a limited number of microalgae have been exploited for biotechnological applications so far. In contrast to microalgae, the food industry of many countries uses macroalgae as a source of fibres, minerals, vitamins and different antioxidants. In the recent years, the emphasis has moved from wild harvests to farming and controlled cultivation to produce valuable new products on a large scale (Cardozo et al. 2006). One important advance in this field is the development of large photo bioreactors, which facilitate the cultivation of microalgae. An example is the production of the red pigment astaxanthin, which is synthesised by the green algae Haematococcus (Johnson and An 1979). Astaxanthin is used to colour marine invertebrates like lobsters, crabs and shrimps and also to give salmon and trout the distinctive orange red colour (Johnson and An 1979).

\subsection{Microalgae as a source of polyunsaturated fatty acids (PUFAs)}

As microalgae are the primary producers of the food chain, they provide a wide range of different PUFAs which are essential to organisms in the upper food chain. In pharmacology, medicine and food science PUFAs play a major role among the FAs found in lipids. They are essential for normal cell function and play important roles in obesity and cardiovascular diseases (Gill and Valivety 1997; Napier and Sayanova 2005). PUFAs are mostly esterified and occur within the group of glycerolipids. Their fundamental meaning for mammals lies in the presence of double bonds beyond position C9. Mammals are dependent on these PUFAs since they cannot introduce the $\Delta^{12}$ - and $\Delta^{15}$ - double bonds that are needed for the synthesis of linoleic acid (LA) and $\alpha$ linolenic acid (ALA) due to a lack of the regarding enzymes. Moreover LA and ALA are essential FAs because they serve as important precursors for the synthesis of further longer and higher unsaturated PUFAs, the very long chain PUFAs (VLCPUFAs). These are eicosapentaenoic acid (EPA) and docosahexaenoic acid (DHA) for example, which are known to be cardiovascular-protective components of the human diet (Hites et al. 2004). Therefore food should contain these essential FAs, which is given by serving fish and vegetables several times per week. Fish do not synthesise the VLCPUFAs de novo, 
but these compounds derive from marine micro-organisms they consume (Cardozo et al. 2006). Moreover the fish oil quality depends on fish species and globally available fish resources which are declining as well as the fact that fish accumulates poison over the food chain, requires new sources for VLCPUFAs. Thus, microalgae producing all sorts of different PUFAs in high amounts might represent an acceptable alternative.

In particular there is an increasing interest in EPA, which is found in a wide variety of marine microalgal classes. But only a few microalgal species show potential for industrial production, e.g. the red alga Porphyridium cruentum and the eustigmatophyte Nannochloropsis oculata (Cohen 1999; Sukenik 1999). In addition, an efficient large scale cultivation system and purification process is required to produce microalgal EPA for commercial purposes (Lebeau and Robert 2003). Many detailed investigations on growth behaviour and PUFA production in several suitable microalgae were performed (Guschina and Harwood 2006a) indicating, that PUFA production and accumulation in microalgae are complex procedures and difficult to optimise. However, PUFA production has mainly been applied in the aquaculture industry to enrich fish oil for human diet.

\subsection{Screening for new FAs and lipids from microalgae}

The analysis of the profiles of FAs as well as their occurrence in different lipid classes in microalgae is an emerging field which is expected to achieve the identification of novel FAs with a variety of functional groups (Bergé and Barnathan 2005). Taken together many investigations have been carried out and published, describing the contents as well as the composition of PUFAs in mostly marine microalgae (Viso and Marty 1993; Dunstan et al. 1994; Tonon et al. 2002), though systematic approaches including different or even many genera are still missing. Beside the identification of novel FAs, some recent studies report on the use of FA and lipid profiles of algae as biomarkers (Volkman et al. 1998; Bergé and Barnathan 2005; Schweder et al. 2005; Rossi et al. 2006; Temina et al. 2006). Viso et al. (1993) determined profiles of FAs of nine different marine algae groups and they were able to define species dependent lipid compositions. Moreover they found a roughly taxon specific profile, when the cells were cultured under identical growth conditions. Recently, Temina et al. (2006) screened for FAs of several species of the cyanobacterium Nostoc and described the application of cluster analysis for the identification of FAs from different members of genus Nostoc. Further they discussed the necessity of studying the profiles of as many 
strains as possible. The data currently available splits freshwater microalgae into two groups, concerning their profile of FAs. On one hand those cyanobacteria and Chlorophyta that contain a low amount of FAs, predominantly saturated and single unsaturated FAs as well as trace amounts of PUFA (mostly LA). On the other hand the second group comprising Chrysophyceae, Haptophyceae, Cryptophyceae and Dinophyceae contains significant amounts of PUFAs (Watson 2003).

\section{Fatty acids and lipids}

Plants and algae share a fundamental biochemical process, which is photosynthesis. Therefore the overall cellular organisation is very similar in many respects. According to the endosymbiosis theory plastids originated as separate prokaryotic algae which were taken inside as cell as endosymbionts (Gray 1992; Yoon et al. 2004; Batthacharya and Medlin 2005). Due to the assumption that higher plants developed from green algae (Cavalier-Smith 1986), the FA and lipid metabolism is explained with respect to existing data from plants and not from animals.

Lipids comprise a large group of FA derived compounds as well as many pigments and secondary compounds which are not related to the FA metabolism (Somerville et al. 2000). In plants, lipids fulfil a wide range of functions, such as major components of biological membranes, chemical reserve of free energy and as waxes to coat and protect the plant from the environment. Furthermore they play important roles in signal transduction, e.g. as substrates for the synthesis of the growth regulator jasmonic acid (JA). The most common lipids in plants, the glycerolipids, consist of two FAs esterified to the $\mathrm{C} 1(s n-1)$ and $\mathrm{C} 2(s n-2)$ of a glycerol backbone, whereas to the third position (sn3) moieties of different chemical properties are bound. Due to their cellular function lipids can be subdivided into two main groups: (1) the storage lipids and (2) structural or membrane lipids. Storage lipids, also called neutral lipids, lack by definition charged groups and are therefore unable to integrate into bilayer membranes (Athenstaedt and Daum 2006). The group of neutral lipids is formed by triacylglycerols (TAGs), steryl esters (SEs) and wax esters (WEs). Due to their head groups the structural lipids can further be subdivided into phospholipids, glycolipids and sphingolipids. Phospholipids consist of two FAs bound to the $s n-1$ and $s n-2$ position of the glycerol backbone and a phosphate group at the $s n-3$ position, forming phosphatidic acid (PA). All other 
phospholipids derive from PA by esterification of different nitrogen containing alcohols or organic compounds like choline to the phosphate group. These phospholipids are phosphatidylcholine (PC), phosphatidylethanolamine (PE), phosphatidylglycerol (PG) and phosphatidylserine (PS). In addition a unique phospholipid with a dimeric structure is found exclusively in bacteria and mitochondria. The trivial name of this phospholipid is cardiolipin (CL), but correctly termed 1,3-bis(sn-3-phosphatidyl)-sn-glycerol. Generally CL is assumed to provide stability to respiratory chain super complexes (Gohil et al. 2004) and was found to be an integral constituent of the bacterial photoreaction centre (McAuley et al. 1999) and photosystem II complex in higher plants.

In contrast, the head group of glycolipids consist of mono- or polysaccharides, which are covalently bound to the $s n-3$ positions. The glycolipids mono- and digalactosyldiacylglycerol as well as sulfoquinovosyldiacylglycerol (MGD, DGD and SQD) constitute the bulk of lipids in the photosynthetic membranes of the plastids (Browse and Somerville 1994). Finally, sphingolipids comprise a complex range of lipids in which FAs are linked via amide bonds to a long-chain amino alcohol. The basic sphingolipids is ceramide, which can be modified to more complex sphingolipids such as glucosylsphingolipids (Somerville et al. 2000).

In addition some algae, fungi and mosses harbour another class of lipids: the betaine lipids. These glycerolipids contain a betaine linked by an ether bond at the $s n-3$ position of the glycerol moiety, besides the FAs esterified in the $s n-1$ and $s n-2$ positions. Three different types of betaine lipids are known: 1,2-diacylglyceryl-3-O-4'-( N,N,Ntrimethyl)-homoserine (DGTS), 1,2-diacylglyceryl-3-O-2'-(hydroxymethyl)-( $N, N, N$ trimethyl)- $\beta$-alanine (DGTA) and 1,2-diacylglyceryl-3-O-carboxy-(hydroxymethyl)choline (DGCC). Many DGTS producing organisms contain only low or negligible levels of the phospholipid phosphatidylcholine (PC), which lead to the suggestion that these two lipid classes are interchangeable. This hypothesis is supported by the high similarity of the structure of betaine lipids and PC (Rozentsvet et al. 2000). In addition it was found, that the photobacterium Rhodobacter sphaeroides accumulated betaine lipids when the bacteria where grown under phosphate-limiting conditions (Benning et al. 1995). 


\subsection{Biosynthesis of fatty acids and lipids}

The primary FA biosynthesis in plants takes place in the plastid, whereas in vertebrates it takes place in the cytoplasm (Ohlrogge and Kuo 1985). The responsible enzyme is an enzyme complex, named fatty acid synthase (FAS). The initial substrate malonyl-CoA is formed in a two step reaction by acetyl-CoA carboxylase (ACCase). In plants two different isoforms of ACCase exist. One is localised in the plastid und consists of four subunits whereas the cytosolic form is a large homodimeric protein (Somerville et al. 2000).

Malonyl-CoA is transferred next to an acyl carrier protein (ACP), followed by subsequent decarboxylation of the malonyl moiety and condensation of $\mathrm{C} 1$ of an acetate and $\mathrm{C} 2$ of the malonyl group on ACP. A sequence of reduction, dehydration and reduction again results in the formation of palmitic acid (16:0) and stearic acid (18:0) bound to ACP (Somerville et al. 2000). The introduction of the first double bond into 16:0-ACP and 18:0-ACP leads to the formation of either 16:1-ACP or 18:1-ACP and is catalysed by a stearoyl-ACP-desaturase (Shanklin and Cahoon 1998). These resulting FAs $(16: 0,16: 1,18: 0,18: 1)$ are then either used for the synthesis of plastidial glycerolipids or released into the cytoplasm where they get activated to CoA esters forming the acyl-CoA pool and are suitable for further reactions, such as elongation or incorporation into glycerol or sphingolipids (Moore 1982; Millar et al. 2000).

According to the endosymbiosis theory, the biosynthetic pathway of lipids within plastids is called prokaryotic biosynthetic pathway. FAs produced via the prokaryotic pathway are transferred to the $s n-1$ and $s n-2$ position of glycerinealdehyde-3-phosphate (G3P), which is then converted to the phospholipid phosphatidic acid (PA). The substrate and positional specificities of the plastidial acyltransferases are responsible for the prokaryotic distribution of FAs at the glycerol backbone of lipids (Frentzen 1993). Two specific acyltransferases were identified transferring FAs attached to ACP to G3P. The reaction of the acyl-ACP:glycerol-3-phosphate acyltransferase (GPAT) leads to the formation of lysophosphatidic acid (LPA) which is further converted to PA by the membrane-localised lysophosphatidic acid acyltransferase (LPAT). Thereby 18:0 is exclusively bound to the $s n-1$ position of the glycerol backbone, whereas at the $s n-2$ position 16:0 is bound (Ohlrogge et al. 1991).

PA can further be metabolised to phosphatidylglycerol (PG) and diacylglycerol (DAG), which is in turn the initial lipid for the synthesis of glycolipids. The transfer of different head groups to DAG leads to the formation of MGD, DGD and SQD (Joyard et al. 
1993). The FAs bound to these glycolipids can further be modified by membrane bound desaturases, which introduce double bonds into the acyl residues (Heinz 1993; Browse and Somerville 1994).

The acyl-CoA-esters outside the plastids are incorporated into lipids within the endoplasmatic reticulum (ER). This part is called eukaryotic biosynthetic pathway. The selectivity of cytoplasmatic acyltransferase results in a so-called eukaryotic FA distribution at the glycerol backbone of lipids. Therefore 18:1 and not 16:0 is mainly bound to the $s n-2$ position of the glycerol backbone. PA and DAG are synthesised via the same reactions as within the plastids, but in contrast to acyl-ACPs acyl-CoAs function as substrate for GPAT and LPAT. DAG is further converted to the phospholipids phosphatidylethanolamine (PE) and phosphatidylcholine (PC). Other phospholipids, like phosphatidylinositol (PI), phosphatidylserine (PS) and phosphatidylglycerine (PG) are synthesised via the so-called cytidine diphosphodiacylglycerol (CDP-DAG)-pathway (Carman 2005). In this pathway PA gets activated forming CDP-DAG, to which the hydroxyl of an alcohol like inositol or serine is linked. For the synthesis of CL, which is located in the mitochondria, one molecule of PG is linked to CDP-DAG by a diphosphatidylglycerol synthase (Schlame et al. 2000). The synthesis of glycolipids is exclusively exerted within the plastid envelope. The biosynthesis involves the formation of PA in the plastids and at the ER (Browse and Somerville 1994). The DAG molecules can either derive from the synthesis within the plastids or at the ER and therefore the distribution of FAs at the glycerol backbone can be both, prokaryotic and eukaryotic (Browse et al. 1986).

But recently, an alternative pathway of glycolipid synthesis in the red alga Cyanidioschyzon merolae was suggested (Sato and Moriyama 2007). Genomic analysis revealed that $C$. merolae lacks the acyl-lipid desaturases of cyanobacterial origin as well as the stearoyl acyl-carrier-protein desaturase, which are the major desaturases in plants and green algae. With labelling experiments the authors could show, that this red alga synthesises MGD via a "coupled pathway" using plastidic derived 16:0 and ER derived LA.

At last, the synthesis of TAG happens at the ER-membrane within the cytoplasm (Voelker and Kinney 2001). The precursor is DAG, to which a third acyl residue is bound at the sn-3 position. The transfer is either catalysed by a diacylglycerol acyltransferase (DAGAT) with the substrate coming from the cytoplasmatic acylCoA 
pool or by a phospholipid diacylglycerol acyltransferase (PDAT) with the substrate coming from the PC pool of the ER (Dahlqvist et al. 2000; Voelker and Kinney 2001).

\subsection{Biosynthesis of polyunsaturated fatty acids (PUFAs)}

In contrast to higher plants, in which the number of double bonds in FAs does not comprises up to three, algae and animals can introduce up to eight double bonds into FAs (Linko and Karinkanta 1970; Mansour et al. 1999).

The introduction of double bonds into the acyl chain is catalysed by desaturases, which show different substrate specificities. The plastid-localised soluble desaturase acts on acyl chains bound to ACP and is termed acyl-ACP-desaturase. The stearoyl-ACP $\Delta^{9}$ desaturase introduces a double bond into stearic acid resulting in $18: 1^{\Delta 9}$-ACP, as described in the previous section (Shanklin and Cahoon 1998). Except from the soluble acyl-ACP desaturase family, all other desaturases are integral membrane proteins with either acyl-lipid substrates or acyl-CoA substrates. In plants and cyanobacteria acyllipid-desaturases introduce double bonds into FAs whereas in some yeast and animal cells acyl-CoA-desaturases catalyse the introduction of double bonds into FAs (Somerville et al. 2000). Recently the first acyl-CoA-dependent desaturase was identified from the microalga Ostreococcus tauri (Domergue et al. 2005).

The initial substrate for the PUFA biosynthetic pathway is $18: 1^{9 Z}$ after its incorporation into PC. Then a $\Delta^{12}$-desaturase introduces the second double bond, resulting in LA, which may be further desaturated by a $\Delta^{15}$-desaturases to ALA. These modified FAs are then exchanged by other 18:1 acyl residues and may be released into the cytoplasm as acyl-CoA derivatives. In the cytoplasm the acyl-CoA derivatives are extended to about C-26 or even longer acyl chains by specific elongase complexes. These elongase reactions have several important differences between that of FAS reactions in the plastids: the elongases are membrane-bound and ACP is not involved in the elongation process (Somerville et al. 2000). In plants and mammals it is believed that FA elongation is a four-step process with the condensation of malonyl-CoA with a longchain acyl-CoA as the initial reaction (Parker-Barnes et al. 2000). The initial condensation in plants is catalysed by a $\beta$-ketoacyl-CoA synthase (KCS). Surprisingly, the corresponding condensing enzymes in yeast, the yeast FA elongase system (ELO) (Toke and Martin 1996), do not share any sequence similarities to plant KCS and also other cloned fungal, algal, moss and mammalian PUFA elongases share homology with yeast ELO sequences and not with plant KCS sequences (Zank et al. 2002). 
Further desaturation steps lead to the synthesis of FAs with more than 3 double bonds. During the biosynthesis of VLCPUFA a continuous exchange between the biosynthetic steps of CoA-bound and lipid-linked acyl residues takes place (Fig. 1). This lipid/CoA pathway requires an efficient acyl exchange between phospholipids and the acyl-CoA pool and is supposed to be the rate limiting step in the VLCPUFA synthesis in plants. In contrast, the CoA pathway does not require a shuttling of acyl residues between acyl-CoA pool and phospholipids. Acyl-CoA specific desaturases allow a subsequent desaturation and elongation of FA, which exclusively takes place in the acyl-CoA pool.

The most important VLCPUFAs are arachidonic acid (AA, 20:4n-6), eicosapentaenoic acid (EPA, 20:5n-3), and docosahexaenoic acid (DHA, 22:6n-3). The pathway shown in Fig. 1 is regarded as the biosynthetic pathway of VLCPUFAs found in all eukaryotes. According to the position of the closest double bond to the omega end of the PUFAs, two pathways are distinguishable: the $\omega 3$ and $\omega 6$-pathway. In addition, some algae can produce EPA using another pathway, termed the $\triangle 8$-alternative pathway, in which LA is converted to $20: 3^{(8 Z, 11 Z, 14 Z)}$ (Qi et al. 2002). First, LA is elongated to $20: 2^{(11 Z, 14 Z)}$ by a $\Delta 9$-elongase and then, a $\Delta 8$-desaturase introduces the third double bond, resulting in $20: 3^{(8 Z, 11 Z, 14 Z)}$, which can sequentially be converted to AA and then EPA (Fig. 1). Another alternative pathway of PUFAs is the Sprecher pathway which was found for mammalian cells (Sprecher 2000) and is also considered to be present also in some species of the class Dinophyceae (Henderson 1999; Bergé and Barnathan 2005). In this pathway DHA is not directly obtained by elongation and desaturation of EPA, but formed via $\beta$-oxidation of $24: 6^{(6 Z, 9 Z, 12 Z, 15 Z, 18 Z, 21 Z)}$, which is synthesised by elongation and desaturation of $22: 5^{(7 Z, 10 Z, 13 Z, 16 Z, 19 Z)}$ at the ER and then transported to the peroxisomes (Fig. 1). 


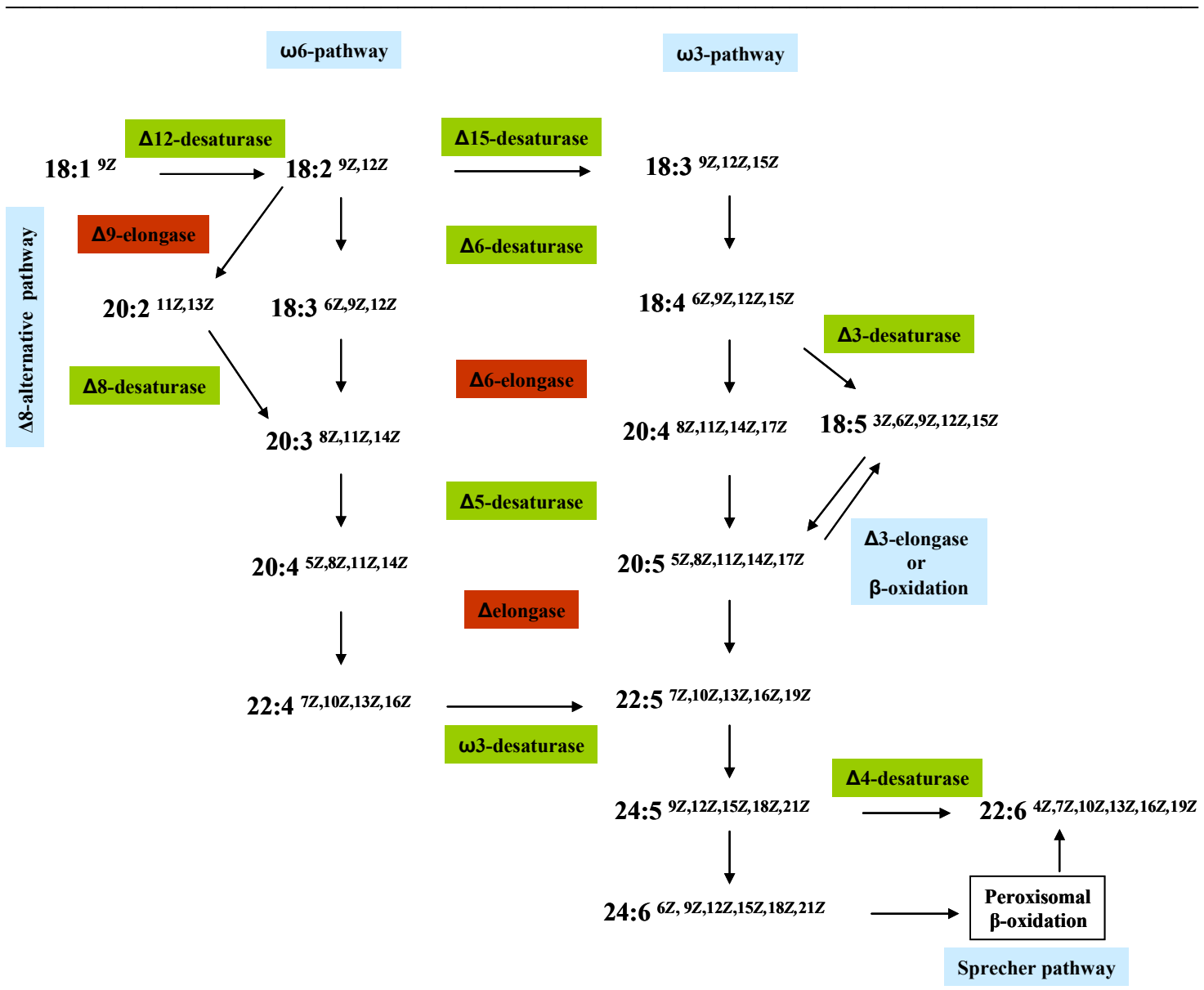

Figure 1. Schematic representation of VLCPUFA biosynthesis via the $\omega 6$ - and $\omega 3$ pathway. Additional route for the synthesis of $\mathrm{AA}$ is the $\Delta 8$-alternative pathway. Additional route for the synthesis of DHA is the mammalian Sprecher pathway. Desaturase-catalysed steps are coloured in green, elongase catalysed steps in red (Napier 2007).

Besides the standard FA biosynthetic pathway consisting of oxygen dependent desaturation and elongation reactions VLCPUFAs can be synthesised via an anaerobic pathway catalysed by polyketide synthases (PKS) (Metz et al. 2001). PKSs carry out similar reactions as FAS and use acyl carrier protein (ACP) as covalent attachment site for the growing chain. In contrast to the standard VLCPUFA synthesis which requires approximately 30 enzyme activities and almost 70 reactions, the PKSs consists of a single, multidomain enzyme, carrying out the complete cycle of reduction dehydration and reduction (Bentley and Bennett 1999). Often this cycle is abbreviated resulting in highly derivatised carbon chains, such as aflatoxins and antibiotics. But some marine organisms, like Schizochytrium, produce EPA and DHA via the PKS pathway (Metz et al. 2001). 
Over the last years, a multitude of interesting PUFAs from microalgae were identified. One of these PUFAs is $18: 5 n-3$, which was first found in the dinophyte Prorocentrum minimum (Joseph 1975). Until now the biosynthesis of 18:5n-3 is not fully understood. Joseph et al. proposed two ways: 18:5n-3 could either be synthesised by desaturation $\left(\Delta^{3}\right.$-desaturase) of $18: 4 n-4$ or by $\beta$-oxidation of EPA (Fig. 1). Upon a following elongation step ( $\Delta^{3}$-elongase) of $18: 5 n-3$, EPA is obtained. This pathway may be a fourth alternative to synthesise EPA.

\subsection{Engineering of VLCPUFA synthesis in plants}

Beside fish-farming and aquaculture of algae the production of VLCPUFAs in annual oilseeds by biotechnology would provide a reliable and renewable source (Drexler et al. 2003; Abbadi et al. 2004). Thus, the implementation of the biosynthetic pathways of VLCPUFAs from microalgae into oilseeds would be required. First results were obtained by Qi et al. 2004 who constitutively expressed an elongase from the microalgae Isochrysis galbana and two desaturases from the microalgae Euglena gracilis and the fungus Mortierella alpine in leaves of the model plant Arabidopsis thaliana. Further on Abbadi et al. (2004) transformed tobacco and flax with different combinations of desaturases and elongases resulting in only modest amounts of AA and EPA in the seeds of the plants. Recently Wu et al. (2005) reported about the transgenic oil crop Brassica juncea accumulating AA in levels up to $25 \%$ and EPA in levels up to $15 \%$. These high yields of VLCPUFAs were reached by a stepwise metabolic engineering strategy.

However, several drawbacks and/or bottlenecks have been identified implying the need for additional enzymes to be transformed to transgenic plants (Drexler et al. 2003; Abbadi et al. 2004). One main problem is the acyl-shuttling between PC and CoA-pools during VLCPUFA synthesis catalysed by a specific acyltransferase, which the transgenic plants lack (Abbadi et al. 2004; Robert 2006). To circumvent the bottle neck two approaches were suggested: the implementation of desaturases, which act on acylCoAs like elongases or the isolation and implementation of PUFA specific acyltransferases, which shuttle VLCPUFAs between the two pools. Therefore the first step would be the systematic identification and analysis of the additional pathways in a large and divergent class of organisms. Then, genes of the enzymes involved can be 
isolated, followed by the biochemical characterisation of the recombinant enzymes in an eukaryotic host like yeast. Particularly the various acyltransferases, which are responsible for the transfer of PUFAs into TAGs, have to be identified.

The second approach to gain high proportions of EPA and DHA in oilseed crops is the implementation of PKS pathways into plants (Damude and Kinney 2007). Although several PKS from traustochytid species have been cloned and characterised (Metz et al. 2001) there are no published studies to date of successful transfer of PKS pathway in higher plants (Damude and Kinney 2007).

\section{Oxylipin formation}

Lipid peroxidation is an important process in lipid metabolism. Lipid peroxides also play an important role as precursors for the synthesis of signal molecules and are collectively called oxylipins.

In plants, moss and algae oxylipins are involved in a wide spectrum of mechanisms to defend themselves against biotic and abiotic stress like drought, mechanical wounding, UV-radiation, temperature and pathogens (Wendel and Jüttner 1996; Schilmiller and Howe 2005a; Senger et al. 2005a; Singh et al. 2005). The major biosynthetic pathway involved in generating oxylipins is the lipoxygenase pathway (Bleé 2002). The first enzyme of this pathway is a lipoxygenase (LOX). It forms a family of non-heme iron containing FA dioxygenases, which catalyse the regio- and stereospecific insertion of molecular oxygen into PUFAs, which leads to the formation of FA hydroperoxides (Brash 1999). LOXs are widely distributed in nature and occur in a great variety ubiquitously in eukaryotes like in higher plants and mammals (Thiele et al. 1990; Feussner and Wasternack 2002). Recently data about LOXs from lower organisms, like coral, moss, algae and bacteria, have been published (Beneytout et al. 1989; Koljak et al. 1997; Senger et al. 2005a; Vidal-Mas et al. 2005). But still, only little in known about the origin and function of these enzymes. 


\subsection{The LOX pathway in plants}

In plants the PUFAs raughanic acid $\left(16: 3^{7 Z, 10 Z, 13 Z}\right)$ LA and ALA present the main substrates for the LOX reaction. Alternatively they may be metabolised by $\alpha$-dioxygenases ( $\alpha$-DOX). The corresponding hydroperoxides form the central branch point of the LOX pathway (Bleé 2002; Liavonchanka and Feussner 2006). The hydroperoxides can further be metabolised in at least six different reactions sequences (Fig. 2). These pathways are again named after their first enzyme: (1) the allene oxide synthase (AOS) pathway, where an unstable allene oxide is formed, which can be hydrolysed non-enzymatically to $\alpha$ - and $\gamma$-ketols and racemic oPDA (Hamberg 1988). The allene oxides of 13-HPOTE or 11-HPHT may be metabolised by an allene oxide cyclase (AOC) to chiral (9S,13S)-12-oxo phyto-9,13-dienoic acid (oPDA) (Ziegler et al. 2000) or dinor oPDA, which can further be converted to the phytohormone jasmonic acid (JA), (2) the epoxy alcohol synthase (EAS) pathway results in the intramolecular rearrangement of hydroperoxy FAs to epoxy hydroxy FAs (Hamberg 1999), (3) the peroxidase activity of LOX leads to the formation of ketodienes at low oxygen pressure (Kühn et al. 1991), (4) the FA hydroperoxide lyase (HPL) pathway which leads to the formation of short chain aldehydes and the corresponding $\omega$-oxo fatty acids , (5) divinyl ether synthase (DES) pathway, where divinyl ether like colneleic acid and colnelenic acid are formed (Grechkin 1998) and (6) the peroxygenase (POX) pathway leads to the formation of epoxy- or dihydroxy fatty acids (Bleé 1998). Through chemical reduction the hydroperoxides can also be reduced to hydroxides.

Over the last years a lot of information about the role of the LOX pathway in environmental stresses and its involvement in developmental processes were obtained (Müller and Brodschelm 1994; Kühn et al. 2005b; Schilmiller and Howe 2005a). Though, the activation of LOXs and the induction of LOX gene expression upon wounding as well as the involvement of oxylipins in plant defence reactions were shown in several studies (Göbel et al. 2003; Halim et al. 2006). After infection of monoand dikotyledones by phytopathogens, an increased LOX expression and in parallel an increased activity of LOXs was detectable (Feussner and Rosahl 2005). Furthermore jasmonates and their precursors are produced after mechanical wounding through insects resulting in the expression of proteinase inhibitors in the affected tissues (Wasternack and Hause 2002). 


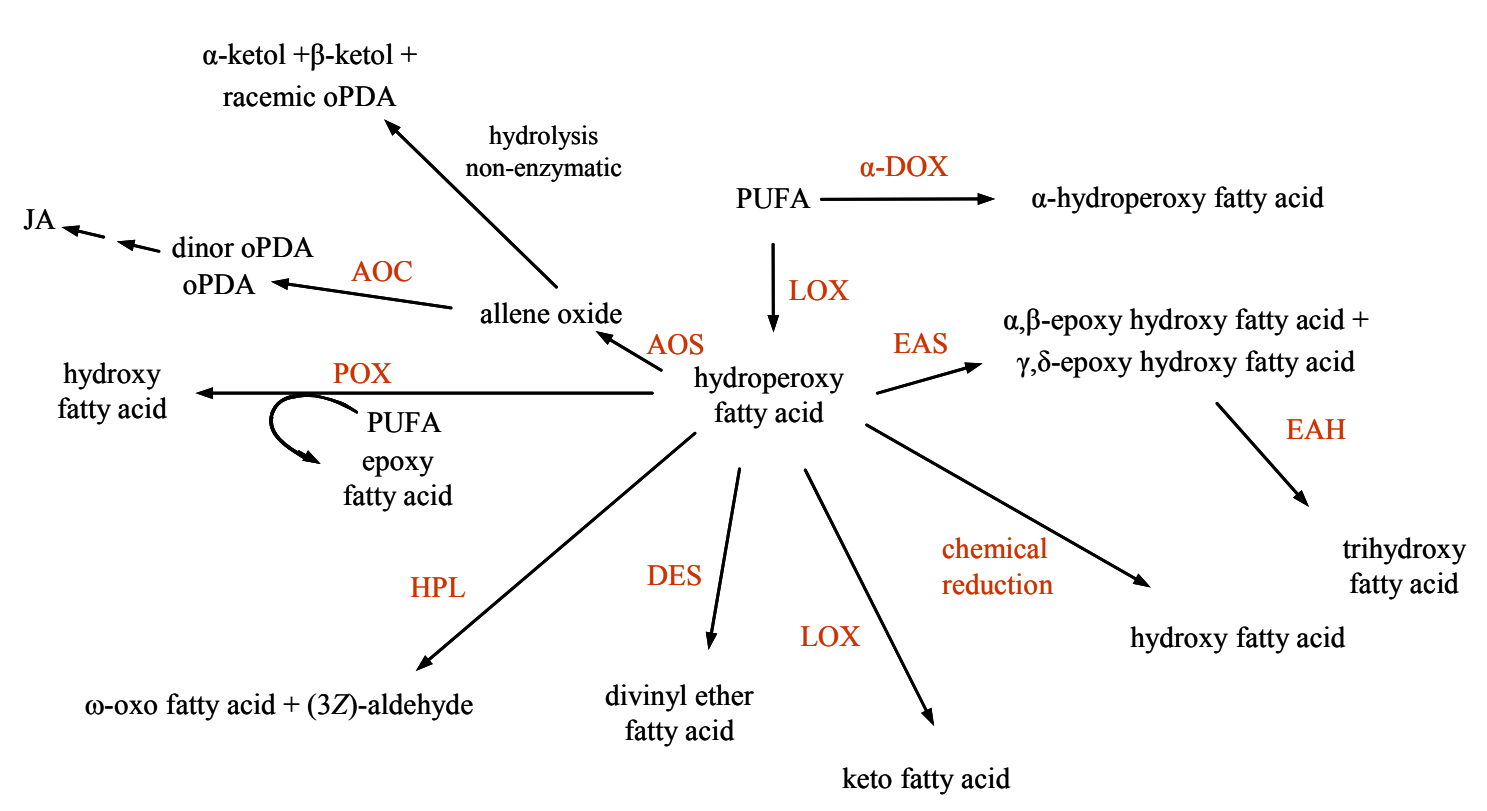

Figure 2. Schematic overview of the plant LOX pathway. Conversion of hydroperoxide by a broad range of enzymes or by chemical reduction leads to the production of different oxylipins. AOC, allene oxide synthase, AOC, allene oxide cylcase, POX, peroxidase, HPL, hydroperoxide lyase, DES, divinyl ether synthase, EAS, epoxy alcohol synthase, EAH, epoxy alcohol hydrolase.

\subsection{Oxylipin formation in algae}

Prokaryotic and eukaryotic algae are well known as sources of biologically active compounds (Cannell 1993). Many of these compounds such as alcohols, alkanes, aldehydes, ketones and branched hydrocarbons may derive from the primary products of LOX activity (Watson 2003). However, most studies about oxylipin formation in algae concentrate on macrophytic marine algae, as their use in human diet and medicine has long tradition (Gerwick 1999a). Especially macrophytic red algae (Rhodophyta) are known for their unique metabolism of FAs to oxylipins. For example the red alga Gracilariopsis lemaneiformis was found to be a rich source of novel oxylipins, like $(5 Z, 8 Z, 10 E, 12 S, 14 Z)$-hydroperoxy-5,8,10,14-eicosatetraenoic acid ((12S)-HPETE) and $(5 Z, 8 Z, 10 E, 12 R, 13 S, 14 Z)$-dihydroxy-5,8,10,14-eicosatetraenoic acid $\quad((12 R, 13 S)$ diHETE, Gerwick, 1991). Therefore G. lemaneiformis became the first marine alga in which detailed studies were undertaken to understand the mechanism of oxylipin formation of algae (Gerwick 1994). But also macrophytic brown and green algae were identified as oxylipin producers (Bernart et al. 1993; Kousaka et al. 2003). For the 
chlorophyte Ulva conglobata two LOX derived hydroperoxides could be detected: $(9 R, 10 E, 12 Z)-9$-hydroperoxy-10,12-octadecadienoic acid ((9R)-HPODE) and $(9 R, 10 E, 12 Z, 15 Z)-9$-hydroperoxy-10,12,15-octadecadienoic acid ((9R)-HPOTE) (Akakabe et al. 2002). In addition several other odorous compounds were found like (8Z)-8-heptadecenal, $(8 Z, 11 Z, 14 Z)-8,11,14$-heptadecatrienal. The identified oxylipins lead to the hypothesis that the PUFAs like LA and ALA were converted to 2-, 9-, and 13-hydroperoxides which were then subsequently converted into C-17, C-9 and C-6 aldehydes.

With growing interest in harmful algal blooms and in particularly in understanding blooms dynamics, toxicity and effects on consumers and competitors, the elucidation of synthesis and function of secondary metabolites of marine microalgae became more and more important (Paul et al. 2006).

Nevertheless, still little is known about the role of oxylipin metabolism in algal biochemistry and physiology. Due to the increasing sequence information from genome sequencing projects not only biochemical but also molecular tools may be used for the identification and isolation of new LOXs and further enzymes from the LOXs pathway.

\section{Oxylipin formation in microalgae}

The cyanobacterium Oscillatoria spp was the first prokaryotic alga for which LOX activity was described (Beneytout et al. 1989). The enzyme isolated was characterised as an unspecific 9/13-LOX. Further, in Anabaena flos-aquae $f$. flos aquae two hydroperoxy FAs in $R$-configuration $((9 R)$-HPODE and $(9 R)$-HPOTE) were isolated, which leads to the suggestion that this organism might contain a (9R)-LOX (Murakami et al. 1992). Another study reports about the formation of the triol $(9 S, 10 E, 12 R, 13 S)$ 9,12,13-trihydroxy-10,15-octadecadienoic acid (malyngic acid) in the cyanobacterium Lyngbya majuscule (Cardellina and Moore 1980). The authors suggested, that malyngic acid might be synthesised via $(9 S, 10 E, 12 S, 13 S, 15 Z)$-12,13-epoxy-9-hydroxy-10,15octadecadienoic acid, which in turn might be formed by a rearrangement of (9Z,11E,15Z)-13S-hydroperoxy-9,11,15-octadecatrienoic acid ((13S)-HOTE). The hydroperoxide $(13 S)$-HOTE, in turn, might originate from the action of a LOX on ALA. One interesting aspect was the chemical defence of diatoms against copepods, which has been studied intensively the last years (Miralto et al. 1999; Pohnert et al. 2002). It was shown, that damaged or wounded diatoms produce a wide range of different 
biologically active oxylipins, which lead to abortive and teratogenic effects in zooplankton herbivores (Miralto et al. 1999; Adolph et al. 2004; d'Ippolito et al. 2004; Pohnert 2005a). Wendel and Jüttner (1996) could increase the concentration of hydrocarbons and unsaturated aldehydes of several diatoms, e.g. Asterionella formosa and Gomphonema parvulum, when the LOX reaction was initiated by osmotic stress. Further on, the presence of short-chain aldehydes and other C16-FA derived oxylipins, like $(6 Z, 10 E, 12 Z)-9$-hydroxy-6,9,12-hexadecatrienoic acid, in the diatom Thalassiosira rotula lead to the assumption, that these algae contain at least one LOX. However, in the recently published genome sequence of the closely related diatom Thalassiosira pseudonana (Armbrust et al. 2004) no LOX was annotated which may indicate that LOX enzymes may not be distributed ubiquitously in microalgae.

However, until now only one LOX of a eukaryotic microalga, the green algae Chlorella pyrenoidosa, was purified and characterised in more detail (Vick and Zimmerman 1989). It turned out to produce 9- and 13-HPODE isomers in an equal ratio, whereas the product distribution ratio was not dependent on $\mathrm{pH}$.

With regard on the huge phylogenetic diversity of microalgae almost nothing is known about the occurrence of LOX derived oxylipins and their function.

\section{Aim of the project}

Hitherto no systematic analysis has been carried out on a large scale basis on the profiles of lipids or FAs in microalgae. Though, the characterisation of fatty acid profiles of all available microalgal strains of the culture collection of microalgae in Göttingen (SAG) was the main objective of this work. The SAG is a living resource of culture material of microalgae serving research, teaching and biotechnology communities mainly throughout Europe. The collection primarily comprises microscopic algae and cyanobacteria from freshwater or terrestrial habitats, but marine algae and microscopic stages of some seaweed are also available. The collection covers an extremely broad taxonomic range with algae and cyanobacteria originating from an equally diverse variety of geographical and ecological niches. At present, 2151 culture strains representing 486 genera and 1228 species are available. The focus of this work is set on long chain FAs (C14-C24), which are analysed via appropriate techniques. (gas- 
chromatography and mass spectrometry). The large number of data obtained shall be added into a database, which finally contains all microalgal strains and their corresponding FA profile. As an additional aspect of this investigation the lipid screening can be examined for its suitability as a chemotaxonomic marker. Further on the data obtained of FA profiles will then be used to identify microalgal strains, which harbour new or interesting PUFAs, like 18:5n-3 in high amounts. A maximum accumulation of the desired FAs will be traced by variations of growth conditions (e.g. light, temperature and composition of the medium). Finally the algal strains identified and optimised for the production of FAs will be examined for the corresponding biosynthetic pathways. In order to identify novel desaturases and elongases involved in the synthesis of certain FAs, analysis of their biosynthesis by in vivo labelling studies shall be performed.

The last objective of this thesis is the analysis of the metabolism of PUFAs, in particular the oxylipin formation, in selected algae. For this purpose, the SAG microalgal strains are analysed for their hydro(pero)xy FA and volatile contents. In parallel, the isolation of genes which are involved in the oxylipin pathway is aimed. Therefore database searches will be performed to find sequences encoding LOXs in microalgae, which catalyse the initial step of the oxylipin pathway. The sequences identified will be isolated from the appropriate microalgae strain and further on the LOXs will be biochemically characterised. In parallel the function of the LOXs and the oxylipin pathway within these microalgae are going to be analysed in vivo. 


\section{Material and Methods}

\section{Chemicals}

If not mentioned otherwise chemicals were purchased from Sigma-Aldrich (München) or Carl Roth Co (Karlsruhe). All solvents of high performance liquid chromatography (HPLC) grade for the analytical methods were purchased from Acros (Geel, Belgium) or Baker (Griesheim). For the analysis of acyl CoA species (10.5) solutions with HPLC gradient grade were purchased from VWR (Darmstadt). If not mentioned otherwise water was used as double distilled water $\left(\mathrm{ddH}_{2} \mathrm{O}\right)$.

\section{Standards}

Fatty acid methyl ester mixture (F.A.M.E. Mix, C4-C24)

Tripentadecanoate

Linoleic acid methyl ester
Sigma, München Sigma, München

Sigma, München

\section{Kits/systems}

NucleoSpin ${ }^{\circledR}$ Plant kit

NucleoSpin TM Plasmid kit

ExpandTM High Fidelity PCR System

DNA and Gel Band Purification Kit

$\mathrm{pCR}^{\circledR} \mathrm{T} 7 / \mathrm{TOPO}^{\circledR} \mathrm{NT}$

$\mathrm{pGEM}^{\circledR}$-T ligation Kit

ABI Prism BigDye Terminator Cycle

Sequencing Ready Reaction Kit v1.1
Macherey\&Nagel, Düren

Macherey\&Nagel, Düren

Roche Diagnostics, Mannheim

GE Healthcare, München

Invitrogen, Karlsruhe

Promega, Heidelberg

Applied Biosystems, Foster City (USA)

\section{Enzymes}

All restriction enzymes were purchased from Fermentas (St. Leon-Rot) and used according to manufacturer's instruction. 


\section{Bacterial strains}

E. coli XL1Blue: (Bullock et al. 1989)

recAlendA1gyrA96 thi-1hsdR17 supE44 relA1lac[F 'proAB lac9zM15 Tn19(Tetr)]

(Stratagene, Heidelberg)

E. coli Rosetta (DE3)

FompT hsd $S_{B}\left(r_{B}{ }^{-} m_{B}^{-}\right)$gal dcm (DE3)pRARE ${ }^{2}\left(\mathrm{Cam}^{2}\right)$ (Invitrogen, Karlsruhe)

E. coli One Shot TOP10F'

$\mathrm{F}^{\prime}\left[\right.$ lacl $^{q}, \operatorname{Tn} 10\left(\right.$ Tet $\left.\left.^{\mathrm{R}}\right)\right] m c r \mathrm{~A} \quad \Delta(m r r-h s d \mathrm{RMS}-m c r \mathrm{BC}) \Phi 80$ lacZ $\Delta$ lacX74 recA1 araD139

$\Delta($ ara-leu $) 7697$ galU galK rpsL $\left(\mathrm{Str}^{\mathrm{R}}\right)$ end $\mathrm{A} 1$ nup $\mathrm{G}$ (Invitrogen, Karlsruhe)

E. coli BL21(DE3):

$\mathrm{F}^{-}$ompT hsd $S_{\mathrm{B}}\left(\mathrm{r}_{\mathrm{B}}^{-} \mathrm{m}_{\mathrm{B}}^{-}\right)$gal dcm (DE3) (Invitrogen, Karlsruhe)

E. coli HMS174

$\mathrm{F}^{\prime}, \operatorname{rec} A, r_{K 12-m K 12}+$, Rif $^{3} \mathrm{DE} 3$

\section{Hardware/Equipment}

Kontron 340 Recorder

Oxygen-electrode

Lyophilisator

High performance liquid chromatography (HPLC)

HPLC/mass spectrometry

Gas chromatograph (GC)

GC/mass spectrometry

Thermocycler
Kontron, München

Rank Borther, Bottisham, UK

Leybold-Heraeus GmbH Köln

Agilent, Waldbronn

Thermo Finnigan, Darmstadt

Agilent, Waldbronn

Agilent, Waldbronn

Eppendorf, Wesseling-Berzdorf

\section{Strains and culture conditions of algae used}

\subsection{Preparation of SAG microalgae strains}

The SAG comprises 2231 strains representing 510 genera and 1273 species (Fig. 3). About $77 \%$ of all strains are representatives of Chlorophyta and Streptophyta and $8 \%$ belong to the Cyanophyta. Euglenophyta are represented with $6 \%$, Stramenopiles with $5.3 \%$, Rhodophyta with $3 \%$ and others with $0.7 \%$. 
For analysis of algal strains cells were harvested in the stationary phase and stored at $-20{ }^{\circ} \mathrm{C}$. Depending on culture and growth condition of the algal cultures the stationary phase was reached after different periods of culture, ranging from three months to one year. Before FA extraction (10.2) the algae were lyophilised for 2 days until the cell pellets were totally dry.

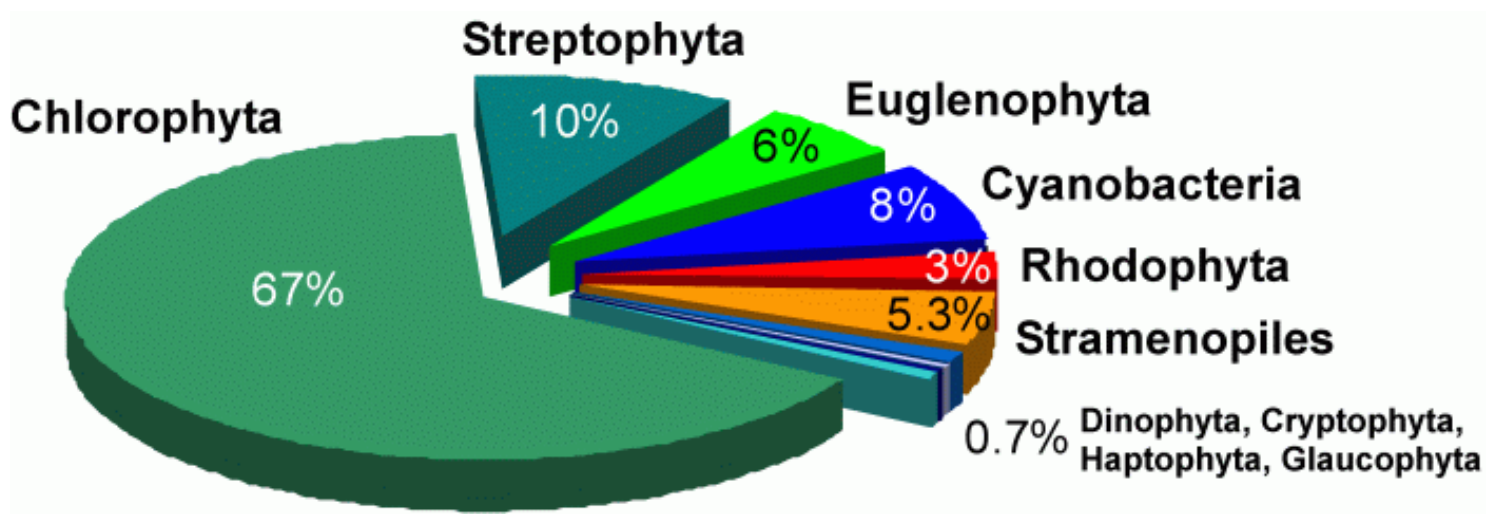

Figure 3 Diversity of taxonomic groups of microalgae maintained in the SAG. (Diagram is kindly provided by Prof. T. Friedl)

\subsection{Cultivation of Prymnesium parvum SAG 127.79 and P. saltans SAG 18.97}

P. parvum SAG 127.79 and $P$. saltans SAG 18.97 were cultivated at $20^{\circ} \mathrm{C}$ in $100 \mathrm{ml}$ Erlenmeyer flasks with $50 \mathrm{ml} 1 / 2$ SWES medium according to the standard protocols of the SAG (http://www.epsag.uni-goettingen.de/html/culturemedia.html\#listofmedia).

\subsection{Cultivation of Nostoc punctiforme PCC 73102 and Nostoc sp. SAG 25.82}

Nostoc punctiforme PCC 73102, Nostoc sp. SAG 25.82 and Nostoc sp. PCC7120 were cultivated in BG11 medium for cyanobacteria according to the standard protocols of the SAG (http://www.epsag.uni-goettingen.de/html/culturemedia.html\#listofmedia) at $20{ }^{\circ} \mathrm{C}$ using 11 conical flasks shaking at $120 \mathrm{rpm}$. After 3 weeks of growing the cells were harvested by centrifugation at $3220 \mathrm{x} g$ for $10 \mathrm{~min}$ and the pellet was stored at $-20^{\circ} \mathrm{C}$.

\section{Molecular analysis}

For all molecular methods standard protocols were used according to (Ausubel et al. 1993). Methods different from the standard protocols are described in the following section. 


\subsection{Isolation of DNA}

Genomic DNA was isolated from approximately $50 \mathrm{mg}$ of frozen cell material using the NucleoSpin ${ }^{\circledR}$ Plant kit (Macherey\&Nagel, Düren) according to the manufacturer's instructions.

\subsection{Isolation of plasmid-DNA}

The isolation of Plasmid DNA from E. coli was performed with liquid cultures according to (Birnboim and Doly 1979). Plasmid preparations in small scale were done by using the NucleoSpinTM Plasmid"-Kit (Macherey \& Nagel, Düren) following the manufacturer's instructions.

\subsection{Isolation of $N p L O X 1$ and $N p L O X 2$ from $N$. punctiforme PCC 73120}

The genes encoding two LOX-like proteins were isolated from genomic DNA-templates by the polymerase chain reaction (PCR) (Saiki et al. 1988) using ExpandTM High FidelityPCR-System (Roche Diagnostics, Mannheim) and the following primer combinations: NpLOX1:

NpLOX40F, 5'-ATGACTGCTTTATCACCAGATCATTCAATCAGTTCA-'3

NpLOX40R, 5'-TCAGATATTGATGCTCTGAGGAATTTTA-'3

NpLOX2:

NpLOX50F, 5'-ATGAAACCATACCTCCCTCAGAATGAT-'3

NpLOX50R, 5'-TCACACGCTAATACTATTTGACACAAG-'3.

The amplification was carried out in a thermocycler (Mastercycler personal, Eppendorf) using the following PCR program:

\begin{tabular}{|c|c|c|}
\hline & $94^{\circ} \mathrm{C}$ & $2 \min$ \\
\hline $10 \times$ & $\begin{array}{l}94^{\circ} \mathrm{C} \\
58^{\circ} \mathrm{C} \\
72^{\circ} \mathrm{C}\end{array}$ & $\begin{array}{l}30 \mathrm{sec} \\
30 \mathrm{sec} \\
1: 30 \mathrm{~min}\end{array}$ \\
\hline $15 \times$ & $\begin{array}{l}94^{\circ} \mathrm{C} \\
58^{\circ} \mathrm{C} \\
72^{\circ} \mathrm{C}\end{array}$ & $\begin{array}{l}30 \mathrm{sec} \\
30 \mathrm{sec} \\
1: 30 \mathrm{~min}+5 \mathrm{sec} / \mathrm{cycle}\end{array}$ \\
\hline & $72^{\circ} \mathrm{C}$ & $3 \mathrm{~min}$ \\
\hline
\end{tabular}


PCR products were electrophoretically separated on a $1 \%(\mathrm{w} / \mathrm{v})$ agarose gel and bands of the correct size were cut out and extracted from the gel with the DNA and Gel Band Purification Kit (GE Healthcare, München) according to manufacturer's instructions. The obtained fragments LOX1 and LOX2 were cloned into the bacterial expression-vector pEXP5-NT/TOPO $^{\circledR}$ (Invitrogen, Karlsruhe) and transformed into E. coli One Shot ${ }^{\circledR}$ TOP10F' cells. The plasmid DNA of correct clones was isolated as described in section 8.2 and the constructs were transformed into One Shot ${ }^{\circledR}$ BL21(DE3) cells following the manufacturer's protocol.

\subsection{Isolation of Peroxidase-LOX fusion protein from Nostoc sp. SAG 25.82}

PCR was carried out in a thermocycler (Mastercycler personal, Eppendorf) using the ExpandTM High Fidelity-PCR-System (Roche Diagnostics, Mannheim) according to manufacturer's instructions. Primer combinations were used as indicated in Fig. 4.

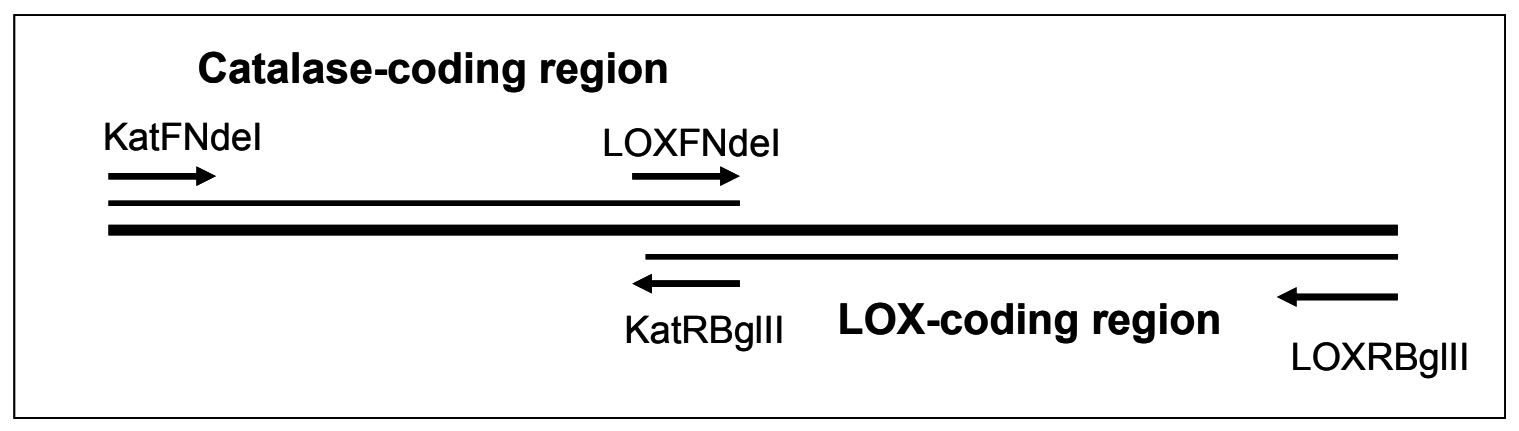

Figure 4. Scheme of the Peroxidase-LOX fusion protein gene and the primers used for the amplification of three fragments. KatFNdeI and KatRBglII for Peroxidase coding region, LOXFNdeI and LOXRBglII for LOX coding region and KatFNdeI and LOXRBglII for the whole gene.

The gene encoding the LOX coding domain was isolated using the following primer combinations:

LOXFNdeI, 5' - ACATATGCAGTATTTGTATGGAAGTAAAGG '-3 (NdeI recognition site)

LOXRBglII, 5'-A site)

The gene encoding the peroxidase coding domain was isolated using the following primer combinations: 
KatFNdeI, 5'-ACATATGGATTTGAATACTTACTTGAAG'-3 (NdeI recognition site)

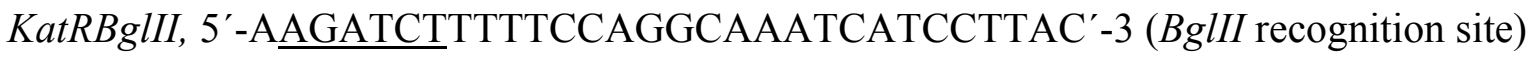

For the amplification of the Peroxidase and LOX domain the following PCR-program was used:

$\begin{array}{rl}94^{\circ} \mathrm{C} & 2 \mathrm{~min} \\ 94^{\circ} \mathrm{C} & 30 \mathrm{sec} \\ 54^{\circ} \mathrm{C} & 30 \mathrm{sec} \\ 72^{\circ} \mathrm{C} & 1: 30 \mathrm{~min} \\ & \\ 94^{\circ} \mathrm{C} & 30 \mathrm{sec} \\ 54^{\circ} \mathrm{C} & 30 \mathrm{sec} \\ 72^{\circ} \mathrm{C} & 1: 30 \mathrm{~min}+5 \mathrm{sec} / \mathrm{cycle} \\ 72^{\circ} \mathrm{C} & 3 \mathrm{~min}\end{array}$

The fragments obtained were purified with the DNA and Gel Band Purification Kit (GE Healthcare, München) and cloned into pGEM-T-vector (Promega, Heidelberg). The fragments were cut out with the restriction enzymes $\mathrm{NdeI}$ and $\mathrm{BglII}$ and then ligated into the expression vector $\mathrm{pET} 15 \mathrm{~b}$ in frame with an N-terminal His-Tag (Novagene, Bad Soden). The vector containing the correct insert was transformed into E. coli Rosetta(DE3) cells.

The gene encoding the Peroxidase-LOX fusion protein was isolated using the primers KatFNdeI and LOXRBglII and the following PCR program

$\begin{array}{ll}94^{\circ} \mathrm{C} & 2 \mathrm{~min} \\ 94^{\circ} \mathrm{C} & 30 \mathrm{sec} \\ 54^{\circ} \mathrm{C} & 30 \mathrm{sec} \\ 72^{\circ} \mathrm{C} & 2: 30 \mathrm{~min} \\ 94^{\circ} \mathrm{C} & 30 \mathrm{sec} \\ 54^{\circ} \mathrm{C} & 30 \mathrm{sec} \\ 72^{\circ} \mathrm{C} & 2: 30 \mathrm{~min}+5 \mathrm{sec} / \mathrm{cycle} \\ 72^{\circ} \mathrm{C} & 3 \mathrm{~min}\end{array}$

The obtained fusion protein fragment, named NspFP, was purified via agarose gel electrophoresis and extracted following the protocol of the DNA and Gel Band Purification Kit (GE Healthcare, München). The fragment was cloned directly into the expression system $\mathrm{pCR}{ }^{\circledR} \mathrm{T} 7 / \mathrm{TOPO}{ }^{\circledR} \mathrm{NT}$ (Invitrogen, Karlsruhe) following manufacturer's instructions. The vector containing the correct insert was transformed into E. coli BL21(DE3) cells following the standard protocol in the manual. 


\subsection{PCR-mutagenesis}

The fragment obtained of the fusion protein contained an insertion of $1300 \mathrm{bp}$ in the peroxidase coding domain, which was removed by PCR mutagenesis as indicated in Fig. 5:
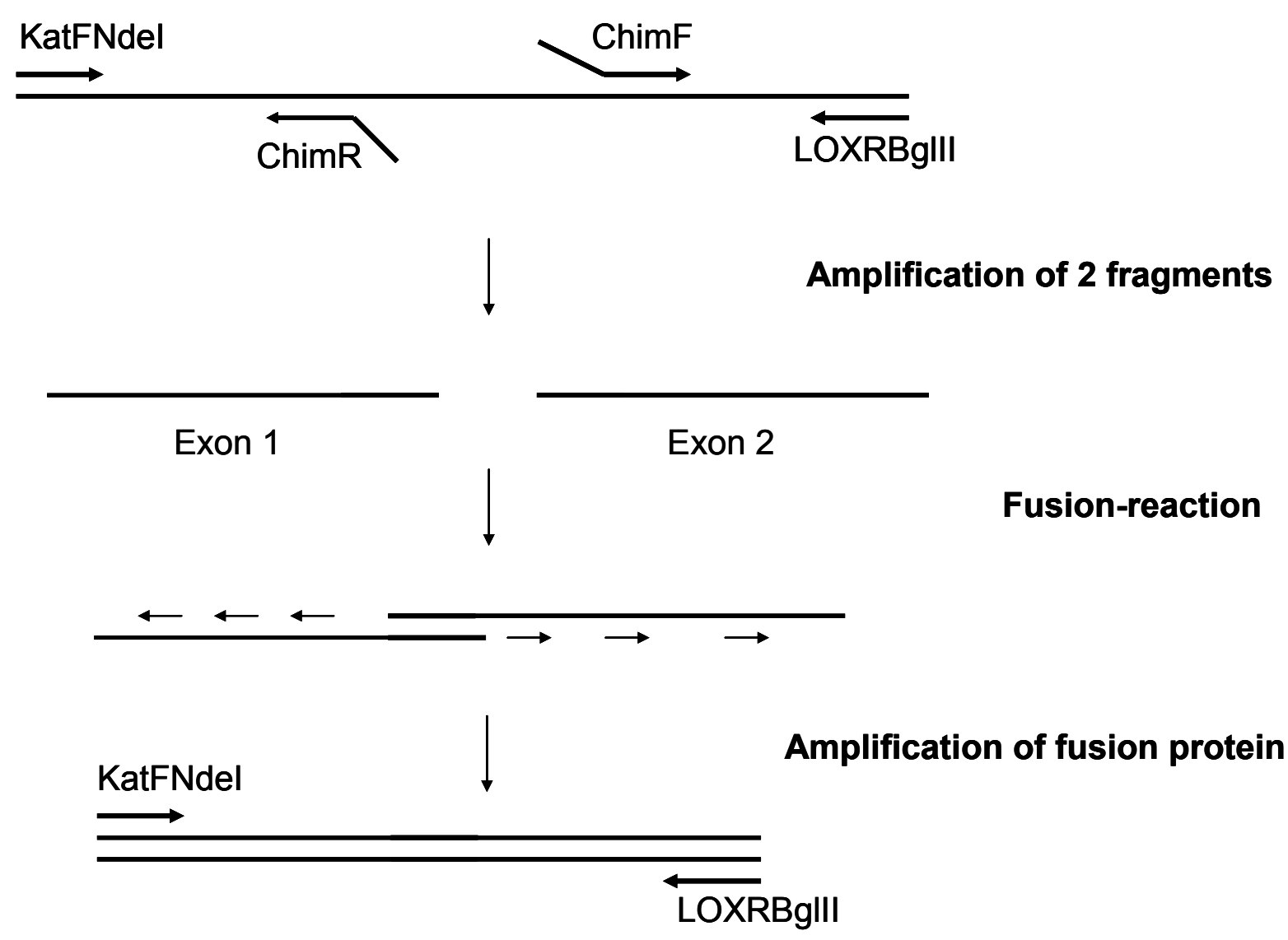

Figure 5. Scheme of the PCR mutagenesis. The reaction was performed in three steps: in the first step the two fragments flanking the transposon are amplified, second the fusion reaction was carried out with the two fragments function as megaprimer and template in parallel. Finally the whole gene without the insertion is amplified using the primer combination KatFNdeI/LOXRBgIII.

Amplification of two fragments was performed with chimeric primers flanking the insertion site and having overlapping ends, which are complement to each other. For amplification of the two fragments (Exon 1 and Exon 2) the same PCR program was used as for the single LOX and Peroxidase domain

The following primers were used:

for exon 1

KatFNdeI and ChimR, 5'-GTAAACGAATCTGGATTGCGTAAGGCTGCCTCCAC'-3 for exon 2 
ChimF 5' GTGGAGGCAGCCTTACGCAATCCAGATTCGTTTAC' -3 and LOXRBglII

The two fragments obtained were mixed together for the fusion reaction. Each fragment functioned as template and megaprimer in parallel. After 12 PCR cycles an aliquot (1:200) of the fused fragments was used as templates for the final amplification with primer KatFNdeI and LOXRBglII using the PCR program for the gene encoding the fusion protein.

\subsection{Gene sequencing}

To confirm the correct orientation and nucleotide sequence of the inserted sequence in the plasmids, sequence analysis was performed. For the sequencing reaction the ABI Prism BigDye Terminator Cycle Sequencing Ready Reaction Kit v1.1(Applied Biosystems) and vector specific primers were used. The Sequence analysis was performed with an ABI PRISM® 3100 Genetic Analyser (Applied Biosystems, Foster, USA).

\subsection{Phylogenetic analysis}

Phylogenetic tree analysis was performed on amino acid sequences of selected LOXs and NpLOX1, NpLOX2 and NspFP using phylip 3.5 (Department of Genome Sciences, University of Washington).

\subsection{Expression of recombinant proteins}

To characterise the activity of the recombinant proteins $(8.3-8.5)$ E. coli expression cells (BL21, Rosetta, HMS174) harbouring the expression construct plasmid were grown in $30 \mathrm{ml}$ LB medium (Bertani 1951) at $37^{\circ} \mathrm{C}$ until they reached $\mathrm{OD}_{600}$ of 0.6. The recombinant protein was induced for $24 \mathrm{~h}$ at $16^{\circ} \mathrm{C}$ by the addition of isopropyl- $\beta-$ thiogalaktopyranosid (0.1 M final concentration). Bacterial cells expressing the His tagged proteins were spinned down $\left(3220 \mathrm{xg}, 20 \mathrm{~min}, 4{ }^{\circ} \mathrm{C}\right)$ and resuspended in $2 \mathrm{ml}$ of $50 \mathrm{mM}$ lysis buffer $\mathrm{pH} 8(50 \mathrm{mM}$ Tris $/ \mathrm{HCl} \mathrm{pH} 8.0,150 \mathrm{mM} \mathrm{NaCl}, 10 \%$ glycerol, $0.1 \%$ Tween 20). The cells were disrupted by $2 \times 20$ s sonification on ice and then centrifuged at $3220 \mathrm{x} g$ for $10 \mathrm{~min}$ at $4{ }^{\circ} \mathrm{C}$. The supernatant and pellet were used for further enzyme assays (SDS-polyacrylamide gelelectrophoresis, Western blot and activity tests). 


\section{Enzyme analysis}

\subsection{SDS Polyacrylamide gelelectrophoresis (SDS-PAGE)}

The separation of proteins was performed with a $10 \%$ denaturating polyacrylamide gel according to a modified method of Laemmli (1970). Gels were assembled using the MiniPROTEAN $^{\circledR} 3$ cell” equipment (Bio-Rad, München). Prior to electrophoresis samples were mixed 1:1 with protein loading buffer $(20 \mathrm{mM} \mathrm{Na-Phosphate} \mathrm{pH} 7.0,20 \%$ (v/v) glycerol, $20 \%(\mathrm{w} / \mathrm{v})$ SDS solution, $0.01 \%$ bromphenolblue solution) and cooked for $5 \mathrm{~min}$ at $100{ }^{\circ} \mathrm{C}$. Between 3 and $5 \mu \mathrm{l}$ of the protein samples were loaded on a 12-lane minigel. As molecular protein marker $5 \mu \mathrm{l}$ of prestained protein marker" (Fermentas, St. Leon-Rot) were used. Gels were run at approximately $100 \mathrm{~V} / 15 \mathrm{~mA}$ until the bromophenolblue band reached the end of the gel.

To visualise proteins, gels were stained with Coomassie Brilliant Blue. Unbound dye was removed by extensive washing of the gel with water.

\begin{tabular}{lrr} 
& Running gel $(10 \%)$ & Stacking gel $(6 \%)$ \\
\hline Acrylamide-Bis $(30 \%)$ & $1650 \mu 1$ & $335 \mu 1$ \\
Buffer & a) $1250 \mu 1$ & b) $625 \mu 1$ \\
water & $2070 \mu 1$ & $1500 \mu 1$ \\
TEMED & $6 \mu 1$ & $5 \mu 1$ \\
$10 \%(\mathrm{w} / \mathrm{v})$ APS & $25 \mu 1$ & $15 \mu 1$ \\
\hline
\end{tabular}

a) Running gel buffer: $1.5 \mathrm{M}$ Tris/HCl, $\mathrm{pH} 8.8,0.4 \%$ (w/v) SDS

b) Stacking gel buffer: $1.0 \mathrm{M}$ Tris/HCl, $\mathrm{pH} 6.8,0.4 \%$ (w/v) SDS

Running buffer, $\mathrm{pH}$ 8.3: $0.05 \mathrm{M}$ Tris, $0.384 \mathrm{M}$ Glycine, 0.1 \% (w/v) SDS

\subsection{Western blot analysis and immunodetection}

The separated proteins were transferred from the SDS-PAGE gel (9.1) to a nitrocellulose membrane (Macherey\&Nagel, Düren) using the Mini Trans-Blot ${ }^{\circledR}$ cell equipment” (Bio$\mathrm{Rad})$. The transfer was performed for $1 \mathrm{~h}$ at $100 \mathrm{~V}$ in the presence of transfer buffer (25 mM Tris, $150 \mathrm{mM}$ Glycine, $10 \%$ (v/v) methanol). Following the transfer nitrocellulose membranes were washed with TBS buffer $(10 \mathrm{mM}$ Tris/ $\mathrm{HCl}, 150 \mathrm{mM} \mathrm{NaCl} \mathrm{pH} 8.0)$ and incubated with a blocking solution $(3 \%(\mathrm{w} / \mathrm{v})$ milk powder in TBS) over night. The next day the membrane was washed with TBS buffer for 2 min to remove the blocking solution. 
In order to detect recombinant proteins expressed with N-terminal His-Tags membranes were incubated for $2 \mathrm{~h}$ at room temperature (RT) with the primary antiserum (mouse antitetra-His, Quiagen, Hilden) diluted to 1:1000 in TBST buffer (TBS buffer with $0.1 \%$ Tween 20). After washing the membrane three times with TBST buffer the secondary antiserum (goat anti mouse, Sigma, München) diluted to 1:1000 in TBST buffer was added and incubated for $1 \mathrm{~h}$ at RT. For visualisation, the second antiserum was conjugated to alkaline phosphatase. Again the membranes were washed three times for $10 \mathrm{~min}$ in TBS buffer and then one washing step with AP-buffer $(100 \mathrm{mM}$ Tris/ $\mathrm{HCl}, 100 \mathrm{mM} \mathrm{NaCl}, 5 \mathrm{mM}$ $\mathrm{MgCl}_{2}, \mathrm{pH}$ 9.5) followed. To visualise the detected proteins, alkaline phosphatase reaction was started by adding $33 \mu 1$ 5-Bromo-4-chloro-3-indolylphosphat and $66 \mu 1$ Nitro blue tetrazolium as described in the manual (Promega, Heidelberg). The reaction was stopped by washing the membrane with water.

\subsection{LOX activity assay}

For the LOX activity test $E$. coli cells expressing the LOX gene were prepared as described in section 8.8. About $500 \mu \mathrm{l}$ of lysate was incubated in $1.5 \mathrm{ml}$ of $50 \mathrm{mM}$ Tris/ $\mathrm{HCl}, \mathrm{pH} 8.0$ with $250 \mu \mathrm{g}$ of FA substrate (LA, ALA, GLA or AA) for $30 \mathrm{~min}$ on ice. The reaction was stopped by adding $40 \mu \mathrm{l}$ glacial acid and $2 \mathrm{ml}$ chloroform. The hydroperoxides were reduced to their corresponding hydroxides by adding $2 \mathrm{ml} 50 \mathrm{mM} \mathrm{SnCl}_{2}$ solved in methanol and the FAs were extracted according to (Bligh and Dyer 1959). The extracted FAs were then analysed by high performance liquid chromatography (HPLC, 10.7).

\subsection{Fusion protein activity assay}

E. coli cells expressing the NspFP gene were prepared as described in section 8.8 . Then $500 \mu \mathrm{l}$ of the supernatant was added to $1.5 \mathrm{ml} 50 \mathrm{mM}$ Tris/HCL, $\mathrm{pH} 8$. By adding $250 \mu \mathrm{g}$ of FAs (LA, ALA, GLA or AA) the reaction was started and then incubated for $30 \mathrm{~min}$ on ice. The reaction was stopped by adding $2 \mathrm{ml}$ diethyl ether and $1 \mathrm{ml}$ water. After acidification to $\mathrm{pH} 3.0$ with glacial acetic acid the samples were centrifuged and the upper phase was used for further analysis. Extract was split and 1/10 of the extract was used for liquid chromatography/mass spectrometry analysis (LC/MS, 10.9) and 9/10 for HPLCanalysis (10.8). Prior GC/MS analysis (10.7) of the dihydroxy FAs, the samples were methylated with trimethylsilyldiazomethane $(0.2 \mathrm{M}$ in hexane, Aldrich). For this the samples were dried under a stream of nitrogen and then solved in $2 \mathrm{ml}$ methanol. $10 \mu \mathrm{l}$ of 
diazomethane was added and the reaction mixture was incubated for $30 \mathrm{~min}$ on a shaker at RT. The reaction was stopped with $0.2 \mu \mathrm{l}$ acetic acid and dried under a stream of nitrogen. For GC/MS analysis the samples were dissolved in $10 \mu 1$ acetonitrile.

The activity of the individual peroxidase-domain was tested with a mixture of unlabelled and radio labelled $\left[1-{ }^{14} \mathrm{C}\right]-9 S$-HPODE having an end concentration of $150 \mathrm{nmol}$ 9-HPODE $\left({ }^{12} \mathrm{C} /{ }^{14} \mathrm{C}\right)\left(150 \mathrm{nmol}\left[1-{ }^{14} \mathrm{C}\right]-(9 S)\right.$-HPODE, $612 \mathrm{kBq}$, kindly provided by Dr. M. Stumpe) and with the product $((9 R)-\mathrm{HPODE})$ of the LOX domain. The reaction was started by mixing $1.5 \mathrm{ml} 50 \mathrm{mM}$ Tris/HCl, $\mathrm{pH} 7$ with $500 \mu 1$ supernatant of the cell lysate and the substrates. After $30 \mathrm{~min}$ incubation on ice the reaction products were extracted according to Bligh and Dyer (1959). The extracts were further analysed by reverse-phase-HPLC (RPHPLC) as described in (Bleé and Joyard 1996).

\subsection{Activity assay in presence of ${ }^{18} \mathrm{O}_{2}$}

For this experiment $50 \mathrm{mM}$ Tris/ $\mathrm{HCl} \mathrm{pH} 8$ was degassed and afterwards the buffer was enriched with inflowed ${ }^{18} \mathrm{O}_{2}$ (Campro Scientific, Berlin). The E. coli expression cultures were prepared according to section 8.8 and the supernatant of the lysate was added to equal volume of buffer and incubated with $250 \mu \mathrm{g}$ LA as substrate for $30 \mathrm{~min}$ on ice. During incubation time the reaction mix was bubbled with ${ }^{18} \mathrm{O}_{2}$.

The reaction was stopped by adding diethyl ether and the extract analysed via reversephase HPLC (10.8) and Gas chromatography/mass spectrometry (GC/MS, 10.7).

\subsection{Activity assay in presence of $\mathrm{H}_{2}{ }^{18} \mathrm{O}$}

E. coli expression cells harbouring the NspFP protein were expressed and harvested via centrifugation at $3220 \mathrm{x} g$ for $20 \mathrm{~min}$ at $4{ }^{\circ} \mathrm{C}$. The cells were resuspended in $500 \mu 1 \mathrm{H}_{2}{ }^{18} \mathrm{O}$ (Chemotrade, Leibzig) and disrupted by $2 \times 20 \mathrm{~s}$ sonification. The crude extract was centrifuged at $3220 \times \mathrm{g}$ for $5 \mathrm{~min}$ at $4{ }^{\circ} \mathrm{C}$ and the supernatant was transferred into a $15 \mathrm{ml}$ tube. The reaction was started by adding $250 \mu \mathrm{g}$ LA. After acidification to $\mathrm{pH} 3.0$ with glacial acid, the dihydroxides were extracted with $2 \mathrm{ml}$ diethyl ether and $2 \mathrm{ml}$ water. The extracted products were subjected to RP-HPLC (10.8) and GC/MS (10.7). 


\subsection{Kinetic analysis of NspFP}

For the determination of putative unstable intermediates, which may be formed during the conversion of FA substrates to dihydroxy FAs products, kinetic studies were performed.

For nine different time points tubes each with $2 \mathrm{ml}$ diethyl ether were prepared and stored on ice. The reaction mix was prepared as described in section 9.4 with $2 \mathrm{ml}$ end volume. The reaction was stopped at the following time points by transferring $200 \mu$ l of the reaction mix to the prepared tubes: $20 \mathrm{~s}, 40 \mathrm{~s}, 1 \mathrm{~min}, 5 \mathrm{~min}, 15 \mathrm{~min}, 30 \mathrm{~min}, 45 \mathrm{~min}, 60 \mathrm{~min}, 90 \mathrm{~min}$ and $120 \mathrm{~min}$. The products were extracted by adding additional $1 \mathrm{ml}$ diethyl ether and $2 \mathrm{ml}$ water and then analysed via RP-HPLC according to 10.7.

\subsection{Determination of LOX- activity in $N$. punctiforme in vivo}

For the measurement of specific LOX activity per total Nostoc protein, a cyanobacteria culture of $100 \mathrm{ml}$ (7.3) was harvested by centrifugation $\left(3220 \mathrm{x}, 10 \mathrm{~min}, 4^{\circ} \mathrm{C}\right)$. The cells were disrupted by adding $1 \mathrm{ml}$ of lysis buffer $\mathrm{pH} 8.0$ (9.3) and sonification for 2 x $30 \mathrm{~s}$ on ice. The cell lysate was centrifuged for at $3220 \mathrm{xg}$ for $5 \mathrm{~min}$ at $4{ }^{\circ} \mathrm{C}$ and the activity of LOX within the cell pellet and supernatant was determined by monitoring 18:2 dependent $\mathrm{O}_{2}$ consumption with an oxygen electrode (Rank Borther, Bottisham, UK). The reaction mixture contained $900 \mu \mathrm{l}$ of $50 \mathrm{mM}$ Tris/ $\mathrm{HCl} \mathrm{pH} 8.0$ and $100 \mu \mathrm{l}$ of either supernatant or pellet in a total volume of $1 \mathrm{ml}$. The reaction was started by adding $10 \mu 1$ of $10 \mathrm{mM}$ sodium linoleate. Protein content was determined by the method of (Bradford 1976).

\subsection{Determination of dihydroxy fatty acids in vivo}

In order to test whether the cyanobacterial strains SAG 25.82, PCC7120A and PCC7120P produce dihydroxy FA in vivo the following experiment was performed.

Three $100 \mathrm{ml}$ cultures of each cyanobacterial strain (PCC7120A, PCC7120P and SAG 25.82) were harvested $\left(3220 \mathrm{~g}, 4^{\circ} \mathrm{C}, 5 \mathrm{~min}\right)$ and the pellet resuspended with $1 \mathrm{ml} 50 \mathrm{mM}$ Tris/ $\mathrm{HCl} \mathrm{pH}$ 8.0. Two samples of each strain were sonified for $40 \mathrm{~s}$ on ice. Then to one sonified sample $250 \mu \mathrm{g}$ LA or $5.5 \mathrm{MBq}$ of radio labelled $\left[1-{ }^{14} \mathrm{C}\right]$-LA was added. All three samples of each strain were then incubated for $30 \mathrm{~min}$ at RT. The incubation was stopped by adding $5 \mathrm{ml}$ of diethyl ether. Further on the formed oxylipins were analysed via RPHPLC (10.8) and GC/MS (10.7) 


\section{Lipid analysis}

\subsection{Lipid extraction and thin layer chromatography (TLC)}

Algal cells were harvested in a $10 \mathrm{ml}$ glass tube by centrifugation. Lipids were extracted from the pellet with chloroform/methanol 1:2(v/v) for $4 \mathrm{~h}$ at $4{ }^{\circ} \mathrm{C}$ according to (Folch et al. 1957). The samples were centrifuged ( $\left.3220 \times \mathrm{g}, 5 \mathrm{~min}, 4{ }^{\circ} \mathrm{C}\right)$ and the supernatant was collected into a new glass tube, whereas the pellet was reextracted with chloroform/methanol 2:1 (v/v) over night at $4{ }^{\circ} \mathrm{C}$. The resulting lipid extracts were combined and filtered through cotton wool soaked with $\mathrm{NaSO}_{4}$ to remove moisture. After evaporating the solvent under nitrogen, samples were fractionated into neutral lipids, glycolipids and phospholipids on $1 \mathrm{ml}$ silica Bond Elut columns (Varian, Darmstadt). Neutral lipids were eluted with $10 \mathrm{ml}$ chloroform, glycolipids with $10 \mathrm{ml}$ acetone/isopropanol 9:1 (v/v) and finally the phospholipids with $10 \mathrm{ml}$ methanol. These three fractions were further resolved on $20 \mathrm{~cm}$ x $20 \mathrm{~cm}$ silica gel 60 TLC plates (Merck, Darmstadt).

Neutral lipids were developed with chloroform/acetone 96:4 (v/v), glycolipids with chloroform/methanol 85:15 (v/v) and phospholipids with chloroform/methanol/acetic acid $65: 25: 8(\mathrm{v} / \mathrm{v} / \mathrm{v})$.

Individual lipid classes were identified according to co-migration with authentic standards after incubation in $\mathrm{CuSO}_{4}$ solution $\left(10 \mathrm{~g} \mathrm{CuSO}_{4} \times 5 \mathrm{H}_{2} \mathrm{O} / 92 \mathrm{ml} \mathrm{H} \mathrm{H}_{2} \mathrm{O}+8 \mathrm{ml} \mathrm{H}_{3} \mathrm{PO}_{4}\right)$ and heating at $180^{\circ} \mathrm{C}$. In addition phospholipids, including betaine lipids, were stained with Dragendorff reagent (Fluka, München) and phosphatide reagent (Solution I/Solution II/ $\mathrm{H}_{2} \mathrm{O}$ 1:1:2 (v/v/v), Solution I: $40.1 \mathrm{~g}$ molybdene oxide in $1125 \mathrm{~N} \mathrm{H}_{2} \mathrm{SO}_{4}$, Solution II: $1.78 \mathrm{~g}$ molybdene in $500 \mathrm{ml}$ solution I). For analysis of the lipid bound FAs, the lipids within were separated by TLC and visualised by $0.2 \% 8$-anilino-1-naphthalene-sulfonic acid (ANS) under UV-light $(\lambda=365 \mathrm{~nm}$ and $254 \mathrm{~nm}$ ). The visualised lipids were isolated by scraping the silica powder and directly used for transmethylation (10.3) and gas chromatography (GC) analysis (see 10.6).

\subsection{Alkaline hydrolysis, transesterification and extraction of FAMEs}

For the analysis of total FA profiles of different microalgal strains (7.1), the dry weight of lyophilised alga material was determined and then the samples were transferred to a $2 \mathrm{ml}$ tube. The samples were extracted by adding $405 \mu 1$ of methanol/toluol 2:1 (v/v) followed 
by homogenisation the cells with a potter (Heidolph RZR 2020, Schwabach) for $30 \mathrm{~s}$. To avoid autoxidation, the samples were overlaid with argon. As internal standard $10 \mu \mathrm{g}$ of tripentadecanoate (diluted in $10 \mu \mathrm{l}$ toluol) were added. Transesterification of lipid bound FAs to their corresponding FAMEs was accomplished by adding $150 \mu 1$ sodium methoxide (Hornung et al. 2002). After 20 min shaking at room temperature the FAMEs were extracted two times with $500 \mu \mathrm{ln}$-hexane and $500 \mu 11 \mathrm{M} \mathrm{NaCl}$. The hexane phases were transferred into a $1.5 \mathrm{ml}$ tube and dried under streaming nitrogen. Finally FAMEs were redissolved in $10 \mu 1$ acetonitrile and analysed by GC (10.6).

\subsection{Acidic hydrolysis, methylation of FA carboxyl groups and extraction of FAMEs}

For acidic hydrolysis, $2 \mathrm{ml}$ of FAME-solution containing $2.75 \%$ (v/v) $\mathrm{H}_{2} \mathrm{SO}_{4}(95-97 \%)$ and $2 \%(\mathrm{v} / \mathrm{v})$ dimethoxypropan in methanol was either added to algal cell pellet (7.1) or to the silica powder resulting from TLC (10.1 and 10.4). The sample was incubated for $1 \mathrm{~h}$ at $80^{\circ} \mathrm{C}$ and after adding $200 \mu \mathrm{l} 5 \mathrm{M} \mathrm{NaCl}$ the FAMEs were extracted two times with $2 \mathrm{ml}$ hexane. The hexane phase was dried under streaming nitrogen and redissolved with equal volumes of water and hexane. The hexane phase was filtered through cotton wool soaked with $\mathrm{NaSO}_{4}$ and dried again under streaming nitrogen. Finally the sample was redissolved in $10 \mu \mathrm{l}$ acetonitrile for GC (10.6) and GC/MS (10.7) analysis or in $80 \mu \mathrm{l}$ methanol/water/acetic acid 75:25:0.1 (v/v/v) for isolation via RP-HPLC (10.8).

\subsection{Preparation of 4,4-dimethyloxaline (DMOX) derivatives}

The positions of the double bonds of unknown FAME isomers were determined by preparing the corresponding DMOX derivatives often allowing mass spectrometric identification (Fay and Richli 1991).

FAMEs were prepared as described in 10.3, but the hexane phases were transferred into a $1.5 \mathrm{ml}$ glass tube. Samples were dried under streaming nitrogen and $200 \mu 1$ 2-alkenyl-4,4dimethyloxazoline (Sigma, München) were added. After incubation at $180{ }^{\circ} \mathrm{C}$ over night in a heating block, samples were cooled to RT and transferred with $2 \mathrm{ml}$ dichloromethane into a $12 \mathrm{ml}$ glass tube and reextracted with $5 \mathrm{ml}$ hexane and $2 \mathrm{ml}$ water. The hexane phase was dried under streaming nitrogen and redissolved with $50 \mu 1$ chloroform. The DMOX derivatives were separated on a $20 \mathrm{~cm}$ x $20 \mathrm{~cm}$ silica gel 60 TLC plate (Merck) with petrol ether/diethyl ether $2: 1(\mathrm{v} / \mathrm{v})$ as a developing solvent. The plate was sprayed with $0.2 \%$ ANS to visualize the DMOX derivatives under UV-light. The blue/yellow band of the 
DMOX derivatives was scraped out and the derivatives extracted by consecutive addition of $0,4 \mathrm{ml}$ water, $2 \mathrm{ml}$ methanol, $2 \mathrm{ml}$ chloroform and $2 \mathrm{ml}$ saturated $\mathrm{NaCl}$ solution. Between each step the sample was vigorously mixed and finally centrifuged for $5 \mathrm{~min}$ at $3220 \mathrm{x} g$ to separate phases. The lower phase was transferred into a new glass tube and dried under nitrogen stream, redissolved in $10 \mu \mathrm{l}$ acetonitrile and analysed with GC/MS (10.7).

\subsection{Preparation of picolinyl ester}

For the determination of the double bond positions of 18:5n-3, picolinyl ester were prepared according to Destaillats and Angers (2002). A solution of potassium tert-butoxide in tetrahydrofuran $(0.1 \mathrm{ml}, 1.0 \mathrm{M})$ was added to $0.2 \mathrm{ml} 3$-pyridylcarbinol. After mixing, the 18:5n-3 sample in $1 \mathrm{ml}$ dry dichloromethane was added, and the mixture was incubated at $40^{\circ} \mathrm{C}$ for $30 \mathrm{~min}$ in a closed vial. After cooling to RT, $2 \mathrm{ml}$ water and $4 \mathrm{ml}$ hexane was added, and the organic phase is collected, dried over anhydrous sodium sulfate, and evaporated. The sample is dissolved in isohexane for GC-MS analysis (10.7).

\subsection{Identification of FAMEs by gas chromatography (GC) with flame ionisation detection}

The prepared FAMEs were analysed by GC coupled to flame ionisation detector using a capillary DB-23 column $(30 \mathrm{~m} \times 0.25 \mathrm{~mm}, 0.25 \mu \mathrm{m}$ coating thickness, J\&W, Scientific, Agilent, Waldbronn) according to Hornung et al (2002). Helium was used as carrier gas with a flow of $0.1 \mathrm{ml} / \mathrm{min}$. The temperature gradient was $150{ }^{\circ} \mathrm{C}$ for $1 \mathrm{~min}, 150-200{ }^{\circ} \mathrm{C}$ at $4 \mathrm{~K} / \mathrm{min}, 200-250^{\circ} \mathrm{C}$ at $5 \mathrm{~K} / \mathrm{min}$ and $250^{\circ} \mathrm{C}$ for $6 \mathrm{~min}$. As retention time standard a FAME mixture F.A.M.E. Mix, C4-C24" (Sigma, München) was injected before every $50^{\text {th }}$ run. The injection volumes depended on the concentration of FAMEs within in the sample.

\subsection{Identification of FAMEs by GC/MS}

FID signals which were not identified by GC and either represent FAMEs or other unpolar substances were further analysed by their mass spectra using a 6890 Gas Chromatograph/5973 Mass Selective Detector system (Agilent, Waldbronn). The GC/MS conditions were the same as for GC-Analysis (10.6). Electron energy of $70 \mathrm{eV}$, an ion source temperature of $230{ }^{\circ} \mathrm{C}$, and a temperature of $260{ }^{\circ} \mathrm{C}$ for the transfer line were used The identification of unknown substances was done by comparison of the obtained mass 
spectra with the mass spectra library NIST98 and the Lipid Library" of the Scottish Crop Science research Institute http://www.lipidlibrary.co.uk/index.html.

\subsection{Identification and preparation of FAs and oxylipins by high performance liquid chromatography (HPLC)}

HPLC analysis was performed with an Agilent 1100 HPLC system coupled to a diode array detector.

Reverse phase HPLC (RP-HPLC) analysis were carried out using a EC250/2 Nucleosil 120-5 C18 column (250 x $2.1 \mathrm{~mm}, 5 \mu \mathrm{m}$ particle size, Macherey\&Nagel, Düren). The absorbance at $234 \mathrm{~nm}$ (conjugated diene system of the hydroxy FAs) was recorded simultaneously during all chromatographic steps.

Reaction products of the LOX-activity assay (9.3) were separated from the used FA substrates by using 90:10:0.1 methanol:water:acetic acid $(\mathrm{v} / \mathrm{v} / \mathrm{v})$ as solvent and a flow rate of $\quad 0.2 \mathrm{ml} / \mathrm{min}$. As standards $(9 Z, 11 E, 13 S)-13$-hydroxy-9,11-octadecadienoic acid (13-HODE), LA, 13-HOTE or ALA were used.

For the separation of the oxylipins JA, dinor-oPDA, oPDA, C16-and C18monohydroxylated FAs (10.8) the following solvents were combined: A: 75:25:0.1 methanol:water:acetic acid (v/v/v) and solvent B: 100:0:0.1 methanol:water:acetic acid $(\mathrm{v} / \mathrm{v} / \mathrm{v})$. The gradient elution profile was as follows starting with a flow rate of $0.18 \mathrm{ml} / \mathrm{min}: 0-10 \mathrm{~min}, 80 \%$ A $20 \% \mathrm{~B} ; 10-15 \mathrm{~min}, 80 \%$ A $20 \% \mathrm{~B}$ to $100 \% \mathrm{~B} ; 15-$ $17 \mathrm{~min}$ flow increases from $0,18 \mathrm{ml} / \mathrm{min}$ to $0,36 \mathrm{ml} / \mathrm{min} ; 17-25 \mathrm{~min} 100 \% \mathrm{~B} ; 25-27 \mathrm{~min}$ $100 \%$ B to $20 \%$ B $80 \%$ A. Oxylipins were separated by collecting the respective fractions. The following standards were used to determine the retention time of the different oxylipin classes: JA, dinor-oPDA, (7Z,9E,11S,13Z)-11-hydroxy-7,9,13hexadecatrienoic acid (11HHT), oPDA, 13-HOTE and 13-HODE.

Dihydroxy FAs produced by the fusion protein reaction (9.4) were separated from monohydroxy FAs by RP-HPLC eluted with a combination of the following solvents: A: 50:50:0.1 acetonitrile:water:acetic acid (v/v/v) and B: 100:0.1 acetonitrile:acetic acid (v/v). The gradient elution profile was as follows starting with a flow rate of $0.18 \mathrm{ml} / \mathrm{min}$ : 0 $10 \mathrm{~min}, 100 \% \mathrm{~A} ; 10-20 \mathrm{~min} 100 \% \mathrm{~A}$ to $0 \% \mathrm{~A} 100 \% \mathrm{~B} ; 20-22 \mathrm{~min}$ flow rate increasing from $0.18 \mathrm{ml} / \mathrm{min}$ to $0.36 \mathrm{ml} / \mathrm{min} ; 22-27 \mathrm{~min} 100 \% \mathrm{~B} ; 27-32 \mathrm{~min} 100 \% \mathrm{~B}$ to $100 \% \mathrm{~A}$, $32 \min -35 \min 100 \% \mathrm{~A}$. 
Straight phase HPLC (SP-HPLC) of hydroxy FA isomers was carried out on a Zorbax SIL column ( $150 \times 2.1 \mathrm{~mm}, 5 \mu \mathrm{m}$ particle size, Agilent, Waldbronn) running with a solvent system of n-hexane:2-propanol:acetic acid 100:1:0.1 (v/v/v) at a flow rate of $0.2 \mathrm{ml} / \mathrm{min}$. As retention time standard an extract from leaves of stinging nettles supplemented with 13 $\gamma$-HOTE, (9Z,11E)-13-keto-9,11-octadecadienoic acid (13-KODE), (10E,12Z,15Z)-9keto-10,12,15-octadecatrienoic acid (9-KOTE) was injected.

Chiral phase HPLC (CP-HPLC) allows the separation of enantiomeric compounds due to chiral molecules which are bound to the stationary phase (. CP-HPLC of the hydroxy FAs was carried out on a Chiralcel OD-H column $(150 \mathrm{~mm}$ x $2.1,5 \mu \mathrm{m}$ particle size, Diacel, supplied by VWR, Darmstadt) with a solvent system of n-hexane:2-propanol:acetic acid 100:5:0.1 (v/v/v) and a flow rate of $0.1 \mathrm{ml} / \mathrm{min}$.

\subsection{Analysis of NspFP products by HPLC/MS analysis}

The analysis was done by a reverse-phase HPLC/MS Surveyor HPLC system (Thermo Finnigan, Darmstadt) equipped with a photo diode array detector coupled to a LCQ Advantage electrospray ionisation ion trap mass spectrometer (Thermo Finnigan, Darmstadt). The fusion protein products were separated by a EC 250/2 Nucleodure 100-5 C18ec column (250 x $2.1 \mathrm{~mm}, 5 \mu \mathrm{m}$ particle size, Macherey\&Nagel, Düren). Solvents and also the gradient were the same as for RP-HPLC (10.8). The mass spectrometer was operated in negative mode with the source voltage set to $4 \mathrm{kV}$, and a capillary voltage of $27 \mathrm{~V}$ and $300{ }^{\circ} \mathrm{C}$. In full mass spectrometry (MS) mode, scans were collected between $\mathrm{m} / \mathrm{z}$ values of 50 and 400 (Hughes et al. 2006).

\subsection{Nucleic magnet resonance (NMR) analysis}

Nucleic magnet resonance analysis is a technique for structural analysis and quantification broad range of molecules, including FAs and their derivatives (Vollhardt and Schore 1995). The most commonly measured nuclei are hydrogen-1 and carbon-13. With NMR the magnetic nuclei are studied by aligning them with a powerful external magnetic field and perturbing this alignment using an electromagnetic field. The resulting response to the external perturbing electromagnetic field is the phenomenon that is exploited in NMR spectroscopy and magnetic resonance imaging (Hart 1989). 
About $50 \mu \mathrm{g}$ of FA was used for NMR analysis. NMR analysis was performed in collaboration with Dr. Andrea Porzel (Leibniz-Institute of Plant Biochemistry in Halle/Saale). The 1D- and 2D-NMR-spektra were analysed with a Varian Inova500Spektrometer at $499.799 \mathrm{MHz}\left({ }^{1} \mathrm{H}\right)$ using a NALORAC 3-mm microsample inverse detection probe. As solvent, $\mathrm{CDCl}_{3}$ was used. The chemical shifts of ${ }^{1} \mathrm{H}$ - referred to internal trimethylsilyl (TMS) $\left(\delta{ }^{1} \mathrm{H}=0 \mathrm{ppm}\right)$, and the chemical shifts of ${ }^{13} \mathrm{C}$-referred to internal $\mathrm{CDCl} 3\left({ }^{13} \mathrm{C}=77.0 \mathrm{ppm}\right)$.

\subsection{Purification of methylated 18:5n-3}

The extracted FAMEs (see 10.3) of the two Prymnesium strains (SAG 18.97 and SAG 127.79) were separated with RP-HPLC using an EC 250/2 Nucleosil 120-5 C18 column $(250 \times 2.1 \mathrm{~mm}, 5 \mu \mathrm{m}$ particle size, Macherey\&Nagel, Düren) and using the following solvents: A 75:25:0.1 (v/v/v) methanol:water:acetic acid and B 100:0:0.1 (v/v/v) methanol:water:acetic acid. The gradient elution profile starting with a flow of $0.18 \mathrm{ml} / \mathrm{min}$ was as follows: $0-15 \mathrm{~min}, 80 \%$ A $20 \% \mathrm{~B} ; 15-17 \min 80 \% \mathrm{~A} 20 \% \mathrm{~B}$ to $100 \% \mathrm{~B}$ and flow from 0.18 to $0.36 \mathrm{ml} / \mathrm{min} ; 17-25 \mathrm{~min}, 100 \% \mathrm{~B} ; 25-27 \mathrm{~min}, 100 \% \mathrm{~B}$ to $80 \%$ A $20 \%$ B; 27-30 min $80 \%$ A $20 \%$ B.

Fractions containing FAMEs were collected into a glass vial and dried under streaming nitrogen. The purity of collected FAMEs was tested by GC/MS as described in section 10.6. The concentration of $18: 5 \omega 3$ was determined by co injection of methylated LA (meLA, Sigma, München).

\subsection{Oxylipin profiling of SAG microalgal strains}

The profiling of oxylipins from the SAG microalgal strains was performed with the FAME extracts obtained by alkaline hydrolysis (10.2), which were already used for the FAME screen. For oxylipin analysis carbonyl groups of isolated ketols were reacted with methylmethoxyamine hydrochloride reagent (MOX ${ }^{\mathrm{TM}}$ Fluka, Seelze) resulting in the formation of methoxime derivatives (Horning et al. 1968) and hydroxy groups were converted to TMS groups with N,O-bis-(trimethylsilyl)-trifluoroacetamide (BSTFA, Fluka, Seelze). For derivatisation, the FAME extracts were transferred into glass tubes and dried under streaming nitrogen. After adding $50 \mu \mathrm{l}$ of a $2 \%$ solution of methoxyamine hydrochloride in pyridine the samples were incubated over night at RT. Oxylipins were 


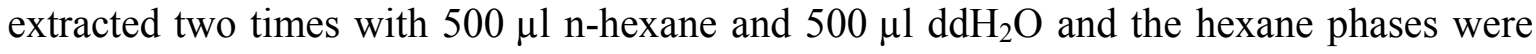
transferred into a $1.5 \mathrm{ml}$ tube and dried under streaming nitrogen. Finally the oxylipins were redissolved in $4 \mu \mathrm{l}$ acetonitrile and $1 \mu \mathrm{l}$ BSTFA. The silylated and methoxylated products were used directly for GC-MS analysis (10.7).

\subsection{Oxylipin profiling of the cyanobacteria Nostoc punctiforme and Nostoc sp.}

Endogenous LOX product formation in crude extracts of cyanobacteria was determined for the cyanobacterial strains according to Stumpe et al (2005). Two algae cultures of $100 \mathrm{ml}$ (7.1) were harvested by centrifugation $\left(3220 \mathrm{xg}, 10 \mathrm{~min}, 4^{\circ} \mathrm{C}\right)$ and all pellets were resuspended in $1 \mathrm{ml} \mathrm{BG11} \mathrm{medium.} \mathrm{One} \mathrm{culture} \mathrm{was} \mathrm{wounded} \mathrm{by} \mathrm{sonification} \mathrm{for} 40 \mathrm{~s}$ on ice (about $20 \%$ of cells were lysed by this treatment). Cultures were incubated for $30 \mathrm{~min}$ at RT to allow oxylipin production. Samples were stored at $-20{ }^{\circ} \mathrm{C}$.

For analysis of hydroxyl FAs and hydroperoxy FAs to about 0.5 to $1.5 \mathrm{~g}$ fresh weight of frozen algal cells $15 \mathrm{ml}$ of extraction medium (n-hexane:2-propanol, 3:2 (v/v) with $0.0025 \%(\mathrm{w} / \mathrm{v})$ butylated hydroxytoluene) and $500 \mathrm{mg}$ glass pearls $(0.25 \mathrm{~mm}-0.5 \mathrm{~mm})$ were added. As internal standards, $(6 Z, 9 Z, 11 E, 13 S)$-13-hydroxy-6,9,11-octadecatrienoic

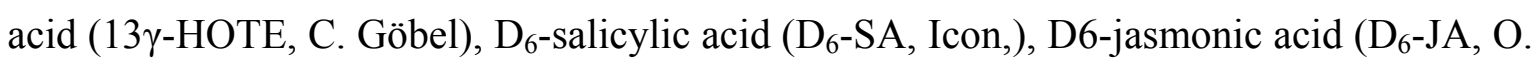
Miersch, Halle/Saale) and $\mathrm{D}_{5}$-12-oxo-phytodienoic acid ( $\mathrm{D}_{5}$-oPDA, O. Miersch) was added each at final concentration of $100 \mathrm{ng}$. The extract was homogenized by shaking for $10 \mathrm{~min}$ at $4{ }^{\circ} \mathrm{C}$ and then centrifuged at $3220 \mathrm{xg}$ for $10 \mathrm{~min}$ at $4{ }^{\circ} \mathrm{C}$. The clear upper phase was collected and a $6.7 \%(\mathrm{w} / \mathrm{v})$ solution of potassium sulfate was added until the total volume reached $47 \mathrm{ml}$. After vigorous shaking and centrifugation $\left(3220 \mathrm{xg}, 10 \mathrm{~min}, 4^{\circ} \mathrm{C}\right)$ the upper hexane rich layer containing oxylipins was dried under streaming nitrogen and redissolved in $80 \mu \mathrm{l}$ of methanol:water:acetic acid 85:15:0.1 (v/v/v). Oxylipins were purified on RP-HPLC, then SP-HPLC was applied to separate the hydro(pero)xy derivatives of LA and ALA according to 10.8 .

For the analysis of esterified oxylipins and FAs, triricinoleate and triheptadecanoate were used as internal standards and the extraction was performed as described above. After removing of the solvent, $405 \mu 1$ toluol:methanol 1:2 (v/v) and $150 \mu 1$ of $0.5 \mathrm{mM}$ sodium methoxide (Sigma, München) were added. After incubation for $20 \mathrm{~min}$ at RT, $0.5 \mathrm{ml}$ of $1 \mathrm{M} \mathrm{NaCl}$ and $50 \mu \mathrm{l}$ of $\mathrm{HCl}(37 \%, \mathrm{v} / \mathrm{v})$ were added, and the resulting FAMEs and their oxidized derivatives were extracted twice each with $0.75 \mathrm{ml}$ of hexane. The combined organic phases were evaporated to dryness under streaming nitrogen and dissolved in $200 \mu 1$ of methanol. An aliquot of $20 \mu 1$ of this solution was dried under streaming nitrogen 
and dissolved in $10 \mu \mathrm{l}$ of acetonitrile for the analysis of the FA methyl esters by GC (10.6). The oxylipin methyl esters were analysed by HPLC according to the analysis of the free oxylipins. Methyl esters of jasmonic acid and 12-oxo phytodienoic acid were detected by gas chromatography/electron impact mass spectrometry (GC/MS, 10.7). For derivatisation, $1 \mu 1$ of BSTFA solution was added to the sample. For quantification, the ions $m / z 224$ (JA), 238 (oPDA) and 210 (dinor-oPDA) were used, respectively.

\subsection{Feeding experiment with labelled fatty acid substrates}

To study the biosynthetic pathway in vivo, P. parvum SAG 127.79 was cultured in $100 \mathrm{ml}$ 1/2SWES medium at $20^{\circ} \mathrm{C}$ until the $\mathrm{OD}_{600}$ reached 0.6-0.8 (7.2). The culture was then split into three cultures of $30 \mathrm{ml}$.

To investigate whether the algal cells take up external added FAs, two cultures were 'fed' with $5.5 \mathrm{MBq}\left[1-{ }^{14} \mathrm{C}\right]$-linoleic acid (Amersham). The third culture without added LA was used as negative control. After two days of incubation (usual culture conditions, 7.2) the three cultures were harvested $\left(2500 \mathrm{x} \mathrm{g}, 5 \mathrm{~min}, 4^{\circ} \mathrm{C}\right)$, the pellet was washed several times with water and then FAs were extracted via acidic hydrolysis (10.3). The radio labelled samples were analysed by Radio-RP-HPLC using the same gradient as for the purification of 18:5n-3 (10.11).

Further feeding experiments were performed with $1 \mathrm{mg}\left[\mathrm{U}-{ }^{13} \mathrm{C}\right]-\mathrm{LA}$ (Larodan, Malmö, Sweden), [U- $\left.{ }^{13} \mathrm{C}\right]-A L A$ (Sigma, München) and [19,19,20,20,20-D 5 -EPA (Larodan, Malmö, Sweden). In this case the extracted FAMEs (10.3) were analysed by GC/MS (10.7). 


\section{Results}

\section{Large scale profiling of lipid bound fatty acids}

According to the current knowledge no systematic analysis has been published on a large scale basis on the profiles of lipid bound FAs found in microalgae. In order to exploit the biocapacities of microalgae to full extent, one aim of this work was the characterisation of FA profiles from a broad range of microalgae. For this purpose the culture collection of microalgal strains at the SAG was used and all available SAG algal strains were analysed for their lipid bound FAs.

\subsection{A database of profiles for diverse microalgae}

The characterisation of FA profiles of the SAG microalgal strains was performed by screening medium, long and very long chain FAs (C14-C22) esterified within lipids.

The algal cultures were harvested in the stationary phase, which was between 3 months and one year (7.1). The lipids (FAs) were extracted from lyophilised samples (10.2) and isolated FAMEs were analysed via GC (10.6). Tripentadecanoate was added to each sample for quantification and the FAMEs were identified according to the retention time of the corresponding peaks in the standard F.A.M.E. Mix C4-C24" (Fig. 6). FAMEs or other unpolar substances which were not identified due to their retention time were analysed via GC/MS (10.7) to generate their mass spectra. As references the MS-Nist98 library and the Wiley-library were used. 
A

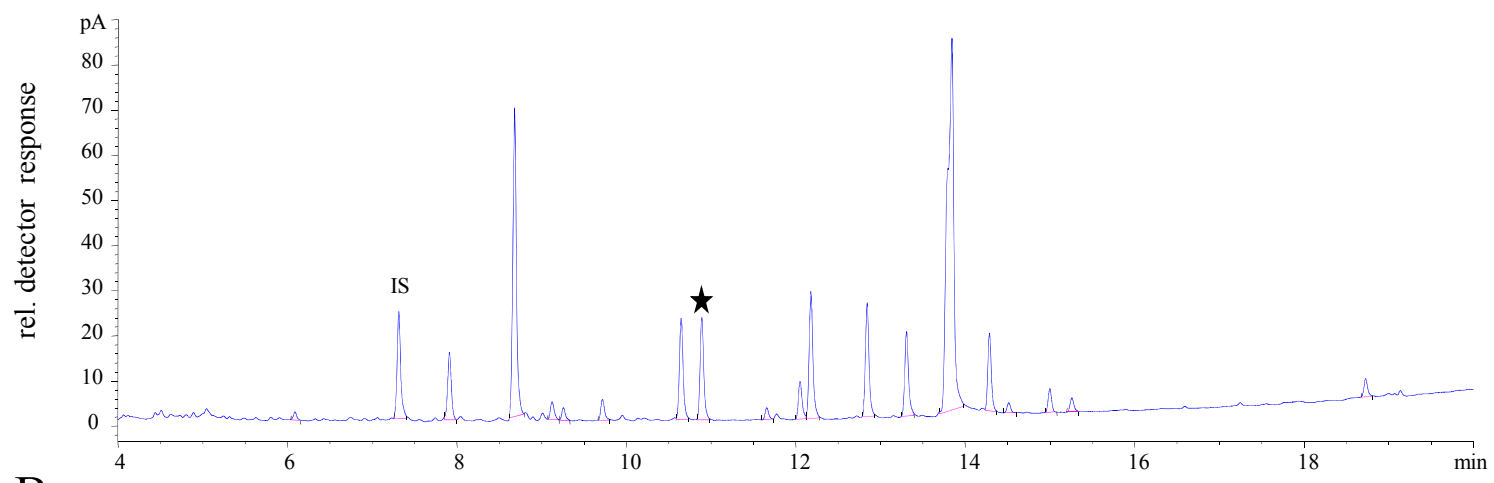

B

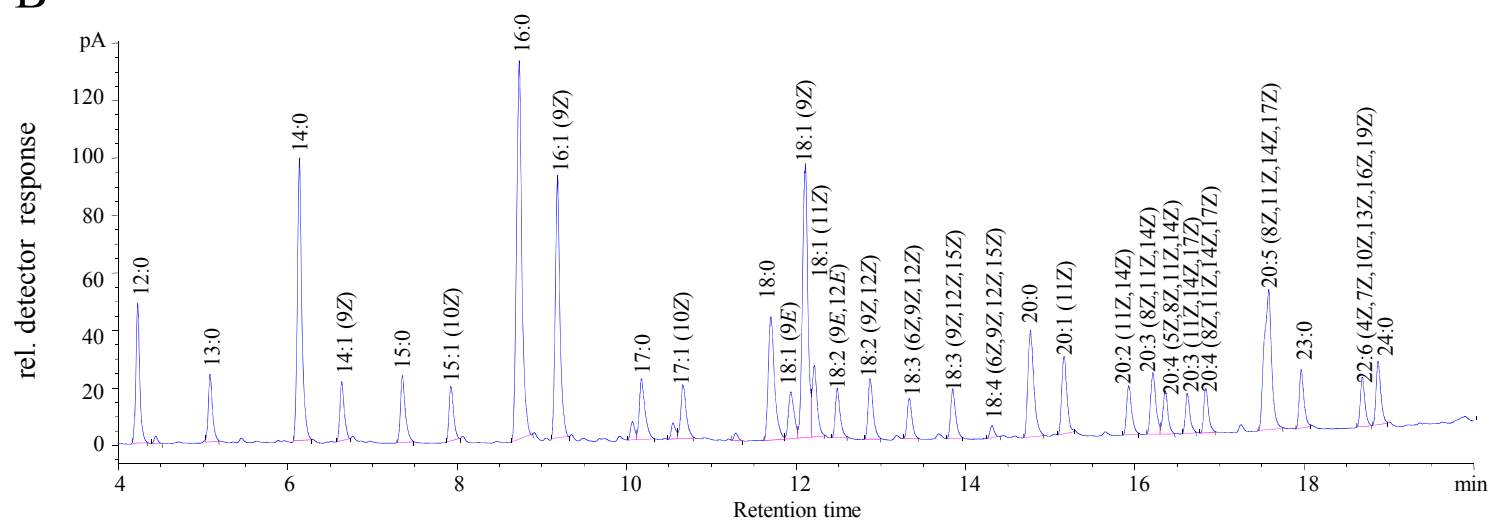

Figure 6. GC chromatogram of Chlamydomonas sp and the standard F.A.M.E. Mix". A, Chlamydomonas sp. Hg1973-49 and B, standard "F.A.M.E.-Mix". Peak indicated with a star represent unidentified substance. Prior to extraction tripentadecanoate was added as internal standard (IS).

Signals which were not identified by these libraries but contained the FAME specific McLafferty $(m / z=74)$ fragments were further analysed by preparing the corresponding DMOX derivatives (10.4). The mass spectra of DMOX derivatives of FAs tend to be distinct and permit the location of double bonds, when they are located in central positions (Zhang et al. 1988). As an example the mass spectrum of the DMOX derivative of $16: 3^{(7 Z, 10 Z, 13 Z)}$ is illustrated in Fig. 7. Double bonds in central positions ( $>$ C6) are separated by the appropriate gaps of $12 \mathrm{amu}$ (marked by stars in Fig. 7). Double bonds at the position 5 and 4 can only be deduced from the characteristic fingerprint ion at $\mathrm{m} / \mathrm{z}=153$ and $\mathrm{m} / \mathrm{z}=$ 152 , respectively. 
$16: 3(7 Z, 10 Z, 13 Z)$

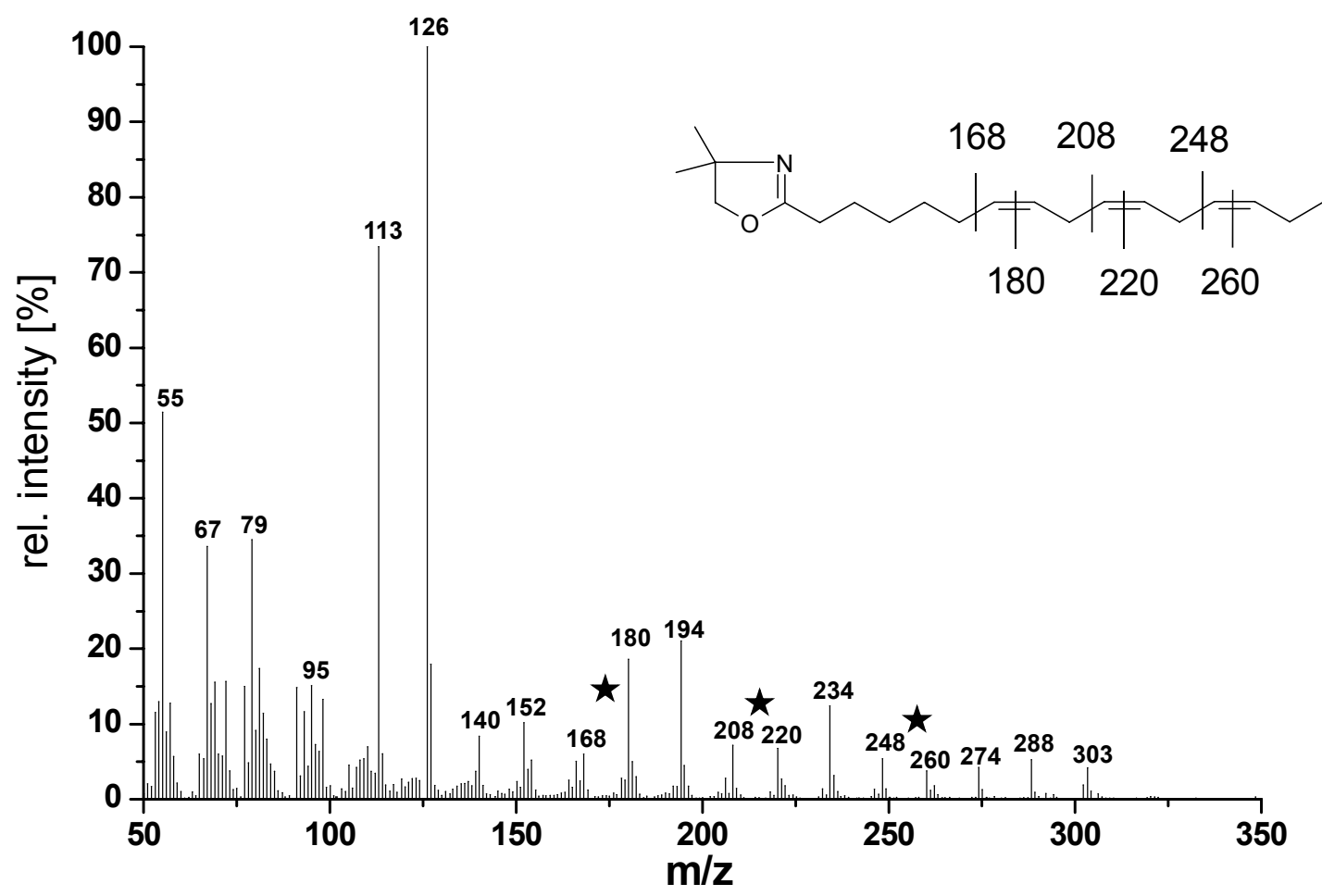

Figure 7. Mass spectrum of the DMOX derivative of $16: 3^{(7 Z, 10 Z, 13 Z)}$. The double bonds in positions 7, 10 and 13 are identified by the gaps of 12 amu between $\mathrm{m} / \mathrm{z} 168$ and 180, $\mathrm{m} / \mathrm{z} 208$ and 220 and between $\mathrm{m} / \mathrm{z} 248$ and 260. They are indicated by a star.

In total 2347 SAG microalgae strains were screened for their FA profile and all chromatograms of these samples have been analysed by using the ChemStation software version 9.03 (Agilent, Waldbronn). For this all peaks spanning a peak area of more than 50 units were integrated. The amount of each FAME was calculated using a defined amount $(1 \mu \mathrm{g})$ of the internal standard tripentadecanoate and the dry weight (d.w.) of each sample: area of peak x $1 \mu \mathrm{g} /$ area of tripentadecanoate $/ \mathrm{mg} \mathrm{d} . \mathrm{w}=\mu \mathrm{g}$ FAME $/ \mathrm{mg}$ d.w.

A database has been established, which contains all FAMEs identified and some other metabolites given in $\mu \mathrm{g}$ FAME/mg d.w. as well as in percent per total FA. Fig. 8 shows an example for data included in the database which uses the Microsoft Excel format. For each sample analysed, the number of sample and SAG strain number is given as well as the species name and the amount of each substance identified. 


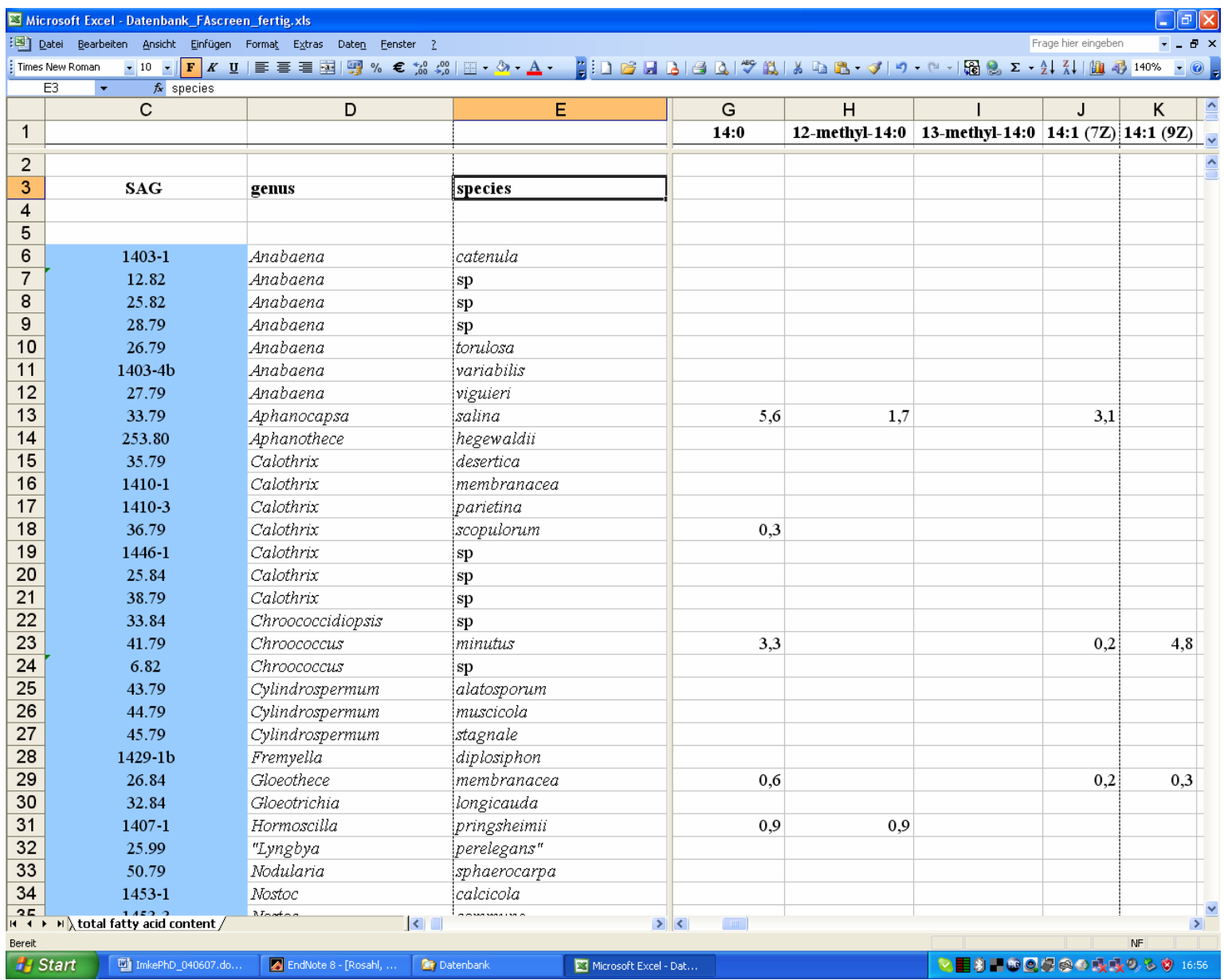

Figure 8. Example of data compiled in the FAME database established of all SAG microalgae strains screened. The database contains information about SAG strain number (column C), species name (column D\&E) and the FAME content of each strain in $\mu \mathrm{g}$ FAME/mg d.w. and relative percentage (from column G).

Tab. 1 shows an overview of all substances identified in the algal strains screened. A total of 88 different substances were detected, 78 of which represent methyl esters of FAs. 36 substances were identified by their retention time according to the standard, and 40 substances were identified by their mass spectra. In such cases, the compound was identified by comparison to mass spectra with highest similarity to the proposed substance in the MS-library (Nist98 or Wiley; Tab. 2). Some methyl esters of branched FAs were detected, for example 12-methyl-14:0 or 3,7,11,15-tetramethyl-16:0. Whereas for most of the FAMEs, authentic standards or MS references were available, for some other substances only "best hit" identification was possible, for instance tetradecanamide and methyl-(9Z,12Z)-15,16-epoxy-9,12-octadecadienoate. With the help of DMOX derivatives the remaining 12 substances were identified. Unidentified substances have yet to be verified with authentic standards, which are not available at this time point. 
Results

Table 1. Overview of FAMEs and other substances identified in the microalgal strains analysed. For the FAMEs marked (**) the double bond positions are so far only tentatively identified.

\begin{tabular}{|c|c|c|}
\hline \multicolumn{3}{|l|}{88 substances, 78 methyl esters of fatty acids } \\
\hline methyl esters of saturated straight-chain FA & methyl esters of branched FA & methyl esters of monoenoic FA \\
\hline $14: 0$ & 12-methyl-14:0 & $14: 1(7 Z)$ \\
\hline $16: 0$ & 13-methyl-14:0 & $14: 1(9 Z)$ \\
\hline $17: 0$ & 14-methyl-15:0 & $15: 1(10 Z)$ \\
\hline $18: 0$ & 14-methyl-16:0 & $16: 1(3 E)$ \\
\hline 19:0 & 15-methyl-16:0 & $16: 1(5 Z)$ \\
\hline 20:0 & 3,7,11,15-tetramethyl-hexadecanoate & $16: 1(6 Z)$ \\
\hline $21: 0$ & 16 or 15 -methyl- $17: 0$ & $16: 1(7 Z)$ \\
\hline $22: 0$ & 17-methyl-18:0 & $16: 1(8 Z)$ \\
\hline 23:0 & 6,10,14-trimethyl-2-pentadecanone & $16: 1(9 Z)$ \\
\hline \multirow[t]{3}{*}{$24: 0$} & & $16: 1(11 Z)$ \\
\hline & & $17: 1(9 Z)$ \\
\hline & & $17: 1(10 Z)$ \\
\hline \multirow{2}{*}{ methyl esters of dienoic FA } & methyl esters of trienoic FA & $18: 1(9 E)$ \\
\hline & & $18: 1(9 Z)$ \\
\hline $15: 2(\mathrm{a} Z, \mathrm{~b} Z) * *$ & $16: 3(4 Z, 7 Z, 10 Z)$ & $18: 1(11 Z)$ \\
\hline $16: 2(7 Z, 10 Z)$ & $16: 3(6 Z, 9 Z, 12 Z)$ & $19: 1(11 Z)$ \\
\hline $16: 2(9 Z, 12 Z)$ & $16: 3(7 Z, 10 Z, 13 Z)$ & $20: 1(11 Z)$ \\
\hline $17: 2(7 Z, 10 Z)$ & $17: 3(\mathrm{a} Z, \mathrm{~b} Z, \mathrm{c} Z) * *$ & $22: 1(\mathrm{aZ}) * *$ \\
\hline $17: 2(9 Z, 12 Z)$ & $18: 3(5 Z, 9 Z, 12 Z)$ & $24: 1(15 Z)$ \\
\hline $18: 2(6 Z, 9 Z)$ & $18: 3(6 Z, 9 Z, 12 Z)$ & \\
\hline $18: 2(8 Z, \mathrm{x} Z)$ & $18: 3(8 Z, 11 Z, 14 Z)$ & \\
\hline $18: 2(9 E, 12 E)$ & $18: 3(9 Z, 12 Z, 15 Z)$ & \\
\hline \multicolumn{3}{|l|}{$18: 2(9 Z, 12 Z)$} \\
\hline $18: 2(11 \mathrm{Z}, 14 \mathrm{Z})$ & $19: 3(\mathrm{a} Z, \mathrm{~b} Z, \mathrm{c} Z) * *$ & \\
\hline $18: 2(9 Z, 14 Z)$ & $19: 3(\mathrm{a} Z, \mathrm{~b} Z, \mathrm{c} Z) * *$ & \\
\hline $19: 2(9 Z, 12 Z)$ & $20: 3(7 Z, 10 Z, 13 Z)$ & \\
\hline $20: 2(11 Z, 14 Z)$ & $20: 3(8 Z, 11 Z, 14 Z)$ & \\
\hline $22: 2(13 Z, 16 Z)$ & $20: 3(11 Z, 14 Z, 17 Z)$ & \\
\hline \multicolumn{3}{|l|}{ methyl esters o tetra-, penta- and hexaenoic FA } \\
\hline $16: 4(4 Z, 7 Z, 11 Z, 13 Z)$ & \multicolumn{2}{|l|}{ (8Z,11Z)-heptadeca-8,11-dienal } \\
\hline $16: 4(6 Z, 9 Z, 12 Z, 15 Z)$ & \multicolumn{2}{|c|}{ 3-(3,5-ditertbutyl-4-hydroxyphenyl) propionate } \\
\hline $18: 4(5 Z, 9 Z, 12 Z, 15 Z)$ & \multicolumn{2}{|c|}{$3,7,11,15$-tertamethyl-2-hexadecen-1-ol } \\
\hline $18: 4(6 Z, 9 Z, 12 Z, 15 Z)$ & \multicolumn{2}{|l|}{ 8-(2-octylcyclopropyl)octadecanoate } \\
\hline $19: 4(\mathrm{a} Z, \mathrm{~b} Z, \mathrm{c} Z, \mathrm{~d} Z) * *$ & \multicolumn{2}{|l|}{$2,3,4,5$-tetramethyl-3-hexen } \\
\hline $20: 4(5 Z, 8 Z, 11 Z, 14 Z)$ & \multicolumn{2}{|c|}{$(5 Z, 8 Z, 11 Z) 15,16$ epoxy $5,8,11$-octadecadienoate } \\
\hline $20: 4(8 Z, 11 Z, 14 Z, 17 Z)$ & \multicolumn{2}{|c|}{ Tetradecanamid } \\
\hline $22: 4(7 Z, 10 Z, 13 Z, 16 Z)$ & \multicolumn{2}{|l|}{ Hexadecanamide } \\
\hline $18: 5(3 Z, 6 Z, 9 Z, 12 Z, 15 Z)$ & \multicolumn{2}{|l|}{ (9Z)-Octadecenamid } \\
\hline $20: 5(5 Z, 8 Z, 11 Z, 14 Z, 17 Z)$ & \multicolumn{2}{|l|}{ 9,10-methylene tetradecanoate } \\
\hline \multicolumn{3}{|l|}{$22: 5(4 Z, 7 Z, 10 Z, 13 Z, 16 Z)$} \\
\hline \multicolumn{3}{|l|}{$22: 5(7 Z, 10 Z, 13 Z, 16 Z, 19 Z)$} \\
\hline $22: 6(4 Z, 7 Z, 10 Z, 13 Z, 16 Z, 19 Z)$ & & \\
\hline
\end{tabular}


Results

Table 2. Substances identified with GC/MS analysis. Identification of the compound was inferred from highest similarity (identification value) to the proposed substance within the MS-library. As references the MS-Nist98 library and the Wiley-library were used.

\begin{tabular}{lccc}
\hline & Nist98 & Wiley & identification value \\
\hline (8Z,11Z)-heptadeca-8,11-dienal & $\mathrm{x}$ & & 25.2 \\
3-(3,5-ditertbutyl-4-hydroxyphenyl) propionate & & $\mathrm{x}$ & 95 \\
3,7,11,15-tertamethyl-2-hexadecen-1-ol (Phytol) & $\mathrm{x}$ & & 38 \\
8-(2-octylcyclopropyl)octadecanoate & $\mathrm{x}$ & & 18.5 \\
2,3,4,5-tetramethyl-3-hexen & $\mathrm{x}$ & & 49 \\
(5Z,8Z,11Z) 15,16 epoxy 5,8,11-octadecadienoate & & $\mathrm{x}$ & 90 \\
Tetradecanamid & & $\mathrm{x}$ & 86 \\
Hexadecanamide & $\mathrm{x}$ & 91 \\
(9Z)-Octadecenamid & $\mathrm{x}$ & 99 \\
9,10 -methylene tetradecanoate & & $\mathrm{x}$ & 94 \\
\hline
\end{tabular}




\subsection{Occurrence of PUFAs within different taxonomic groups}

The comparison of profiles of lipid bound FAs from different strains of some species showed substantial similarities. In contrast profiles of different taxonomic algal genera like Synechococcus, Bangia, Cystodinium and Chlamydomonas were obviously different. In Fig. 9 FAME profiles of four species representing Cyanophyceae, Rhodophyceae, Chlorophyceae as well as Dinophyceae, respectively, is shown. The profiles demonstrate the diversity of chain-length and degree of desaturation of FAMEs among different algal taxa.
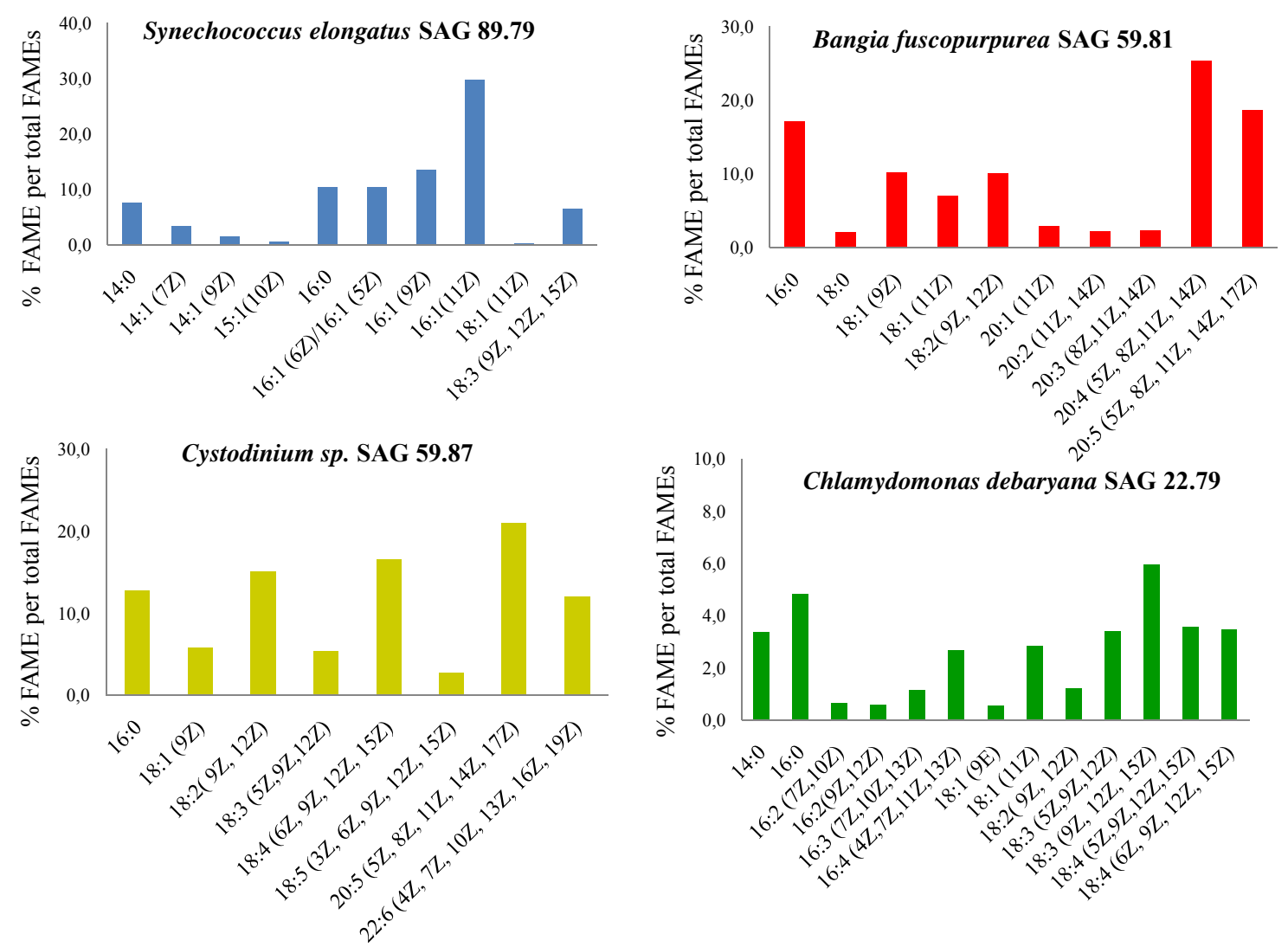

Figure 9. FAME profiles of different algal genera. Cyanophyceae are represented by Synechococcus elongatus Rhodophyceae are represented by Bangia fuscopurpurea, Dinophyceae are represented by Cystodinium sp. and Chlorophyceae are represented by Chlamydomonas debaryana. The amount of FAMEs is given in relative percentage of the total FAs. Data were obtained from one measurement. 
In addition to the FAME database a second database was established, in which the algae producing PUFAs of nutritional and economic interest were listed. This database comprises a list of PUFAs that may also be used as chemotaxonomical markers.

Strains producing a certain PUFA were collected in one table and assigned to their taxonomical group. Diagrams were developed, which illustrate the distribution of taxonomic groups producing the appropriate PUFA.

In the following paragraph, the distribution of some interesting PUFAs is described.

\section{Docosahexaenoic acid (DHA, 22:6 $\left.{ }^{(4 Z, 7 Z, 10 Z, 13 Z, 16 Z, 19 Z)}\right)$}

DHA was detected in 176 algal strains assigned to 13 different taxonomic groups. The majority of algae containing DHA belonged to the Chlorophyceae and Euglenophyceae, representing $39 \%$ and $33 \%$, respectively (Fig. 10). However, most of these algae contained only low proportions of DHA (0.1\% to $10 \%$ of total FAs). Smaller groups of algae representing Prymnesiophyceae (6\% of $\Sigma$ algae producing DHA), Xanthophyceae (2\% of $\Sigma$ algae producing DHA) and Cryptophyceae (3\% of $\Sigma$ algae producing DHA) produced DHA in even lower proportions. Tab. 3 shows the ten algal strains, for which the highest proportions of DHA were detected. Algae with high DHA proportions belonged to the group of Dinophyceae, but were also found among Euglenophyceae and Chlorophyceae. The dinophyte Ceratium horridum (generously provided by Prof. Hardeland) contained $29.3 \%$ DHA of total FAs ( $8.8 \mu \mathrm{g} / \mathrm{mg}$ d.w.), representing the highest DHA proportion in all tested microalgal strains.

Table 3. Ten algae containing highest proportions of DHA. The amount of DHA is given in percent of total FAs, data were obtained from one measurement.

\begin{tabular}{|c|c|c|c|}
\hline SAG & species & & $\%$ DHA \\
\hline & Lingulodinium & polyedrum & 14.9 \\
\hline $36-80$ & Amphidinium & klebsii & 15.4 \\
\hline $1204-18$ & Astasia & longa & 15.4 \\
\hline 5.85 & Chlorococcum & novae-angliae & 16.1 \\
\hline $37-80$ & Amphidinium & carterae & 17.3 \\
\hline 5.85 & Chlorococcum & novae-angliae & 18.8 \\
\hline 35.80 & Amphidinium & höfleri & 19.0 \\
\hline \multirow[t]{4}{*}{40.80} & Prorocentrum & cassubicum & 20.6 \\
\hline & $M E-135$ & & 22.8 \\
\hline & $M E-139$ & & 26.1 \\
\hline & Ceratium & horridum & 29.3 \\
\hline
\end{tabular}


Eicosapentaenoic acid (EPA, 20:5 $\left.5^{(5 Z, 8 Z, 11 Z, 14 Z, 17 Z)}\right)$

EPA was found in 440 algal strains belonging to 21 different taxonomic groups, predominantly Chlorophyceae, Rhodophyceae, Xanthophyceae and Euglenophyceae, representing $24 \%, 15 \%, 15 \%$ and $24 \%$ of $\Sigma$ algae producing EPA, respectively (Fig. 10). Most of the strains producing low amounts (0.3-10\%of total FAs) of EPA were representatives of the green algae (Chlorophyceae, Trebouxiophyceae, Ulvophyceae and Streptophyceae). The highest proportion of EPA was detected in algal species in the taxonomic group of Rhodophyceae, Xanthophyceae and Euglenophyceae as shown in Tab. 4. The red alga Balbania investiens contained the highest proportion of EPA $(54.2 \%$ of total FAs).

Table 4. Ten algae containing highest proportions of EPA. The amount of EPA is given in percent of total FAs, data were obtained from one measurement.

\begin{tabular}{c|ll|c}
\hline SAG & \multicolumn{1}{|c|}{ Species } & $\mathbf{\% ~ 2 0 : 5} \mathbf{( 5 Z , 8 Z , 1 1 Z , 1 4 Z , 1 7 Z )}$ \\
\hline 37.94 & Compsopogon & hookeri & 33.7 \\
29.96 & Goniochloris & sculpta & 34.6 \\
30.97 & Rhodella & violacea & 35.2 \\
39.94 & Dixoniella & grisea & 37.1 \\
101.79 & Caloglossa & leprieurii & 38.4 \\
15.97 & Characiopsis & saccata & 42,7 \\
206.80 & Audouinella & hermani & 44.5 \\
1.81 & Acrochaetium & virgatulum & 44.8 \\
106.79 & Compsopogonopsis & leptacladus & 52.4 \\
15.96 & Balbiania & investiens & 54.2 \\
\hline
\end{tabular}




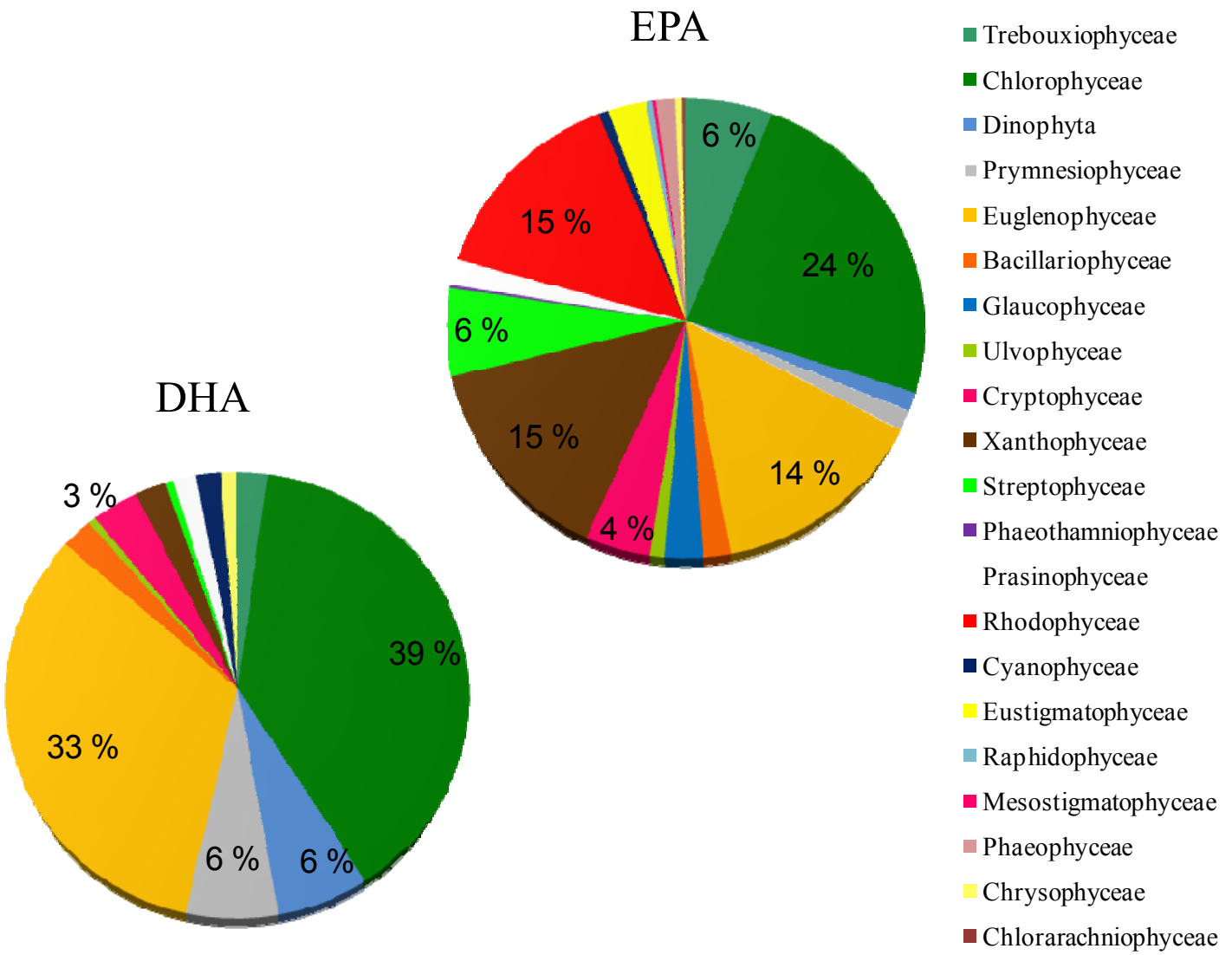

Figure 10. Overview of DHA and EPA distribution in different taxonomic groups of microalgae. The different taxonomic groups are illustrated by different colours (see legend). The proportions represent the number of species of different taxonomic groups at the SAG.

\section{Arachidonic acid (AA, 20:4 $\left.{ }^{(5 Z, 8 Z, 11 Z, 14 Z)}\right)$}

AA was found in 371 algal strains, predominantly representing Chlorophyceae, Euglenophyceae and Rhodophyceae, representing 30\%, $18 \%$ and $15 \%$ respectively (Fig. 11). AA was also found in other taxonomic groups, including Xanthophyceae $(14 \%$ of $\Sigma$ algae producing AA), Trebouxiophyceae ( $8 \%$ of $\Sigma$ algae producing AA), Streptophyceae (5\% of $\Sigma$ algae producing AA) and Phaeophyceae (2\% of $\Sigma$ algae producing AA). The AA proportions of most strains representing the Chlorophyceae was very low (0.2-10\% of total FAs). Algae with higher content of AA ( $>13 \%$ of total FAs) belonged to the group of Rhodophyceae, Euglenophyceae, Xanthophyceae and Trebouxiophyceae. In Tab. 5 the ten algal strains with the highest proportions of AA are shown. Eight strains belong to the Rhodophyceae with Pseudochantransia sp. harbouring $67 \%$ of AA. The other two strains belong to green algae (Trebouxiophyceae and 
Chlorophyceae). The chlorophycean alga Palmodictyon varium contained the highest amount of AA with $73.8 \%$ of total FAs (102 $\mu \mathrm{g} / \mathrm{mg}$ d.w., Tab. 5).

Table 5. Ten algae containing highest proportions of AA. The amount of AA is given in percent of total FAs, data were obtained from one measurement.

\begin{tabular}{c|ll|c}
\hline SAG & \multicolumn{1}{|c|}{ Species } & $\mathbf{\% ~ 2 0 : 4 ~ ( 5 Z , 8 Z , 1 1 Z , 1 4 Z ) ~}$ \\
\hline 19.95 & Trochisciopsis & tetraspora & 39,9 \\
14.96 & Pseudochantransia & sp & 42.1 \\
111.79 & Porphyridium & aerugineum & 42.2 \\
$1380-1 \mathrm{e}$ & Porphyridium & purpureum & 42.7 \\
52.94 & Audouinella & eugenea & 48.3 \\
$19-96$ & Pseudochantransia & sp & 51.4 \\
2043 & Myrmecia & bisecta & 51.7 \\
46.94 & Thorea & ramosissima & 52.4 \\
19.96 & Pseudochantransia & sp & 67.1 \\
3.92 & Palmodictyon & varium & 73.8 \\
\hline
\end{tabular}

\section{Octadecapentaenoic acid $\left(18: 5^{(3 Z, 6 Z, 9 Z, 12 Z, 15 Z)}\right)$}

The FA 18:5n-3 was found in 12 strains representing four different taxonomic groups: Dinophyceae (42\% of $\Sigma$ algae producing 18:5n-3), Prymnesiophyceae (42\% of $\Sigma$ algae producing 18:5n-3), Chrysophyceae (8\% of $\Sigma$ algae producing 18:5n-3) and Rhodophyceae (8\% of $\Sigma$ algae producing 18:5n-3, Fig. 11). In Tab. 6 the ten algae with the highest proportions of 18:5n-3 are listed. The highest amount of 18:5n-3 was detected for the dinophyte Lingulodinium polyedrum (generously provided by Prof Hardeland) with $22.7 \%$ of total FAs (18 $\mu \mathrm{g} / \mathrm{mg}$ d.w.).

Table 6. Ten algae containing highest proportions of 18:5n-3. The amount of 18:5n-3 is given in percent of total FAs, data were obtained from one measurement.

\begin{tabular}{c|ll|c}
\hline SAG & species & \% 18:5 (3Z,6Z,9Z,12Z,15Z) \\
\hline $912-1$ & Coccolithophora & $s p$ & 1,7 \\
59.87 & Cystodinium & $s p$ & 2,8 \\
& Ceratium & horridum & 3,2 \\
$927-3$ & Isochrysis & sp & 4,0 \\
18.97 & Prymnesium & saltans & 4,6 \\
13.92 & Isochrysis & galbana & 5,9 \\
127.79 & Prymnesium & parvum & 10,5 \\
& ME-139 & & 20,0 \\
& ME-135 & & 21,2 \\
& Lingulodinium & polyedrum & 22,7 \\
\hline
\end{tabular}


$\gamma$-Linolenic acid (GLA, 18:3 $\left.{ }^{(6 Z, 9 Z, 12 Z)}\right)$

408 algal strains representing 16 different taxonomic groups contain GLA. The majority of algal strains containing high levels of GLA belong to the Chlorophyceae, representing $64 \%$ of $\Sigma$ algae producing GLA (Fig. 11). In addition to Chlorophyceae GLA was also present in FA profiles of Cyanophyceae and Streptophyceae, representing $10 \%$ and $13 \%$ respectively (Fig. 11). The highest proportion of GLA was found in the ten strains which are shown in Tab. 7. These algae represent Cyanophyceae, Chlorophyceae and Trebouxiophyceae. The trebouxiophycean alga Coenocystis inconstans showed the highest amount of GLA with $35.3 \%$ of total FAs $(45.7 \mu \mathrm{g} / \mathrm{mg}$ d.w.).

Table 7. Ten algae containing highest proportions of GLA. The amount of GLA is given in percent of total FAs, data were obtained from one measurement.

\begin{tabular}{c|ll|c}
\hline SAG & \multicolumn{1}{|c|}{ Species } & \% 18:3 (6Z,9Z,12Z) \\
\hline $257-80$ & Spirulina & platensis & 19,7 \\
3.87 & Cylindrocapsa & geminella & 21.3 \\
46.80 & Microcystis & aeruginosa & 22.0 \\
$211-32$ & Schroederia & sorokiniana & 22.0 \\
25.95 & Deasonia & multinucleata & 22.1 \\
49.88 & Spirulina & maxima & 24.6 \\
26.91 & Desmodesmus & multiformis & 26.9 \\
Müller $9+$ & Chlorogonium & sp & 27.8 \\
Hg 1973-375 & Chlorococcum & sp & 28.5 \\
2040 & Coenocystis & inconstans & 35.3 \\
\hline
\end{tabular}

\section{Pinolenic acid $\left(18: 3^{(5 Z, 9 Z, 12 Z)}\right)$}

Pinolenic acid was found in 32 strains representing four different taxonomic groups: Chlorophyceae (91\% of $\Sigma$ algae producing pinolenic acid), Prymnesiophyceae (3\% of $\Sigma$ algae producing pinolenic acid), Dinophyceae (3\% of $\Sigma$ algae producing pinolenic acid) and Cryptophyceae (3\% of $\Sigma$ algae producing pinolenic acid) (Fig. 11). The majority of the strains were assigned to the genus Chlamydomonas. Thus the strains with the highest proportion of 18:3 ${ }^{(5 Z, 9 Z, 12 Z)}$ were Chlamydomonas strains (Tab. 8) among two other species. The chlorophycean alga Chlamydocapsa sp. displayed the highest amount of $18: 3^{(5 Z, 9 Z, 12 Z)}$ with $12.2 \%$ of total FAs $(4.3 \mu \mathrm{g} / \mathrm{mg}$ d.w.) (Tab. 8$)$ 
Table 8. Ten algae containing highest proportions of $18: 3^{(5 Z, 9 Z, 12 Z)}$. The amount of $18: 3^{(5 Z, 9 Z, 12 Z)}$ is given in percent of total FAs, data were obtained from one measurement.

\begin{tabular}{c|ll|c}
\hline SAG & species & $\mathbf{\% 1 8 : 3}(\mathbf{5 Z , 9 Z , 1 2 Z )}$ \\
\hline 14.72 & Chlamydomonas & debaryana & 5,9 \\
126.79 & Apistonema & sp & 6,1 \\
7.79 & Chlamydomonas & debaryana & 7,3 \\
6.79 & Chlamydomonas & debaryana & 7,6 \\
4.72 & Chlamydomonas & debaryana & 8,9 \\
22.79 & Chlamydomonas & debaryana & 9,5 \\
11.73 & Chlamydomonas & debaryana & 11,3 \\
$11-55 b$ & Chlamydomonas & debaryana & 11,9 \\
58.80 & Chlamydocapsa & sp & 12,2 \\
\hline
\end{tabular}

\section{Hexadecatetraenoic acid $\left(16: 4^{(4 Z, 7 Z, 10 Z, 13 Z)}\right)$}

762 SAG algal strains contain $16: 4^{(4 Z, 7 Z, 10 Z, 13 Z)}$. Most of these strains were representatives of the Chlorophyceae with $81 \%$ and Euglenophyceae with $7 \%$ of all algal strains producing 16:4n-3 (Fig. 11). Other algae were representatives of e.g. Trebouxiophyceae (4\% of $\Sigma$ algae producing 16:4n-3) and Prasinophyceae (2\% of $\Sigma$ algae producing 16:4n3). The highest proportions of 16:4n-3 were mainly found in chlorophycean algae, especially the genus Chlamydomonas. The ten algae with the highest proportion of 16:4n-3 belonged to the Chlorophyceae, and two candidates to the Euglenophyceae and Trebouxiophyceae (Tab. 9). The chlorophycean alga Polytoma sp. displayed the highest amount with $52.5 \%$ of total FAs $(2.8 \mu \mathrm{g} / \mathrm{mg}$ d.w. $)$

Table 9 Ten algae containing highest proportions of 16:4n-3. The amount of $16: 4 n-3$ is given in percent of total FAs, data were obtained from one measurement.

\begin{tabular}{c|ll|c}
\hline SAG & species & $\mathbf{\%} \mathbf{1 6 : 4} \mathbf{( 4 Z , 7 Z , 1 0 Z , 1 3 Z )}$ \\
\hline 78.94 & Chlamydomonas & sp & 24,1 \\
$1244-5$ & Lepocinclis & ovata & 24,7 \\
$11-49$ & Chlamydomonas & leiostracta & 26,7 \\
Sep 93 & Haematococcus & buetschlii & 27,4 \\
76.80 & Nautococcus & soluta & 28,8 \\
26.90 & Chloromonas & rosae & 30,0 \\
$11-41$ & Chlamydomonas & asymmetrica & 32,7 \\
$251-2$ & Nannochloris & sp. & 42,3 \\
25.86 & Chlamydomonas & zebra & 45,3 \\
$62-27$ & Polytoma & sp & 52,5 \\
\hline
\end{tabular}


Hexadecatetraenoic acid $\left(16: 4^{(6 Z, 9 Z, 12 Z, 15 Z)}\right)$

For only a few microalgae strains the FA 16:4n-1 was found. These four strains represent the class of Dinophyceae and Xanthophyceae. In Tab. 10 the four strains producing 16:4n1 are shown. The dinophycaen alga Ceratium horridum contained the highest proportion of $16: 4 \mathrm{n}-1$ with $6.6 \%$ of total FAs $(2 \mu \mathrm{g} / \mathrm{mg}$ d.w. $)$.

Table 10. Algae containing highest proportions of 16:4n-1. The amount of $16: 4 n-1$ is given in percent of total FAs, data were obtained from one measurement.

\begin{tabular}{c|ll|c}
\hline SAG & species & 16:4 (6Z,9Z,12Z,15Z) \\
\hline \multirow{2}{*}{28.87} & Pyrenomonas & helgolandii & 1,1 \\
& ME139 & & 1,6 \\
2002 & Pyrenomonas & salina & 3,9 \\
& Ceratium & horridum & 6,6 \\
\hline
\end{tabular}

\section{4-methylpentadecanoic acid (14-methyl-15:0)}

The methyl-branched fatty acid 14-methyl-15:0 was detected in 43 strains assigned to Euglenophyceae (53\% of $\Sigma$ algae producing 14-methyl-15:0), Chlorophyceae (44\% of $\Sigma$ algae producing 14-methyl-15:0) and Xanthophyceae (2\% of $\Sigma$ algae producing 14methyl-15:0) (Fig. 9). In most of the chlorophytes only low amounts of 14-methyl-15:0 (0.5-1.4\%) were detected. Strains with higher abundance of the methyl branched fatty acid belonged to the Euglenophyceae and some to Xanthophyceae (Tab. 11). The euglenophyte Euglena gracilis SAG 1224-5/19 had the highest amount with $11 \%(0.3 \mu \mathrm{g} / \mathrm{mg}$ d.w.) (Tab. 11). 
Results

Table 11. Ten algae containing highest proportions of 14-methyl-15:0; The amount of 14-methyl-15:0 is given in percent ratios, data were obtained from one measurement.

\begin{tabular}{c|ll|c}
\hline SAG & species & \% 14-methyl-15:0 \\
\hline 31.95 & Characiochloris & acuminata & 3,5 \\
$1224-5 / 18$ & Euglena & gracilis & 3,9 \\
$1283-10$ & Trachelomonas & lefévrei & 4,1 \\
2015 & Amphikrikos & sp & 4,2 \\
$237-1$ & Chlorococcum & infusionum & 4,3 \\
$813-1$ & Pleurochloris & meiringensis & 4,5 \\
$1224-5 / 11$ & Euglena & gracilis & 4,9 \\
$1204-26 a$ & Distigma & proteus & 5,0 \\
$1247-1$ & Menoidium & bibacillatum & 7,2 \\
$1224-5 / 19$ & Euglena & gracilis & 11,0 \\
\hline
\end{tabular}



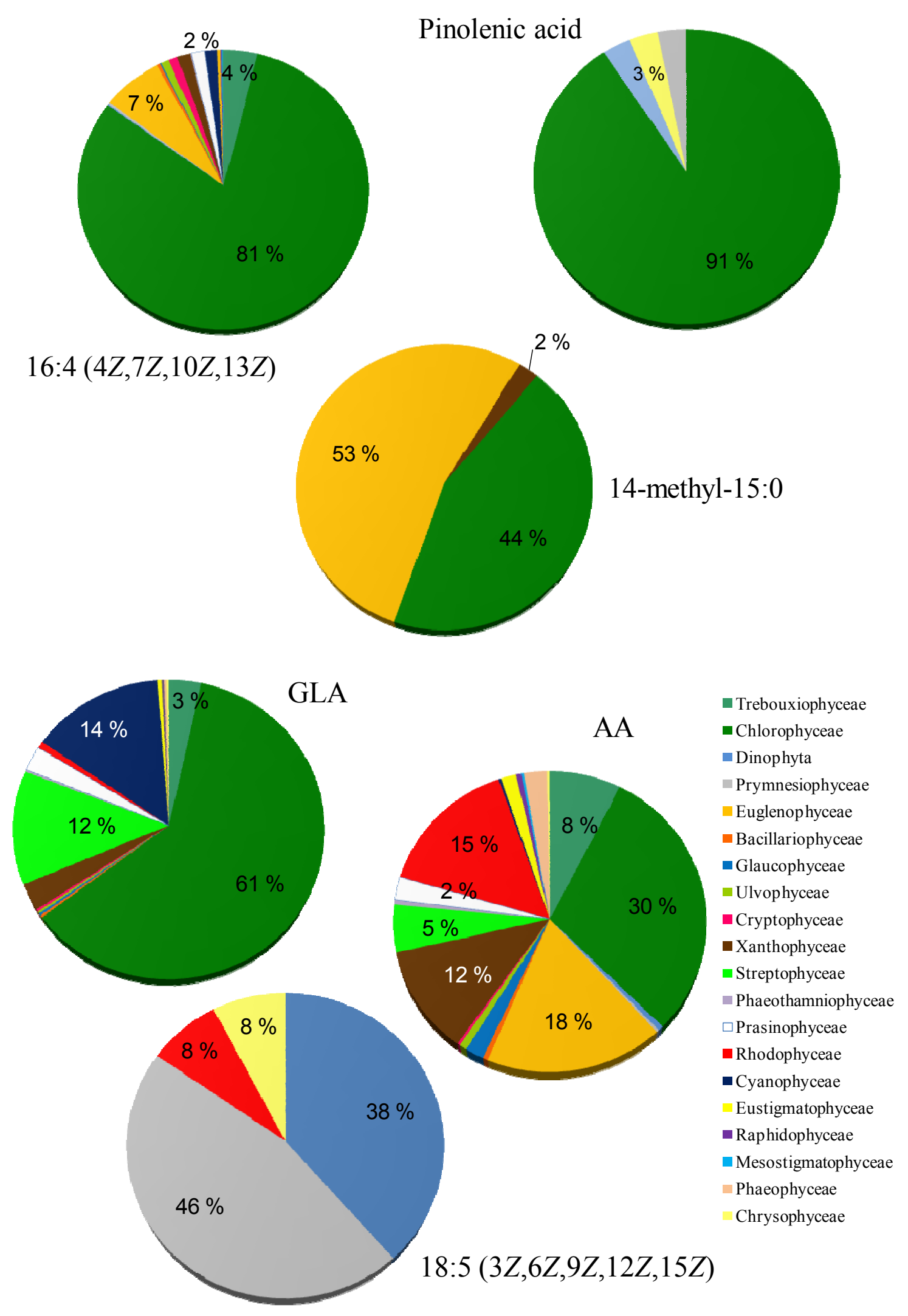

Figure 11. Overview of AA, GLA, 18:5n-3, pinolenic acid, 16:4n-3 and 14-methyl-15:0 distribution in different taxonomic groups of microalgae. The different taxonomic groups are illustrated by different colours (see legend). The proportions represent the number of species of different taxonomic groups at the SAG. 


\subsection{Oxylipin profiling of SAG microalgal strains}

In addition to the FAME screening it was another aim of this thesis to identify and quantify oxylipins in all SAG microalgal strains. For this purpose the FAME extracts of the algae, previously used for the FAME analysis were prepared as described in section II-10.12. The samples were subjected to GC/MS to generate the mass spectrum of every single substance. The mass spectra of whole GC chromatograms obtained were extracted for fragments which were specific for silylated products. The mass spectra of signals representing silylated products were characterised by the ion $\mathrm{m} / \mathrm{z} 73$, which derived from the derivatisation reagent TMS. The mass spectra of signals representing methoxylated products were characterised by ions resulting from specific fragmentation at the $\mathrm{C}$-atom carrying the methoxy-group. As references the MS-Nist98 library was used. Those compounds, that were identified by this prescreen were identified by comparison to mass spectra with highest similarity to the proposed substance in the MS-library (Nist98).

The oxylipin screening started by analysing 78 extracts of cyanobacterial strains. The results of GC/MS analysis showed that the FID signals decreased in comparison to the prior GC-analysis of FAMEs and that no additional substances were detected (data not shown). The comparison of mass spectra obtained with mass spectra of the MS-Nist98 library revealed that plasticiser, such as phthalates were predominantly identified as "best hit". These results indicated that the amount of oxylipins in the starting material was not enough and the protocol used might not be suitable for oxylipin analysis of microalgae. Moreover it remained unclear whether microalgae in stationary growth phase contain oxylipins in sufficient quantities.

Therefore the oxylipin screening as described above was not continued but another approach was followed for further investigations. For this purpose genomic sequences of different microalgae were searched resembling enzymes catalysing the synthesis of oxylipins. Results of the isolation and characterisation of PUFA metabolising enzymes is described in more detail in section III-4. 


\section{Investigation of the biosynthetic pathway for $18: 5 n-3$}

One PUFA identified as a putative chemotaxonomical marker, was 18:5n-3, which is of particular interest, because its biosynthetic pathway is not known. Two routes of 18:5n-3 biosynthesis are possible, 18:5n-3 may either derive from further desaturation of 18:4n-3 by a $\Delta 3$-desaturase or from partial $\beta$-oxidation of EPA (Fig. 1). To address the biosynthesis of 18:5n-3, suitable algal strains were chosen for the isolation of 18:5n-3 (10.11) and the identification of its absolute structure by GC/MS (10.7) and NMR (10.10) analysis. In addition, lipid analysis and labelling experiments were performed (10.14) to clarify the biochemical pathway for the generation of 18:5n-3 in vivo.

\subsection{Selection of algae strains suitable for 18:5n-3 isolation}

Based on the large screen, several algal strains were identified, which produce $18: 5 n-3$ in high proportions and which were easy to cultivate. Most of these candidate strains belong to the class of Dinophyceae and Haptophyceae (Fig. 11). Because dinophytes are sensitive to culture conditions and exhibit only modest growth rates no Dinophyceae were selected for production of 18:5n-3. Instead, two closely related haptophycean strains were chosen: Prymnesium parvum SAG 127.79 and Prymnesium saltans SAG 18.97. The FAME profiles of both strains are shown in Fig. 12. The main PUFAs in both strains were 18:4n-3 $(12 \mu \mathrm{g} / \mathrm{mg}$ d.w. in SAG 127.79 and $7.4 \mu \mathrm{g} / \mathrm{mg}$ d.w. in SAG 18.97) and 22:6n-3 (11.8 $\mu \mathrm{g} / \mathrm{mg}$ d.w. in SAG 127.79 and $10.8 \mu \mathrm{g} / \mathrm{mg}$ d.w. in SAG 18.97). The PUFA 18:5n-3 was also present in relatively high amounts in both strains with $10.5 \mu \mathrm{g} / \mathrm{mg} \mathrm{d}$.w. in SAG 127.79 and $4.6 \mu \mathrm{g} / \mathrm{mg}$ d.w. in SAG 18.97. 


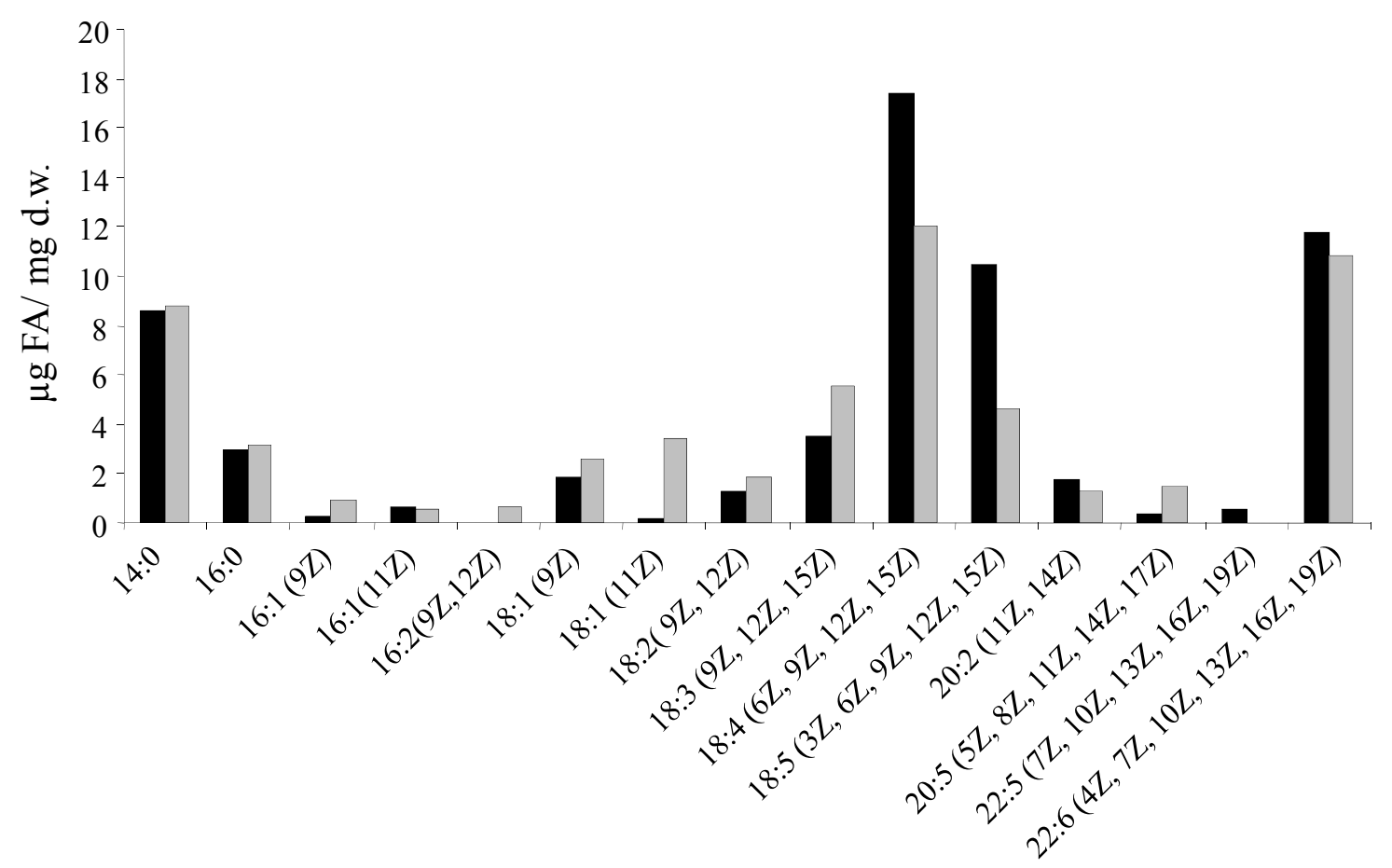

Figure 12. FAME profiles of $\boldsymbol{P}$. parvum and $\boldsymbol{P}$. saltans. Grey bars represent FAMEs of $P$. saltans and black bars represent FAMEs of $P$. parvum. FAMEs were extracted via alkaline hydrolysis and analysed and identified with GC and GC/MS. The amounts of FAMEs were calculated as $\mu \mathrm{g}$ FAME per mg dry weight. The experiment was repeated three times using acidic hydrolysis and showed similar results.

For further experiments the two selected algal strains were cultivated recording optical densities of $\mathrm{OD}_{600}=0.1-0.4$ (Fig. 13). The cultures grew relatively slowly, after 25 days they reached an optical density of 0.4 . For the preparation of 18:5n-3 the cultures grew until they reached an $\mathrm{OD}_{600}$ of 1.2. 


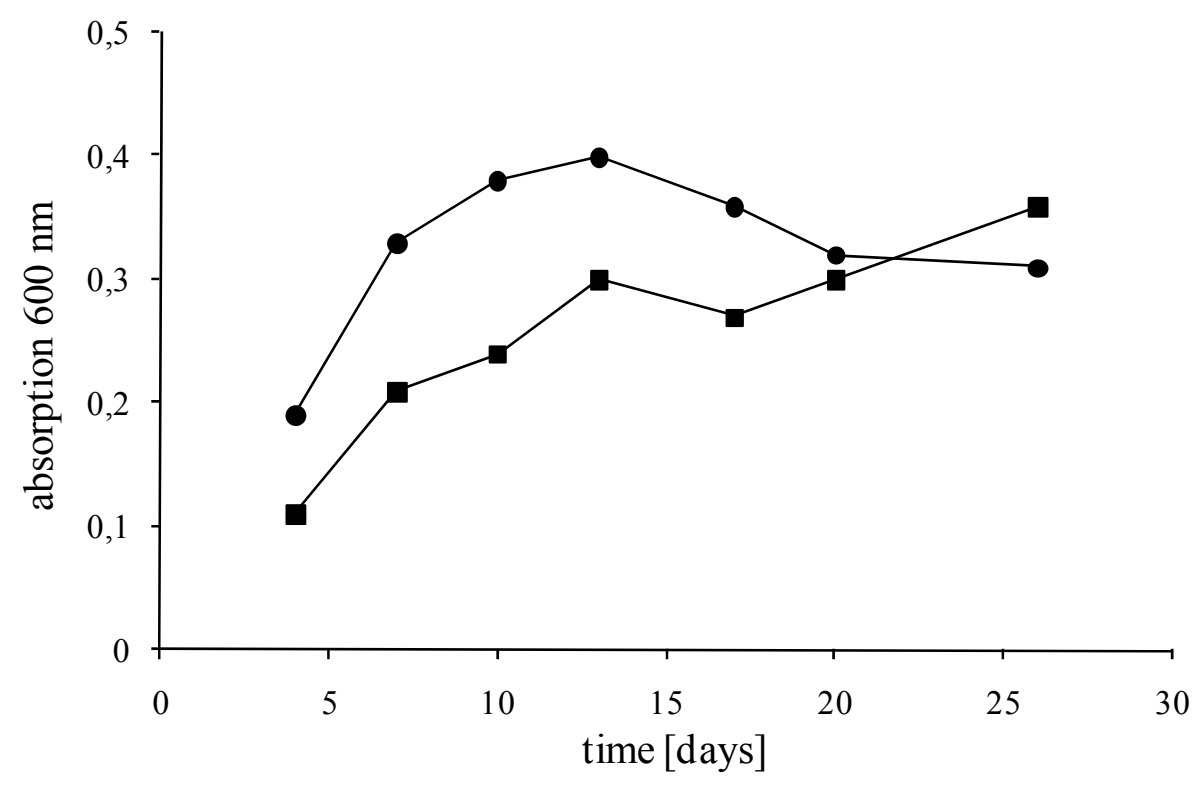

Figure 13. Growth curve of $\boldsymbol{P}$. saltans and $\boldsymbol{P}$. parvum. The growth curves are shown for one representative out of two experiments. $\mathbf{\square}=$ P. saltans, $\bullet=P$. parvum

\subsection{Lipid analysis of $P$. saltans SAG 18.97}

The analysis of 18:5n-3 biosynthesis included the analysis of FAs bound to the different lipid classes in the selected algae. Therefore the lipids of $P$. saltans were isolated and then separated by TLC (10.1). Each lipid was scraped out and the lipid bound FAs were identified via GC. Lipid analysis of $P$. parvum was done by Mareike Hoffmann (Göttingen) and showed similar results to that of $P$. saltans. Therefore only the data of $P$. saltans are described here.

Fig. 14 shows the analytical TLC plates of neutral-, glyco- and phospholipids. In general the neutral lipids represented the major fraction, followed by the glycolipid fraction. The least respected fraction consisted of phospho- and betaine lipids. The main neutral lipids of P. saltans were TAG and DAG, both indicated by an arrow. Among glycolipids MGD, DGD and SQD were detected, with MGD as the dominant lipid. An additional glycolipid on the preparative TLC plate was identified as a sphingolipid. In addition to PC and the betaine lipid DGTS the group of phospholipids contained two unknown betaine lipids, which were detected by the Dragendorff reagent. All FAs bound to the identified lipids were extracted via acidic hydrolysis (10.3) and the corresponding FAMEs were analysed by GC (10.6). 


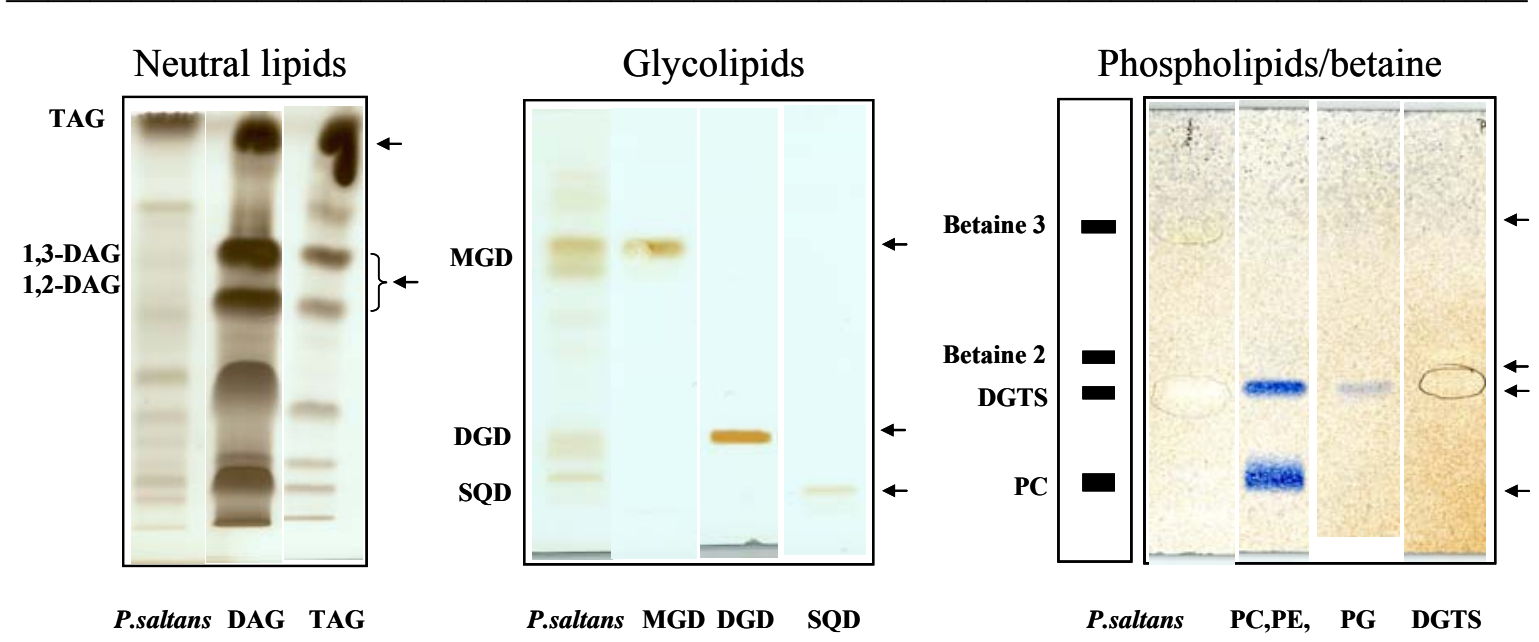

Figure 14 Analysis of lipid classes isolated from $P$. saltans by TLC. Lipids were developed as described in 10.1. Standards for neutral lipids: diacylglycerol (1,3-DAG and 1,2-DAG); triacylglycerol (TAG), standards for glycolipids: digalactosyldiacylglycerol (DGD), monogalactosyldiacylglycerol (MGD), sulfoquinovosyldiacylglycerol (SQD); standards for phospholipids: diacylglycerol- N,N,N-trimethylhomoserine (DGTS), phosphatidylcholine (PC), phosphatidylethanolamine (PE), phosphatidylglycerol (PG) and. These data represent a single experiment.

The FA composition of the lipids purified is given in Tab. 12. The main FAs in TAG were 14:0 (25\% of total FA), 16:0 (14\% of total FA), 18:1 (9Z) (22\% of total FA) and 18:4n-3 (10\% of total FA). Other C18 FAs bound to TAG were ALA (9\% of total FA), LA (7 \% of total FA) and 18:5n-3 (6\% of total FA). Minor components were 16:1 (9Z), 16:2, 18:0, 20:3n-3 and EPA. The main FAs in DAG were 14:0 (25 \% of total FA), 16:0 (14 \% of total FA) and 18:1 (9Z) (20\% of total FA). In addition, 18:0 (13\% of total FA) and 20:3n-3 ( $9 \%$ of total FA) were identified. Among glycolipids the FAME compositions of MGD and DGD were similar. For both lipids the most abundant FAs were 14:0 (24 \% and $21 \%$ of total FA), $18: 4 n-3$ (16\% and $24 \%$ of total FA) and $18: 5 n-3(22 \%$ and $19 \%$ of total FA). In addition 16:1 (7Z) and 18:1 (9Z) were found in relatively high proportions in MGD. FAs represented to a minor degree were 16:1 (9Z), 16:2, 18:0, LA and ALA. In DGD 18:1 (11Z) (16\% of total FA) were additionally recovered in higher amounts. Other minor components were 16:1 (7Z) (6\% of total FA), 16:2 (1\% of total FA), LA (4\% of total FA) and ALA (9\% of total FA). C20 FAs were identified neither bound to MGD nor to DGD. The main FAs bound to SQD were 14:0 (36\%), 16:1 (7Z) (22\%) and ALA (26\%). Minor amounts of 18:1 (9Z), LA, 18:4n-3 and DHA could be found. No 18:5n-5 was bound to SQD. The FAs esterified to sphingolipids were 14:0 (46\%), 16:1 (7Z) 
Results

(21\%), 16:1 (9Z) (23\%) and 18:0. Unsaturated C18 FAs and C20 FAs were not found. The dominant FAs bound to PC were $16: 0$ with $29 \%$ and DHA with $21 \%$. Other major FAs were 14:0 (11\%) and 18:5n-3 (16\%). For PE the major FA was 18:1 (11Z) making up $61 \%$ of all FAs bound to PE. Further FAMEs identified were 14:0, 16:0, 16:1 (9Z), 16:2, 18:0 and 18:1 (9Z). The distribution of FAs within the betaine lipids was very similar to that of PC and PE. The most abundant FAMEs were DHA and 16:0. 18:5n-3 and EPA were detected in DGTS, but not in the other betaine lipids.

Overall the results indicate that the unusual FA 18:5n-3 was found in all three main lipid classes, however the major portion was esterified to glycolipids. 
Table 12. FAME composition of neutral-, glycol-, phospho- and betaine lipids purified of $\boldsymbol{P}$. saltans. The data were obtained from one measurement. The relative amounts of FAME are given in percent of total FAs.

\begin{tabular}{|c|c|c|c|c|c|c|c|c|c|c|c|c|c|c|c|c|}
\hline \multicolumn{2}{|c|}{$\begin{array}{l}\text { Lipids } \\
\text { (\% of total FAs) }\end{array}$} & \multirow{2}{*}{$\begin{array}{l}14: 0 \\
25\end{array}$} & \multirow{2}{*}{$\begin{array}{c}16: 0 \\
14\end{array}$} & \multirow[t]{2}{*}{$16: 1(7 Z)$} & \multirow{2}{*}{$\begin{array}{l}16: 1(9 Z) \\
2\end{array}$} & \multirow{2}{*}{$\begin{array}{l}\begin{array}{l}16: 2 \\
(7 Z, 10 Z)\end{array} \\
1\end{array}$} & \multirow{2}{*}{$\begin{array}{l}\text { 18:0 } \\
1\end{array}$} & \multirow{2}{*}{$\begin{array}{l}\mathbf{1 8 : 1} \\
(\mathbf{9 Z )}\end{array}$} & \multirow[t]{2}{*}{$\begin{array}{l}18: 1 \\
(11 Z)\end{array}$} & \multirow{2}{*}{$\begin{array}{l}18: 2 n-6 \\
7\end{array}$} & \multirow{2}{*}{$\begin{array}{l}18: 3 n-3 \\
9\end{array}$} & \multirow{2}{*}{$\begin{array}{l}18: 4 n-3 \\
10\end{array}$} & \multirow{2}{*}{$\begin{array}{l}18: 5 n-3 \\
6\end{array}$} & \multirow{2}{*}{$\begin{array}{l}20: 3 n-6 \\
1\end{array}$} & \multirow{2}{*}{$\begin{array}{l}20: 5 n-3 \\
1\end{array}$} & \multirow[t]{2}{*}{$22: 6 n-3$} \\
\hline NL & TAG & & & & & & & & & & & & & & & \\
\hline & DAG & 25 & 33 & & & & 13 & 20 & & & & & & 9 & & \\
\hline \multirow[t]{4}{*}{ GL } & MGD & 24 & & 10 & 1 & 3 & 1 & 12 & & 5 & 5 & 16 & 22 & & & \\
\hline & DGD & 21 & & 6 & & 1 & & & 16 & 4 & 9 & 24 & 19 & & & \\
\hline & SQD & 36 & & 22 & & & & 3 & & 3 & 26 & 6 & & & & 4 \\
\hline & $\begin{array}{l}\text { Sphingo- } \\
\text { sin }\end{array}$ & 46 & & 21 & 23 & & 10 & & & & & & & & & \\
\hline \multirow[t]{2}{*}{ PL } & PC & 11 & 29 & & & & 3 & 2 & 8 & & 2 & 7 & 16 & & 1 & 21 \\
\hline & PE & 3 & 10 & & 3 & 5 & 7 & 5 & 61 & & & & & & 7 & \\
\hline \multirow[t]{3}{*}{ BL } & Betaine 2 & 1 & 12 & & 1 & & 3 & 4 & 1 & & 1 & 1 & & & & 76 \\
\hline & DGTS & 5 & 19 & 1 & 1 & & 3 & 3 & 7 & & 2 & 4 & 10 & & 1 & 45 \\
\hline & Betaine 3 & & 31 & & & & 7 & 3 & 5 & & & & & & & 54 \\
\hline
\end{tabular}




\subsection{Preparation of 18:5n-3 for further analysis}

For the purification of 18:5n-3 the two Prymnesium strains were cultivated as $300 \mathrm{ml}$ cultures and harvested in the late exponential growth phase. The FAMEs extracted were separated via RP-HPLC (10.11), and an aliquot of the me18:5n-3 containing fraction was analysed via GC/MS (Fig. 15). A comparison of mass spectra obtained with mass spectra from publically available reference data at "The lipid library" of The Scottish Crop Science research Institute (http://www.lipidlibrary.co.uk/masspec.html) showed that the isolated FAME had 18 C-atoms with 5 double bonds. The appropriate mass of me18:5n-3 is $m / z$ 288. The preparation of DMOX derivatives of 18:5n-3 failed, no peak representing 18:5n-3 was present in the GC/MS chromatogram. In addition, the preparation of picolinyl ester (10.5) of 18:5n-3 was not successful, too. The results indicated that the formation of the DMOX and picolinyl headgroup was not possible due to the $\Delta 3$ double bond close to the carboxy end. Furthermore the preparation of DMOX and picolinyl derivatives might lead to an unstable product, which was not detectable.

The absolute configuration of 18:5n-3 was therefore determined by NMR-analysis (10.10). The amount of purified me18:5n-3 was determined by co injection of a defined amount of methylated LA (meLA) as a standard. The preparation yielded a total of $50 \mu \mathrm{g}$ of me18:5n-3, which were subjected to NMR analysis (2.4). 


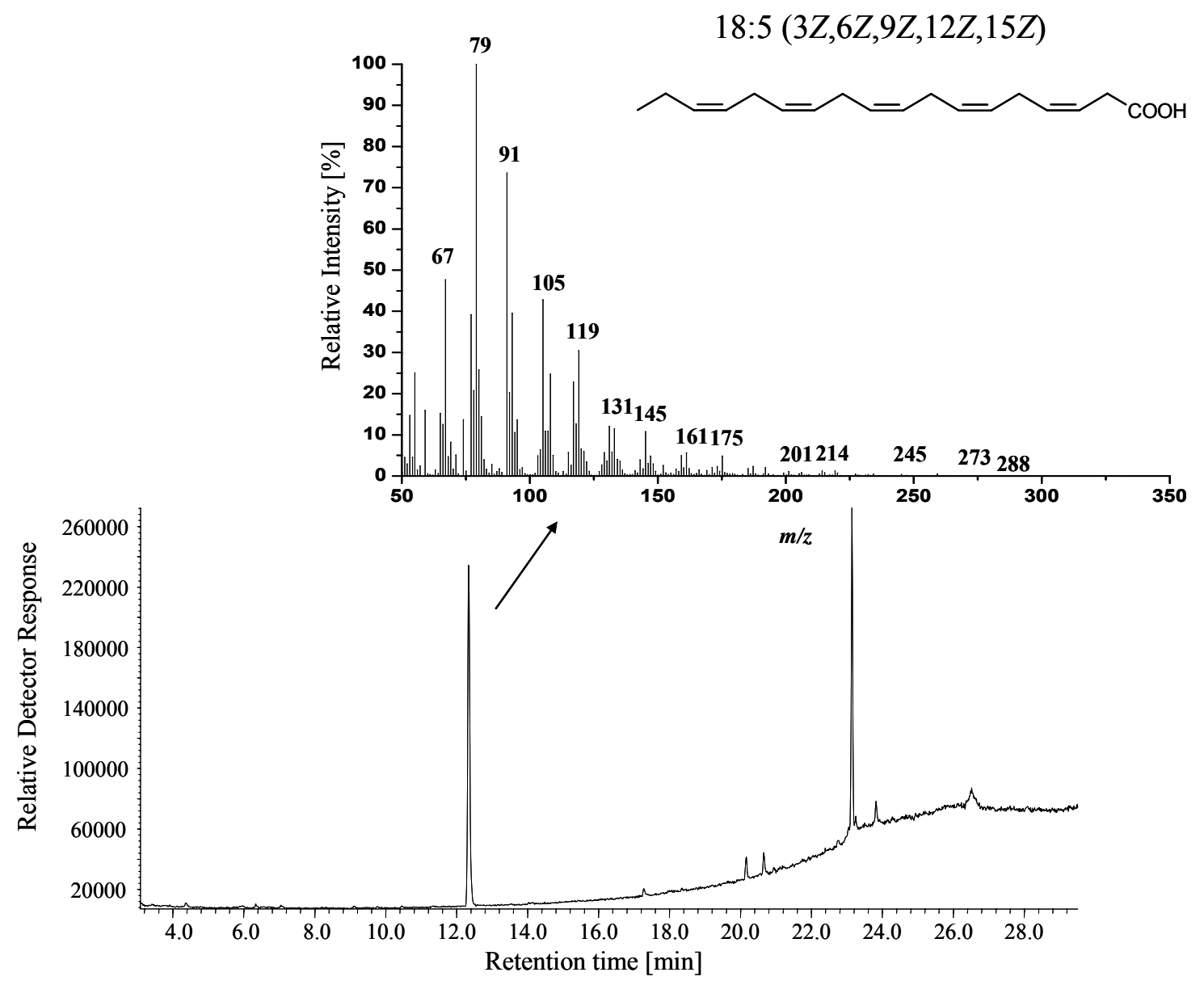

Figure 15. GC/MS-Analysis of 18:5n-3 isolated via RP-HPLC. The GC-elution profile and mass spectrum of the main peak is shown. The mass spectrum represents a FA with $18 \mathrm{C}$-atoms and 5 double bonds $\rightarrow 288 \mathrm{~m} / \mathrm{z}$.

\subsection{Analysis of 18:5n-3 structure by NMR}

It was already demonstrated with GC/MS analysis, that $P$. parvum and $P$. saltans harbour a FA with $18 \mathrm{C}$-atoms and 5 double bonds. However, it was not possible to determine the positions of the double bonds by analysis of DMOX derivatives. Also, the stereo configuration of the double bonds remained unclear. Therefore, the isolated me18:5 was used for NMR analysis. The analysis was performed by Dr. Andrea Porzel (LeibnizInstitute of Plant Biochemistry in Halle/Saale).

The relevant aspects of the NMR data obtained are shown in Tab. 13. The coupling constant of $\mathrm{J}(\mathrm{H}-3 / \mathrm{H}-4)$ of $10.6 \mathrm{~Hz}$ indicates a $\Delta 3$ double bond in cis configuration. The ${ }^{13} \mathrm{C}$-data also demonstrate, that the closest double bond to the carboxy end is located between $\mathrm{C}-3$ and $\mathrm{C}-4\left(\delta^{13} \mathrm{C}[\mathrm{ppm}]=121.2-131.3\right)$ and the closest double bond to the methyl end between $\mathrm{C}-15$ and $\mathrm{C}-16\left(\delta^{13} \mathrm{C}[\mathrm{ppm}]=127.0-132.0\right)$. 
Table 13. NMR data of 18:5n-3

\begin{tabular}{|c|c|c|c|c|}
\hline Pos & $\begin{array}{l}\delta{ }^{1} \mathrm{H} \text { (Armbrust et } \\
\text { al. })^{\mathrm{a}} \mathrm{M} \text { (J(Kajikawa } \\
\text { et al. }))\end{array}$ & $\begin{array}{l}\delta^{13} \mathrm{C} \\
{[\mathrm{ppm}]^{\mathrm{b}}}\end{array}$ & $\mathrm{a}$ & $\begin{array}{l}{ }^{1} \mathrm{H} \text {-chemical shifts without multiplicity and } \\
\text { derive from 2D-NMR-spectra }\end{array}$ \\
\hline 1 & --- & 172.2 & $\mathrm{~b}$ & ${ }^{13} \mathrm{C}$-chemical shifts derive from hetero \\
\hline 2 & $3.133 \mathrm{~d}(5.8)$ & 32.7 & & nucleic 2D-NMR-Spectra \\
\hline 3 & 5.59 & 121.2 & $\mathrm{c}$ & $-\mathrm{CH}=: \delta^{1} \mathrm{H} \quad 5.44-5.28 \mathrm{ppm}$ \\
\hline 4 & 5.58 & 131.3 & d & $-\mathrm{CH}_{2}-: \delta{ }^{1} \mathrm{H} \quad 2.87-2.80 \mathrm{ppm}$ \\
\hline 5 & 2.83 & 25.6 & e & $-\mathrm{CH}=: \delta{ }^{13} \mathrm{C} \quad 128.9-127.1 \mathrm{ppm}$ (derive \\
\hline 6 & $\begin{array}{l}c \\
c\end{array}$ & $\mathrm{e}$ & & from heteronucleic 2D-NMR-Spectra) \\
\hline 8 & d & f & f & $-\mathrm{CH}_{2}-: \delta^{13} \mathrm{C} \quad 26.2-25.2 \mathrm{ppm}$ (derive \\
\hline 9 & $\mathrm{c}$ & $\mathrm{e}$ & & from heteronucleic 2D-NMR-Spectra) \\
\hline 10 & c & e & $\mathrm{g}$ & $1.67-1.47$ und $1.43-$ \\
\hline 11 & d & f & & $1.26 \mathrm{ppm}$ \\
\hline 12 & $\mathrm{c}$ & e & & \\
\hline 13 & $\mathrm{c}$ & $\mathrm{e}$ & & \\
\hline 14 & 2.81 & f & & \\
\hline 15 & $\mathrm{c}$ & 127.0 & & \\
\hline 16 & 5.36 & 132.0 & & \\
\hline 17 & $2.078 \mathrm{~m}$ & 20.6 & & \\
\hline 18 & $0.976 \mathrm{t}(7.6)$ & 14.3 & & \\
\hline $\mathrm{OMe}$ & $3.695 \mathrm{~s}$ & 51.9 & & \\
\hline
\end{tabular}

Thus, with NMR analysis it was shown, that the double bonds in 18:5 were at position 3, 6, 9, 12 and 15.

\subsection{Biosynthesis of 18:5n-3 in P. parvum SAG 127.79}

To study the biosynthetic pathway of $18: 5 n-3$ of $P$. parvum, the alga was grown in the presence of various labelled FAs. As control P. parvum cultures without externally added FA were grown in parallel. The FAME profile was obtained by GC/MS to analyse the incorporation and metabolism of the labelled FAs in the algae. The rationale behind this experiment was to follow breakdown or extension/desaturation of FA precursors of 18:5n-3.

The first experiment was performed with $\left[1-{ }^{14} \mathrm{C}\right]-\mathrm{LA}$, to check whether the algal cells were able to take up and to incorporate FAs provided externally. The incorporation of $\left[1-{ }^{14} \mathrm{C}\right]-$ LA into cellular lipids was confirmed by RP-HPLC as shown in Fig. 16. In the control cultures no radioactive signal was detected whereas in the two other cultures ${ }^{14} \mathrm{C}$-labelled FAs were present. Some additional signals indicated the conversion of LA into other FAs. Signals of individual radio labelled FAs were superimposed in the elution profile. 


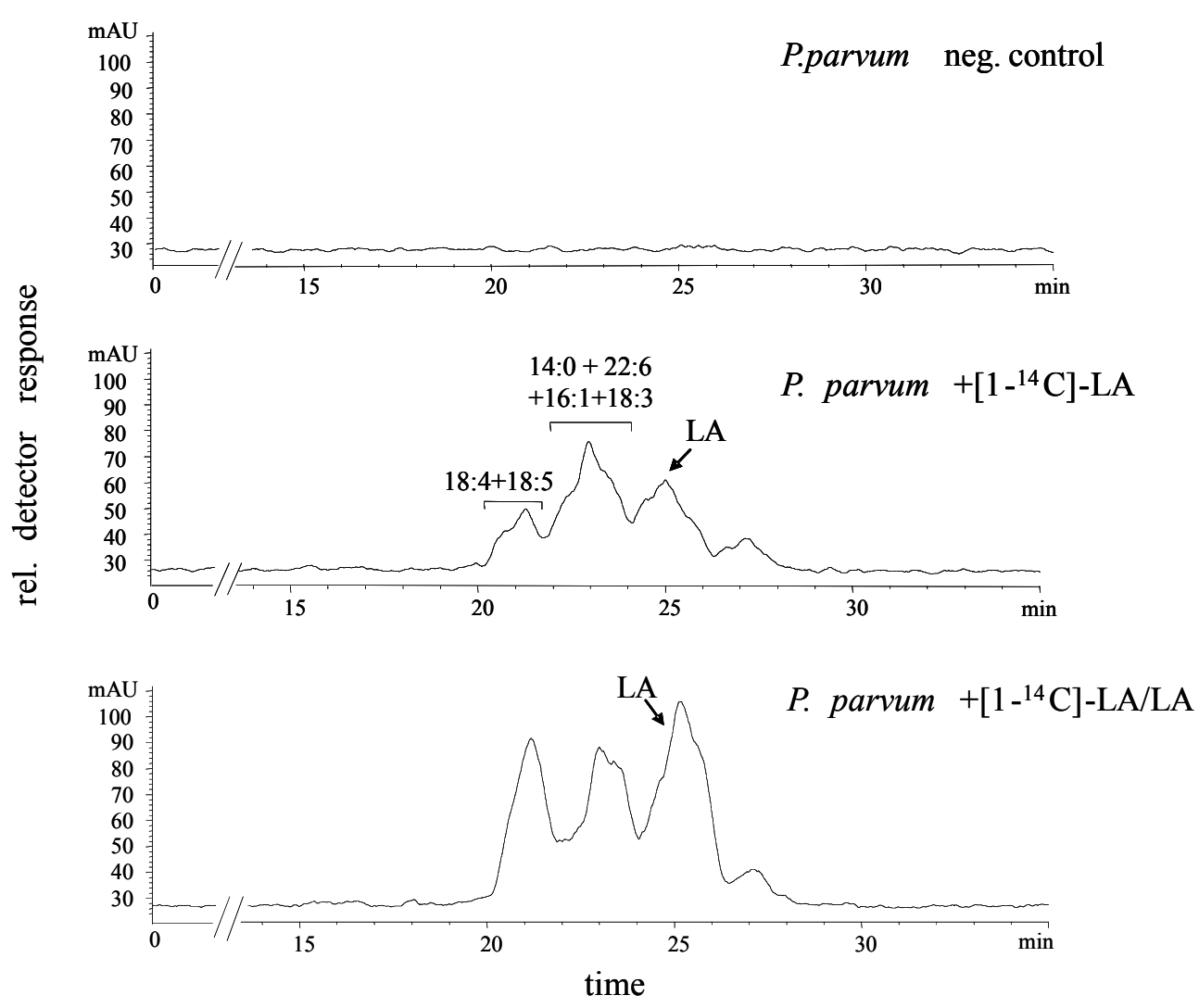

Figure 16 RP-HPLC analysis of feeding experiments with $\left[1-{ }^{14} \mathrm{C}\right]-\mathrm{LA}$. The chromatograms monitored by the radio detector are shown. $P$. parvum was incubated with either a mixture of $5.5 \mathrm{MBq}\left[1-{ }^{14} \mathrm{C}\right]-\mathrm{LA} / \mathrm{LA} 3: 1(\mathrm{v} / \mathrm{v})$ or just with $5.5 \mathrm{MBq}\left[1-{ }^{14} \mathrm{C}\right]-\mathrm{LA}$. As a negative control one culture was grown without externally added FA. These data represent one experiment.

Additional feeding experiments were performed with $\left[\mathrm{U}-{ }^{13} \mathrm{C}\right]-\mathrm{LA},\left[\mathrm{U}-{ }^{13} \mathrm{C}\right]-\mathrm{ALA}$ and $\left[19,19,20,20,20-D_{5}\right]-20: 5 n-3$, where the FAMEs were isolated via acidic hydrolysis which led to different distributions of FAME amounts in comparison to the previously obtained data in 2.1. Furthermore the algal cultures were harvested in different growth phases. For the feeding experiment the cultures were in the exponential phase, whereas the cultures of the FAME screen were harvested in the stationary phase.

Fig. 17A illustrates the relative proportions of FAs in P. parvum grown in the presence of $\left[\mathrm{U}_{-}{ }^{13} \mathrm{C}\right]-\mathrm{LA}$ and $\left[\mathrm{U}_{-}{ }^{13} \mathrm{C}\right]-\mathrm{ALA}$ in comparison to the control culture. In general, the relative amount of intracellular FAs clearly changed. In the control the main FAs were 18:1n-9 and 18:4n-3 followed by 18:5n-3. The supplementation of $\left[\mathrm{U}-{ }^{13} \mathrm{C}\right]-\mathrm{LA}$ and $\left[\mathrm{U}-{ }^{13} \mathrm{C}\right]-\mathrm{ALA}$ lead to an increase of LA and ALA, respectively, whereas the relative amount of all other FAs decreased.

P. parvum cells were also able to incorporate labelled EPA as shown in Fig. 17B. Again, the relative amounts of intracellular FAs changed in the supplemented cultures. The 
relative amount of EPA and 22:5n-3 increased, whereas the relative amount of e.g. LA and ALA decreased. In addition the relative amount of saturated and monounsaturated FAs 14:0, 16:0 and 18:1 (9Z) slightly increased in the supplemented cultures.

The incorporation and metabolism of labelled FAs was further analysed by extracting the GC chromatogram obtained for isotopes, which derived from labelled FAs. [U- $\left.{ }^{13} \mathrm{C}\right]-\mathrm{LA}$ and $\left[\mathrm{U}_{-}{ }^{13} \mathrm{C}\right]$-ALA were recovered from the mass spectra. Both FAs were further desaturated and elongated to $\left[\mathrm{U}_{-}{ }^{13} \mathrm{C}\right]-18: 4 \mathrm{n}-3$, $\left[\mathrm{U}-{ }^{13} \mathrm{C}\right]-18: 5 \mathrm{n}-3$ and $\left[\mathrm{U}_{-}{ }^{13} \mathrm{C}\right]-20: 5 \mathrm{n}-3$, as in the corresponding mass spectra the appropriate increased mass fragments were found (Fig. 17A). The signals, which appeared in the chromatogram after extracting certain isotopes (e.g. $m / z 312$ for $\left[\mathrm{U}_{-}{ }^{13} \mathrm{C}\right]-\mathrm{LA}$ and $\mathrm{m} / \mathrm{z} 294$ for LA), were integrated and the distribution of labelled and unlabelled FAMEs were calculated. The comparison of unlabelled and labelled LA, ALA, 18:4n-3, 18:5n-3 and 20:5n-3 in one P. parvum culture, supplemented with $\left[\mathrm{U}^{-13} \mathrm{C}\right]-\mathrm{LA}$ is shown in Fig. 18A. In addition the mass spectra of $\left[\mathrm{U}_{-}{ }^{13} \mathrm{C}\right]-\mathrm{LA}$ and $\left[\mathrm{U}_{-}{ }^{13} \mathrm{C}\right]-18: 4 \mathrm{n}-4$ are shown. LA, ALA and EPA showed a high degree of labelling (94\% of $\left[\mathrm{U}_{-}{ }^{13} \mathrm{C}\right]-\mathrm{LA}, 96 \%$ of $\left[\mathrm{U}_{-}{ }^{13} \mathrm{C}\right]-\mathrm{ALA}$ and $\left.96 \%\left[\mathrm{U}-{ }^{13} \mathrm{C}\right]-20: 5 \mathrm{n}-3\right)$, whereas the proportion of labelled and unlabelled 18:4n-3 and 18:5n-3 was similar with $48 \%$ of $\left[\mathrm{U}-{ }^{13} \mathrm{C}\right]-18: 4 \mathrm{n}-3$ and $41 \%$ of $\left[\mathrm{U}-{ }^{13} \mathrm{C}\right]-18: 5 \mathrm{n}-3$. 


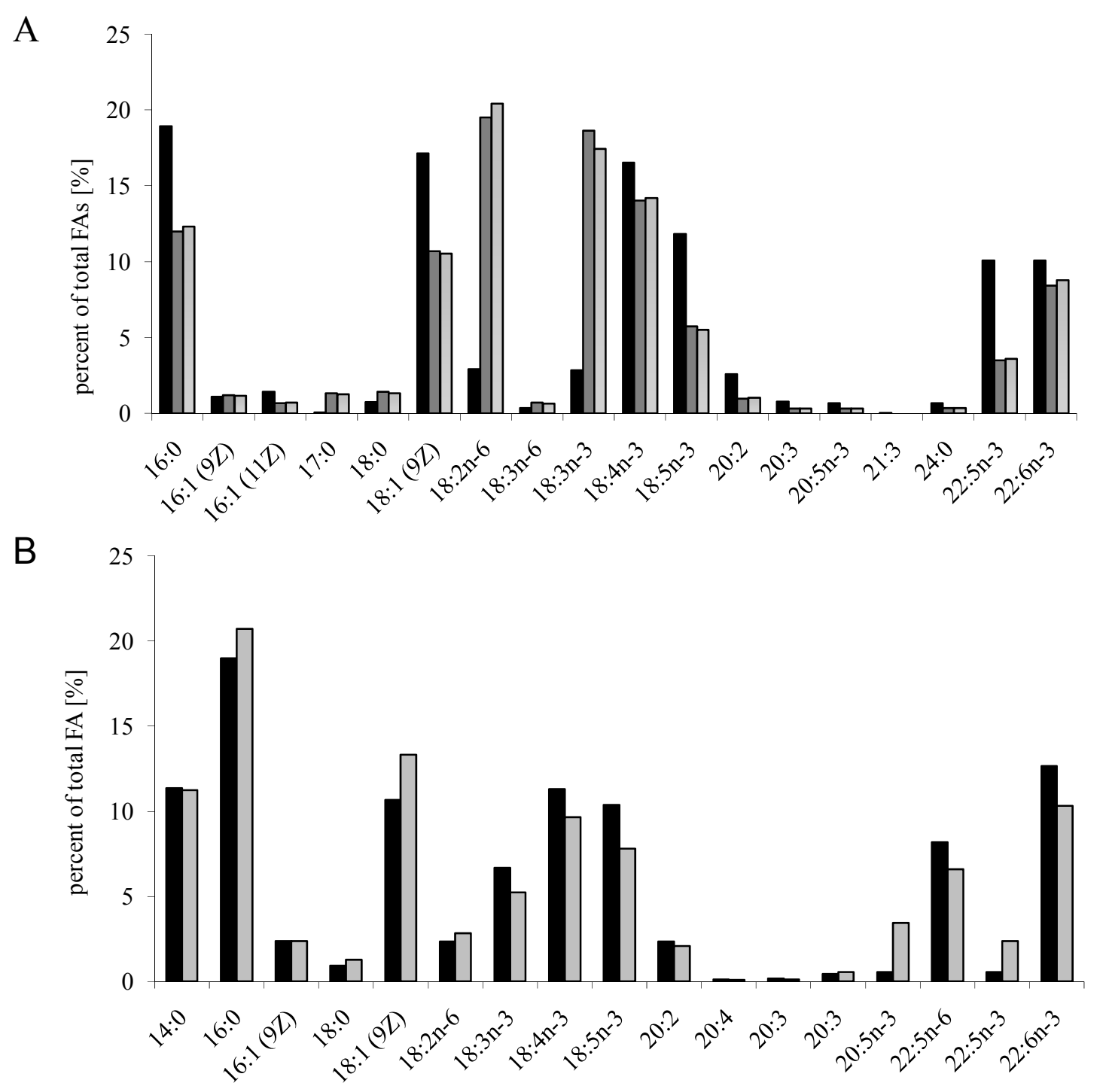

Figure 17 Relative proportion of FAMEs within three $P$. parvum SAG 127.79 cultures grown with and without labelled FAs. A) $\left[\mathrm{U}_{-}{ }^{13} \mathrm{C}\right] \mathrm{LA}$ and $\left[\mathrm{U}_{-}{ }^{13} \mathrm{C}\right] \mathrm{ALA}$ and $\left.\mathrm{B}\right)$ $\left[19,19,20,20,20-D_{5}\right]-20: 5 n-3$. FAMEs were analyzed via GC/MS. Black bars represent negative control, dark grey bars represent cultures incubated with ${ }^{13} \mathrm{C}$-LA and bright grey bars cultures either incubated with A) ${ }^{13} \mathrm{C}$-ALA or B) $\mathrm{D}_{5}-20: 5 n-3$. These graphics show one representative out of three experiments.

The feeding experiment with deuterated EPA demonstrated that $\mathrm{D}_{5}$-EPA was further desaturated and elongated to 22:5n-3 and DHA. Labelled $D_{5}-18: 5 n-3$ was not detected, indicating, that $18: 5 n-3$ is not synthesised by $\beta$-oxidation of EPA. The distribution of labelled and non labelled FAs is illustrated in Fig. $18 \mathrm{~B}$. The mass spectra represent $\mathrm{D}_{5^{-}}$ 
20:5n-3 and $D_{5}-22: 5 n-3$. The major amount of $20: 5 n-3$ and $22: 5 n-3$ of the $P$. parvum culture was labelled (93\% $\mathrm{D}_{5}-20: 5 \mathrm{n}-3$ and $\left.\mathrm{D}_{5}-22: 5 \mathrm{n}-3\right)$.

Taken together, the results indicate, that $18: 5^{(3 Z, 5 Z, 9 Z, 12 Z, 15 Z)}$ is synthesised by the activity of an $\Delta 3$-desaturase on 18:4n-3 and not via $\beta$-oxidation of $20: 5 n-3$.

A
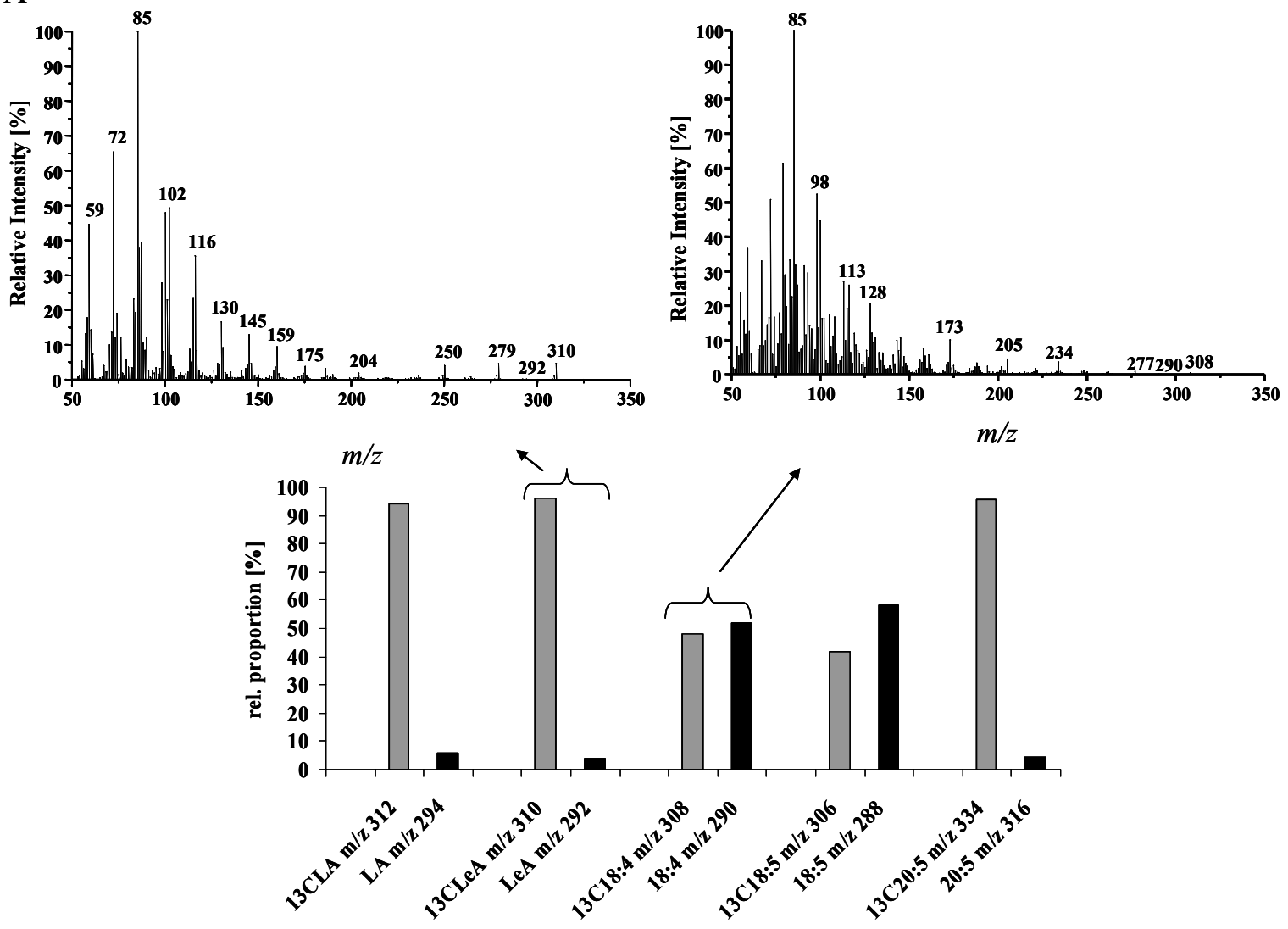


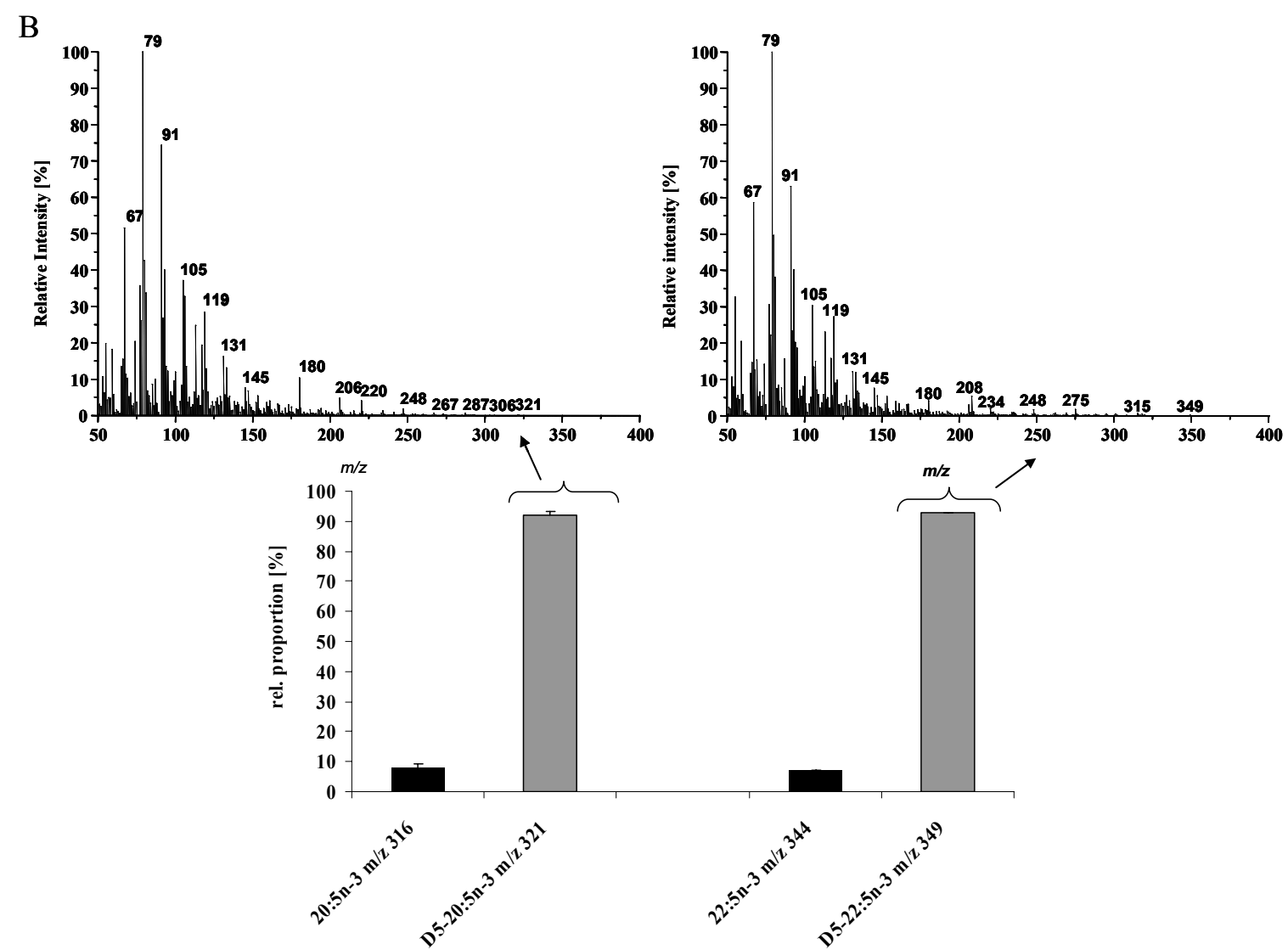

Figure 18. GC/MS analysis of FAMEs of $P$. parvum supplemented with labelled FAs. A, $\left[\mathrm{U}_{-}{ }^{13} \mathrm{C}\right]-\mathrm{LA} ; \mathrm{B},\left[19,19,20,20,20-\mathrm{D}_{5}\right]-20: 5 \mathrm{n}-3$. The chromatograms were scanned for the mass ions with the following $\mathrm{m} / \mathrm{z}$ : A, LA: 294 ; $\left[\mathrm{U}-{ }^{13} \mathrm{C}\right]-\mathrm{LA}: 312$, ALA: 292 , $\left[\mathrm{U}-{ }^{13} \mathrm{C}\right]-\mathrm{ALA}$ : 310, 18:4n-3: 290, [U- $\left.{ }^{13} \mathrm{C}\right]-18: 4 n-3: 308,18: 5 n-3: 219$, [U- $\left.{ }^{13} \mathrm{C}\right]-18: 5 n-3: 232,20: 5 n-3: 316$ and $\left[\mathrm{U}_{-}{ }^{13} \mathrm{C}\right]-20: 5 \mathrm{n}-3: 334 ; \mathbf{B}, 20: 5 \mathrm{n}-3: 201, \mathrm{D}_{5}-20: 5 \mathrm{n}-3: 206,22: 5 \mathrm{n}-3: 201, \mathrm{D}_{5}-22: 5 \mathrm{n}-3$ : 206, 22:6n-3:255, D5-22:6n-3: 260. Representative data of one out of two independent experiments are shown. 
In addition to the FAME screening (chapter III-1) it was another aim of this work to identify oxylipins in all the SAG microalgal strains. The first screening of oxylipins was done with cyanobacteria, but without reliable putative identification (III-1.3). Therefore, oxylipin producing microalgae were identified by an alternative approach as described in the following section.

\section{Lipoxygenase pathway of selected cyanobacterial strains}

Genomic sequences of different microalgae were searched resembling enzymes catalysing the synthesis of oxylipins. The main focus was placed on LOXs, which catalyse the oxidation of FAs to FA hydroxyperoxides. The gene databases (NCBI, DOE Joint-Genome Institute (http://genome.jgi-psf.org/)) were screened for LOX sequences of algae. Hitherto only a few algal genomes are published and out of these only four encode putative lipoxygenases. Although it is clear that the restricted search in published genomes of microalgae cannot replace a complete screen of microalgal strains as originally planned, three novel LOX sequences of cyanobacteria and one of the green alga Chlamydomonas reinhardtii were identified. In the following the isolation and characterisation of the three LOXs identified for cyanobacteria is described.

\subsection{Phylogenetic analysis of new lipoxygenases from cyanobacteria}

Database searches and alignments of LOX amino acid sequences against the publically available genomes of microalgae yielded two putative LOXs from the cyanobacterium Nostoc punctiforme PCC73102 and a peroxidase-LOX fusion protein (Acc.no. NP_478445) from cyanobacterium Nostoc sp PCC 7120, which was named NspFP. The two LOXs of N. punctiforme were named NpLOX1 (Acc. no. ZP_00106490) and NpLOX2 (Acc. no. ZP_00107030), respectively. The amino acid sequence of NpLOX1 shared highest identity (37\%) to the LOX from Pseudomonas aeruginosa (Vidal-Mas, 2005) and the corresponding sequence of NpLOX2 showed highest identity (38\%) to NpLOX1. The amino acid sequence of the fusion protein had highest identity (34\%) to a naturally occurring AOS-LOX fusion protein from the coral Plexaura homomalla (Koljak et al. 1997). For NspFP amino acid sequence comparisons were performed for the two domains individually. The peroxidase domain had highest identity (49\%) to a protein with unknown function from Oryza sativa (Acc. no. XP 472850). The LOX-coding domain 
showed the highest identity (38 \%) to a hypothetical protein from Myxococcus xanthus DK 1622 (Acc. no. ABF86480).

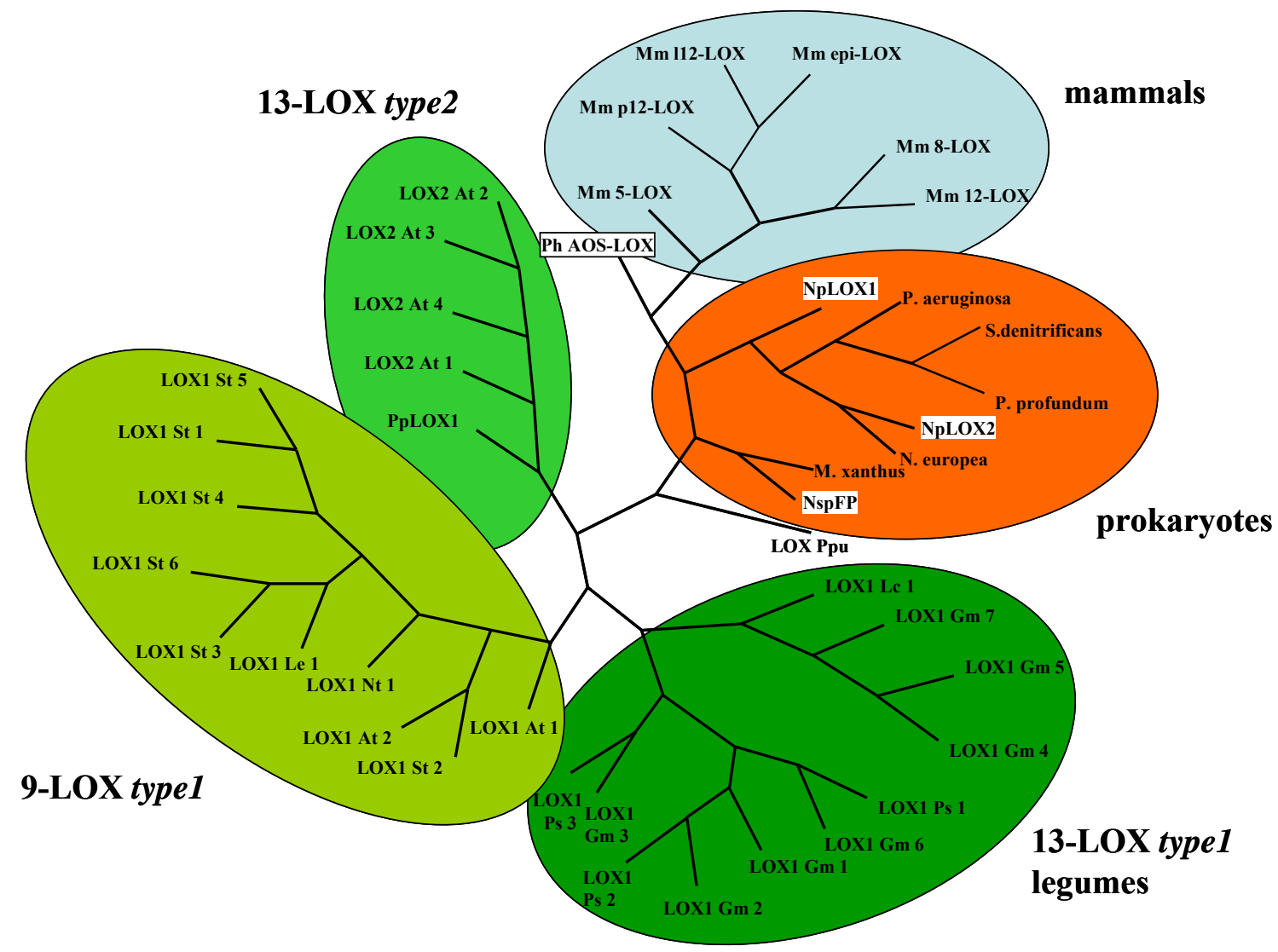

Figure 19 Comparative phylogenetic analysis of NpLOX1, NpLOX2, NspFP and selective LOXs from mammals, plants, coral, algae and bacteria, using phylip 3.5.

Acc.no.: Mus musculus, 5-LOX AAC37673; 8-LOX CAA75003; 12-LOX CAA74714; 112-LOX AAA20658; p12-LOX AAA20659; epi-LOX NP663717; P.homomalla AOSLOX O16025; Arabidopsis thaliana type 2 LOX, At1 Q06327; At2 CAB56692; At3 CAC19364; At4 CAG38328; Physcomitrella patens, PpLOX1 CAE47464; Solanum tuberosum, LOX1 St1 CAA5572; LOX1 St2 AAD09202; LOX1 St3 AAB31252; LOX1 St4 CAA64766; LOX1 St5 CAA64765; LOX1 St6 AAB67860; A. thaliana type1 9-LOX, At1 NP175900, At2 NP188879, Lycopersicon esculentum Le1 P38415; Nicotiana tabacum LOX1 Nt1 CAA58859; Glycine max, Gm1 CAA47717; Gm2 P09439; Gm3 CAA31664; Gm4 P38417; Gm5 AAB67732; Gm6 AAA96817; Gm7 AAC49159; Pisum sativum, Ps1 AAB71759; Ps2 CAA55318; Lens culinaris Lc1 CAA50483; Porphyra purpureum, Ppu AAA61791; P. aeruginosa, AF479686; Nitrosomonas europaea, BX321860; Shewanella denitrificans, OS-217:Q3P217; Photobacterium profundum, 3TCK: ZP_01218321, M. xanthus DK 1622 hypothetical protein MXAN_1745 YP_629995. N. punctiforme: NpLOX1 ZP_00106490; NPLOX2 ZP_00107030, N. sp. SAG 25.82: NspFP NP_478445. 
Results

Phylogenetic analysis of NpLOX1, NpLOX2 and NspFP with selected LOX sequences from plants, mammals, algae, corals, mosses and bacteria showed that the cyanobacterial sequences form a separate clade together with other mostly putative prokaryotic LOX sequences (Fig. 19). Interestingly, the fusion protein of Nostoc sp PCC 7120 falls into a separate group with M. xanthus, whereas NpLOX1 and 2 were closer related to other prokaryotic sequences. Furthermore, the clade of the bacterial LOXs was closer related to that of mammalian and eukaryotic algal LOXs than to sequences from plants. 


\subsection{Oxylipin formation in N. punctiforme PCC 73102}

\subsubsection{Amino acid sequence alignment with other LOX sequences}

To obtain more information on the biochemical features of NpLOX1 and 2 their protein sequences were aligned with other LOXs from plant, moss, coral and $P$. aeruginosa (Fig. 20) that have previously been characterised biochemically. Sequence similarity is mainly found around the active site residues of the proteins (Liavonchanka and Feussner 2006). The central histidine-rich region, including three histidines (His-327, His-332, His510 in NpLOX1) which constitute part of the iron binding site (Siedow 1991), are conserved in all six LOX-sequences. Two additional amino acids, asparagine (Asn-514 in NpLOX1) and isoleucine (Ile-630 in NpLOX1) may be also involved in the iron binding and are conserved in NpLOX1. For NpLOX2 a C-terminal valine (Val-548) corresponds to the position of Ile-630 in NpLOX1. At the position of the three determinants involved in substrate- and regiospecificity (Sloane et al. 1991; Borngräber et al. 1996a; Hornung et al. 1999b) both NpLOXs harbour amino acids different from those of the other four LOX sequences. Particularly, NpLOX1 and NpLOX2 sequences harbour two leucine residues (Leu-384 and Leu-385) at the Sloane site instead of methionine (Met-434) and phenylalanine (Phe-435) in PaLOX13 or cysteine (Cys-643) and phenylalanine (Phe-644) in AtLOX3. However, the residue determining the stereospecificity of LOXs (Coffa site) is an alanine residue in both sequences, suggesting both LOXs to be $S$-specific LOXs (Coffa and Brash 2004).

To isolate the coding sequences for the two cyanobacterial LOX isoforms, gene specific primers were used to amplify the two fragments from genomic DNA. Genomic DNA can serve as template, because prokaryotic genes do not contain introns. The obtained fragment of NpLOX1 contains a complete open reading frame of $1893 \mathrm{bp}$ encoding a protein of 630 amino acids with a molecular weight of $70.9 \mathrm{k}$. The open reading frame of NpLOX2 had a length of $1647 \mathrm{bp}$ encoding a protein of 548 amino acids with a molecular weight of $62.9 \mathrm{k}$. 

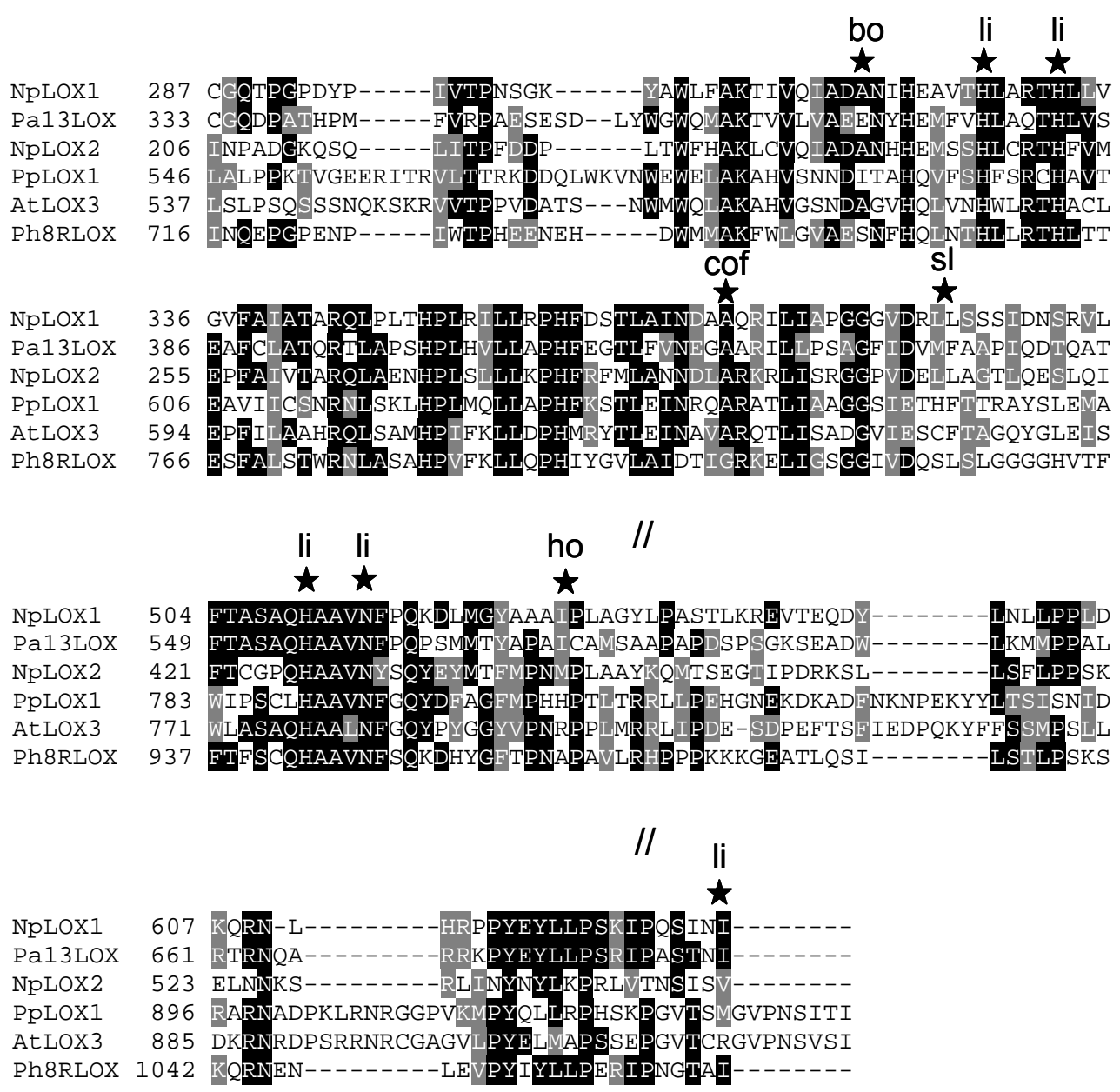

Figure 20. Partially deduced amino acid sequence alignment of NpLOX1 and NpLOX2 in comparison with AtLOX3 (Acc. no. AAF79461) from A. thaliana, PpLOX1 (Acc. no. CAE47464) from P. patens, Pa-LOX (Acc. no. AAL85880) from P. aeruginosa and Ph8R-LOX (Acc. no. AAC47283) from the coral P. homomalla. For alignment, the MultAlin program was used (Corpet 1988). The following conserved amino acids are marked with a star: the residues involved in iron ligation (three histidines, an asparagine and an isoleucine; $l i$ ), and the three determinants for substrate and regiospecificity (bo, according to Borngräber et al.(1996), sl, according to Sloane et al.(1992), ho, according to Hornung et al.(1999) ) 


\subsubsection{Characterisation of in vitro LOX activity of NpLOX1 and NpLOX2}

For biochemical characterisation NpLOX1 and NpLOX2 ORFs were subcloned into the pEXP5-NT/TOPO ${ }^{\circledR}$ expression vector and heterologously expressed in E. coli BL21(DE3) cells (8.3). Crude cell extracts were prepared and incubated with several FA substrates for LOX activity tests as described in section 8.7 and 9.3. Initial analysis showed that both enzymes, NpLOX1 and NpLOX2, respectively, converted C18- and C20-PUFAs to the corresponding hydroperoxides and that oxygen was inserted at the $\omega-6$ position. The analysis of products formed showed the conversion of LA into (9Z,11E,13S)-13hydro(pero)xy-octadeca-9,11-dienoic acid (13-H(P)ODE) that was detected after reduction as hydroxy FAs as shown in Fig. 21 for both LOXs. Due to the similar reaction characteristics, which were determined during these experiments and the same active site determinants in both enzymes (compare Fig. 20), no major differences between both enzymes were expected. Thus, NpLOX1 was analysed in more detail first.

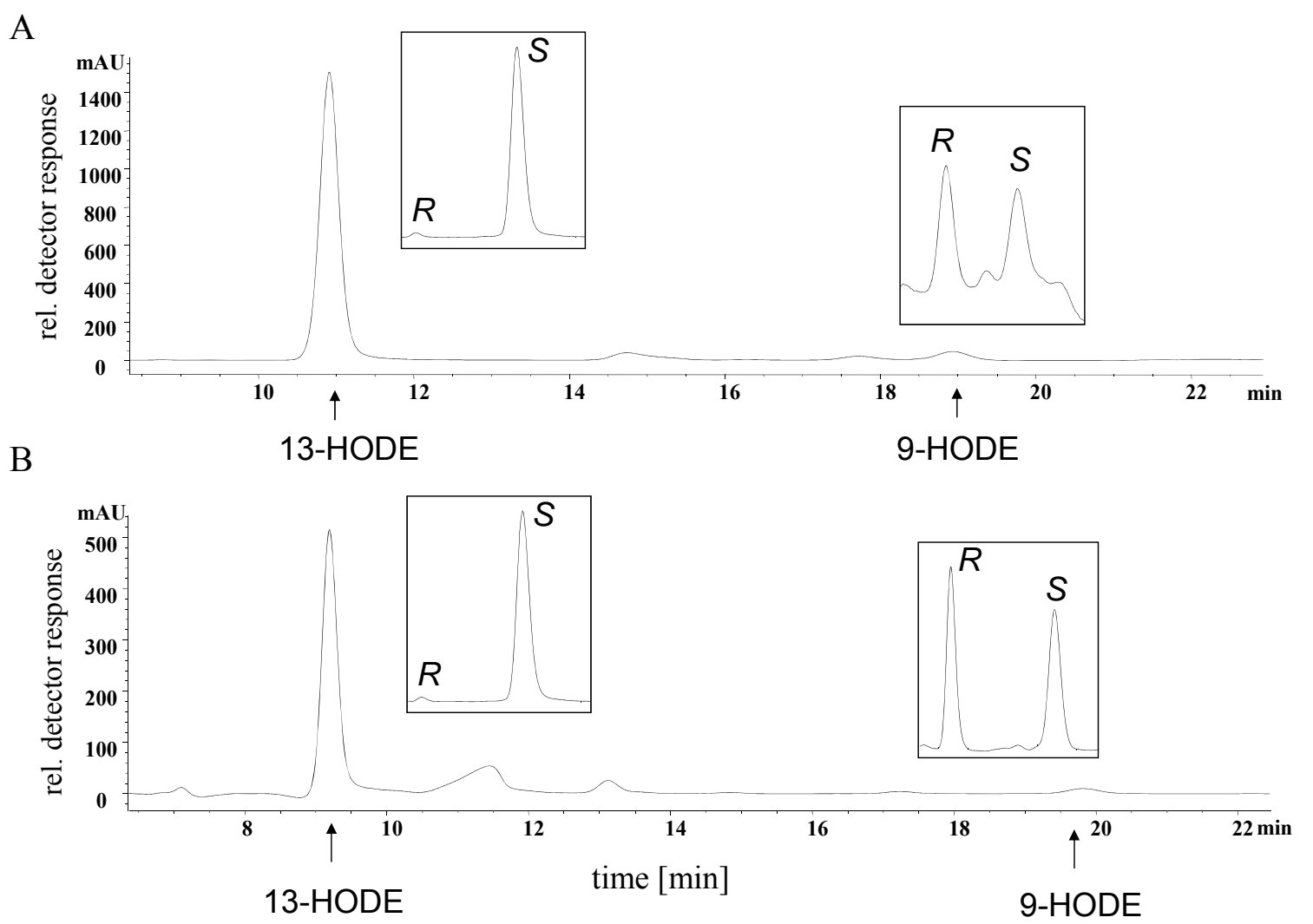

Figure 21. HPLC analysis of products formed by NpLOX1 and NpLOX2 with LA.

A, NpLOX1, B, NpLOX2. Conversion of LA was performed in 50mM Tris/HCL, pH 8.0. Products were reduced with $50 \mathrm{mM} \mathrm{SnCl}_{2}$. Insets show the formation of $R$ - and $S$ enantiomer as determined by CP-HPLC analysis. Chromatogram represent one experiment out of ten experiments. 
The pH-optimum for the conversion of LA by NpLOX1 was determined by summing up the integrals of all isomeric hydroxy FA products determined by HPLC analysis at $\mathrm{pH}$ amounts ranging from $\mathrm{pH} 4.5$ to $\mathrm{pH} 12.0$ (bars in Fig. 22). The $\mathrm{pH}$ optimum was broad, ranging from $\mathrm{pH} 4.5$ to $\mathrm{pH} 8.5$ with a maximum at $\mathrm{pH}$ 8.0. 13-H(P)ODE, (13-H(P)OTE), and $(6 Z, 9 Z, 11 E, 13 S)$-13-hydro(pero)xy-6,9,11-octadecatrienoic acid (13 $\gamma$-H(P)OTE) were preferred products after addition of LA, ALA and GLA, respectively. AA was predominantly converted to $(5 Z, 8 Z, 11 Z, 13 E, 15 \mathrm{~S})-15$-hydro(pero)xy-5,8,11,13eicosatetraenoic acid (15-H(P)ETE). CP-HPLC analysis of the enantiomers showed that in all cases formation of the $S$-enantiomers dominated. In Table 14 the relative amounts of the hydro(pero)xides and the $S$-enantiomers are given in percentages. For the absolute activity the highest regiospecificity of NpLOX1 was observed at pH 8.0 (92\% of 13-H(P)ODE; Fig. 22, lines). However, the highest stereospecificity was measured at $\mathrm{pH} 6.0$, with a relative amount of about $99 \%$ of the $S$-enantiomer of $13-\mathrm{H}(\mathrm{P}) \mathrm{ODE}$. In summary, the analysis of the $13-\mathrm{H}(\mathrm{P}) \mathrm{ODE}$ enantiomers revealed that more than $80 \%$ of them occurred as $S$-enantiomers at all $\mathrm{pH}$ values tested. Furthermore, the data show, that 9-H(P)ODE was mainly a product of chemical oxidation at all $\mathrm{pH}$ values tested, because its enantiomer ratio was almost racemic.

Table 14 FA hydroperoxides formed by NpLOX1 in vitro with different FA substrates. Reactions were carried out at $\mathrm{pH}$ 8.0. Values represent the mean of two independent experiments \pm standard deviation.

\begin{tabular}{l|lllll}
\hline \multicolumn{5}{l}{ Substrates } & \multicolumn{5}{c}{ Products } \\
\hline Arachidonic acid & 15 -HETE & 12 -HETE & 11 -HETE & 8 -HETE & 5-HETE \\
Total hydroperoxides ( \%) & $74 \pm 10$ & $1,6 \pm 0,2$ & $19,2 \pm 14$ & $4,4 \pm 3,9$ & n.d. \\
$S$-Enantiomer (\%) & $97,4 \pm 1,2$ & $54,4 \pm 9,1$ & $77 \pm 12,5$ & $48,1 \pm 1,9$ & \\
-Linolenic acid & $13 \gamma$-HOTE & $10 \gamma$-HOTE & $9 \gamma$-HOTE & $6 \gamma$-HOTE & \\
Total hydroperoxides ( \%) & $65 \pm 16$ & $4,23 \pm 1,2$ & $6,2 \pm 1,2$ & $23,5 \pm 15$ & \\
$S$-Enantiomer (\%) & $99,3 \pm 0,44$ & $60,5 \pm 4,1$ & $62,1 \pm 13,9$ & $63,1 \pm 6,9$ & \\
$\boldsymbol{\alpha}$-Linolenic acid & 16 -HOTE & 13 -HOTE & $12-\mathrm{HOTE}$ & 9 -HOTE & \\
Total hydroperoxides ( \%) & $6,57 \pm 5.42$ & $81,2 \pm 13,1$ & $5,76 \pm 4.63$ & $6,5 \pm 3,6$ & \\
$S$-Enantiomer (\%) & $50 \pm 3.5$ & $90,2 \pm 12$ & $48 \pm 2,2$ & $51 \pm 2,8$ & \\
Linoleic acid & 13 -HODE & $9-\mathrm{HODE}$ & & & \\
Total hydroperoxides ( \%) & $94,3 \pm 2,7$ & $5,7 \pm 2,7$ & & & \\
$S$-Enantiomer (\%) & $98,4 \pm 0,2$ & $60,2 \pm 6,7$ & & & \\
\hline
\end{tabular}




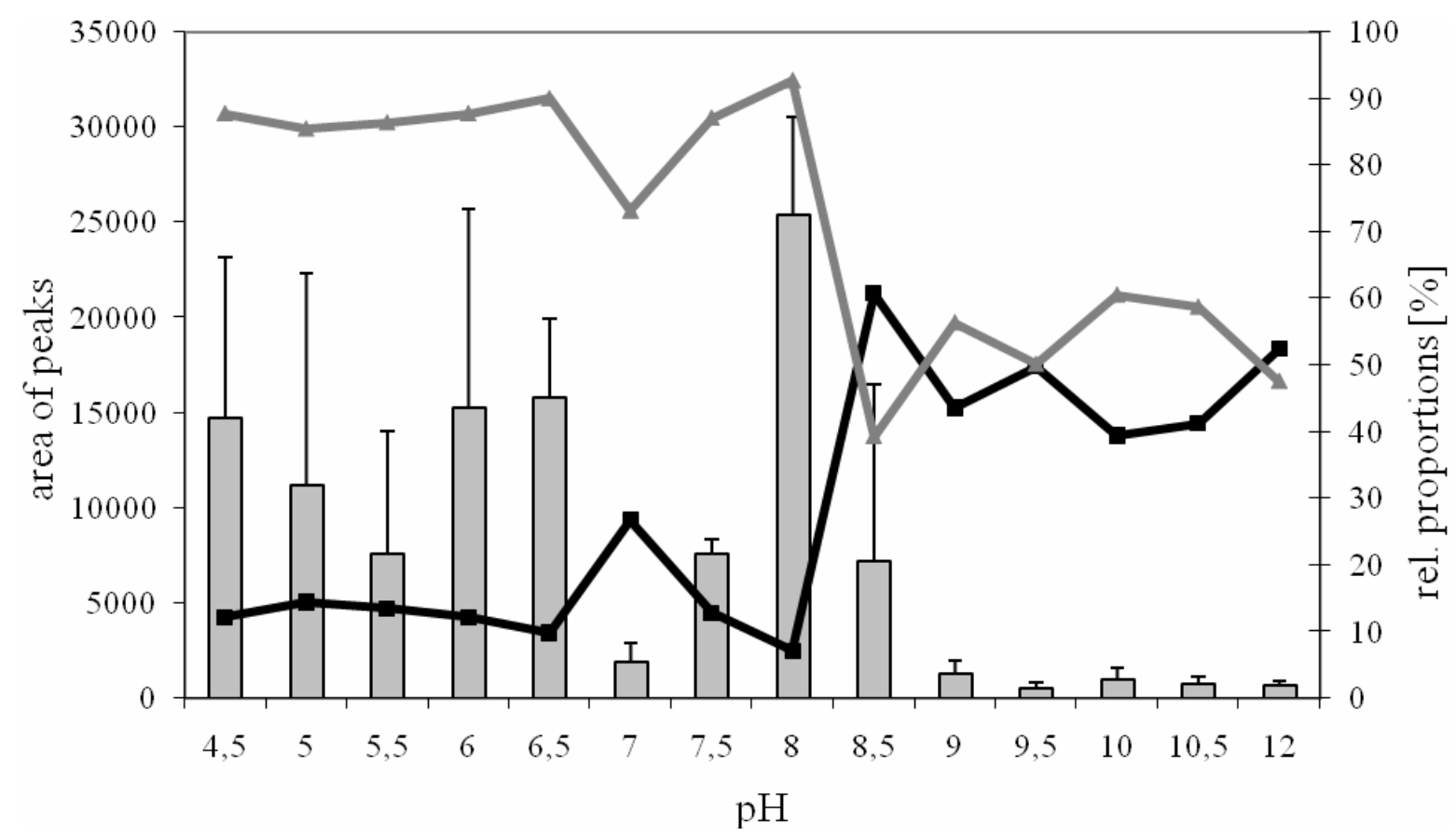

Figure 22. Production of 13-HODE and regiospecificity of NpLOX1 in dependence of different pH-values. The grey bars represent the amounts of 13-HODE produced by NpLOX1. The regiospecificity is presented by the percentages of 9-HODE (black curve) and 13-HODE (grey curve). Mean values represent three independent experiments.

The substrate preference of NpLOX1 was determined at $\mathrm{pH} 8.0$ by incubating an equimolar mixture of the four different FAs for 30 minutes with the enzyme extract. Afterwards the reaction was stopped and the consumption of the substrates determined. However, the enzyme showed no substrate preference (data not shown).

To address loss of activity during the characterisation of NpLOX2 in detail, SDS-PAGE and Western blot (data not shown) analyses were performed. The production of NpLOX2 in different expression cell lines was verified first by SDS-PAGE. The supernatant and pellet were analysed separately. In order to compare the solubility of the expressed protein under different conditions, half of the expression cultures were grown in presence of $6 \%$ Ethanol. The expression was successful in Rosetta and BL21 cells, whereas no protein band was visible for the expression in the other tested cell lines including BL21star, RP and HMS74 (Fig. 23). Protein activity was found in the pellet only and not in the supernatant, indicating insolubility of the protein. The addition of $6 \%$ ethanol during the expression did not increase the yield of soluble protein. 


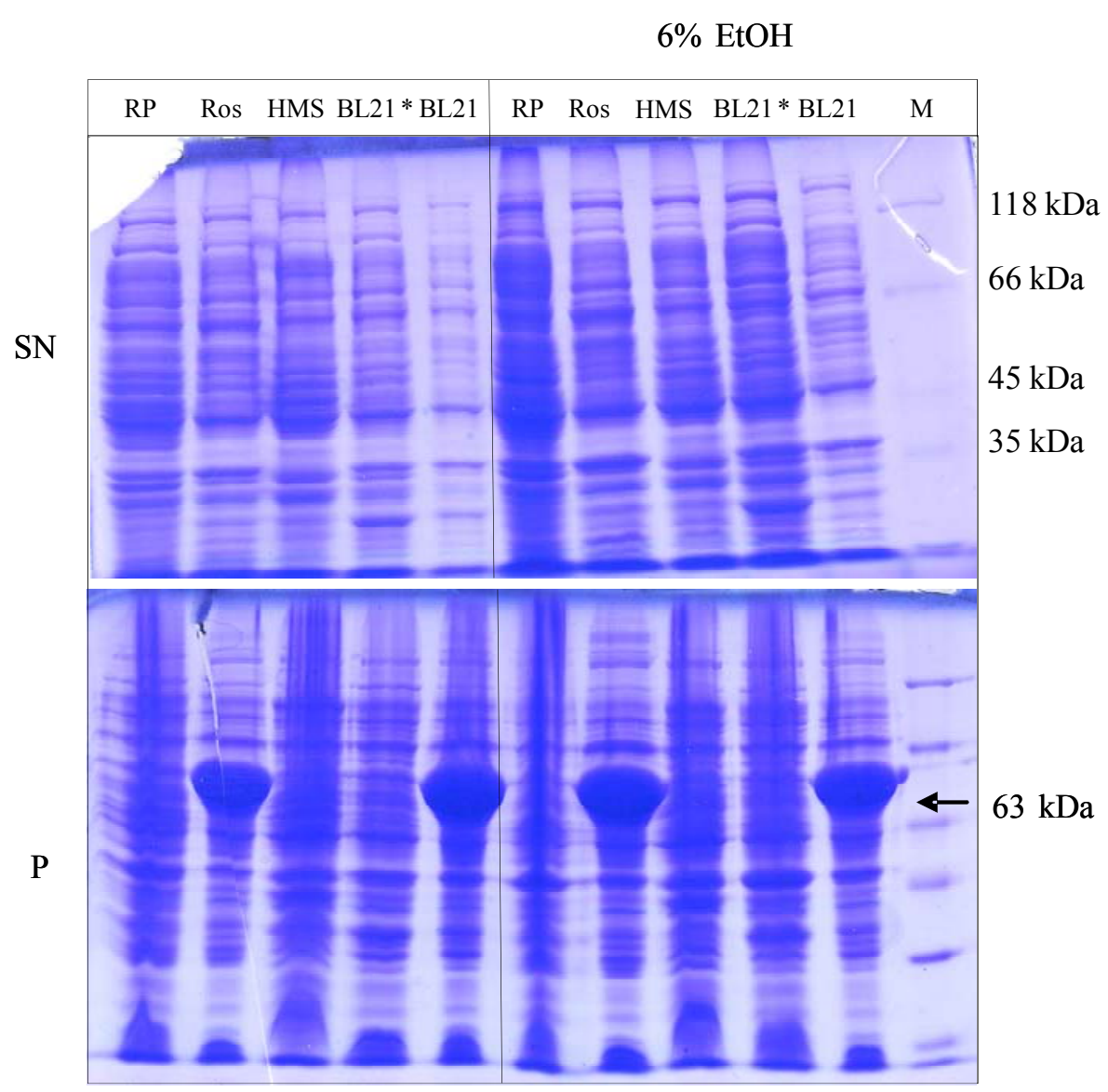

Figure 23 SDS-PAGE of NpLOX2 in pEXP5-NT expressed in different E. coli expression strains. The cells were either grown without or in presence of $6 \%$ ethanol. Expression was performed overnight at $16^{\circ} \mathrm{C}, 3 \mu \mathrm{l}$ of supernatant $(\mathrm{SN})$ and pellet $(\mathrm{P})$ fraction were loaded on a $10 \%$ SDS gel. The experiment was performed once.

\subsubsection{LOX activity in vivo}

One possible role of 13-LOXs is their involvement in plants wound response (Schilmiller and Howe 2005b). As products of the LOX may occur in wounded and non-wounded cyanobacteria both states were compared. Therefore half of the harvested cell material was wounded by sonification as described before for diatoms and mosses (Pohnert 2000; Wichard et al. 2005). An aliquot of the cell material wounded was transferred into fresh BG11 medium to test, whether the cells survived sonification or died. The cultures grew normally and had the characteristic blue green colour.

Both, the wounded and non-wounded material was extracted and the oxylipin containing fractions, free and esterified oxylipins as well as hydro(pero)xy dienoic and trienoic FAs 
were analysed. The amount of free 13-hydroxy dienoic and trienoic FAs was remarkably higher in cells which have been wounded (13-HODE: $0.13 \mathrm{nmol} / \mathrm{g}$ f.w. vs. $7.3 \mathrm{nmol} / \mathrm{g} \mathrm{f.w.;}$ 13-HOTE: $0.07 \mathrm{nmol} / \mathrm{g}$ f.w. vs. $4.29 \mathrm{nmol} / \mathrm{g}$ f.w.) whereas no remarkable differences in the amounts of 9-hydroxy dienoic and trienoic FAs were observed between wounded and nonwounded cells (Fig. 24A). Chiral analysis of the identified substances showed, that only 13-HODE and 13-HOTE were products of LOX activity whereas the detected 9-HODE and 9-HOTE originated from autoxidation (insets in Fig. 24A). For the esterified oxylipins the obtained data deviated from that of the free oxylipins. The amount of esterified hydroxy dienoic FAs were close to detection limit, especially for the non-wounded cultures. The amount of esterified oxylipins slightly increased after wounding (13HODEme: $0.16 \mathrm{nmol} / \mathrm{g}$ f.w. vs. $0.25 \mathrm{nmol} / \mathrm{g}$ f.w.; 13 -HOTEme: $0.10 \mathrm{nmol} / \mathrm{g}$ f.w. vs. $0.14 \mathrm{nmol} / \mathrm{g}$ f.w.) (Fig. 24B). Chirality of the esterified 13-HODE/HOTE corresponded to that of the free oxylipin products of LOX activity whereas 9-HODEme/HOTEme as well as 12- and 16-HOTEme seemed to derive from chemical autoxidation (Insets in Fig. 24B). Further oxylipins like C16- or C20-PUFA-derived hydroxides were detected, neither in non-wounded nor in wounded cyanobacteria. In addition FA hydroperoxides, jasmonic acid and 12-oxo phytodienoic acid were below the detection limit. 
A

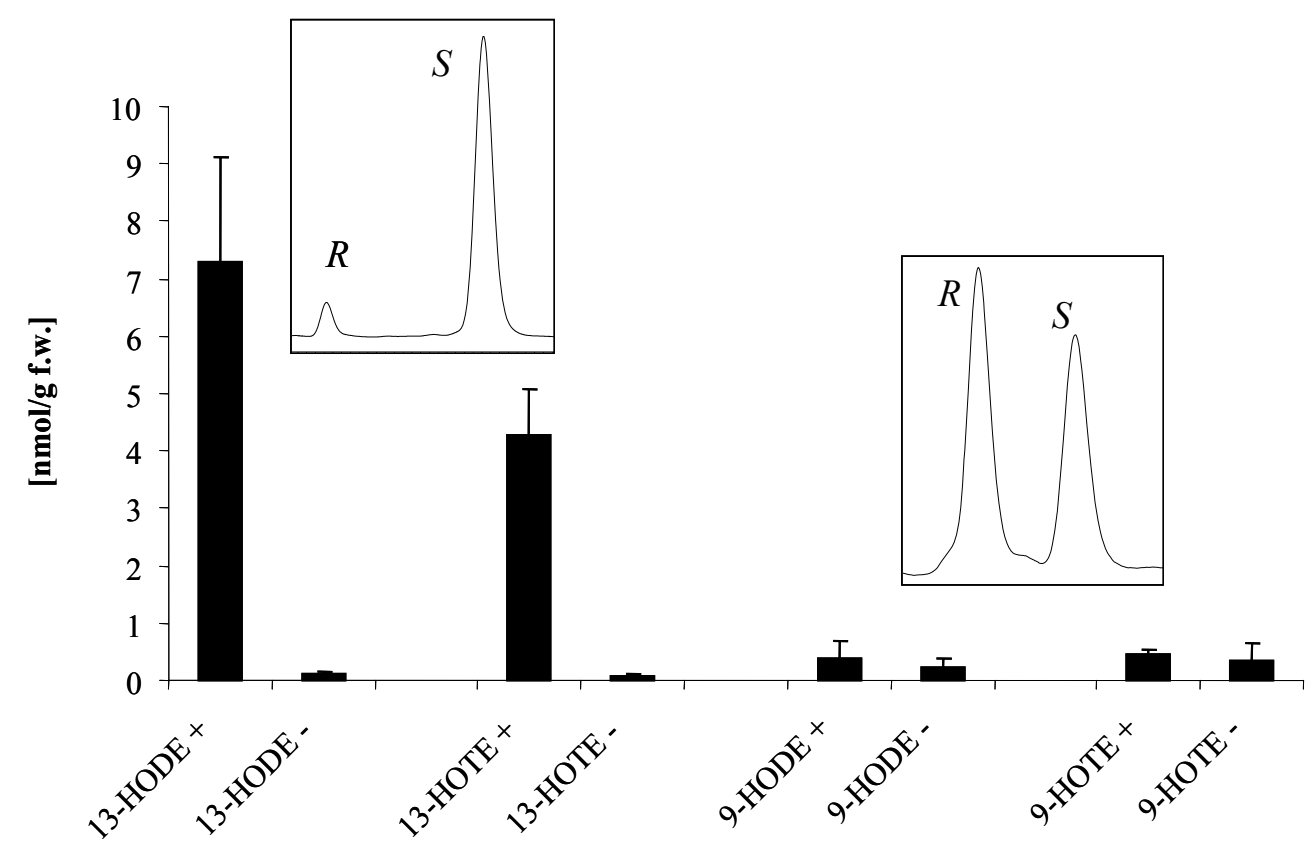

B

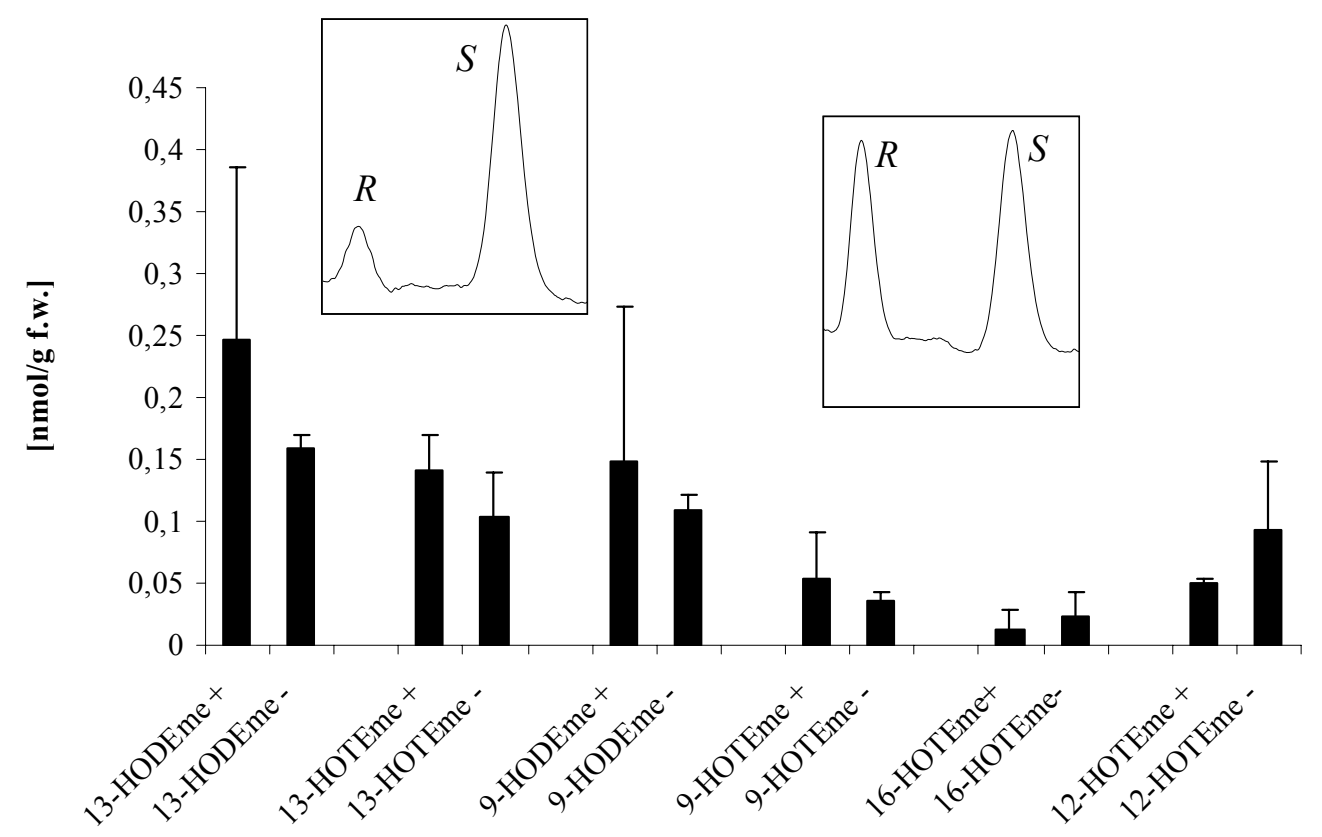

Figure 24. Formation of oxylipins upon wounding of $\boldsymbol{N}$. punctiforme. A, non-esterified hydroxy FAs (HODE and HOTE) and B, esterified hydroxyl FAs (HODEme and HOTEme). The bars represent the amounts of detected hydroxy FAs from non-wounded (-) or wounded $(+)$ cyanobacteria. Each data point represents an average of two independent experiments, standard deviation is given. The insets show the CP-HPLC analyses for 13HODE and 13-HODEme respectively. 


\subsection{Characterisation of a peroxidase-LOX fusion protein from Nostoc sp.}

\subsubsection{Alignment of individual peroxidase and LOX domains of the fusion protein with related sequences}

A comparison of the deduced amino acid sequence alignment of the peroxidase coding domain with other peroxidase-like sequences from $O$. sativa, the coral P. homomalla and the bacterium Sulfitobacter sp. EE-36 showed that certain amino acids are conserved which could either be involved in heme binding or present catalytic residues of the active site of a peroxidase. The crystal structure of the AOS domain in the coral (Oldham et al. 2005) revealed, that three residues are important for the distal face of the heme group: Tyr66, His-67 and Asn-137; the proximal-side residues are Arg-349 and Tyr-353. In the peroxidase domain of the cyanobacterial fusion protein the residues for the distal face of the heme group could be identified as His-41, Tyr-42 and Asn-118. (Fig. 25A, asterisks). In contrast, the residues on the proximal face of the heme group differ from that of the coral. Instead of an arginine (Arg-349) in the coral a glycine (Gly-323) is situated at this position in Nostoc. sp. and at the position of the tyrosine in the coral (Tyr-353) a histidine (His-327) was found.

The alignment of the deduced amino acid sequence of the NspFP LOX-domain with known LOX sequences shows, that the residues involved in iron binding within the active site are highly conserved and identical to those from plant LOXs: His-170, His-175, His342 and Asn-346. Although the fifth ligand did not align properly to the other sequences, it is represented by an isoleucine (Ile-429) as for other plant LOXs (Fig. 2B, li). At the position of the three determinants that may be involved in regiospecificity, NspFP contains different amino acids in comparison to the other LOX sequences from flowering plants (Sloane et al. 1995; Borngräber et al. 1996b; Hornung et al. 1999a): At the site described first by Sloane, a serine (Ser-229) is found instead of a phenylalanine in both plant LOXs (LOX2 At2 and PpLOX1; Fig. 2B, sl). These amino acids differ remarkably from the reported determinants for positional specificity of plant LOXs (Hornung et al. 1999a), precluding accurate prediction of positional specificity of the LOX-domain. In addition, an Arg residue reported to determine inverse substrate orientation in plant LOXs is missing as well from the NspFP (Fig. 2B, ho)(Hornung et al. 1999a). However, the amino acid residue determining the stereospecificity of LOXs (Coffa site, Fig. 2B, cof) is an alanine residue, suggesting that this enzyme is a $S$-specific LOX (Coffa and Brash 2004). 
A)

Nostoc

Plexaura

1

.

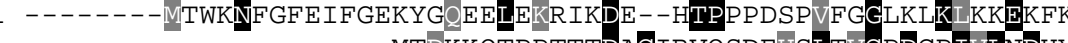

oryza 1741 GSLLLPGCVLQENVILGALSVAPENAVLRRGGVYVGSQSPAMVKNTLLDEDERIEEMDQA

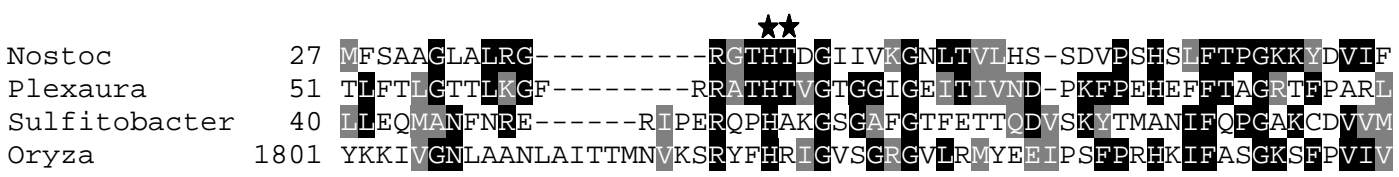

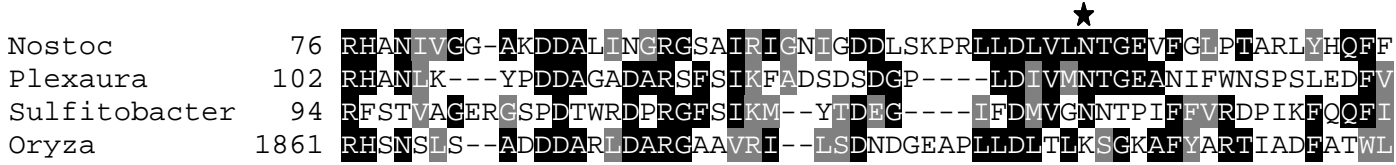

Nostoc 135 GSDFHQKSDMLASGSLRRY- -AVEAALRNPDSFTELYYHTQLCYEWVDSKKKSRYARFRL

Plexaura 155 PVEEGDAAEEYVYKNPYYYYNLVEALRRAPDTFAHLYYYSQVTMPFKAKDGKVRYCRYRA

Sulfitobacter 148 RSQKRRADNNMRDHDMQW--DFWTLSPESAHQVTYLMGDRGIPKNWREMNGYSSHTYSLV

oryza 1917 VCGLPAREEQVKRSPHIR- -DAVWGSLRSTDSYTVLHYYSNICRLLRFDDGREMYAKFKL

Nostoc 193 L- - - -NPNQS TEGGLLDDSVEIGPRLVLPRKRGDTREKNYLRNEFRORLTDGN-II-VEYV Plexaura 215 LPGDVDIKEEDESGRLTEE-EQRKIWIFSRHENEKRPDDYLRKEYVERLQKGP-VNYRLQ Sulfitobacter 206 NAEGEKFWVKF-HFHTDOGDGNAYLSQDEADKLAGTNGDYHRADLFNNIRDGNYPSWTLK oryza 1975 RPADPDVPEDS-GKVVPRGILPPETGAIPRDEDDTRPLLFLADDFRRRVGSPDGVRYVFQ

Nostoc 247 LQAQFRSIEDVA- -V--DCSNIWDPNTYPWLDIAAIVLNQDESENDYYQEIAYNPGNTHY Plexaura 273 IQI IEASPDDTA--TIFHAGILWDKETHPWFDLAKVSIKTPLSP-DVLEKTAFNIANQPA Sulfitobacter 265 WQIMPYEDAKTYRINPFDLTKVWPHEDYPLIEVGKLTLNRN--PTDFHTEIEQAAF- - - E Oryza 2034 LQLREVPTDAAARDVALDCTRPWDEAEFPYIDVGEVSIGRNL-PTEETEKLEFNPFLRCP

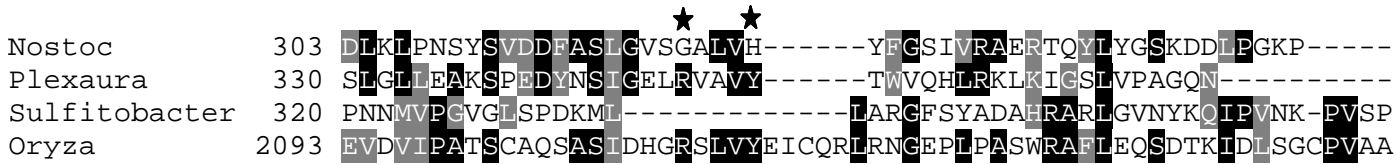


B)

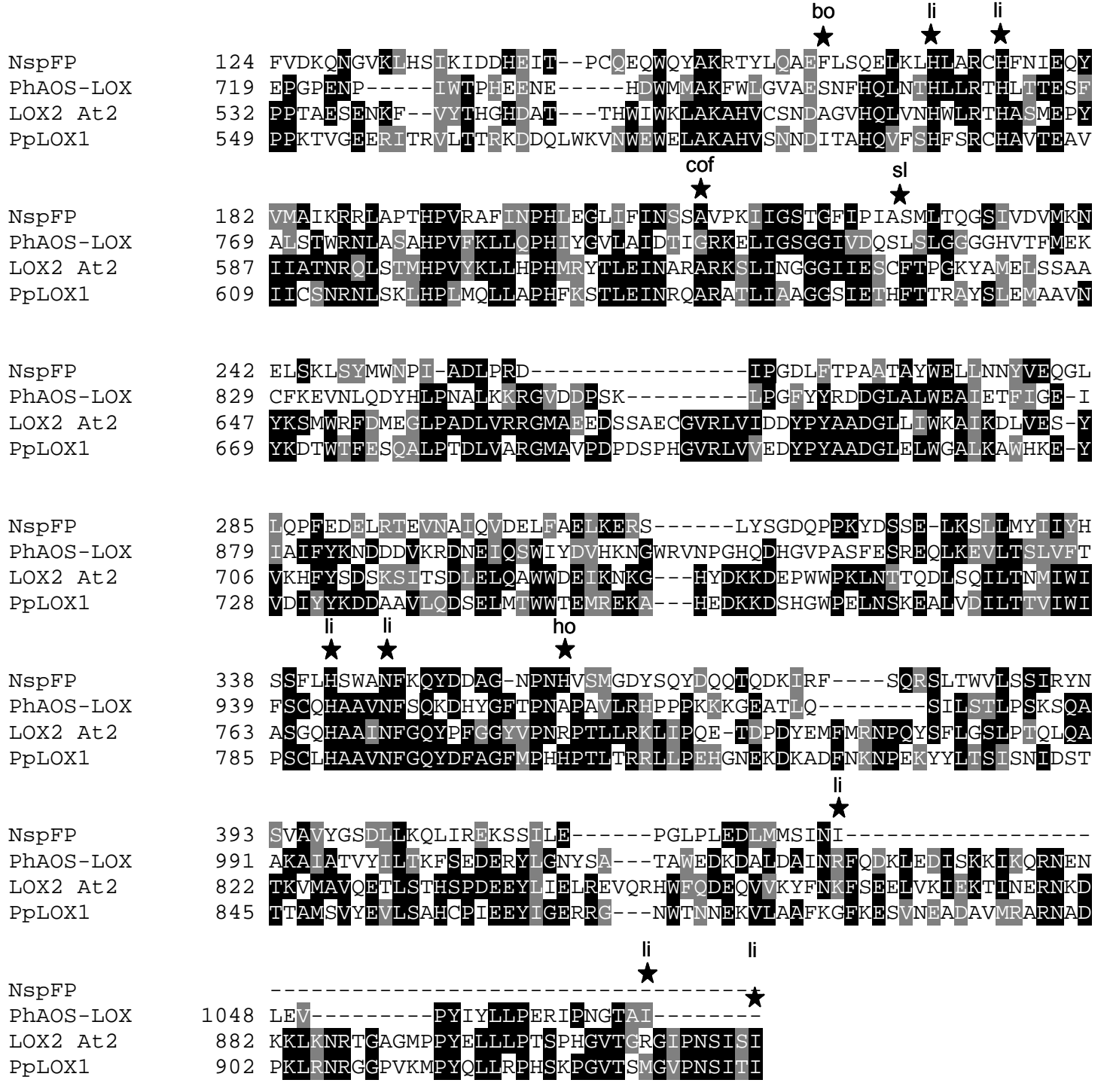

Figure 25. Partially deduced amino acid sequence alignment of peroxidase and LOX domain of $\boldsymbol{N}$. sp. SAG 25.82 fusion protein. LOX2 At2 (Acc. no. AAF79461) from $A$. thaliana, PpLOX1 (Acc. no CAE47464) from P. patens, 13-LOX (Acc. no. AAL85880) PhAOS-LOX (Acc. no. AAC47283) from the coral P. homomalla, peroxidase (Acc. no XP_472850) from O. sativa and peroxidase (Acc. no. ZP_00955421) from S. sp. EE-36.

A) stars: conserved amino acids which are involved in the active site of the AOS coding domain of the fusion protein of $P$. homomalla and which are also present in the peroxidase domain of the fusion protein $N$. sp. B) stars: the residues involved in iron ligation (three histidines, an asparagine and an isoleucine; $l i$ ), and the three determinants for substrate and regiospecificity (bo, according to Borngraber et al. 1996, sl, according to Sloane et al. 1995, ho, according to Hornung et al. 1999). 
To isolate the fusion protein and the separate domains, gene specific primers were used to amplify the full length fusion protein and the separate domains from genomic DNA of $N$. sp SAG 25.82. The obtained fragment of the full length fusion protein had a complete open reading frame of $2322 \mathrm{bp}$, encoding a protein of 774 amino acids with a molecular weight of $70.9 \mathrm{k}$. The analysis of the three obtained fragments via gel electrophoresis showed, that the peroxidase domain as well as the full length fusion protein were about $1300 \mathrm{bp}$ longer than expected from the published genomic sequence of NspFP (data not shown). Sequence analysis of the two fragments in question revealed the presence of a large transposon insertion within the catalase coding domain causing a frame shift. This insertion had highest homology to an insertion sequence, named IS891N putative transposase gene, which was identified in Nostoc ellipsosporum (Acc. no. U48694). Consequently, the insertion was removed via PCR mutagenesis to allow expression of the catalase coding domain and the intact fusion protein. To investigate the catalytic activities of the fusion protein and the separate domains, we made three different constructs, which were overexpressed in E. coli (DE3) expression strains.

\subsubsection{The LOX domain of Nostoc sp is a linoleate (9R)-LOX}

The activity of the LOX domain of Nostoc sp SAG 25.82 was tested with several free FA substrates and linoleic acid methyl esters. The enzyme was active with all free substrates and converted LA, ALA and GLA predominantly to the corresponding 9-hydroperoxides (Fig. 26). AA was mainly converted to $(5 Z, 8 Z, 11 Z, 12 E, 14 Z)-11$-hydroxy-5,8,12,14eicosatetraenoic acid (11-HETE). In contrast to the free FAs, the meLA seemed not to be a suitable substrate as there was no product formation detectable (data not shown). CPHPLC analysis revealed that the main products of the FA conversion occurred in the ' $R$ ' configuration. Therefore the LOX-domain was identified as a linoleate $(9 R)$-LOX, since the FA analysis of Nostoc sp. suggested any C20 FA as a substrates for the LOX-domain of the fusion protein 


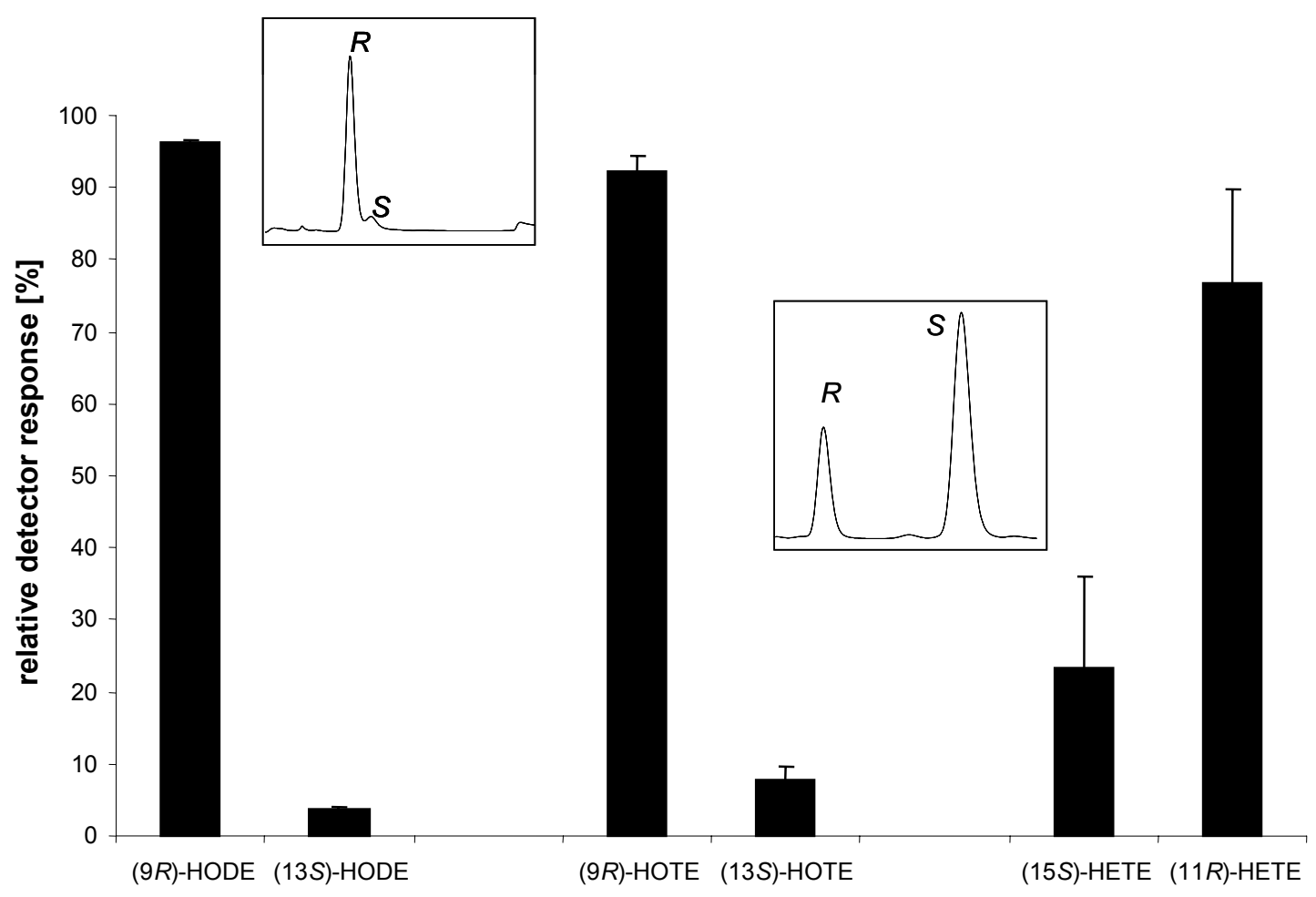

Figure 26. HPLC analysis of reaction products of the LOX domain. Amounts of the hydroxy FAs are given in relative percentage. Products of LA ((9R)-HODE and (13S)HODE), ALA ((9R)-HOTE and (13S)-HOTE) and AA ((15S)-HETE and (11R)-HETE) are shown as black bars. The left insert represents the ratio of enantiomer of 9-HODE, 9HOTE and 11-HETE analysed by CP-HPLC. The right insert represents the ratio of enantiomers of 13-HODE, 13-HOTE and 15-HETE. Each value represents an average of two independent analyses, the standard deviation is given.

The pH-optimum of the LOX domain was investigated by measuring the oxygen consumption during substrate conversion with an $\mathrm{O}_{2}$-electrode. The highest activity of the LOX domain was between $\mathrm{pH} 7$ and $\mathrm{pH}$ 10, and the activity decreased dramatically above pH 10.5 (Fig. 27). 


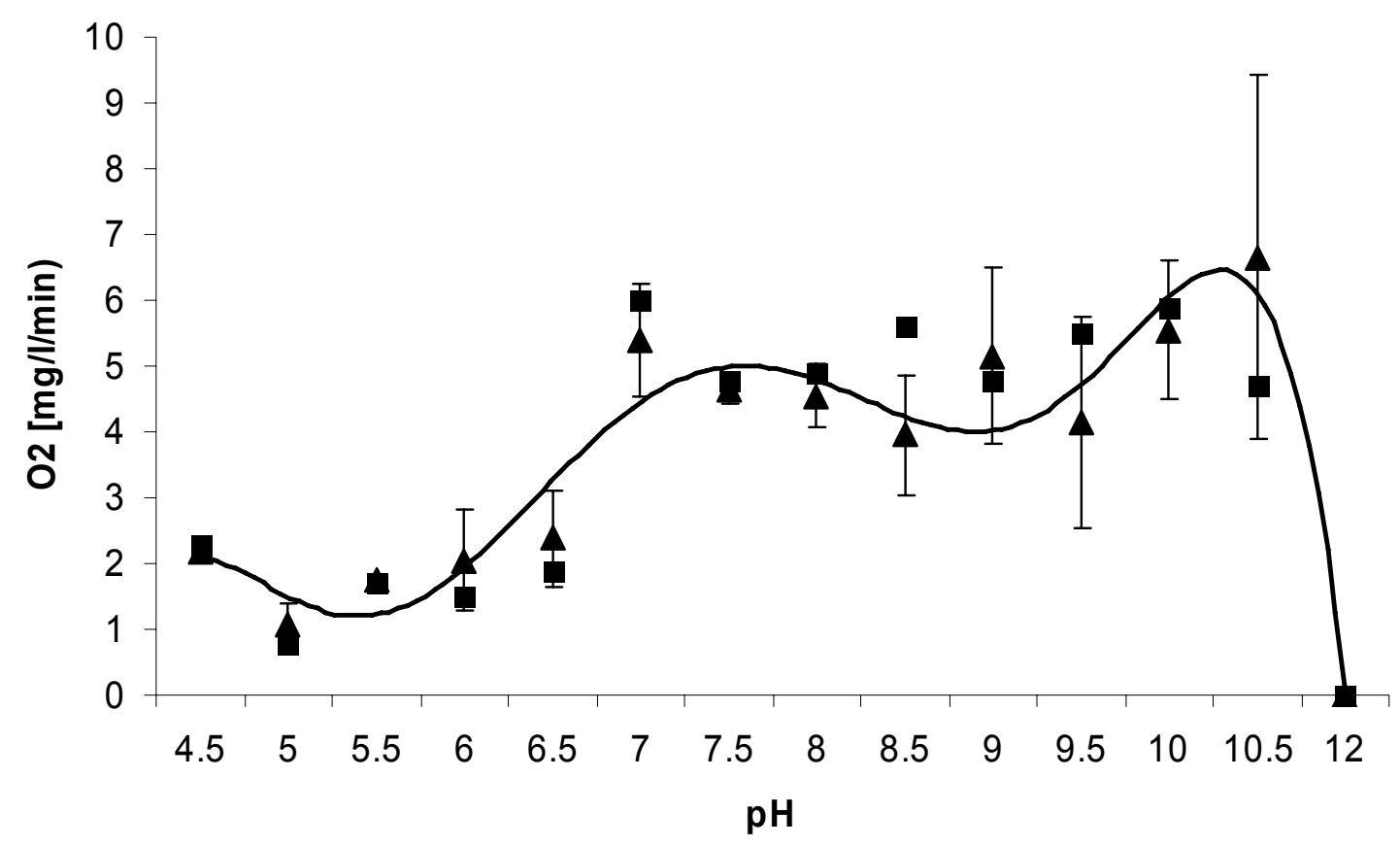

Figure 27. pH-optimum of NspFP with ALA. The pH-optimum was obtained by measuring the $\mathrm{O}_{2}$ consumption during ALA conversion with an oxygen electrode. Mean values represents an average of two independent analysis. $\boldsymbol{\Delta}=$ LOX domain, $\boldsymbol{\square}=$ NspFP.

Furthermore the preferred substrates of the LOX domain were determined by incubating the supernatant of cell lysates of E. coli cultures expressing the LOX domain with a mixture of LA, ALA, GLA and AA. The decline of the FAs by incubation with the recombinant protein in comparison to the empty-vector control was measured via GC (10.5). The data showed that ALA was the main substrate converted by the LOX-domain (70\% of the substrate was converted), whereas LA GLA and AA were converted to a lesser extent (LA $42 \%$ GLA $54 \%$ and AA $46 \%$ ) (Fig. 28 ). 


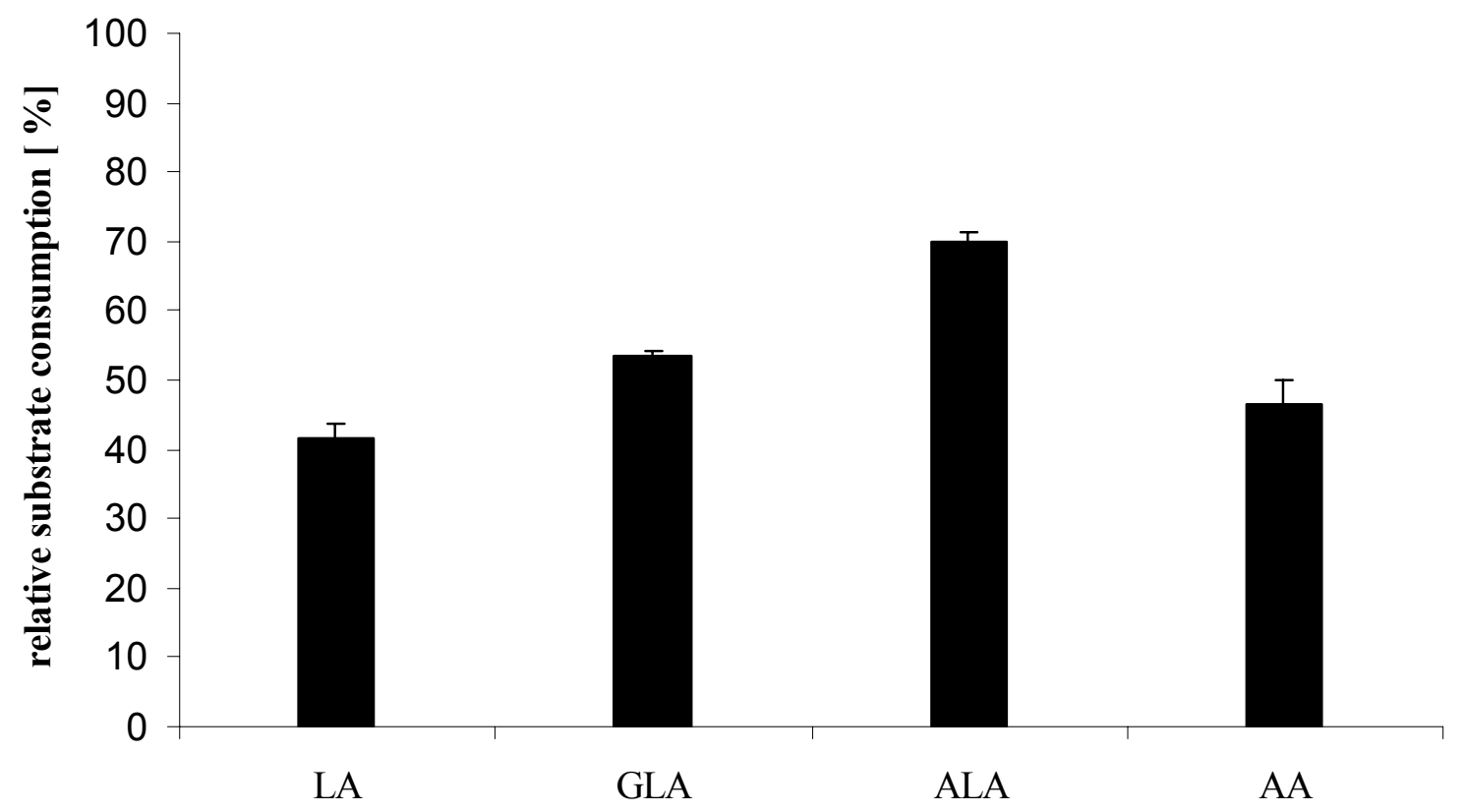

Figure 28. Substrate preference of LOX domain. The LOX domain was incubated with a mixture of LA, ALA, GLA and AA. The decline of the FAs by incubating with the recombinant protein in comparison to the empty-vector control was measured via GC. Mean values represent three independent experiments.

\subsubsection{The peroxidase domain is enzymatically not active with the reaction products of}

\section{the LOX domain}

To characterise the activity of the peroxidase coding domain the supernatant of the cell lysate was incubated with the products of the LOX-reaction: (9R)-HPODE and (13S)-HPODE. In addition the corresponding hydroxides (9R)-HODE and (13S)-HODE were tested. The products were analysed via HPLC. With all tested substrates, including $\left[1-{ }^{14} \mathrm{C}\right]-(9 S)-$ HPODE, no activity was detectable. Western blot analysis with an antibody against the amino terminal His-tag confirmed an expression of the enzyme (Fig. 29). Therefore it was concluded, that the peroxidase-domain was not active, possibly because of unfavourable in vitro conditions or misfolding of the protein domain. 


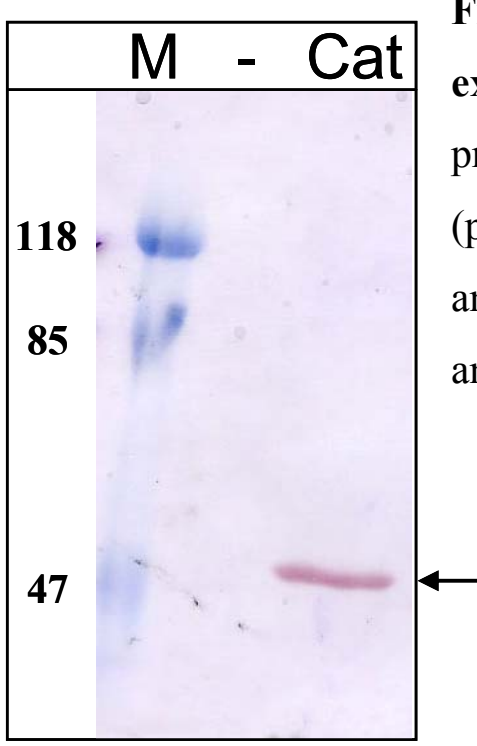

Figure 29. Western blot analysis of the peroxidase domain expressed in $E$. coli BL21 expression cells. M, prestained protein marker (Fermentas, St. Leon Rot), - , empty vector (pET15b) control, Cat, peroxidase domain. Primary antiserum: mouse anti tetra-His, secondary antiserum: goat anti mouse-AP conjugated.

\subsubsection{Production of dihydroxy fatty acids by the NspFP}

Next, NspFP was tested by incubating the supernatant of the cell lysate of E. coli cells containing expressed NspFP with free FAs (LA, ALA, GLA and AA) as substrates. The products were analysed with RP-HPLC, HPLC/MS, GC/MS and NMR. In the HPLC analyses one main peak and several smaller peaks were observed in the chromatogram (Fig. 30). All products were more polar than the monohydroperoxide resulting from the LOX reaction and had absorption maxima at $232 \mathrm{~nm}$. HPLC/MS-analysis identified these products as dihydroxide isomers. Furthermore GC/MS analysis of silylated derivatives of the products yielded information about the position of the two hydroxide groups. In the corresponding mass spectra the mass fragments generated confirmed the proposed structure of 9,14-diHODE (Fig. 31A, Tab. 16). The mass spectrum was characterised by ions at $m / z 439\left[\mathrm{M}-31 \text {, loss of } \mathrm{OCH}_{3}\right]^{+}, m / z 413$ [M-57, loss of $\left.\mathrm{CH}_{3}-\left(\mathrm{CH}_{2}\right)_{3}\right]^{+}, m / z 380$ [M90; loss of $\left.\mathrm{Me}_{3} \mathrm{SiOH}\right]^{+}, m / z 323$ [M-147; loss of $\mathrm{CH}_{3}-\left(\mathrm{CH}_{2}\right)_{3}$ and $\left.\mathrm{Me}_{3} \mathrm{SiOH}\right]^{+}, m / z 259$ $\left[\mathrm{Me}_{3} \mathrm{SiO}-\mathrm{CH}-\left(\mathrm{CH}_{2}\right)_{7} \mathrm{COO}-\mathrm{CH}_{3}\right]^{+}, \quad m / z 223 \quad\left[\mathrm{CH}_{3}-\left(\mathrm{CH}_{2}\right)_{2}-\mathrm{CH}=\mathrm{CH}-\mathrm{CH}=\mathrm{CH}-\mathrm{CH}=\mathrm{CH}-\mathrm{CH}-\right.$ $\left.\mathrm{OMe}_{3} \mathrm{SiO}\right]^{+}, \quad m / z \quad 211 \quad\left[\mathrm{CH}_{3}-\left(\mathrm{CH}_{2}\right)_{3}-\mathrm{CH}-\left(\mathrm{OMe}{ }_{3} \mathrm{Si}\right)-\mathrm{CH}=\mathrm{CH}-\mathrm{CH}=\mathrm{CH}\right]^{+}, \quad m / z \quad 191$ [intramolecular fragment: $\left.\left(\mathrm{OMe}_{3} \mathrm{Si}\right)_{2}-\mathrm{CH}\right]^{+} ; m / z 159\left[\mathrm{CH}_{3}-\left(\mathrm{CH}_{2}\right)_{3}-\mathrm{CH}-\left(\mathrm{OMe}_{3} \mathrm{Si}\right)\right]^{+}$and $m / z$ $73\left[\mathrm{Me}_{3} \mathrm{Si}^{+}\right]$.

The E,E double bond configuration of 9,14-diHODE was detected via NMR-analysis (Tab. 15). The ${ }^{1} \mathrm{H}$ and ${ }^{13} \mathrm{C}$ chemical mass shifts were in agreement with those of $(7 E, 9 E)$ hexadeca-7,9-diene-6,11-diol, but distinct from those of (7Z,9Z)-hexadeca-7,9-diene-6,11diol (Tolstikov et al. 1989). 
The main product of the LA-conversion was identified as $(10 E, 12 E)-9,14$-dihydroxy10,12-octadecadienoic acid (9,14-diHODE). Two other substances were identified as $(11 E, 13 E)-9,10$-dihydroxy-11,13-octadecadienoic acid (9,10-diHODE) and (9E,12E)-8,11dihydroxy-9,12-octadecandienoic acid (8,11-diHODE). The corresponding ion fragments $(\mathrm{m} / \mathrm{z}$ and relative intensities) are given in Tab. 16. Several additional side products were not identified so far.

For the conversion of ALA, GLA and AA an equivalent HPLC pattern was obtained with one main product and several side products. The main products were $(10 E, 12 E, 14 E)-9,16-$ dihydroxy-10,12,14-octadecatrienoic acid (9,16-diHOTE) for ALA, $(6 Z, 10 E, 12 E)$-9,14dihydroxy-6,10,12-octadecatrienoic acid (9,14-diHOTE) for GLA and (5Z,8Z,12E,14E)11,16-dihydroxy-5,8,12,14-eicosatetraenoic acid (11,16-diHETE) for AA. The most prominent ions in the mass spectra of 9,16-diHOTE were as follows in $\mathrm{m} / \mathrm{z}$ and ion structure: 437 [M-31, loss of $\left.\mathrm{OCH}_{3}\right]^{+}, 378$ [M-90; loss of $\left.\mathrm{Me}_{3} \mathrm{SiOH}\right]^{+}, 337$ [M-131, loss of $\mathrm{CH}_{3}-\mathrm{CH}_{2}-\mathrm{CH}-\left(\mathrm{OMe} \mathrm{Si}_{3}\right]^{+}, \quad 259 \quad\left[\mathrm{Me}_{3} \mathrm{SiO}-\mathrm{CH}-\left(\mathrm{CH}_{2}\right)_{7} \mathrm{COO}-\mathrm{CH}_{3}\right]^{+}, 209 \quad\left[\mathrm{CH}_{3}-\mathrm{CH}_{2}-\mathrm{CH}-\right.$ $\left.\left(\mathrm{OMe}_{3} \mathrm{Si}\right)-\mathrm{CH}=\mathrm{CH}-\mathrm{CH}=\mathrm{CH}-\mathrm{CH}=\mathrm{CH}\right]^{+}, 191$ [intramolecular fragment: $\left.\left(\mathrm{OMe}_{3} \mathrm{Si}\right)_{2} \mathrm{CH}\right]^{+}$, $131\left[\mathrm{CH}_{3}-\mathrm{CH}_{2}-\mathrm{CH}-\left(\mathrm{OMe}_{3} \mathrm{Si}\right)\right]^{+}$. For 9,14-diHOTE the following ions in $\mathrm{m} / \mathrm{z}$ and ion structure were found: 313 [M-155, loss of $\left.\mathrm{CH}_{2}-\mathrm{CH}=\mathrm{CH}-\left(\mathrm{CH}_{2}\right)_{4}-\mathrm{COOCH}_{3}\right]^{+}, 256\left[\mathrm{Me}_{3} \mathrm{SiO}-\right.$ $\left.\mathrm{CH}-\mathrm{CH}=\mathrm{CH}-\mathrm{CH}=\mathrm{CH}-\mathrm{CH}-\mathrm{OMe}_{3} \mathrm{Si}\right]^{+}, \quad 223 \quad\left[\mathrm{CH}_{3}-\left(\mathrm{CH}_{2}\right)_{2}-\mathrm{CH}=\mathrm{CH}-\mathrm{CH}=\mathrm{CH}-\mathrm{CH}=\mathrm{CH}-\mathrm{CH}-\right.$ $\left.\mathrm{OMe}_{3} \mathrm{SiO}\right]^{+}, 191$ [intramolecular fragment: $\left.\left(\mathrm{OMe}_{3} \mathrm{Si}\right)_{2}-\mathrm{CH}\right]^{+}$and $159\left[\mathrm{CH}_{3}-\left(\mathrm{CH}_{2}\right)_{3}-\mathrm{CH}-\right.$ $\left.\left(\mathrm{OMe}_{3} \mathrm{Si}\right)\right]^{+}$. The most prominent ions in the mass spectra of 11,16-diHETE were as follows in $\mathrm{m} / \mathrm{z}$ and ion structure: $313\left[\mathrm{Me}_{3} \mathrm{SiO}-\mathrm{CH}-\mathrm{CH}=\mathrm{CH}-\mathrm{CH}=\mathrm{CH}-\mathrm{CH}-\left(\mathrm{OMe}_{3} \mathrm{Si}\right)-\right.$ $\left.\left(\mathrm{CH}_{2}\right)_{3}-\mathrm{CH}_{3}\right]^{+}, \quad 283 \quad\left[\mathrm{Me}_{3} \mathrm{SiO}-\mathrm{CH}-\mathrm{CH}_{2}-\mathrm{CH}=\mathrm{CH}-\mathrm{CH}_{2}-\mathrm{CH}=\mathrm{CH}-\left(\mathrm{CH}_{2}\right)_{3}-\mathrm{COOCH}_{3}\right]^{+}, \quad 256$ $\left[\mathrm{Me}_{3} \mathrm{SiO}-\mathrm{CH}-\mathrm{CH}=\mathrm{CH}-\mathrm{CH}=\mathrm{CH}-\mathrm{CH}-\mathrm{OMe}_{3} \mathrm{Si}\right]^{+}, 211 \quad\left[\mathrm{CH}_{3}-\left(\mathrm{CH}_{2}\right)_{3}-\mathrm{CH}-\left(\mathrm{OMe}_{3} \mathrm{Si}\right)-\mathrm{CH}=\mathrm{CH}-\right.$ $\mathrm{CH}=\mathrm{CH}]^{+}, \quad 191$ [intramolecular fragment: $\left.\left(\mathrm{OMe}_{3} \mathrm{Si}\right)_{2}-\mathrm{CH}\right]^{+}, \quad 159 \quad\left[\mathrm{CH}_{3}-\left(\mathrm{CH}_{2}\right)_{3}-\mathrm{CH}-\right.$ $\left.\left(\mathrm{OMe}_{3} \mathrm{Si}\right)\right]^{+}$.

So far it was not possible to identify all the side products of the reaction.

The pH-optimum of NspFP was determined with an $\mathrm{O}_{2}$-electrode. In general the $\mathrm{pH}$ optimum was similar to that of the LOX domain alone. Highest activity was observed in the $\mathrm{pH}$ range between 7 and 10.5 (Fig. 27). 


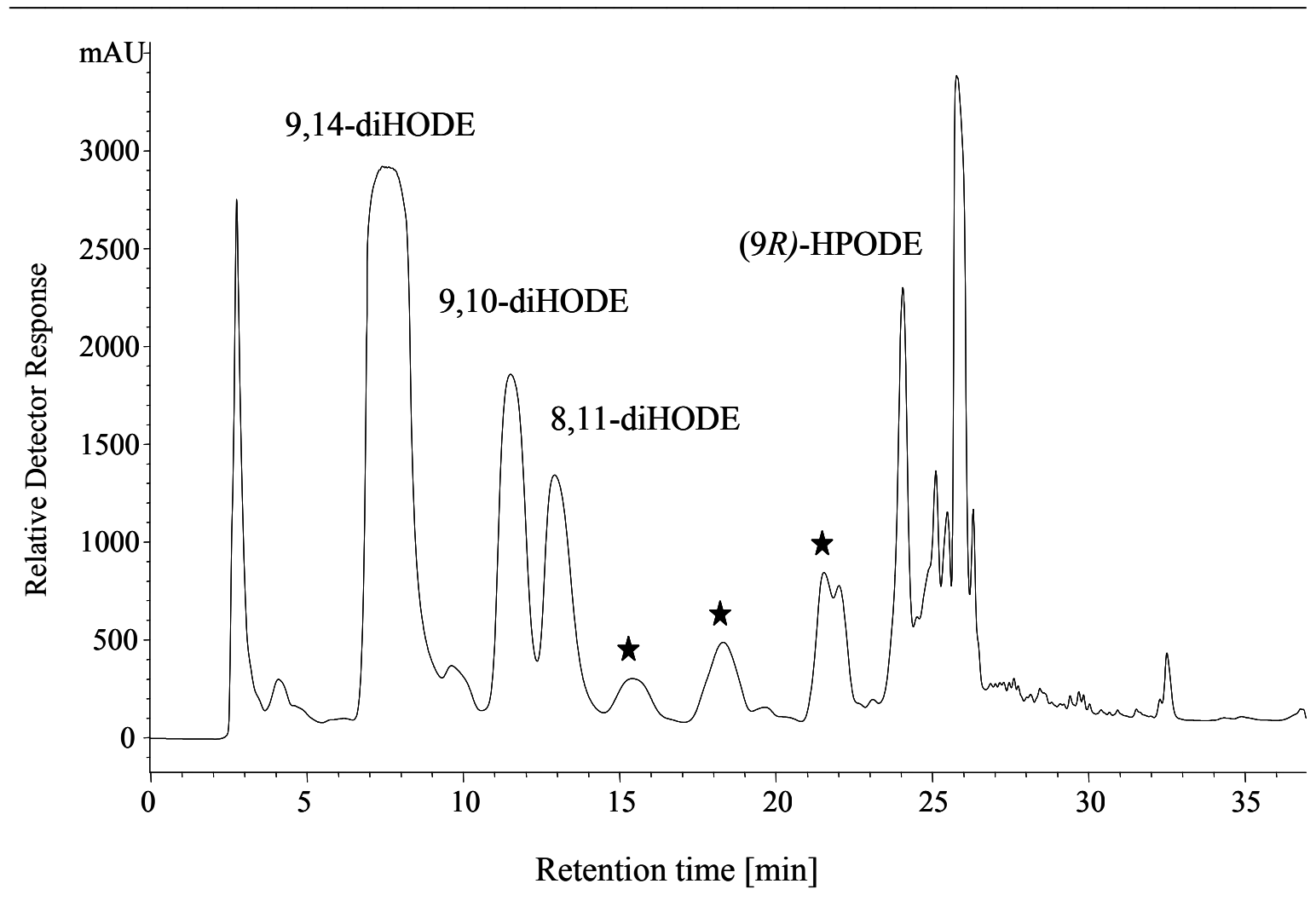

Figure 30. Separation of the products of LA conversion catalysed by the NspFP using RP-HPLC. The reaction was carried out in $50 \mathrm{mM}$ Tris/HCl, $\mathrm{pH}$. The main products 9,14-diHODE, 9,10-diHODE and 8,11-diHODE as well as the intermediate (9R)-HODE are marked, peaks marked with a star represent products not reliably identified so far. Unmarked peaks are endogenous substances of E. coli expression strain BL21. The chromatogram represents one out of at least 15 experiments.

Table 15. Selected NMR data of 9,14-diHODE (solvent $\mathrm{CDCl}_{3}$ )

\begin{tabular}{lll}
\hline $\mathrm{H}$ & $\delta{ }^{1} \mathrm{H}[\mathrm{ppm}]$ & $\delta^{13} \mathrm{C}[\mathrm{ppm}]^{\mathrm{b}}$ \\
\hline 8,15 & $1.54^{\mathrm{a}}$ & n.d. \\
9,14 & 4.15 & 72.7 \\
10,13 & 5.70 & 136.4 \\
11,12 & 6.20 & 129.8 \\
\hline
\end{tabular}

a $\quad{ }^{1} \mathrm{H}$ chemical shifts of ${ }^{1} \mathrm{H},{ }^{1} \mathrm{H}$ COSY $2 \mathrm{D}$ NMR correlation peaks

b $\quad{ }^{13} \mathrm{C}$-chemical shifts of ${ }^{1} \mathrm{H},{ }^{13} \mathrm{C}$ HSQC 2D NMR correlation peaks

n.d. not detected because of poor signal/noise ratio

NMR-analysis of 9,14-diHODE 
Table 16. Overview of the dihydroxy FAs formed in the reaction of NspFP with different FA substrates. The analysis of products was done with HPLC, LC/MS and GC/MS. For each substance the absorption maximum [nm] and fragment sizes [m/z] are given.

\begin{tabular}{|c|c|c|c|c|c|}
\hline LA & $\begin{array}{l}\text { HPLC } \\
\text { LC/MS } \\
\text { GC/MS }\end{array}$ & $\begin{array}{l}\mathrm{nm} \\
m / z \quad \text { (fragment } \\
\left.\text { ions, }[\mathbf{M}-\mathbf{H}]^{-}\right) \\
m / z \\
\text { (rel. intensity) }\end{array}$ & 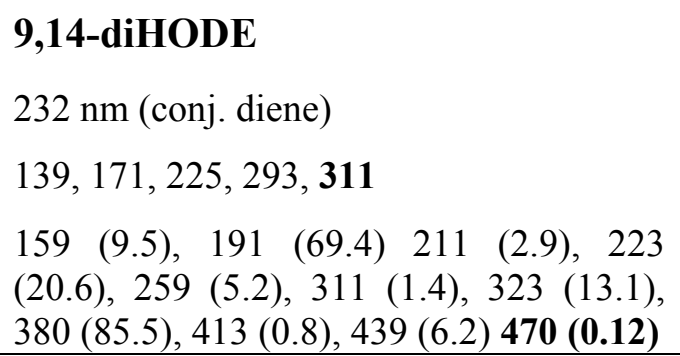 & $\begin{array}{l}\text { 9,10-diHODE } \\
232 \mathrm{~nm} \text { (conj. diene) } \\
139,171,201,293, \mathbf{3 1 1} \\
109(9.3), 155(18.6), 211(17.7), \\
259(100), 360(0.1), \mathbf{4 7 0}(\mathbf{0 . 0 1})\end{array}$ & $\begin{array}{l}\text { 8,11-diHODE } \\
232 \mathrm{~nm} \text { (conj. diene) } \\
\text { No MS/MS } \\
129(34.9), 143 \quad(3.3), 155 \\
(9.3), 199(100), 285(0.27), \\
327(0.36), \mathbf{4 7 0}(\mathbf{0 . 0 3})\end{array}$ \\
\hline ALA & $\begin{array}{l}\text { HPLC } \\
\text { LC/MS } \\
\text { GC/MS }\end{array}$ & $\begin{array}{l}\mathrm{nm} \\
m / z \quad \text { (fragment } \\
\left.\text { ions, }[\mathbf{M}-\mathbf{H}]^{-}\right) \\
\mathrm{m} / \mathrm{z} \\
\text { (rel. intensity) }\end{array}$ & $\begin{array}{l}\text { 9,16-diHOTE } \\
232 \mathrm{~nm} \text { (conj. triene: } 260 / 268 / 281) \\
137,171,251, \mathbf{3 0 9} \\
\begin{array}{llll}131 \quad(38.7), 191 & (5.9) 209 & (3.5), 259 \\
(11.6), 311(3.9), 337(8.7), \mathbf{4 6 8}(\mathbf{1 . 4})\end{array}\end{array}$ & $\begin{array}{l}\text { 9,13-diHOTE } \\
232 \mathrm{~nm} \text { (conj. diene) } \\
137,171,239, \mathbf{3 0 9} \\
243(6.5), 259(100), 299(4.3), \\
401(0.07), \mathbf{4 6 8}(\mathbf{0 . 0 4})\end{array}$ & \\
\hline GLA & $\begin{array}{l}\text { HPLC } \\
\text { LC/MS } \\
\text { GC/MS }\end{array}$ & $\begin{array}{l}\mathrm{nm} \\
m / z \quad \text { (fragment } \\
\left.\text { ions, }[\mathbf{M}-\mathbf{H}]^{-}\right) \\
\mathrm{m} / \mathrm{z} \\
\text { (rel. intensity) }\end{array}$ & $\begin{array}{l}\text { 9,14-diHOTE } \\
232 \mathrm{~nm} \text { (conj. diene) } \\
137,171,251, \mathbf{3 0 9} \\
159(6.8), 191 \quad(100), 223 \quad(11.5), 256 \\
(1.2), 313(17.1), \mathbf{4 6 8}(\mathbf{0 . 0 1})\end{array}$ & & \\
\hline $\mathbf{A A}$ & $\begin{array}{l}\text { HPLC } \\
\text { LC/MS } \\
\text { GC/MS }\end{array}$ & $\begin{array}{l}\mathrm{nm} \\
m / z \quad \text { (fragment } \\
\text { ions, }\left[\mathbf{M}-\mathbf{H}^{-}\right]^{-} \\
\mathrm{m} / \mathrm{z} \\
\text { (rel. intensity) }\end{array}$ & $\begin{array}{l}\text { 11,16-diHETE } \\
232 \mathrm{~nm} \text { (conj. diene) } \\
167,249,335 \\
159(5.1), 191(100), 211(0.7), 256(2.1) \\
283(0.18), 313(14.5), \mathbf{4 9 4}(\mathbf{0 . 0 2})\end{array}$ & & \\
\hline
\end{tabular}




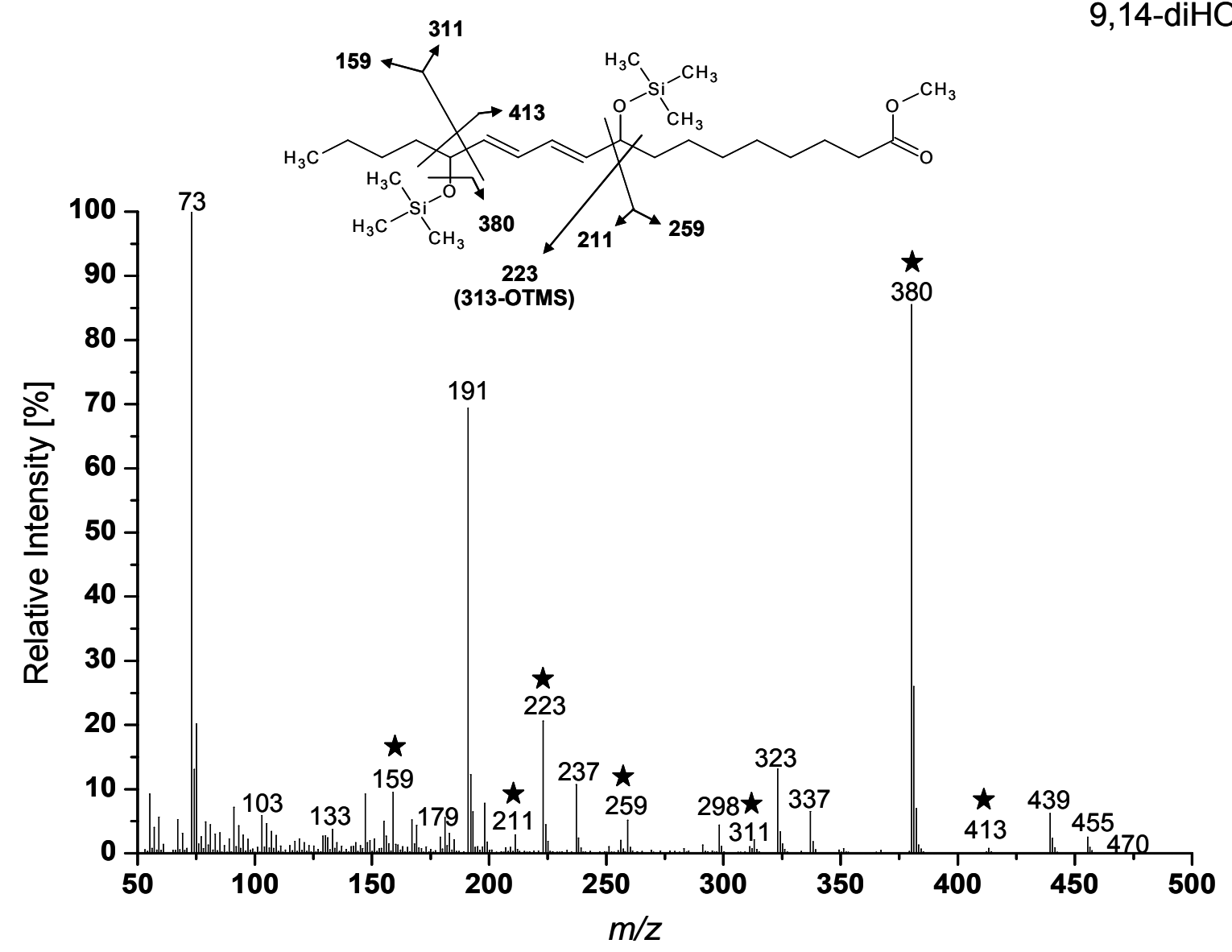

B

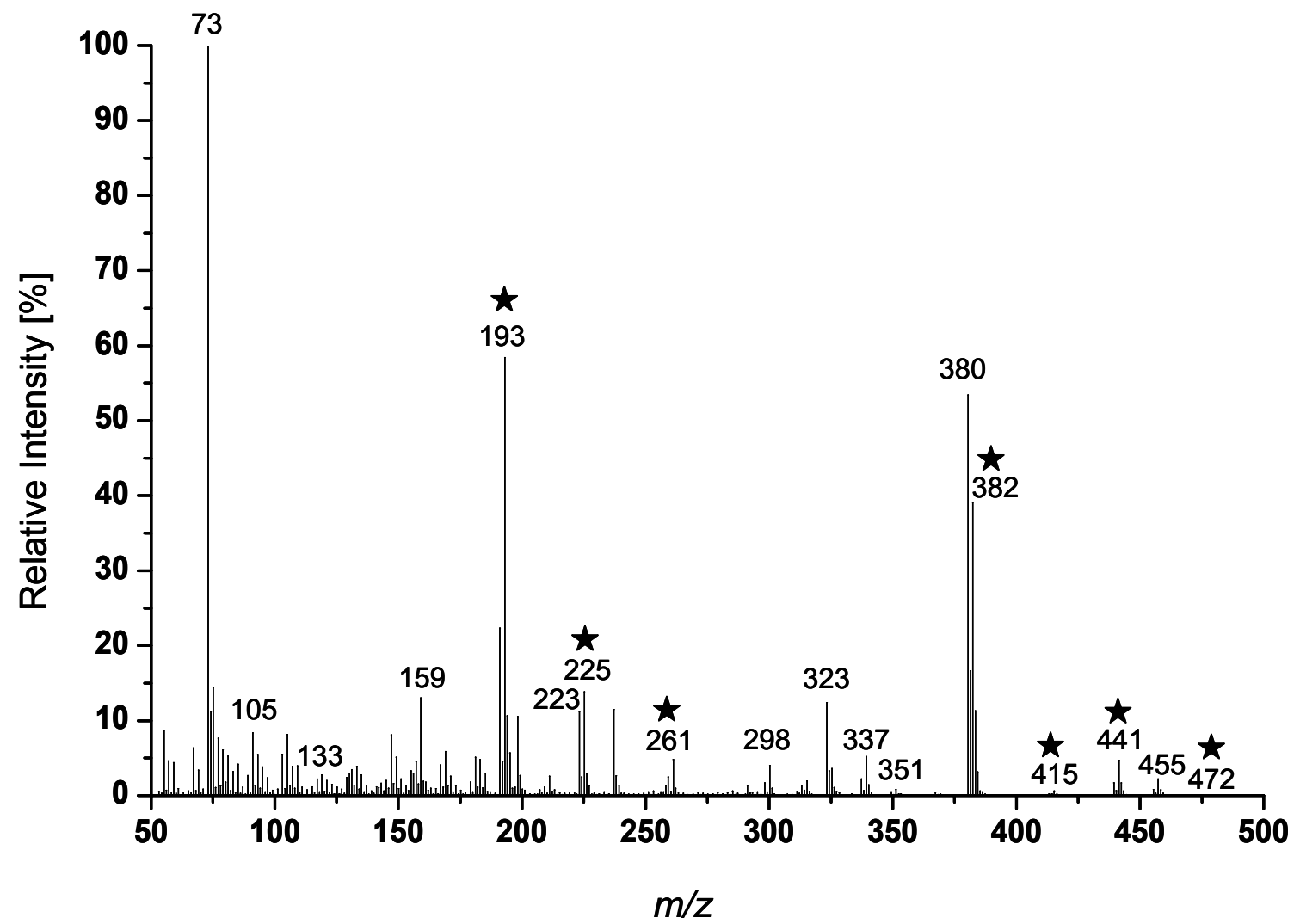


C

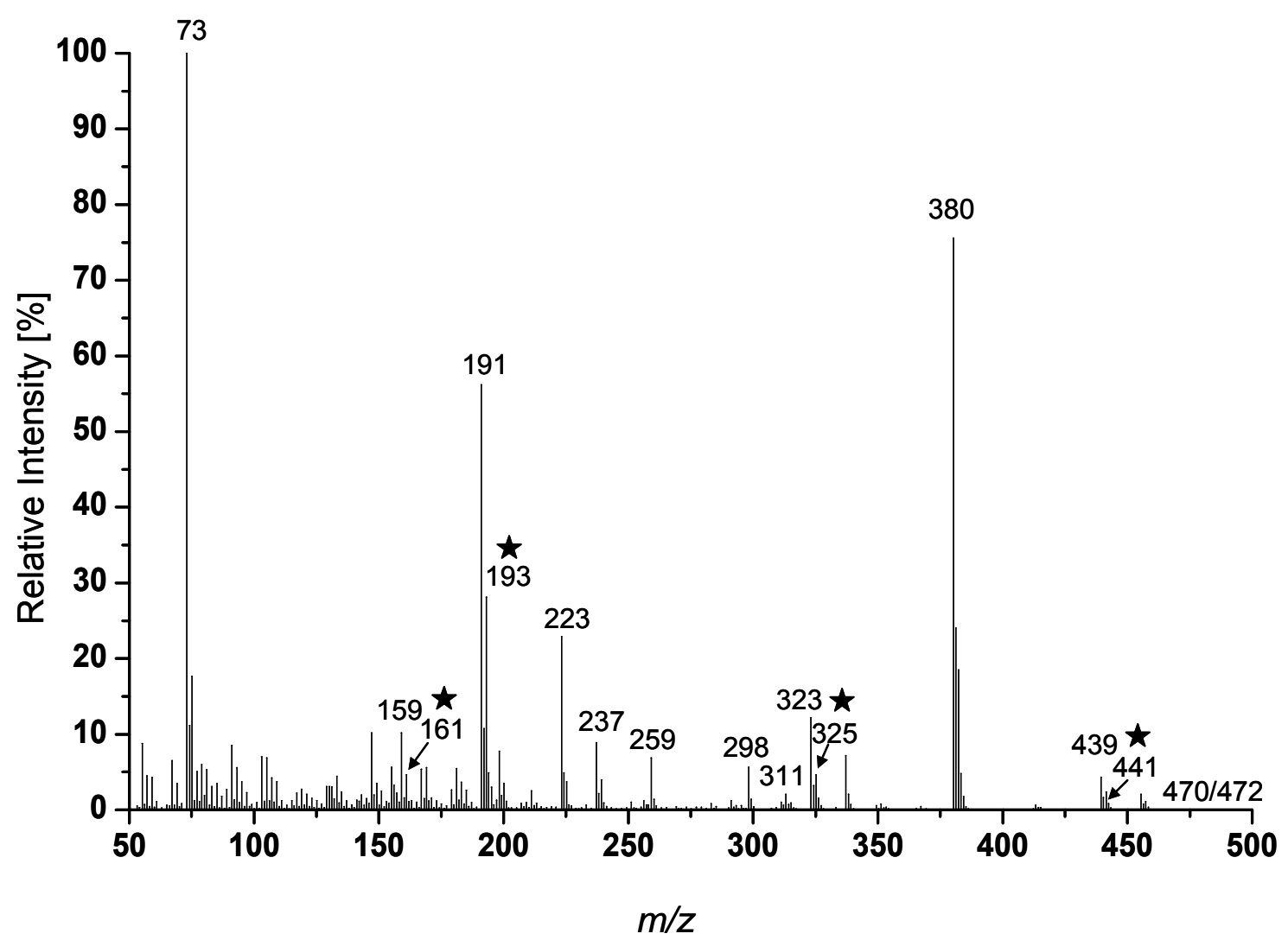

Figure 31. GC/MS analysis of 9,14-diHODE, converted by NspFP. A) Mass spectrum of 9,14-diHODE, Stars indicate the mass fragments which were deduced from the proposed structure B) Mass spectrum of 9,14-diHODE derived from the conversion of LA in presence of ${ }^{18} \mathrm{O}_{2}$. Stars indicate the mass fragments which were deduced from a mass shift resulting from the incorporation of $\left.{ }^{18} \mathrm{O}, \mathbf{C}\right)$ mass spectrum of 9,14-diHODE derived from the conversion of LA in presence of $\mathrm{H}_{2}{ }^{18} \mathrm{O}$. Stars indicate the mass fragments which were deduced from a mass shift resulting from the incorporation of ${ }^{18} \mathrm{O} . \mathrm{m} / \mathrm{z} 73$ and $\mathrm{m} / \mathrm{z}$ 191 derive from derivatisation reagent TMS. The experiment was repeated once with identical results.

\section{Kinetic analysis of LA conversion by NspFP}

The AOS domain of the coral fusion protein converts the hydroperoxide to an unstable allene oxide, which further hydrolyses to ketols nonenzymatically. To exclude the formation of unstable intermediates during the NspFP reaction kinetic analyses were performed (9.7). The conversion of LA by NspFP was stopped at different time points and the products were analysed via RP-HPLC. A detection of any unstable epoxide or other similar intermediates was not possible. The synthesis of the dihydroxy FAs started 
immediately after adding LA to the reaction mix (Fig. 32). The data showed, that (9R)-HPODE as well as the dihydroxides were already detectable after $20 \mathrm{~s}$, with 9,14-diHODE being the major product. The amount of 9,14-diHODE increased within the first $15 \mathrm{~min}$ by threefold, then a slight decrease within the next $15 \mathrm{~min}$ was detectable. From 30 to 90 min the amount of 9,14-diHODE did not change, but the last $30 \mathrm{~min}$ of the experiment (from 90 to $120 \mathrm{~min}$ ) a twofold increase was observed again. The curve progression of the two other dihydroxides was similar to that of 9,14-diHODE, but the amount was significantly lower (about tenfold). Furthermore the intermediate (9R)-HPODE was constantly present. Anyway, a slight decrease of (9R)-HPODE between 1 to $15 \mathrm{~min}$ was observed, whereas the amount of dihydroxides was increasing as described before. These results suggest that the dihydroxides were direct products of the peroxidase activity.

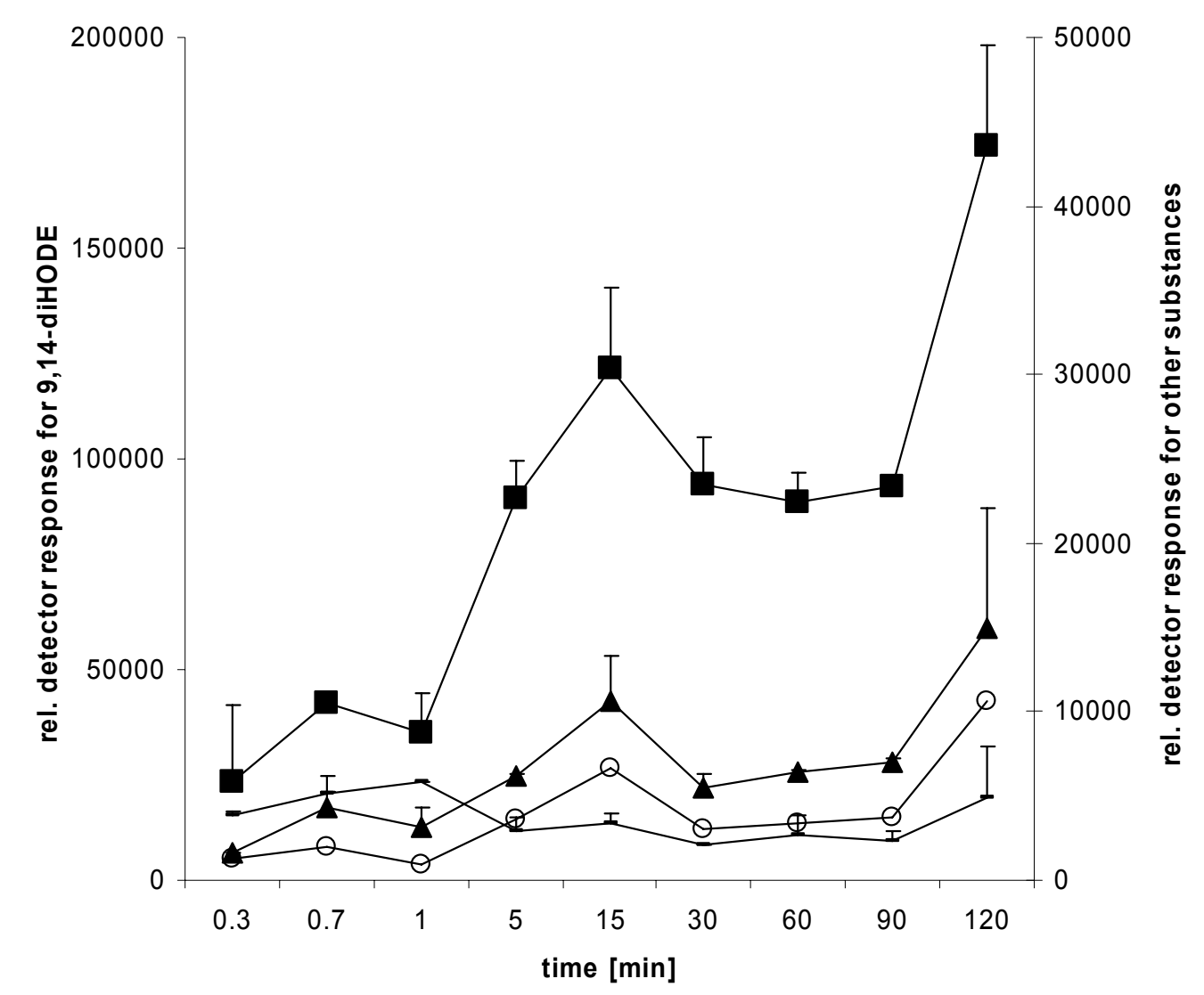

Figure 32. Kinetic analysis of LA conversion catalysed by NspFP. The reaction was carried out in $50 \mathrm{mM}$ Tris/ $\mathrm{HCl} \mathrm{pH} 8$ and stopped at different time points. The products were analyzed by RP-HPLC and the main products ((9R)-HPODE, 9,14-diHODE, 9,10diHODE and 8,11-diHODE) are shown in the graphic. Each value represents an average of two independent analyses, standard deviation is given. $\boldsymbol{\square}=9,14$-diHODE, $\boldsymbol{\Delta}=9,10$ diHODE, o = 8,11-diHODE, $-=9$-HPODE 


\section{Reaction mechanism of NspFP}

To understand the reaction mechanism of NspFP the origin of the second oxygen was investigated. As evident from the first oxygen which derived from molecular oxygen and which is substrate for the LOX. The second oxygen could either derive from the peroxide group of $(9 R)$-HPODE or from water. To test this, we performed an activity test in presence of either ${ }^{18} \mathrm{O}_{2}$ or $\mathrm{H}_{2}{ }^{18} \mathrm{O}$. Similarly, the products were analysed by GC/MS and HPLC/MS. The mass spectra of the experiment with ${ }^{18} \mathrm{O}_{2}$ revealed that only the first oxygen atom at $\mathrm{C} 9$ derived from ${ }^{18} \mathrm{O}_{2}$, whereas the second oxygen atom remained unlabelled (Fig. 31B). The fragments having the C9 atom with the first hydroxy group and deriving from the LOX reaction, contained the labelled oxygen atom $(\mathrm{m} / \mathrm{z} 472, \mathrm{~m} / \mathrm{z} 441$ $\left[\mathrm{M}-31 \text {, loss of } \mathrm{OCH}_{3}\right]^{+}, m / z 415\left[\mathrm{M}-57 \text {, loss of } \mathrm{CH}_{3}-\left(\mathrm{CH}_{2}\right)_{3}\right]^{+}, m / z 261\left(\mathrm{Me}_{3} \mathrm{Si}\left[{ }^{18} \mathrm{O}\right]-\mathrm{CH}-\right.$ $\left.\left(\mathrm{CH}_{2}\right)_{7} \mathrm{COO}-\mathrm{CH}_{3}\right]^{+}$and $\mathrm{m} / z \quad 225 \quad\left[\mathrm{CH}_{3}-\left(\mathrm{CH}_{2}\right)_{2}-\mathrm{CH}=\mathrm{CH}-\mathrm{CH}=\mathrm{CH}-\mathrm{CH}=\mathrm{CH}-\mathrm{CH}-\right.$ $\left[{ }^{18} \mathrm{O}\right] \mathrm{Me}_{3} \mathrm{Si}^{+}$. Contrary, the mass fragments obtained containing the second hydroxy group at $\mathrm{C} 14$ were labelled, when LA was converted in the presence of $\mathrm{H}_{2}{ }^{18} \mathrm{O}$ (Fig 31C). The mass spectra showed mass ions $m / z$ 441(M-31, loss of $\left.\mathrm{OCH}_{3}\right), m / z 325$ [M-147; loss of $\mathrm{Me}_{3} \mathrm{SiOH}$ and $\left.\mathrm{CH}_{3}-\left(\mathrm{CH}_{2}\right)_{3}\right]^{+}$and $m / z 161\left(\mathrm{CH}_{3}-\left(\mathrm{CH}_{2}\right)_{3}-\mathrm{CH}-\left(\left[{ }^{18} \mathrm{O}\right] \mathrm{Me}_{3} \mathrm{Si}\right)\right]^{+}$.

The data indicate that the fusion protein catalysed the conversion of the FA substrate in a two step reaction: First the LOX domain converts LA to $(9 R)$-HPODE, which is then further metabolised to $(10 E, 12 E)-9,14-$ diHODE by the peroxidase domain. The oxygen atom of the hydroxy group at position 14 does not derive from the peroxy group of $(9 R)$ HPODE but from water.

\subsubsection{Comparison of endogenous oxylipins in different cyanobacterial strains}

So far, the in vitro characterisation of NspFP isolated from the $N$. sp. strain SAG 25.82, which is a duplicate strain to $N$. sp PCC7120 as kept in the Pasteur Culture Collection, (PCC, Paris, France) was described. Analysis of the isolated sequences showed, that SAG 25.82 and PCC7120 both harbour an insertion of a transposon in the NspFP sequence, as described above. In order to compare the dependency of cyanobacterial oxylipin formation on the NspFP protein, two additional N. sp. strains, PCC7120 A and P, were obtained (a gift from Prof. Peter Wolk, Michigan State University, East Lansing, MI, USA and Prof. James Golden, Texas A\&M University, College Station, TX, USA, respectively), which do not harbour a disruptive insertion in the NspFP-gene. These two strains were ordered from the PCC in Paris years ago, but cannot be regarded as specific 
mutants in the NspFP gene since these strains may slowly drift genetically as well and thus may harbour mutations in different genes.

The insertion likely results in the formation of an inactive enzyme with a deduced amino acid sequence and this may influence the oxylipin metabolism of the cyanobacteria. The identification of these two different alleles of the Nostoc gene encoding NspFP, raised interest in comparing the "wildtype" (wt) strains PCC 7120-A and -P with the "mutant" (mt) strain SAG 25.82 (8.5). In addition, it was aimed to compare endogenous products of the LOX pathway of wounded and non-wounded cyanobacteria as it is known from the literature that the LOX pathway is induced upon wounding in higher plants (Schilmiller and Howe 2005a). Therefore cyanobacterial cultures were additionally subjected to wounding by ultrasonification (10.11), and the effects on oxylipin metabolism were monitored, as described previously (Lang and Feussner 2007). In all samples free and esterified hydro(pero)xy dienoic FAs were measured.

In both non-wounded and wounded Nostoc sp. PCC7120 strains only C18-PUFA-derived hydroxides were detected, and no C16 or C20 derived oxylipins were present. In addition, traces of FA hydroperoxides were detected. The analysis of free oxylipins in the wt strains showed, that the most abundant hydroxy FAs were derived from 9-LOX activity (0.3$1.0 \mathrm{nmol} / \mathrm{g}$ f.w, Fig. 33A and B). The amount of the hydroxy FAs increased upon wounding, in particular that of 9-hydroxy FAs, which showed an almost tenfold increase with wounding (9.1-11.7 nmol/g f.w., Fig. 33A and B). The amount of the other hydroxy FAs (13-HODE, 13HOTE, 12-HOTE and 16-HOTE) increased only slightly to about $0.8 \mathrm{nmol} / \mathrm{g}$ f.w.. In comparison, the $\mathrm{mt}$ strain contained similar amounts of hydroxy FAs of about $0.7 \mathrm{nmol} / \mathrm{g}$ f.w., and there was no preference for either one of the hydroxy FA isomers (Fig. 33C). In addition, only a weak induction of oxylipin formation was observed upon wounding. The difference between wt and $\mathrm{mt}$ strains was even more remarkable when the stereochemistry of the different hydroxy FAs was analysed (Fig. 33D). The oxylipins 9-HODE and 9-HOTE of the wt strains were in $R$-configuration, whereas 9HODE and 9-HOTE of the mt strain were racemic. These data indicated an induction of $(9 R)$-LOX activity in the wt strains upon wounding and a loss of LOX activity in the mt strain, most likely due to the insertion of a disruptive transposase insert into the NspFPgene. Interestingly, neither in wt nor in $\mathrm{mt}$ strains any dihydroxylated FAs products of NspFP were detected, contrasting to the results from the in vitro tests with the recombinant enzyme. 
A

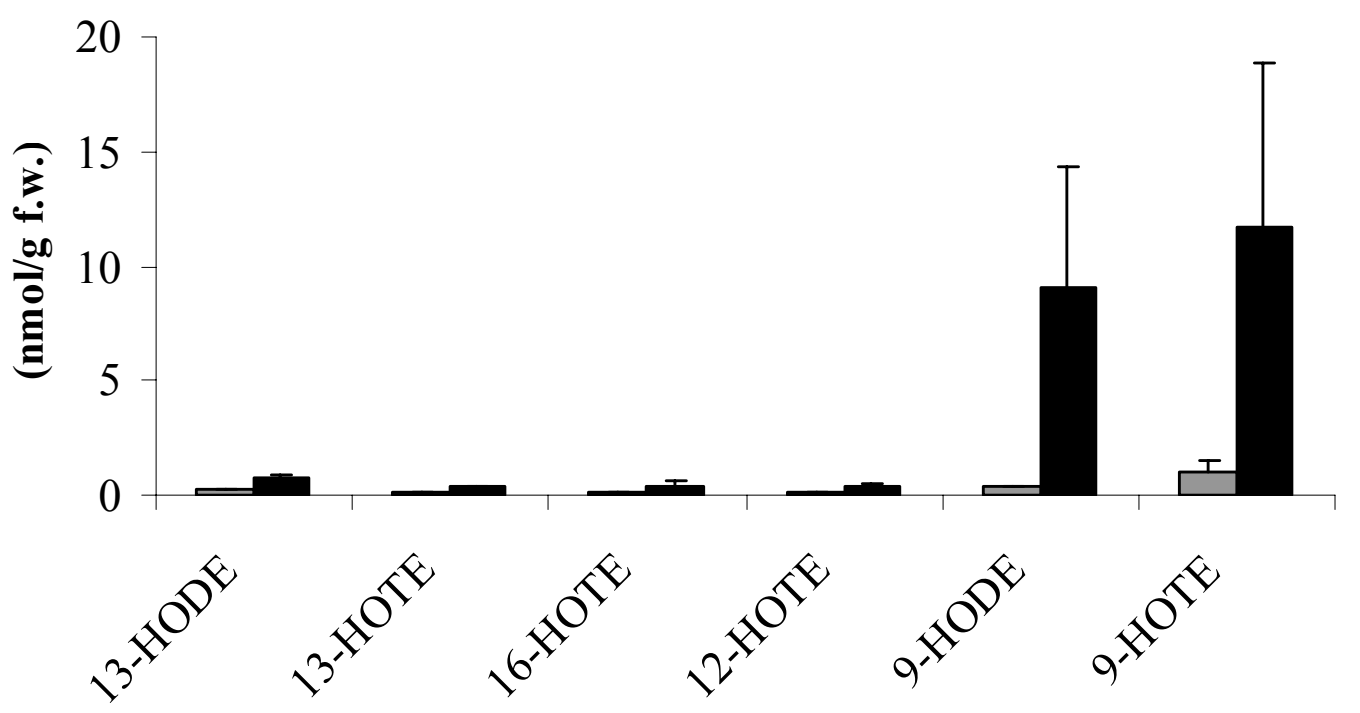

B

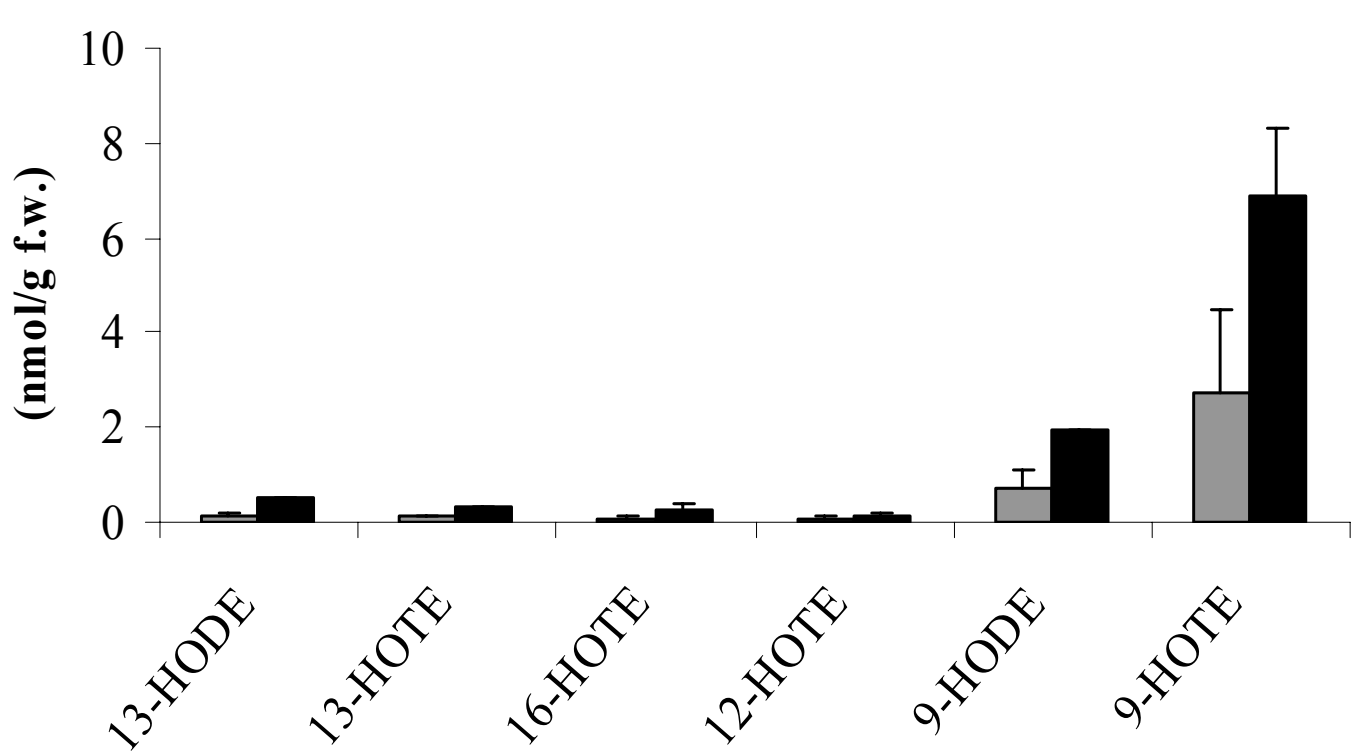


C

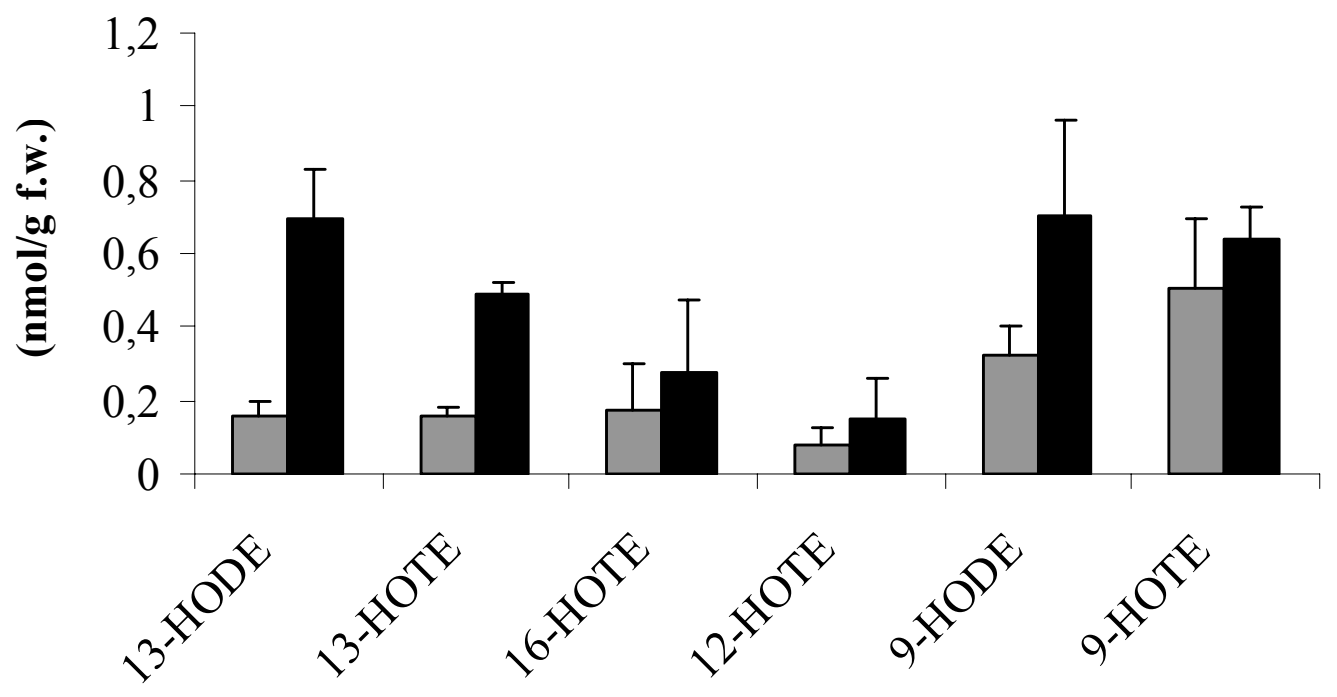

D

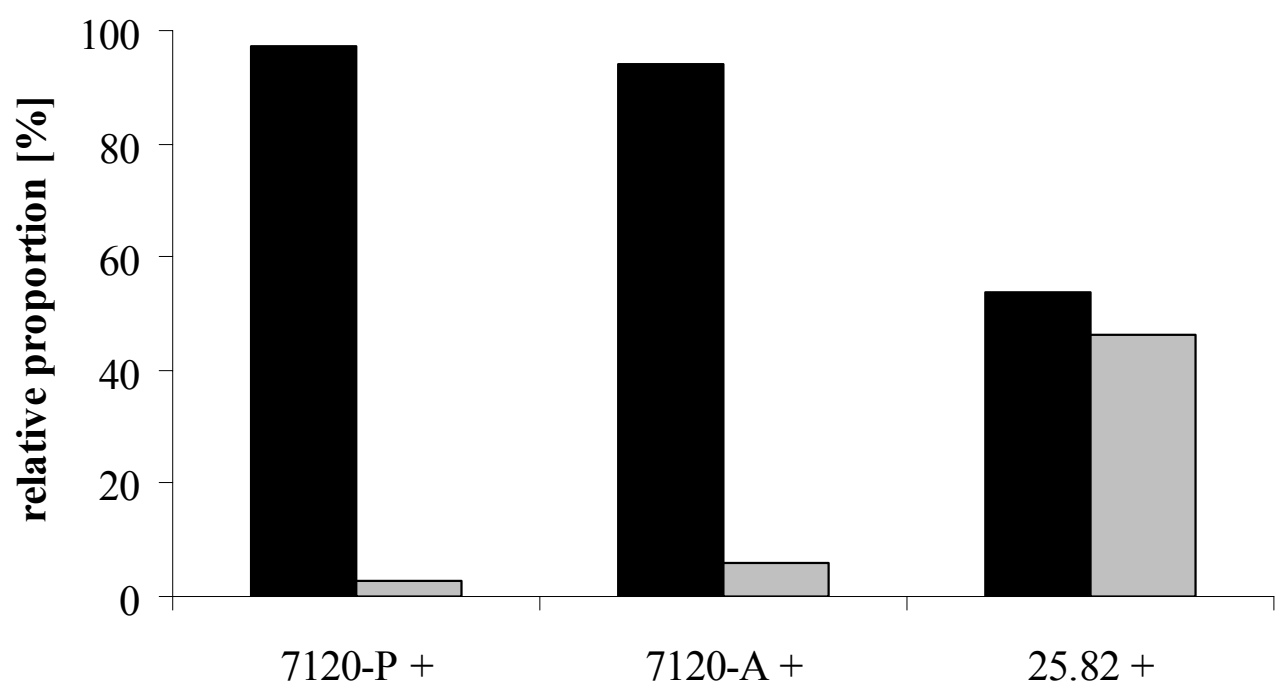

Figure 33 Free oxylipin profiles of different cyanobacterial strains. The oxylipin levels were determined in control cells as well as in wounded cells. A, Nostoc. sp. PCC7120P B, PCC7120A and C, SAG 25.82. The bars represent the amounts of detected hydroxy FAs from non-wounded (grey) or wounded (black) cyanobacteria $\mathrm{D}$, relative proportion of the $R$-(black bars) and $S$-(grey bars) enantiomers. The data represent mean values of three independent measurements. Error bars represent standard deviation. 
A

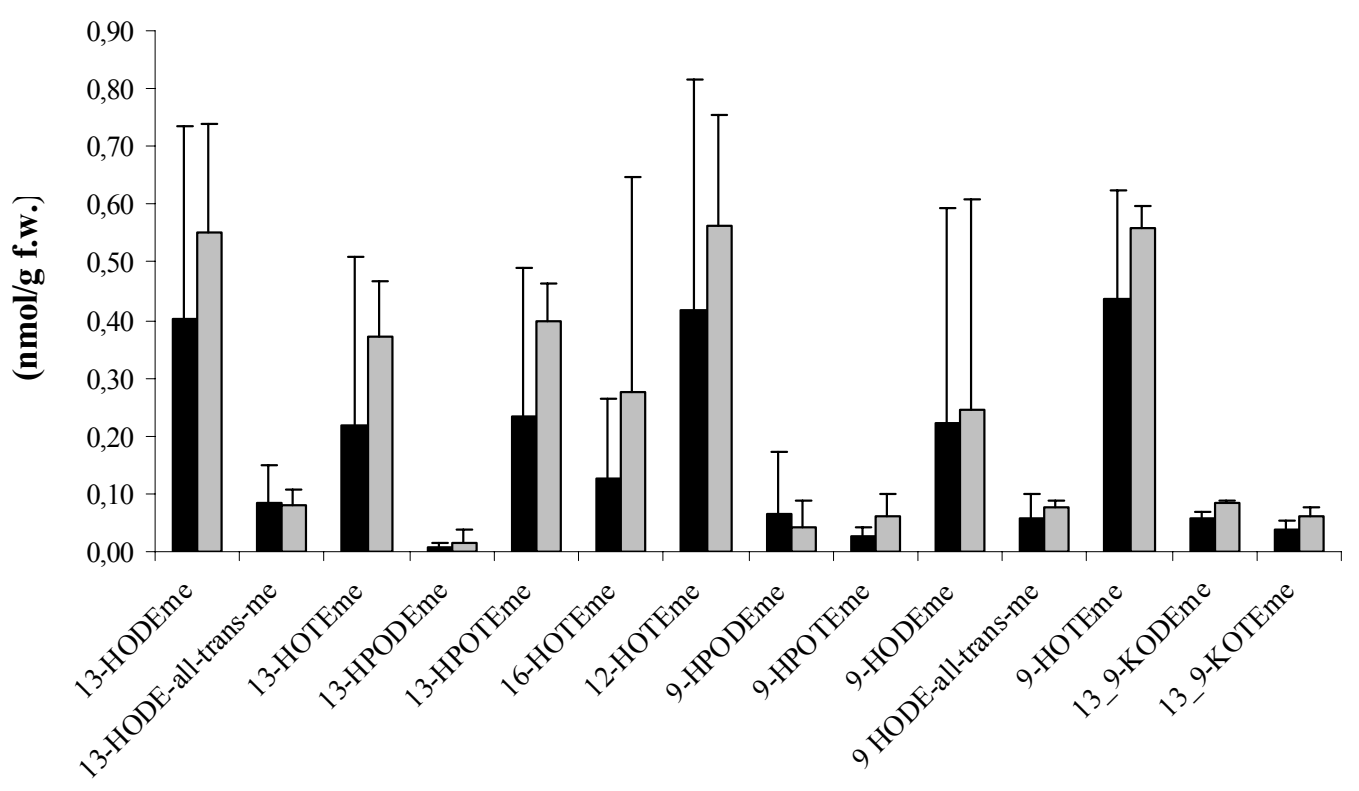

B

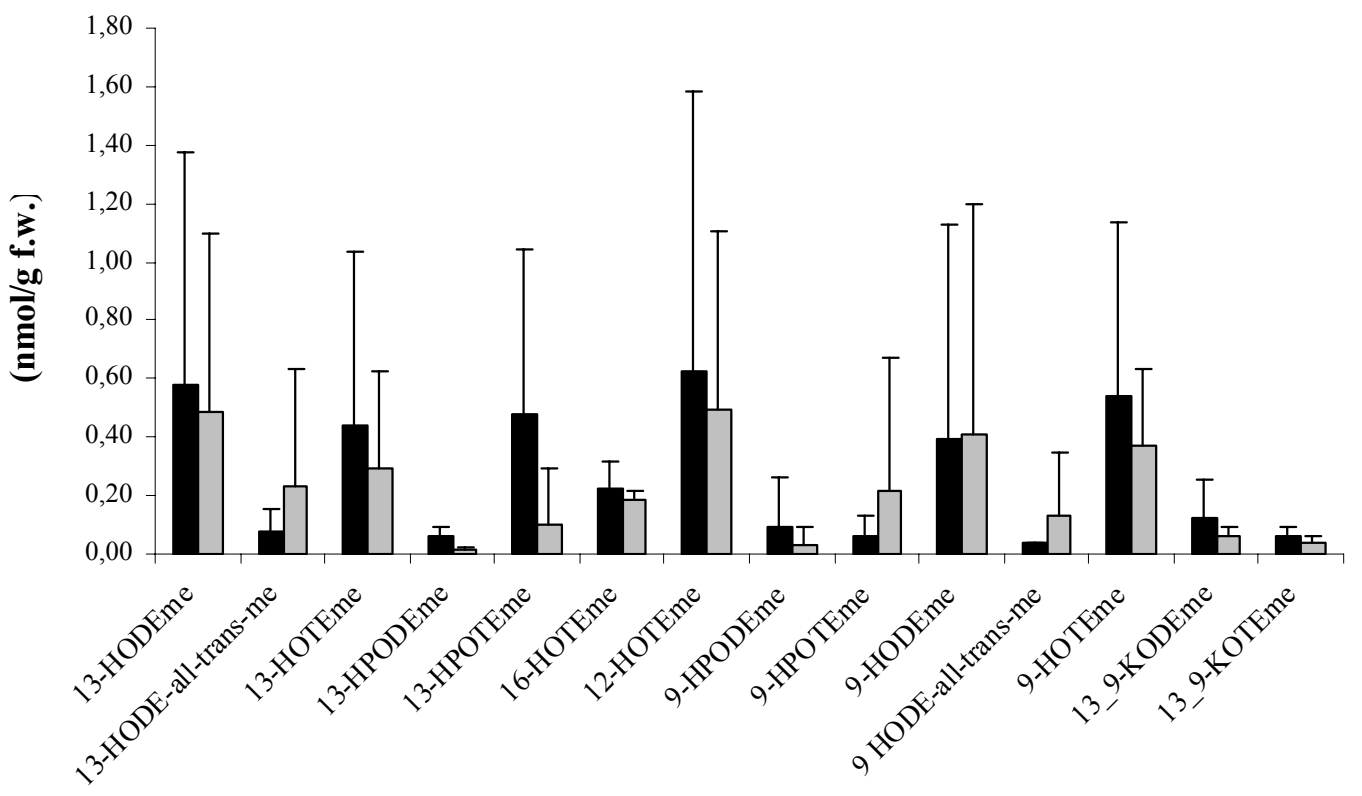




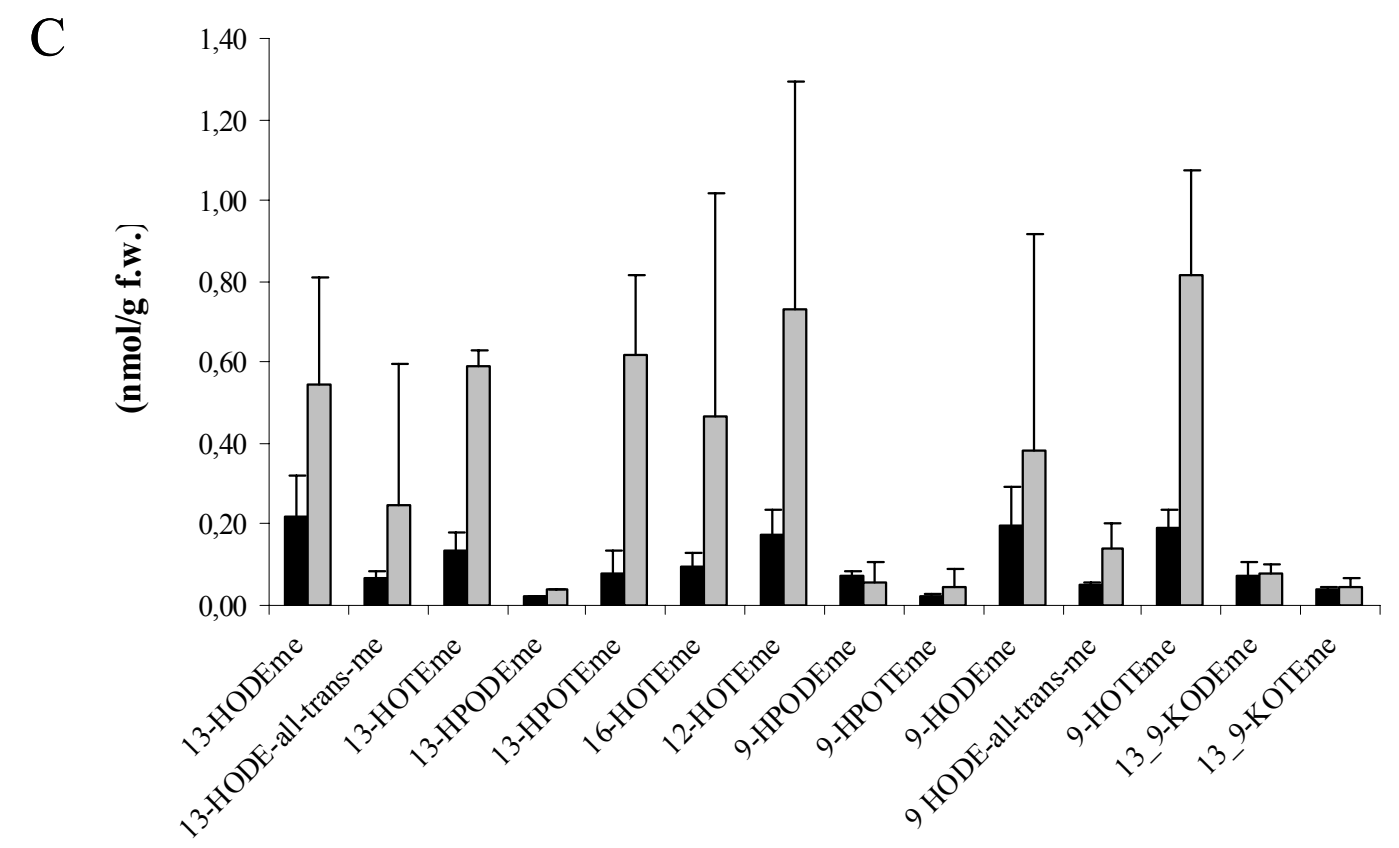

Figure 34. Esterified oxylipin profiles of different cyanobacterial strains. The oxylipin levels were determined in control cells as well as in wounded cells. The bars represent the amounts of detected hydroxy FAs from non-wounded (grey) or wounded (black) cyanobacteria. A, N. sp. PCC7120P; B, PCC7120A and C, SAG 25.82. The data represent mean values of three independent measurements. Error bars indicate standard deviations.

The analysis of oxylipins in the fraction of esterified FAs revealed that the two wt strains showed significant differences in the levels of esterified oxylipins and only for PCC7120A a slight increase of oxylipins upon wounding was detectable (Fig. 34A and B). Furthermore there was no preference for a certain hydroxy FA product like (9R)-HODE and $(9 R)$-HOTE, indicating that changes in oxylipins upon wounding are restricted to the free oxylipin fraction (Fig. 33A and B). The amount of esterified oxylipins in wt and $\mathrm{mt}$ strains showed no remarkable differences. In the case of mt strain SAG 25.85 the oxylipin level was higher in non-wounded than in wounded samples (Fig. 34C). Moreover the amount of esterified oxylipins varied between three independent measurements, resulting in high standard deviations, which are too high for any conclusions.

$\mathrm{CP}$-analysis yielded in 9-hydroxy FAs in $R$-configuration for the wt strains, which is an indication for LOX activity, whereas the 9-hydroxy FAs of the mt strain were racemic and therefore products of autoxidation. 
Results

Since the detection of any dihydroxy FAs that may derive from the activity of NspFP failed, the extractable enzyme activity that may derive from NspFP was determined. Therefore cell extracts obtained from wounded and non-wounded mt and wt strains were analysed either after incubation with LA or $\left[1-{ }^{14} \mathrm{C}\right]$-labelled LA. The samples were extracted and analyzed for products by reversed phase HPLC (RP-HPLC). In the untreated samples, formation of neither monohydroxylated nor dihydroxylated FAs was detected. Comparison of non-wounded and wounded samples however, revealed wound-induced formation of $(9 R)-\mathrm{H}(\mathrm{P}) \mathrm{ODE}$ in wt strains. Again, neither formation of 9,14-diHODE nor that of any other dihydroxy FA was detected. The results for the two wt strains were similar. For the mutant strain SAG 25.82 no oxylipins were found. 


\section{Discussion}

The genome sequencing of marine organisms and the techniques of functional genomics (including transcriptome, proteome and metabolom analysis) open up new possibilities for combining the techniques with the screening of new metabolites of biotechnological interest (Schweder et al. 2005). Moreover, the development of new analytic techniques, like coupling of chromatographic separations and mass spectrometry, allows a high through-put analysis of a broad range of metabolites, including lipids and FAs. The interest in lipids and FAs from microalgae has been stimulated by the finding that PUFAs are important for human health and nutrition (Bergé and Barnathan 2005). In particular, the omega-3 PUFAs EPA and DHA are important components of mammalian retinal and brain membranes and function in the cognitive development of infants as well as the mental health of adults. AA and EPA are also precursors of eicosanoids, including prostaglandins, leukotrienes and thrombaxanes, which are important signal molecules (Yaqoob 2003; Smith 2005).

In order to find suitable microalgal strains as a source for PUFAs and genes encoding for enzymes, which synthesise or metabolise PUFAs, the FA composition of all available SAG microalgal strains was determined in their stationary phase. The data obtained were collected into a database in Microsoft Excel format. This database provides information about composition and proportions of FAs of the SAG strains analysed and can be used to find strains producing a certain FA in high amounts or to examine their usefulness in chemotaxonomy. So far only the most eminent results of the database have been looked at and the entire information content still has to be extracted. In the following section the application of the database established is demonstrated by discussing putative microalgal strains suitable for PUFA production and plant engineering or the use of certain FAs as chemotaxonomical marker.

\section{Microalgae as sources of PUFAs and genes for plant engineering}

As mentioned before, metabolic screening has become a valuable tool in order to find a huge variety of natural compounds used in nutritional and/or pharmaceutical applications. In the present work FA profiles of microalgal strains maintained in the culture collection SAG have been obtained and evaluated. One important objective was to identify species with high contents of VLCPUFAs, in particular EPA and DHA. Furthermore it was aimed 
to identify new or unusual FAs, which are useful for chemical industry or human nutrition. Related to this the identification of enzymes involved in their biosynthesis was also followed.

\section{Microalgal strains suitable for commercial production of PUFAs}

Although some cyanobacteria, like Nostoc, Spirulina and Aphanizomenon species have been used for food supplementation for thousands of years (Jensen et al. 2001), the cultivation of microalgae in large photo bioreactors is only a few decades old (Borowitzka 1999). Commercial large-scale cultures started in the 1960's in Japan with the microalga Chlorella, followed by the industrial cultivation of the green algae Dunaliella and Haematococcus as sources for $\beta$-carotene and astaxanthin (Spolaore et al. 2006).

Generally, microalgae used in human nutrition are marketed in different forms, like tablets, capsules and liquids, whereas microalgae used in animal nutrition are often used fresh as sole component or as food additive. However, microalgae have to fulfil various criteria, like easy cultivation and nontoxicity (Brown et al. 1999; Renaud et al. 2002). Several species have demonstrated a potential for the industrial production of VLCPUFAs like EPA, but until now only algal oil rich in DHA derived from Crypthecodinium and Schizochytrium is commercially available (Spolaore et al. 2006). The main problem of using microalgae as sources for PUFAs is still that microalgal oil is not economically competitive with other sources (Apt and Behrens 1999; Chini Zittelli et al. 1999).

Nevertheless, some SAG microalgal strains with high levels of certain VLCPUFAs can be tested for their industrial production potential. Since for most strains from the class Dinophyceae large scale cultivation is technically demanding, because they are very sensitive to changes of environment, they can be excluded from these considerations. Moreover, the majority of red algae producing high levels of EPA are macrophytic and can be excluded as well. But the euglenophyte Astasia longa SAG 1204-18 and chlorophyte Chlorococcum novae-angliae SAG 5.85 have a high DHA content of $15.4 \%$ of total FA and $16.1 \%$ of total FA (Tab. 2) and may therefore be promising candidates for further investigations. In addition the red alga Dixoniella grisea SAG 39.94 and the green alga Palmodictyon varium SAG 3.92 contain high amounts of EPA (37\% of total FA) and AA (74 \% of total FA), respectively (Tab. 3 and 4). A maximal accumulation of the desired VLCPUFAs may be traced by varying the growth conditions, like light, temperature and composition of the medium. 
Moreover it is important to know whether VLCPUFAs are present predominantly within membrane lipids or within TAGs (Sijtsma and de Swaaf 2004). The accumulation of VLCPUFAs within TAG would be beneficial for oil production, since the oil is most favourable for the use in nutritional applications. Furthermore it would indicate not only the existence of a biosynthetic pathway for VLCPUFAs, but in addition the existence for TAG specific acyltransferases, which might be useful for plant engineering.

It is speculated, that microalgae growing under extreme conditions, like rapid temperature changes and high intensity of light and UV radiation, have evolved a mechanism to rapidly change the VLCPUFA content of the membranes (Cohen and Khozin-Goldberg 2005). The storage of VLCPUFAs in TAG would allow the alga to adapt swiftly to the rapidly changing environment. Recently the green microalga Parietochloris incisa was isolated from the extreme habitat Mt. Tatyama in Japan and was found to be the richest known plant source of the VLCPUFA AA (Bigogno et al. 2002). P. incisa is capable to accumulate AA in high quantities within TAG ( $43 \%$ of total FA in the stationary phase) and with changing the growth conditions, like nitrogen-starvation, the AA content can even be increased up to $59 \%$ of total FAs (Cohen and Khozin-Goldberg 2005). The maximal accumulation of AA in TAG was reached when $P$. incisa was grown under optimal growth temperature of $25^{\circ} \mathrm{C}$, at relatively high biomass concentration and especially under nitrogen starvation (Cohen and Khozin-Goldberg 2005). Sudden cooling to low temperatures lead to a transfer of AA from TAG to polar lipids, indicating the role of TAG as a depot of VLCPUFAs, which can quickly be mobilised and incorporate into membranes under extreme conditions (Cohen and Khozin-Goldberg, 2005). Though, $P$. incisa may be a suitable candidate for large scale production of AA for human nutrition as well as for production of high purity AA for pharmaceutical purposes.

In contrast, the closely related microalgae Myrmecia bisecta SAG 2043 and M. incisa did not show this high accumulation of AA in TAG (Reinecke 2006). Although the entire FA profiles were similar, an increase of AA content upon nitrogen-starvation was not observed. However, the growth conditions and experimental procedures were different in comparison to the work of Bigongo et al. (2002), which could explain the different outcome of the investigations. In addition, first data were obtained, concerning the accumulation of VLCPUFAs in TAG the already mentioned red microalga D. grisea SAG 39.94 (Reinecke 2006). An increase of VLCPUFAs, in particular EPA, within TAGs and glycolipids was observable under nitrogen-starvation and with growing culture age. Therefore $D$. grisea was proposed to be a suitable alternative EPA producer in comparison 
to e.g. Nannochloropsis gaditana, which has already demonstrated its production potential of EPA (Reinecke 2006).

\section{Engineering VLCPUFA biosynthetic pathways into plants}

Metabolic engineering of oilseed crops leading to the reconstitution of PUFA synthesis has become a challenging alternative technique. Since microalgae are the primary producers of PUFAs they are also a good source for genes encoding the biosynthetic enzymes of VLCPUFAs (Kinney 2006). There are two types of metabolic pathways found among micro-organisms. First the standard FA biosynthetic pathway found in eukaryotes consisting of desaturation and elongation reactions and second the biosynthesis of FAs via PKSs (Metz et al. 2001; Napier and Sayanova 2005). Several studies have been published, reporting on implementation of algal desaturases and elongases into plants leading to the production of VLCPUFAs in Arabidopsis and linseed (e.g. Qi et al. 2004, Abbadi et al. 2004, Wu et al. 2005). The optimisation of VLCPUFA production within plants as well as the search for new enzymes facilitating the synthesis of VLCPUFAs and the bypass of bottlenecks is the focus of many current studies (Napier 2007).

The database obtained in this thesis provides information about microalgae producing VLCPUFAs in high levels. This information can be used to find enzymes involved in the biosynthesis of PUFAs with new properties. As mentioned before the chlorophyte P. varium SAG 3.92 was found to produce AA in high amounts (Tab. 4). A closer look to its FA profile shows that beside $16: 0$ and LA with a relative proportion $>5 \%$ of total FAs, AA was the major FA with $74 \%$ of total FAs $(102.3 \mu \mathrm{g} / \mathrm{mg}$ d.w.). The unusual high amount of AA and in parallel the low concentration of other FAs leads to the hypothesis that an enzyme related to PKS may be responsible for the biosynthesis of AA in P. varium. Normally, PKS form a very large enzyme complex with a molecular weight of more than 200.000, which catalyses the iterative elongation and desaturation of growing acyl-ACP chains, whereas intermediates are not released (Bentley and Bennett 1999). Recently, a complete PKS-type DHA synthase was cloned and characterised from the thraustochytrid marine protist Schizochytrium (Metz et al. 2001). Feeding experiments with labelled acetate showed that Schizochytrium produced DHA within one minute. Further experiments supported the idea that this protist produces DHA via a PKS related pathway and not via membrane-bound desaturases and CoA-specific FA elongases. The authors discussed that the identification of PUFA PKSs provide new tools to engineer the production of certain PUFAs in oilseed crops. 
Thus, it would be worthwhile to characterise the FA metabolism of $P$. varium in a detailed manner, in order to confirm the hypothesis that $P$. varium produces AA via the PKS pathway.

Based on the large screen several dinophycean microalgal strains were identified, which produce EPA and DHA in high proportions. As mentioned before the cultivation of dinophytes in a large scale is technically demanding and therefore these algae are not suitable for industrial VLCPUFA production. Nevertheless, dinophytes may serve as a gene source for enzymes involved in VLCPUFA biosynthesis and their transfer into TAG. The microalgal strain Cystodinium sp. SAG 59.97 was cultivated and its lipid content analysed (experiment was performed once, data not shown). Preliminary data showed that with growing culture age Cystodinium sp. accumulated large oil bodies, which were visible under the light microscope. Furthermore the data obtained from lipid analysis showed, that EPA and DHA were predominantly present in glycolipids and TAG. Therefore Cystodinium but also other dinophytes may be promising candidates to isolate genes encoding specific desaturases, elongases and acyltransferases of the VLCPUFA biosynthesis pathway. The construction of cDNA libraries for the dinophycean algae Lingulodinium polyedrum (Bachvaroff et al. 2004), Alexandrium tamarense (Hackett et al. 2005) and Amphidinium carterae (Bachvaroff et al. 2004) may facilitate the finding of genes encoding enzymes involved in VLCPUFA biosynthesis.

The understanding of acyl trafficking between the phospholipid and acyl CoA pool is still rather low and the clarification of this mechanism as well as the isolation of enzymes involved in this process is needed (Truksa et al. 2006). Since the alternating steps of lipidlinked desaturation and CoA-linked elongation appears to be a limiting factor in VLCPUFA synthesis, the use of CoA-dependent desaturases would bypass this problem (Napier 2007). So far only one desaturase using acyl-CoAs instead of lipid-linked substrates was isolated from non animal origin, namely the prasinophycean alga Ostreococcus tauri (Domergue et al. 2005). All other known CoA-dependent desaturases originate from animals, but transgenic plants expressing animal genes may have a negative impact on the already sensitive issue of public acceptance of genetically modified food (Napier 2007). Furthermore the heterologous expression of genes originating from different, often very distant, organisms may result in an inefficient transfer of substrates between the involved enzymes (Truska 2006). Hence, the identification of more CoAdependent desaturases from the plant kingdom is needed, but the low genome sequence information available for algae and plants makes the isolation of these genes difficult. 


\section{Fatty acid profile as chemotaxonomical marker for microalgae}

The lack of morphological characters makes the delimitation of most microalgal species difficult (Lang 2003; Müller 2006). To circumvent this disadvantage, biochemical and physiological characters have been used to help to unravel microalgal taxonomy. Molecular markers provided a new type of data that may be used to test hypotheses concerning the classification of microalgae based on morphological, physiological and biochemical characters. At present the most prominent applications are so-called fingerprint techniques like amplified fragment length polymorphism (AFLP) (Vos et al. 1995) and the sequencing of ribosomal DNA (Woese and Fox 1977) and plastidial genes (e.g. Yoon et al. 2004). Fig. 35 illustrates the molecular timeline for the origin of photosynthetic eukaryotes, meaning the evolutionary relationship of the main algal groups, rhodophytes, chlorophytes, stramenopiles, cryptophytes and haptophytes (Yoon et al. 2004).

Many studies suggested a causative relationship between endosymbiosis theory of microalgae evolution and FA composition for Chlorophyceae, Eustigmatophyceae, Bacillariophyceae and Dinophyceae (Sargent et al. 1995). It was found, that the relative proportion and composition of FAs in microalgae is characteristic for every species and genus, but is also dependent on the environmental conditions (Napolitano et al. 1997; Zhukova and Titlyanov 2003; Teoh et al. 2004). Viso et al. (1993) determined FA profiles of 28 marine microalgae from nine taxonomic classes and they were able to define species dependent FAs or FA ratios. Moreover they found a profile, which was roughly taxon specific when the cells were cultured under identical growth conditions. 


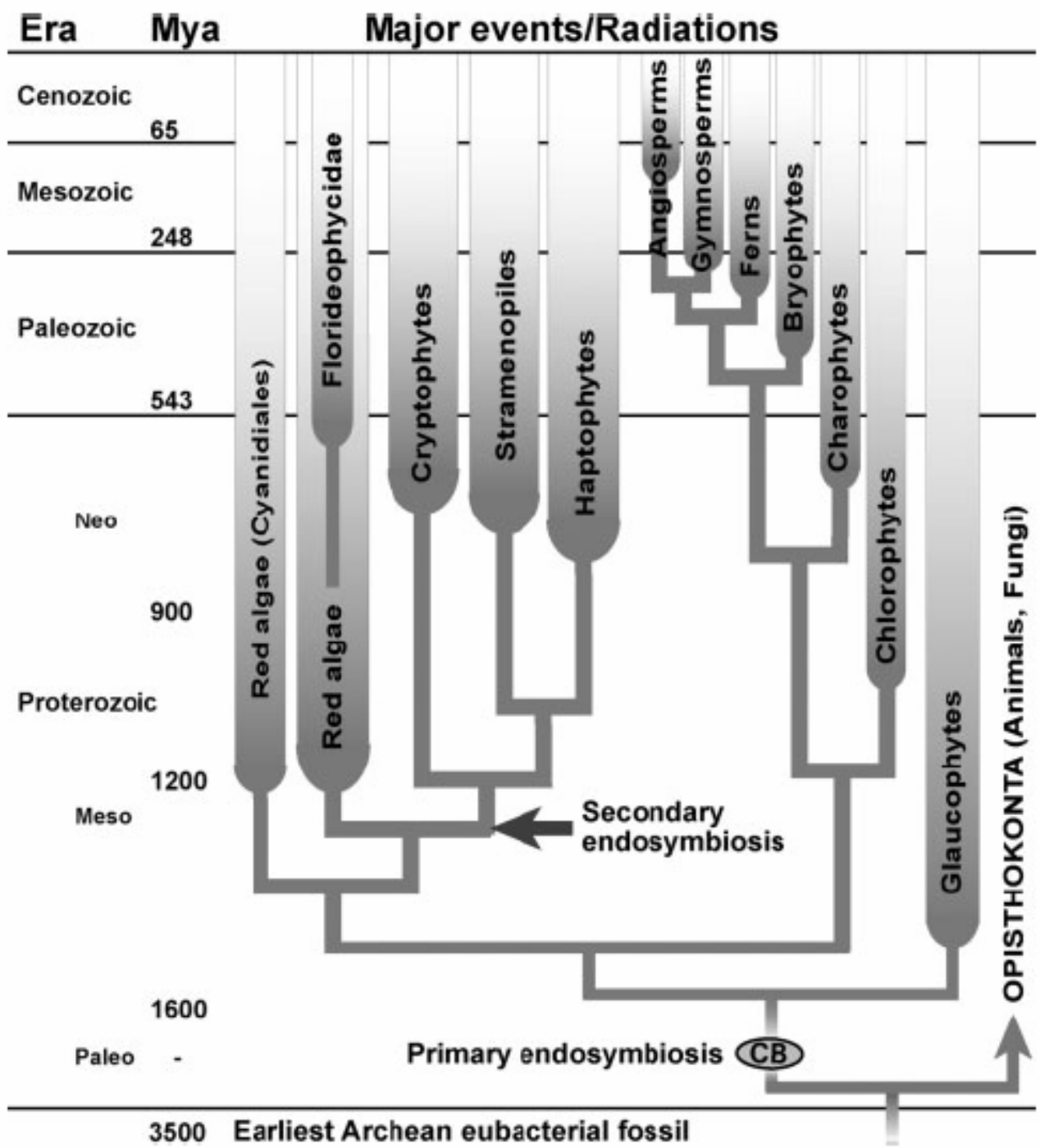

Figure 35. Illustration of evolutionary relationship and divergence times for red, green, glaucophyte and chromist (chlorophyll-c-containing cryptophytes, stramenopiles, and haptophytes) algae. CB, cyanobacteria (from Yoon, 2004)

According to the FA composition of microalgae from the green algal classes Chlorophyceae and Prasinophyceae Dunstan et al. (1992) defined two distinct groups. Species from the Prasinophyceae contained significant proportions of C20 and C22 VLCPUFAs whereas species from the Chlorophyceae contained high amounts of C-16 and C-18 PUFAs. Leblond et al. (2005) studied the lipid composition of genera belonging to the class of Chlorarachniophyceae. Their unusual morphology and physiology lead to the hypothesis that they evolved as a result of a secondary endosymbiosis, where a green alga, in particular Chlorella, was engulfed by a heterotrophic amoeba (Hibberd and Norris 1984; Ludwig and Gibbs 1989; Cavalier-Smith 1999; Gilson 2001). Therefore the authors expected similarities in the FA composition of the glycolipids in the chloroplasts of 
Chlorarachniophyceae and green algae. However, the characterisation of the lipids showed that some FAs occurring in the glycolipids of Chlorarachniophyceae have never been found in any species of Chlorella, which raised new questions about the evolutionary origin of the chloroplast in Chlorarachniophyceae (Leblond et al. 2005).

Furthermore some studies focus on the possibility to use FAs and sterols as biomarkers, in organic geochemical studies (Volkman et al. 1998). Especially the characterisation of sterol profiles was suggested to be useful as evolutionary marker (Leblond et al. 2005). Even the algal origin of the non photosynthetic micro-organisms Prototheca wickerhamii and Dictyostelium discoideum was assumed after the analysis of their sterol compositions (Nes et al. 1990).

First attempts of comparing the FA profile of different SAG strains, showed, that the profiles were very similar among strains of one species, but the profiles of different taxonomic algal groups like Rhodophyceae, Cyanophyceae, Chlorophyceae and Euglenophyceae were obviously different. Some microalgae contain high amounts of certain VLCPUFAs such as EPA and DHA, whereas others contain high amounts of saturated and monounsaturated FAs.

For example one characteristic feature of most strains of the Rhodophyceae is their high amount of AA and EPA ranging from $10.2-54.2 \%$ of total FAs and $10-67 \%$ of total FAs, respectively (III-1.2). This has been also described in the literature, e.g. the red microalga Porphyridium cruentum is known for its potential of producing AA and EPA in high levels (Cohen et al. 1988; Cohen 1990). Furthermore, Dinophyceae have high levels of EPA and DHA and some contain the unusual PUFA 18:5n-3. Other classes of microalgae contain high levels of all three VLCPUFAs DHA, EPA and AA, e.g. Eustigmatophyceae, Cryptophyceae, Euglenophyceae and Haptophyceae. These results nicely correspond to data obtained in similar studies, where algae from different classes were analysed for their FA profile (Viso and Marty 1993; Shiran et al. 1996; Tonon et al. 2002; Dunstan et al. 2005).

Furthermore, Chlorophyceae, Trebouxiophyceae and Streptophyceae rarely contain significant amounts of AA, EPA and DHA, but instead have a predominance of C16 and C18 PUFAs, like roughenic acid, 16:4 ${ }^{(4 Z, 7 Z, 10 Z, 13 Z)}$, LA and ALA. This characteristic FA composition is also found in flowering plants. Since green algae are regarded as the ancestors of flowering plants (Fig. 35, (Cavalier-Smith 1986) it is likely that they share similar FA compositions. Nevertheless there are some trebouxiophytes and chlorophytes harbouring unusual high levels of AA and DHA. Palmodictyon varium SAG 3.92, 
Trochisciopsis tetraspora SAG 19.95 and Myrmecia bisecta SAG 2043 showed high amounts of AA of about $40-74 \%$ of total FAs, whereas Chlorococcum novae-angliae SAG 5.85 contains a high level of DHA. As described before, another exception is the trebouxiophyte $P$. incisa, which was found to be the richest plant source of AA so far, since it accumulates this VLCPUFA to amounts of about $59 \%$ of total FAs (Bigogna et al. 2002).

The data obtained for the cyanobacteria show, that they can roughly be subdivided to their ability to produce C18 PUFAs or not. A comparison with data obtained by (Rippka et al. 1979) showed that FAs profiles of several strains belonging to the five main orders revealed interesting results (Ikawa 2004). All cyanobacteria, except Nostocales, appeared to be divided between PUFA producer and non producer. Based on 16SrRNA data, for at least three orders (Stigonematales, Oscillatoriales and Chlorococcales) the polyphyletic nature was described before (Honda et al. 1999; Litvaitis 2002). In the past several studies indicated the importance of FA composition in the taxonomy of cyanobacteria at the genus and subgenus level (Caudales and Wells 1992; Kruger et al. 1995). It could even be shown, that the hydrocarbon and FA composition can be used as chemotaxonomical marker at the species level (Dembitsky and Srebnik 2002; Rezanka et al. 2003).

Temina et al (2006) focused on the genus Nostoc. The authors investigated the chemotaxonomic and phylogenetic relationships among 6 species of the genus from different habitats by analysing low molecular hydroxy, idiocy, saturated and unsaturated FAs. The data obtained were applied to cluster analysis in order to analyse the chemotaxonomic relation and interpret the FA content of species of the genus Nostoc.

They found that all samples from the same original population exhibited characteristic FA compositions, leading to the conclusion that the FA composition of the different strains of Nostoc is rather under environmental than under genetic control (Temina et al. 2006). In contrast to these results, the comparison of FA profiles of red, brown and green algae from different regions of the world showed that algae had similar FA profiles typical for each taxonomic group independently of the geographical source (Khotimchenko et al. 2002).

Hence, additional investigations of more strains from different habitats are needed to examine the relation between FA profile, natural environment and phylogeny.

Microalgae which are important in marine food webs, including representatives of the classes Bacillariophyceae, Prasinophyceae and Raphidophyceae are well investigated with respect to their lipid and FA composition. Moreover some species are used successfully as 
microalgal feed stocks for larval animals. Hence, a comparison between FA profiles published for these microalgal species and the FA profiles obtained in the present work is possible.

In the present screen the FA content of 22 strains of the class Bacillariophyceae, representing 11 genera and 16 species, have been analysed. In general, the major FAs of these algae were $16: 0,16: 1^{(9 Z)}$ and EPA, which is confirmed by data published about detailed examinations of lipid and FA composition of several diatom species (Dunstan et al. 1994; Alonso et al. 1998). The diatom Phaeodactylum tricornutum has been intensively investigated for its EPA production potential (Wen and Chen 2005) and therefore several reports on its FA profile are available. Three strains of $P$. tricornutum are maintained in the SAG and show similar FA profiles. The main FAs are 16:0 (ranging from $8.4-13.6 \%$ of total FA), 16:1 ${ }^{(9 \mathrm{Z})}$ (ranging from $37.1-41 \%$ of total FAs) and EPA (ranging from 13.3 $23.4 \%$ of total FAs). The comparison with data published by Tonon et al. (2002) and Alonso et al (1998) show that the overall FA profile of P. tricornutum CCAP 1052/1a and P. tricornutum UTEX 640 is similar to that of the SAG strains and the relative proportions of the main FAs 16:0, 16:1 ${ }^{(9 \mathrm{Z})}$ and EPA in the stationary phase are almost the same.

Comparing the FA profile of the prasinophycean microalga Mircomonas pusilla SAG 39.85 with data published (Dunstan et al. 1992) shows that the FA profiles were rather different. Whereas the main FAs of the SAG strain are 16:0 (13.9\% of total FAs), 16:1 ${ }^{(9 \mathrm{Z})}$ (10.4 \% of total FAs) $16: 4 n-3$ (7.6\% of total FAs) and 18:0 (43\% of total FAs) the $M$. pusilla strains CS-98 and CS-170 contain high proportions of 16:0 (13.6 - 20.6\% of total FAs), $16: 4 n-3$ (15.5 - $20.4 \%$ of total FAs), 18:4n-3 (20.7-26.3 \% of total FAs) and 18:5n-3 (10.6-16.7 \% of total FAs). The latter was not even present in M. pusilla SAG 39.85. The discrepancy of the FA composition of different M. pusilla strains may be explained by the use of different culture conditions and the culture age, since the data published were obtained from algae in the exponential phase and not in the stationary phase.

The most abundant FAs of the raphidophycean alga Heterosigma akashiwo were 16:0, 16:1 ${ }^{(9 Z)}, 18: 4 n-3$ and EPA (Nichols et al. 1983; Viso and Marty 1993). In comparison, the same major components were found for the SAG strain H. akashiwo SAG 21.86. Although H. akashiwo is describe to produce 18:5n-3 it was not detected for the SAG strain in this present study. This may again be explained by the different growth conditions and the culture age. 
The database may further be used to elucidate chemotaxonomy among algae. A combination of several criteria can be selected to discriminate taxonomic groups. Despite the overall FA profile of representatives of different genera or classes, the ratio of number of carbon atoms and double bonds can be studied, meaning the ratio of e.g. C16 FAs/C18 FAs or C16 PUFAs/C 18 PUFAs 18:5n-3/18:3n-3 etc. (Viso and Marty 1993). Furthermore the presence or combination of certain FAs can be a characteristic for particular algal classes (Volkman et al. 1998).

First efforts were made to find a biological marker, in particular a certain FA which may characterise a group of algae. Only PUFAs with a significant proportion $(>10 \%)$ of the total FAs content were taken into consideration. In the following section the use of EPA, pinolenic acid and 18:5n-3 as chemotaxonomical markers are discussed exemplary.

\section{The use of EPA as a chemotaxonomical marker}

The VLCPUFA EPA was found in a broad range of different algal classes (Fig. 10). Most representatives harbouring EPA in higher proportions than $10 \%$ of total FAs belonged to the class Rhodophyceae and Xanthophyceae (Fig. 36). But high proportions of EPA are also described for several species belonging to Bacillariophyceae (diatoms) and Eustigmatophyceae and Rhodophyceae (Cohen 1990; Dunstan et al. 1994; Sukenik 1999). Taking into account that the present screen includes fewer representatives of Eustigmatophyceae and Bacillariophyceae than of Rhodophyceae and Xanthophyceae limits the use of EPA as a biomarker for a certain algal group. Furthermore too many different algal classes provide high potential for EPA production, making chemotaxonomic analysis even more complicated. 


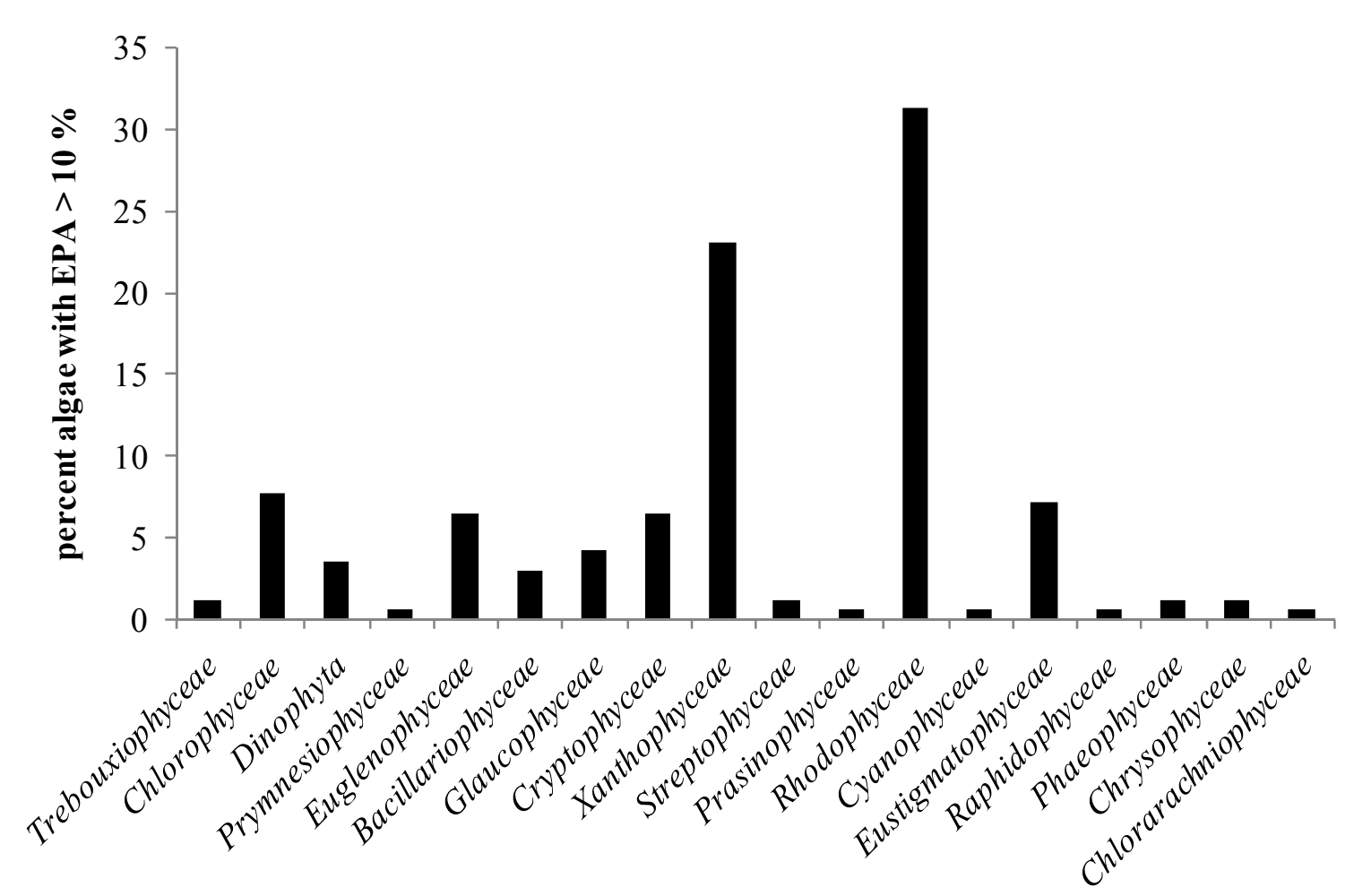

Figure 36. Microalgal classes producing EPA in higher proportions than $10 \%$ of total FAs. Each bar represents the amount of algae of one class in percent of algae producing EPA in high amounts.

The use of pinolenic acid as chemotaxonomical marker for certain chlorophytes

Pinolenic acid $\left(18: 3^{(5 Z, 9 Z, 12 Z)}\right)$ is a $\Delta 5$-unsaturated bis-methylene-interrupted FA, commonly found in the seed lipids of gymnosperm pines. Giroud et al. (1988) showed that pinolenic acid is not restricted to gymnosperm oil, but is also present in betaine and phospholipids of the chlorophyte Chlamydomonas reinhardtii. The analysis of lipids from C. reinhardtii revealed, that pinolenic acid is one of the major FAs of the betaine lipid DGTS and the desaturation of FAs was shown to be lipid-linked with a preference for the sn-2 position (Giroud and Eichenberger 1989). Since pinolenic acid is discussed to have beneficial physiological effects in animals (Wolff and Christie 2002) the recent isolation of the 113 desaturase from C. reinhardtii contributes the development of transgenic plants harbouring this oil (Kajikawa et al. 2006). In the present work, the majority of microalgae producing pinolenic acid are assigned to chlorophytes (94\% of $\Sigma$ algae containing pinolenic acid), in particular to the genus Chlamydomonas and Chlamydocapsa (Fig. 11, Tab. 8). Though, it is tempting to suggest pinolenic acid as a useful marker for these certain chlorophytes. Moreover pinolenic acid might be a useful marker for the detection of betaine lipids, since 
published data showed that pinolenic acid is exclusively linked to PE and DGTS (Giroud 1988, Giroud Eichenberger, 1989).

\section{The use of 18:5n-3 as a chemotaxonomical marker for marine phytoplankton species}

The unusual FA 18:5n-3 was found to be likely characteristic for certain algae groups. In this work, only strains of the class Dinophyceae and Haptophyceae contained 18:5n-3 in significant amounts (Tab. 5, Fig. 37). The high abundance of 18:5n-3 in some dinophytes and haptophytes has been reported before (Joseph 1975; Mayzaud 1976; Volkman et al. 1981). Subsequently microalgae of the class Raphidophyceae were identified for synthesising this FA, too (Nichols et al. 1987; Bell et al. 1997). But the bulk of 18:5n-3 is restricted to the marine ecosystem and is likely to be contributed by dominant toxic bloom species, like Prymnesium parvum and Gymnodinium cf. mikimotoi. Since dinophytes, haptophytes and raphidophytes evolved independently during secondary and tertiary endosymbiosis (Fig. 35, Yoon and Hackett, 2005), a common ancestor with the ability to synthesise 18:5n-3 may be excluded. But on the other hand, all three classes evolved by engulfment of a rhodophycean alga by a heterotrophic unicellular organism (Fig. 35, Cavalier-Smith 1999, Yoon and Hackett, 2005) and therefore the genes responsible for 18:5n-3 synthesis may derive from this symbiont. The fact, that at least in one rhodophyte low proportions of 18:5n-3 were found, may support this hypothesis. At this point it should be emphasized that the data obtained represent just one measurement per strain and should be confirmed by at least a second screening.

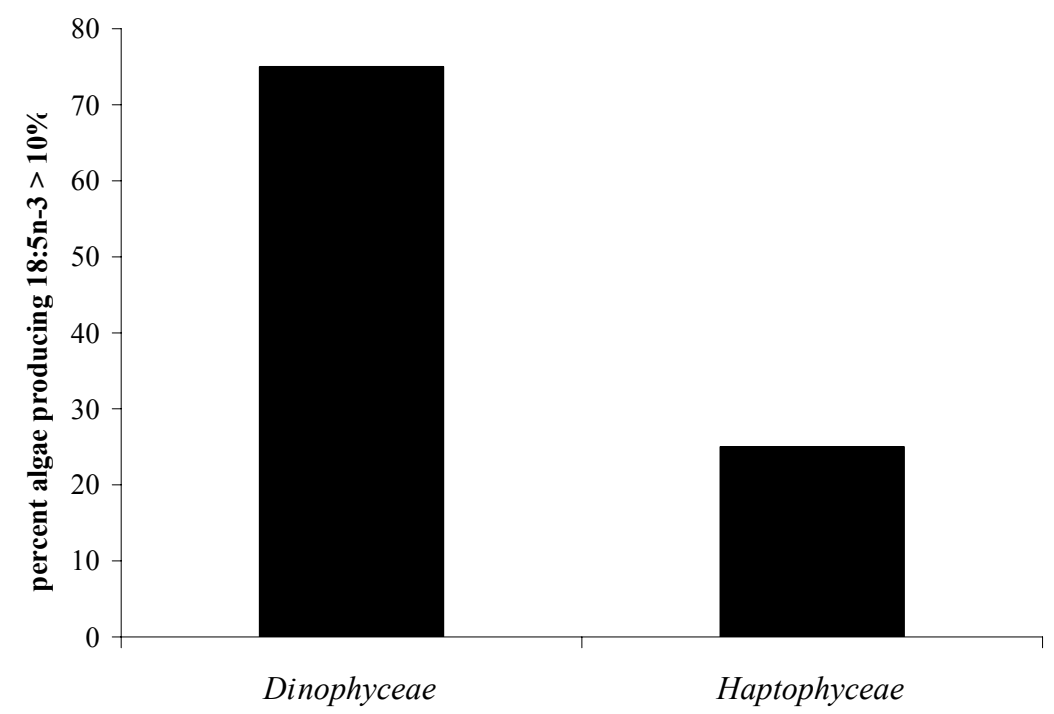

Figure 37. Microalgal classes producing 18:5n-3 in proportions $>10 \%$ of total FAs. Bars represent percent algae of all algae producing EPA in proportions $>10 \%$. 


\section{Analysis of biosynthesis of $18: 5 n-3$ in $P$. parvum as a prerequisite for gene isolation}

The VLCPUFAs AA, EPA and DHA are proven to be beneficial for human health and much interest is focussed on the identification and characterisation of organisms that produce these VLCPUFAs and to identify and isolate the corresponding biosynthetic pathway in order to implement it into higher plants. The identification of microalgal strains harbouring AA, EPA and DHA in high proportions was only one aim of the present work. Furthermore it was concerned with identifying microalgae, producing unusual FAs. The term unusual" refers to FAs having an unusual number or position of double bonds or other functional groups like methyl s or hydroxyl groups etc. One interesting FA is $18: 5 n-3$ due to its high number of double bonds in the carbon chain and the terminal double bond three carbons proximate to the carboxyl terminus. 18:5n-3 was first identified in marine photosynthetic dinoflagellates (Joseph 1975), and since then further studies reported about the occurrence of 18:5n-3 in Haptophyceae and Raphidophyceae (e.g. Volkman et al. 1981, Bell et al. 1997). Moreover it was discussed to be a putative chemotaxonomical marker for a restricted group of marine microalgae (Dinophyceae, Haptophyceae and Raphidophyceae; Mayzaud 1976 and Volkman et al 1981). Since 18:5n-3 is present in large proportions in algal species and may be responsible for ichthyotoxicity, it is considered to be linked to fish death during toxic blooms in seawaters (Yasumoto et al. 1990). Arzul et al. 1995 demonstrated, that high concentrations of 18:5n-3 and DHA produced by the dinophyte Gyrodinium $c f$. aureolum inhibited the growth of the diatom Chaetoceros gracile. The toxic effects of 18:5n-3 were also supported by showing that 18:5n-3 inhibits ATPase activity accompanied by a bronchial morphological change in the gills and intestine of the sea bass Dicentrarchus labrayx (Sola et al. 1999). Furthermore it was shown, that 18:5n-3 was responsible for an inhibition or a delayed first cleavage stage of sea urchin eggs (Sellem et al. 2000).

Although the toxicity of 18:5n-3 in high concentrations was proven, the question remained open, if animals are able to metabolise 18:5n-3 when they ingest phytoplankton. Ghioni et al. 2001 prepared 18:5n-3 from the haptophycean alga Isochrysis galbana and studied its metabolism in cultured cells from turbot, seabream and Atlantic salmon. All three species converted all-cis 18:5n-3 to $18: 4 n-3$ via sub sequential isomeration, forming a 2-trans 18:5n-3 intermediate and further hydrogenation leading to $18: 4 n-3$. The latter was then elongated and desaturated to EPA. According to this study, it would be interesting, to investigate the metabolism of 18:5n-3 supplied to mammalian cells. Since fish cells 
efficiently convert 18:5n-3 to EPA, this PUFA might display an alternative supplement for human diet.

However, the biosynthetic pathway of 18:5n-3 in microalgae is still not clarified. Joseph (1975) proposed two routes by which this FA might be synthesised, either through desaturation of 18:4n-3 or in analogy with the Sprecher pathway (Sprecher 2000) by the loss of an acetate unit from EPA by partial peroxisomal $\beta$-oxidation.

In order to produce 18:5n-3 in flowering plants, one needs to know the enzymes involved. Therefore it was aimed to elucidate the biosynthetic pathway of 18:5n-3 by analysing first the localisation of $18: 5 n-3$ in the different lipid classes and than by analysing the fate of exogenously fed labelled FAs in the haptophycean alga $P$. saltans. Lipid analysis revealed that 18:5n-3 was linked to neutral, glyco and phospholipids and additionally to the betaine lipid DGTS. Therefore 18:5n-3 is not restricted to membrane lipids of the plastids, but appears also in extraplastidial membrane and storage lipids. The total amount of phospholipids was rather low and instead three different betaine lipids were observed by TLC. Due to missing standards only DGTS was clearly identified, whereas the other two bands are considered to represent the betaine lipids DGCC and DGTA (Fig. 14). Betaine lipids mainly occur in higher plants and algae, like Chlamydomonas reinhardtii (Eichenberger 1976) and Chlorella minutissima (Haigh et al. 1996), but also in representatives of other classes including Chrysophyceae and Haptophyceae (reviewed by (Dembitsky 1996). Thus, with P. saltans another haptophyte was identified for producing betaine lipids.

Due to their zwitterionic structure betaine lipids resemble the structure of PC (Fig. 38) and many studies showed that algae containing betaine lipids do not contain detectable amounts of PC (Giroud et al. 1988, Kato et al. 1996). Therefore it is assumed, that betaine lipids take over the functions of PC, at least in organisms lacking PC. Subcellular localisation experiments revealed, that DGTS is mainly synthesised via the eukaryotic pathway (Eichenberger 1993). The examination of FAs linked to betaine lipids in several microalgae and plants supports the cytoplasmic origin of DGTS (Sato and Furuya 1983; Giroud et al. 1988). The haptophyte Pavlova lutheri contains DGCC and DGTA exclusively of eukaryotic structure with high amounts of EPA and DHA at the sn-2 positions (Eichenberger and Gribi 1997). The betaine lipids of P. saltans lack EPA and DHA but contain high amounts of 16:0, 18:0 and 18:5n-3 (Tab. 12). The data have to be verified by further lipid analysis, including analysis of positional distribution of FAs. 
Recently, Riekhof et al. (2005) isolated and characterised a betaine lipid synthase BTA $1_{\mathrm{cr}}$ from C. reinhardtii, which is involved in the biosynthesis of DGTS. First insights into the biosynthesis of betaine lipids and their possible role in lipid metabolism were obtained by studies of Vogel and Eichenberger 1992 and Haigh et al. 1996. However, the overall function and distribution of betaine lipids in algae and plants is still unknown.

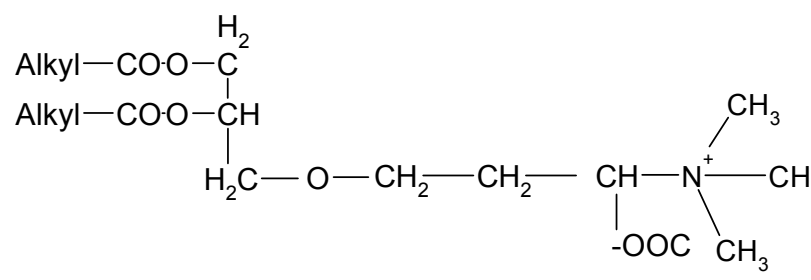

DGTS<smiles>C[N+](C)(C)CC(COCC(COC(N)=O)C(=O)O)C(=O)O</smiles>

DGTA<smiles>C[N+](C)(C)CCOC(COC(COC(N)=O)C(N)=O)C(=O)O</smiles>

DGCC<smiles>C[N+](C)(C)CCOP(=O)([O-])OCC(COC(N)=O)OC(N)=O</smiles>

PC

Figure 38. Comparing structures of betaine lipids and PC (from Dembitsky, 1996)

The elucidation of the biosynthetic pathway of 18:5n-3 was further analysed by feeding labelled FAs to P. parvum. At the beginning two possible routes of 18:5n-3 biosynthesis were proposed as mentioned above. To exclude the possibility of synthesis of 18:5n-3 through partial $\beta$-oxidation of EPA the algal cultures were either grown in presence of $\left[\mathrm{U}_{-}{ }^{13} \mathrm{C}\right]-\mathrm{LA}$ or of $\left[19,19,20,20,20-\mathrm{D}_{5}\right]$-EPA (III-2.5). When algal cultures were incubated with [U- $\left.{ }^{13} \mathrm{C}\right]-\mathrm{LA}$ labelled 18:5n-3 was detected in the total lipid extracts from the cells. However, labelling was also detected in 18:4n-3 and its elongated and further desaturated product EPA. In contrast, when cells were incubated with $\left[19,19,20,20,20-\mathrm{D}_{5}\right]-\mathrm{EPA}$ no labelled 18:5n-3 was detectable, but labelled 22:5n-3 and DHA instead. Thus the lack of $\mathrm{D}_{5}-18: 5 \mathrm{n}-3$ in total lipid extracts of algae incubated with $\left[19,19,20,20,20-\mathrm{D}_{5}\right]-\mathrm{EPA}$ 
indicates that this FA is synthesised via desaturation of $18: 4 n-3$. This in turn would implicate the existence of a so far unknown $\Delta 3$-desaturase which may insert the last $(\Delta 3)$ double bond from 18:4n-3 to 18:5n-3 (Fig. 1). Taking into account, that the incorporation of externally feed FAs may not completely reflect the de novo synthesis (Khozin et al. 1997), it may also lead to an inhibition of enzymes involved in the biosynthesis of PUFAs and therefore $\beta$-oxidation of $\left[19,19,20,20,20-\mathrm{D}_{5}\right]$-EPA may not totally excluded. A regulation of gene expression by the addition of saturated or unsaturated FAs to the growth medium was shown for Saccharomyces cerivisiae (Choi et al. 1996). Saturated FAs induced transcription of the $\Delta 9 \mathrm{FA}$ desaturase gene, whereas the addition of unsaturated FAs repressed the transcription of the respective gene. The authors further discussed the importance of maintenance of FA composition in controlling the properties of membrane and storage lipids and suggested a diverse array of control mechanisms in the regulation of unsaturated FA formation (Choi et al 1996). Thus, comprehensive studies analysing the lipid and FA composition in presence and absence of labelled FAs are still powerful approaches to identify FA metabolising enzymes. 
Besides the identification of enzymes responsible for the biosynthesis of PUFAs the present work also includes the identification of oxylipin producing algae as well as enzymes responsible for the biosynthesis of oxylipins. Oxylipins comprise a large group of oxygenated compounds synthesised from FAs, which play important roles as signal molecules and mediators in animal and plant tissues but also in flavour and food industry. The formation of FA hydroperoxides is regarded as the initial step of oxylipin synthesis and is mainly catalysed by LOXs, respectively. Therefore LOXs display important enzymes for oxylipin production in biotechnology. In order to find new oxylipins and enzymes with unknown features involved in oxylipin synthesis, the alga collection was first screened for oxylipins and then available databases were searched for LOX sequences in microalgal genomes available. Three promising LOX sequences were found in the cyanobacteria Nostoc punctiforme and Nostoc sp. and the characterisation of these enzymes is discussed in the following section.

\section{LOX pathway of two Nostoc species}

Oxidation of PUFAs in flowering plants is mainly a controlled process that is catalyzed by enzymes of the LOX enzyme family (Liavonchanka and Feussner 2006). Products originating from the LOX reaction may be further metabolized in the so-called LOX pathway and are known as signalling substances that play a role in plant development as well as in plant responses to wounding and pathogen attack (Schilmiller and Howe 2005b; Wasternack et al. 2006). Apart from accumulating data in flowering, as well as from mammals and eukaryotic algae (Kühn 2005; Lotzer et al. 2005; Pohnert 2005b), information on the relevance of lipid peroxide metabolism in prokaryotic organisms is scarce (Müller 2004). Thus one aim of this work was to isolate and analyse LOXs and the corresponding oxylipin patterns from cyanobacterial origin, since these bacteria may be the ancestors for plastids in higher eukaryotes.

The analysis shows that several cyanobacterial genomes harbour putative open reading frames that may encode for a LOX. N. punctiforme PCC73102 and N. sp. SAG 25.82 were chosen as the most promising candidates, since their genome harbour different LOX enzymes. DNA isolated from these bacteria indeed yielded sequences for at least two different LOXs for $N$. punctiforme and a peroxidase-LOX fusion protein from $N$. sp. SAG 25.82 . 


\subsection{Two LOXs from $N$. punctiforme with interesting characteristics}

The two LOXs from N. punctiforme PCC 73102 were cloned from genomic DNA and named NpLOX1 and NpLOX2, respectively. Interestingly, NpLOX2 seems to be the smallest LOX protein of 584 amino acids isolated so far, since NpLOX1 and a homologous LOX from the bacterium Pseudomonas aeruginosa encode for proteins of 630 and 685 amino acids, respectively (Vance et al. 2004). Whereby mammalian LOXs do have about the same size of 660 to 680 amino acids, plant LOXs constitute normally of more than 860 amino acids (Feussner and Wasternack 2002). To date there is only one report describing a LOX of about the same size. It describes a proteolytic fragment harbouring the catalytic domain of soybean LOX1 of a weight about 60 having still LOX activity (Di Venere et al. 2003).

\section{Regiospecificity of NpLOX1 and NpLOX2}

Both NpLOX proteins were identified as linoleate 13-LOXs by expression in E. coli (Fig. 19, Tab. 13). In general, two different regioisomeres of C18 hydroperoxy PUFAs may be formed by selectivity in the site of oxygen insertion via rearrangement of the intermediate FA radical. The first step of the LOX reaction is the hydrogen removal from the methylene group between two double bonds resulting in a free radical. This leads in parallel to an oxidation of the iron atom $(\mathrm{Fe}(\mathrm{III}) \rightarrow \mathrm{Fe}(\mathrm{II}))$ in the catalytic site of the LOX. The resulting radical is further rearranged either to [+2] or [-2] position. And in the last step of the LOX reaction molecular oxygen is bound to the radical and a final proton adsorption leads to the hydroperoxide forming (Feussner and Kühn 2000). Consequently, when hydrogen is abstracted at C-11 in either LA or ALA, molecular oxygen can be introduced at position [+2] or [-2] leading to dioxygen insertion at C-13 or C-9 (Fig. 39).

In Fig 1.2 the stepwise LOX reaction is illustrated with LA as substrate. The resulting product of the conversion of LA can either be 13-HPODE or 9-HPODE. 


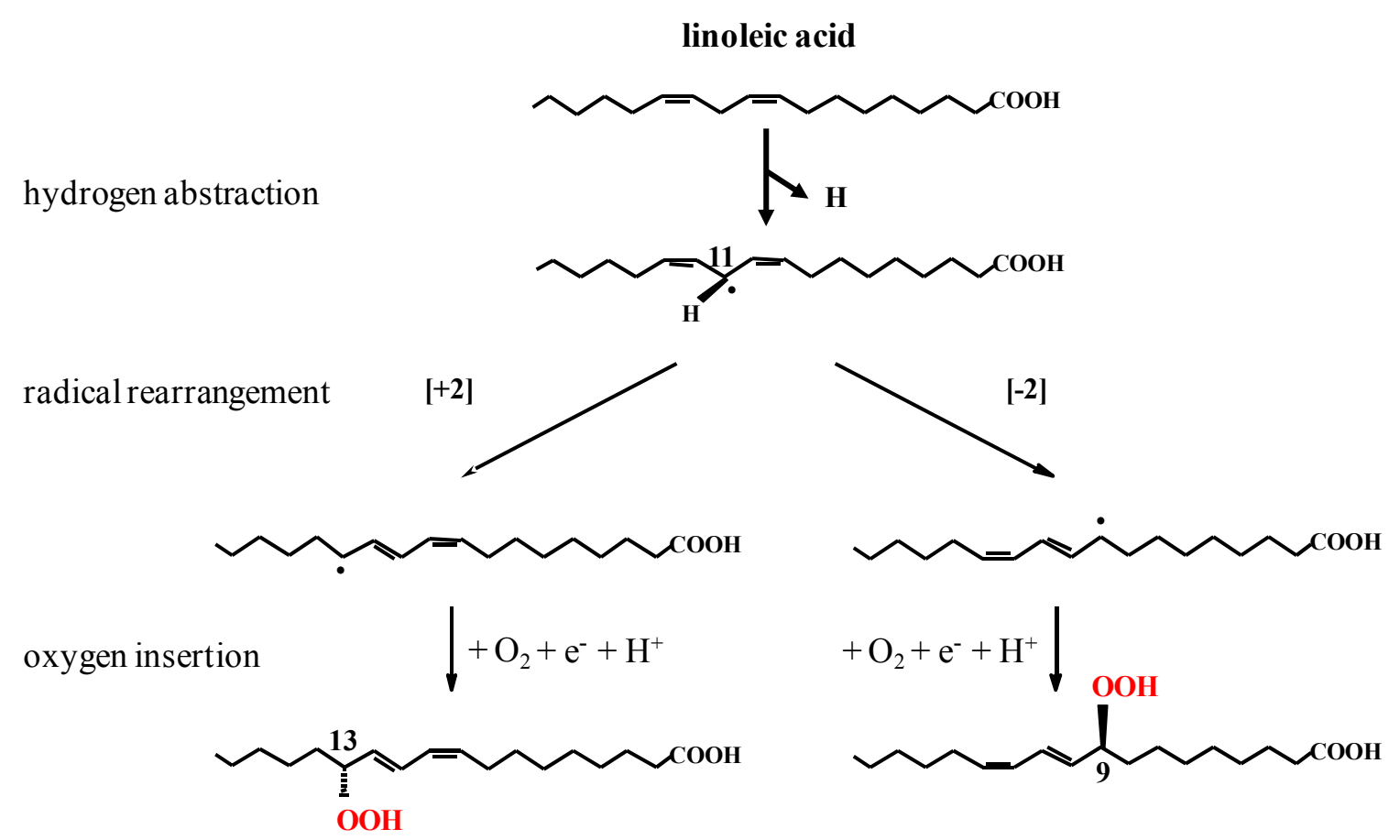

(13S)-HPODE

(9S)-HPODE

Figure 39. General scheme of the LOX reaction with LA as substrate. The reaction is divided into three steps: (1) the hydrogen abstraction, (2) the radical rearrangement and (3) the oxygen insertion (modified from Liavonchanka and Feussner 2006).

Furthermore two models are currently available to explain the underlying mechanism of positional specificity of LOXs (Fig. 40; Liavonchanka and Feussner 2006): Based on experiments with mammalian LOXs, a space-related hypothesis was established (Browner et al. 1998). In this model, the FA substrate penetrates the active site generally with its methyl end first. Then the depth of the substrate-binding pocket determines the positional specificity of molecular oxygen insertion. According to a second hypothesis, the substrate orientation is regarded as the key step in the determination of the position of dioxygen insertion: In the case of 13-LOXs, the active site is penetrated again by the substrate using its methyl end first, whereas with 9-LOXs the substrate is forced into an inverse orientation favouring a penetration with its carboxy group first (Gardner 1989). Consequently, a radical rearrangement at either [+2] or [-2], respectively, may be facilitated in both cases by the same mechanism within the active site. In addition, other factors can affect the regiospecificity of a LOX, like the $\mathrm{pH}$ or temperature (Feussner and Wasternack 2002). 


\section{Space-related model}

13-LOX

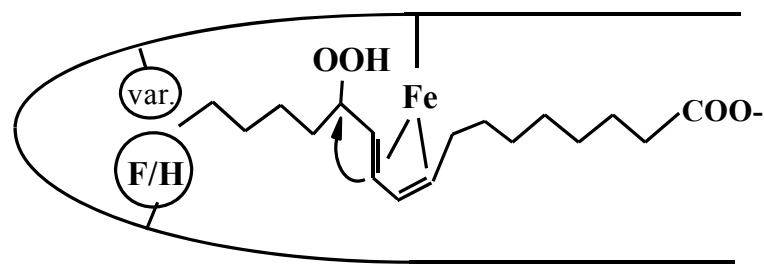

9-LOX

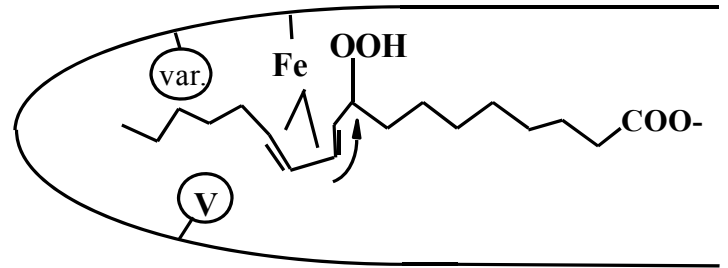

\section{Orientation-dependent model}

13-LOX

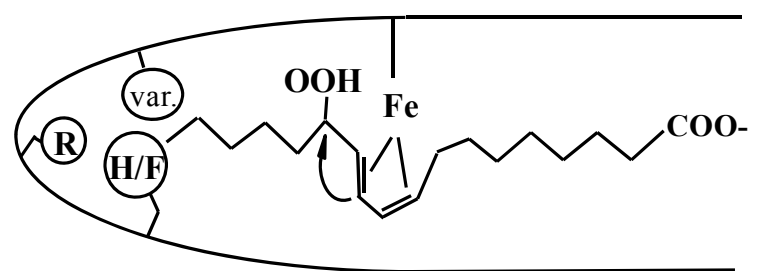

9-LOX

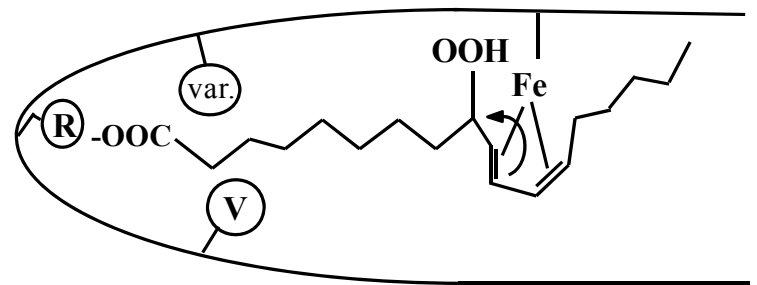

Figure 40. Models explaining the positional specificity of LOXs (according to Feussner and Wasternack, 2002)

In recent years a number of studies using site directed mutagenesis of amino acid residues in the active site of the enzyme have been carried out with mammalian LOXs (Kühn et al. 2005a). Here, two regions have been identified in the primary structure containing sequence determinants for the positional specificity: i) Amino acids aligning with the socalled Sloane determinant (Sloane et al. 1991). Interestingly, it is highly conserved among plant LOXs and seems to determine primarily their positional specificity. ii) Borngräber determinant: while this position seems to be important for mammalian LOXs, there is amino acid heterogeneity among plant LOXs at this position which aligns with P353 of the rabbit reticulocyte 15-LOX. However in mammalian 15- or 12-LOXs always a bulky amino acid is found at this position (Borngräber et al. 1996a). In addition, structural modelling of the active site of plant LOXs revealed that an arginine residue is localized in the vicinity of the putative substrate-binding pocket that seems to be a third determinant that is called Hornung determinant (Hornung et al. 1999a). It may interact with the carboxy group of the substrates when there is an inverse head to tail substrate orientation. In wildtype plant 13-LOXs there is a phenylalanine or a histidine located at a position which aligns with the Sloane determinant. These amino acids may shield the positive charge of the arginine and thus, the substrate may penetrate the active site with its methyl end since 
there is no counterpart to neutralize the charge of the carboxy group. Mutagenesis studies of the Sloane determinant with the lipid body LOX of cucumber seedlings supported this model (Hornung et al. 1999a). In contrast, for all plant 9-LOXs analysed so far, a small valine residue was identified at this highly conserved amino acid position. Structural modelling of the enzyme/substrate interaction suggests that small amino acid side chains cannot mask the positively charged guanidine group of the arginine residue at the bottom of the substrate pocket. As a consequence, the guanidino group may be able to form a salt bridge with the carboxylic group of the substrate, favouring an inverse head to tail substrate orientation (Liavonchanka and Feussner 2006). However, in most mammalian LOXs analyzed so far this determinant has not been found, rendering the orientation dependent model in mammalian LOXs unlikely (Kühn et al. 2005b). In summary, the space within the active site seems to be the main factor determining the positional specificity of mammalian LOXs whereas in case of plant LOXs the orientation of the substrate seems to be the major factor that determines the positional specificity.

In addition a fourth determinant was found, which seems to determine the stereospecificity of LOXs (Coffa et al. 2004). In $S$-LOXs it aligns with an alanine whereas in R-LOXs it aligns with a glycine. The Coffa determinant is conserved in NpLOX1 and 2 identifying both enzymes as $S$-specific LOXs. Most interestingly, at the level of the other 3 determinants (according to Sloane, Borngräber and Hornung) the active site of both enzymes is neither similar to mammalian LOXs nor to plant LOXs. Unlike in plant LOXs but similar to PpLOX1 from the moss P. patens (Senger et al. 2005b) an arginine at the bottom of the substrate binding pocket is absent and in contrast to mammalian 13-LOXs the amino acid side chains at the other two positions are rather small (Schwarz et al. 2001). Thus further analysis of the active site of both enzymes by mutagenesis experiments is needed to explain their reaction mechanisms, since structural modelling is not possible due to the low sequence similarity to the available structures of soybean LOX-1 (Boyington et al. 1993) and rabbit reticulocyte 15-LOX (Gillmor et al. 1997).

\section{Cyanobacterial LOXs as putative ancestors of plant LOXs localised in the plastids}

In flowering plants 13-LOXs are not found ubiquitously in the cytosol and, until now, 9LOXs were only found within this compartment (Feussner and Wasternack 2002). Thus, the cytosol might be primarily the location of $(9 S)$-hydroperoxide-derived compounds. In contrast, in the plastid, only 13-LOXs have been detected so far. Therefore, (13S)- 
hydroperoxy PUFAs forming as well as metabolizing activities should be ubiquitously localized in plastids. Taken into consideration that cyanobacteria may be the ancestors of plastids the identification of NpLOX1 and 2 as 13-LOXs is in good agreement with this model. Even since the genome of $N$. punctiforme strain PCC73102 only seems to harbour these two 13-LOX genes. In addition the analysis of endogenous oxylipins in this cyanobacterium (Fig. 22) may indicate that this organism is only capable to form oxylipins that derive directly from the action of 13-LOXs. Additional enzymes metabolizing 13LOX-derived FA hydroperoxides known from the so-called LOX pathway in flowering plants are missing in $N$. punctiforme. Taken this into account it is tempting to assume that metabolic pathways involved in formation of more complex oxylipins like for instance the jasmonates or green leaf volatiles may have evolved later during evolution (Lang and Feussner 2007).

\subsection{The peroxidase-LOX fusion protein from Nostoc sp. SAG 25.82}

In addition to the LOXs from $N$. punctiforme, a naturally occurring peroxidaselipoxygenase fusion protein from the cyanobacterium Nostoc sp. SAG 25.82 was isolated and analysed. Amino acid sequence comparison revealed the highest homology with $34 \%$ to the AOS-LOX fusion protein from the coral Plexaura homomalla (Koljak et al. 1997). This coral enzyme converts the FA to the corresponding FA hydroperoxide, which is then further metabolized to an unstable allene oxide which immediately hydrolyzes to an $\alpha$ ketol (Koljak et al. 1997). However, despite of the similar protein domain structure any FA-derived ketols were detected as products from NspFP, and formation of FA dihydroxides was observed instead, when the protein was expressed in E. coli (III-3.3).

To gain more information on the biochemical features of NspFP the protein sequences of the separate domains were aligned with other characterized LOXs and peroxidase-like enzymes from plant, moss, coral and bacteria.

\section{Comparison of NspLOX domain with other LOXs}

An alignment of the deduced amino acid sequences of the peroxidase-domain with other peroxidase-like sequences from $O$. sativa, the coral $P$. homomalla and the bacterium Sulfitobacter sp. EE-36 showed that all sequences share conserved amino acids implicated in forming the active site of a peroxidase, either in heme binding or as catalytic residues (Fig. 25A, marked by stars). In the AOS domain of the coral fusion protein, three residues 
are important for coordinating the distal face of the heme group: Thr-66, His-67 and Asn137; the heme's proximal-side residues are occupied by Arg 349 and Tyr 353 (Koljak et al. 1997; Oldham et al. 2005). In the peroxidase domain of NspFP the residues coordinating the distal face of the heme group are conserved as Thr-40, His-41 and Asn-118. In contrast, the residues on the proximal face of the heme group differ from those of the coral enzyme, and instead of Arg-349 a glycine is situated at the corresponding position (Gly-323), while the position of Tyr-353 corresponds to His-327 with the neighbouring amino acid a tyrosine (Tyr-328).

The alignment of the deduced amino acid sequence of the NspFP LOX-domain with known LOX sequences shows, that the residues involved in iron binding within the active site are highly conserved and identical to those from plant LOXs: His-170, His-175, His342 and Asn-346. Although the fifth ligand did not align properly to the other sequences, it is represented by an isoleucine (Ile-429) as for other plant LOXs (Fig. 25B, li). At the position of the three determinants that may be involved in regiospecificity, NspFP contains different amino acids in comparison to the other LOX sequences from flowering plants (Sloane et al. 1995; Borngräber et al. 1996b; Hornung et al. 1999a): At the site described first by Sloane, a serine (Ser-229) is found instead of a phenylalanine in both plant LOXs (LOX2 At2 and PpLOX1; Fig. 25B, sl). These amino acids differ remarkably from the reported determinants for positional specificity of plant LOXs (Hornung et al. 1999a), precluding accurate prediction of positional specificity of the LOX-domain. In addition, an Arg residue reported to determine inverse substrate orientation in plant LOXs is missing as well in NspFP (Fig. 25B, ho) (Hornung et al. 1999a). However, the amino acid residue determining the stereospecificity of LOXs (Coffa site, Fig. 25B, cof) is an alanine residue, suggesting that this enzyme is an $S$-specific LOX.

Diagnostic differences or similarities within the structure of a LOX are mainly found around the active site residues of the protein (Liavonchanka and Feussner 2006). The iron binding site of LOXs is formed by an central histidine-rich region (Siedow 1991). Three highly conserved histidines were found in all four sequences (His-170, His-175, His-342). In addition two other conserved amino acids are involved in iron binding: an asparagine (Asn-346) and an isoleucine (Ile-429). Further on, the determinants responsible for the positional specificity of a LOX were analysed (Sloane et al. 1995; Borngräber et al. 1996b; Hornung et al. 1999a). At the position of the Borngräber site the LOX domain harbours a phenylalanine (Phe-162), which is rather bulky in comparison to serine (Ser-162) in 
Plexaura or alanine (Ala-162) in AtLOX3. At the position of the Sloane site the NspLOX domain harbours an alanine (Ala-227) and serine (Ser-228), whereas Plexaura has a serine (Ser-227) and leucine (Leu-228) at this position. Relying on the model explaining the positional specificity of plant LOXs (Hornung et al. 1999b), it was impossible to predict the positional specificity of the LOX domain in N. sp SAG 25.82. However, the space related model (Fig. 40) used for mammalian LOXs may be applicable to explain the positional specificity of the NspLOX domain.

Interestingly, the amino acid residue determining the stereospecificity of LOXs (Coffa site) was similar to that of other $S$-specific LOXs with an alanine (Ala-213) and not to that described for $R$-specific LOXs (Coffa and Brash 2004). The (8R)-LOX of the fusion protein from Plexaura harbours a glycine (Gly-213) at that site, but although NspFP harbours an alanine at the Coffa site the LOX domain was $(R)$-specific. Therefore it seems that more than one amino acid residue in the active site may be involved in the stereospecificity of the LOXs, at least for the LOX domain from N. sp. SAG 25.82. Thus, further mutagenesis experiments concerning the stereospecificity of LOXs will be necessary to explain the catalytic mechanism of this protein.

\section{Comparison of the N-terminal domain of NspFP with the AOS domain of the AOS-}

\section{LOX fusion protein from $P$. homomalla}

Besides the LOX-domain, the NspFP protein contained another protein module with similarity to peroxidase enzymes. The amino-terminal peroxidase domain of the coral fusion protein was described to catalyze an AOS-type reaction by metabolizing FA hydroperoxides into unstable allene oxides (Koljak et al. 1997). Although the enzyme domain of the coral enzyme catalyses the same reaction as plant AOS (Stumpe and Feussner 2006), the sequence similarity between plant AOS enzymes, which belong to the P450 superfamily, and the coral fusion-protein peroxidase domain is with about $6 \%$ identity rather low. A substantially higher similarity is apparent between the coral fusion protein peroxidase domain and catalases, and the solved crystal structure of the peroxidase domain of the coral protein showed a high structural similarity to other catalases. Catalases are specialized peroxidases that metabolize hydrogen peroxide. The protein harbours the catalase core" necessary for catalysis, however other parts of the catalase structure important i.e. for tetramerisation are missing (Oldham et al. 2005). Whereas the 
environment of the heme and the heme protein hydrogen-bonding network are highly conserved within the peroxidase domain of the coral fusion protein, there were differences to classical catalases in the planarity of the heme and the hydrogen bonding network at the proximal face of the heme. They may be responsible for the unusual reaction type and may convert the active site from a hydrogen peroxide transporting channel into a substrate binding pocket that is specific for FAs (Oldham et al. 2005). Indeed by exchanging Thr-66 into Val-66, the residue that is involved in the unique hydrogen bonding pattern of the coral fusion protein peroxidase domain, the AOS-activity of this domain was converted into a catalase (Tosha et al. 2006). The Thr-66 of the coral protein has an equivalent amino acid (Thr-42) in the peroxidase domain of NspFP, which may explain its substrate specificity for FA hydroperoxides. In the peroxidase domain of NspFP a number of highly conserved residues at the distal face of the heme group can also be found (Thr-40, His-41 and Asn-118). Interestingly the proximal Arg-349 of the coral enzyme has a much smaller pendant in NspFP (Gly-323), and Tyr-353 corresponds to His-327. The smaller glycine residue in NspFP may explain the catalytic differences between the coral enzyme and NspFP (as discussed below).

But structural modelling of the peroxidase domain of NspFP with the help of the structure of the coral AOS domain failed, which complicates the clarification of the reaction mechanism of NspFP.

\section{Reaction mechanism of NspFP}

In general the data obtained with recombinant NspFP lead to the hypothesis, that product formation underlies a general mechanism (Fig. 41), which can be briefly described as follows: The main products of the conversion of LA, ALA, GLA and AA have in common that the first hydroxyl group is formed at the carbon atom at position $n-10$. The insertion of the second hydroxyl group seems to be dependent on the double bond system of the substrate. Here the oxygen was either inserted at position $n-5$, if the substrate was a $\omega 6$ PUFA or at position n-3 if the substrate was a 133 -PUFA. This observation indicates that the fusion protein counts" from the methyl end of the substrate to insert the first oxygen molecule. 
9,14-diHODE

LA

9,16-diHOTE

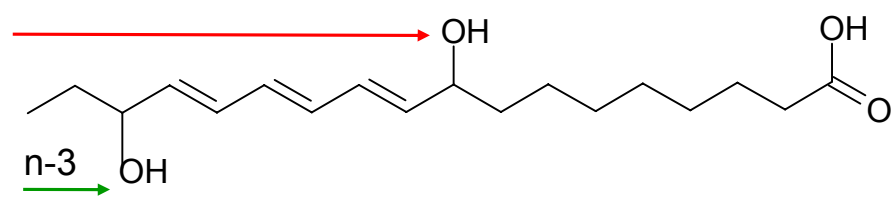

ALA

9,14-diHOTE

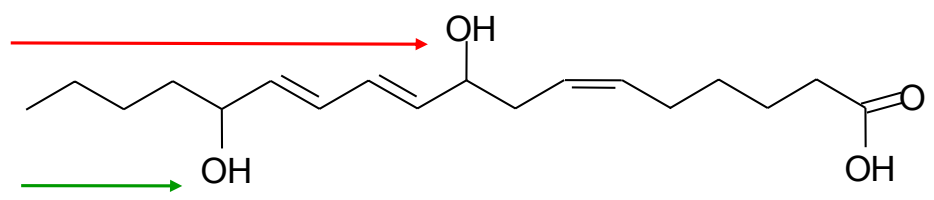

GLA

11,16-diHETE

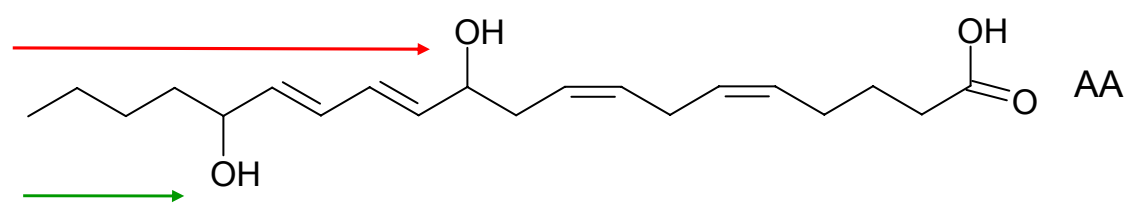

Figure 41. General scheme of NspFP reaction with LA, ALA, GLA and AA as substrates. The product formation seems to be related to the orientation of the substrate in the active site, since the enzyme always counts from the methyl end when the first oxygen is introduced.

The high sequence homology of the AOS-LOX fusion protein from $P$. homomalla and NspFP lead to the assumption, that both enzymes may share a similar reaction mechanism. But as described before the products of the reactions were different. Instead of ketols, which are the products of the AOS reaction in P. homomalla, NspFP forms dihydroxy FAs. The time course experiments performed (III-3.3.4) support the hypothesis that no unstable allene oxides are formed and released from the enzyme

However, the enzymatic formation of dihydroxy FAs was analysed and published before. In the marine macrophytic red algae Farlowia mollis and Gracilariopsis lemaneiformis two different dihydroxy FAs were isolated: (12R,13S)-dihydroxy-5Z,8Z,10E,14Zeicosatetraenoic acid $((12 R, 13 S)$-diHETE) and (12R,13S)-dihydroxy-5Z,8Z,10E,14Z,17Zeicosapentaenoic acid ((12R,13S)-diHEPE, Solem et al. 1989, Jiang \& Gerwick 1990). The reaction mechanism of the formation of $(12 R-13 S)$-diHETE was analysed with cell free preparations of $G$. lemaneiformis (Moghaddam and Gerwick 1991). Here the authors 
proposed three potential biosynthetic pathways with a initial 12-lipoxygenation of AA to $(5 Z, 8 Z, 10 E, 12 S, 14 Z)$-hydroperoxy-(5,8,10,14)-eicosatetraenoic acid ((12S)-HETE): (1) a trans- $(12 R, 13 R)$-epoxide formation and attack by $\mathrm{H}_{2} \mathrm{O}$ with inversion at $\mathrm{C}-13$, (2) a interor intramolecular rearrangement of the distal hydroperoxy oxygen of 12-HPETE or (3) an independent molecular oxygen oxidation of $\mathrm{C}-13$ by a separate catalytic activity. The origin of the oxygen molecules was confirmed by analysing the formation of $(12 R, 13 S)$ diHETE in presence of ${ }^{18} \mathrm{O}_{2}$. It was shown that both oxygen atoms at $\mathrm{C}-12$ and $\mathrm{C}-13$ derived from dioxygen, as the two hydroxyl groups were labelled with ${ }^{18} \mathrm{O}_{2}$ (Moghaddam and Gerwick 1991). Further on it was shown that two enzyme activities were involved in the conversion of AA into $(12 R, 13 S)$-diHETE. First a $(12 S)$-LOX reaction leads to the production of (12S)-HPETE, which is then further metabolised by a hydroperoxide isomerase, which catalyses the intramolecular rearrangement of (12S)-HPETE to the corresponding dihydroxy FA (Gerwick et al. 1991, Hamberg et al. 1993). Recently, Jiang and Gerwick (1997) described the production of oxylipins derived from a (9S)-LOX initiated pathway in the red alga Polyneura latissima. They proposed that the dihydroxy FA (5Z,7E,11Z,14Z)-9,15-dihydroxy-(5,7,11,14)-eicosatetraenoic acid (9,15-diHETE) was synthesised by further 15-lipoxygenation and reduction of (9S)-HPETE or (9S)-HETE.

Besides the discovery of dihydroxy FAs in algae these compounds were also found in the fungus Gaeumannomyces graminis (Brodowsky and Hamberg M. 1992). In G. graminis the oxygenation of LA is catalysed by a $(8 R)$-dioxygenase $((8 R)$-DOX) forming $(8 R)$ HPODE. This DOX differs from LOXs in their mechanism of oxygenation. In LOXs hydrogen abstraction and oxygen insertion happens at two different $\mathrm{C}$-atoms, due to radical rearrangement, whereas these two reactions are catalysed at the same carbon atom in by this DOX (Brodowsky et al. 1992). The resulting FA hydroperoxide can then either be reduced to $(8 R)$-HODE or metabolised to $(7 S, 8 S, 9 Z, 11 Z)$-dihydroxy- $(9,11)$ octadecadienoic acid $((7 S, 8 S)$-diHODE), which is catalysed by a hydroperoxide isomerase. Again, ${ }^{18} \mathrm{O}_{2}$ experiments were performed, to show, that the dihydroxy groups were both formed by intramolecular oxygenation of the FA hydroperoxide (Brodowsky and Hamberg M. 1992). Many efforts have been made to analyse the enzymes responsible for the sequential oxygenation in G. graminis and finally an enzyme named linoleate diol synthase (LDS) was isolated and further characterised as recombinant protein ( $\mathrm{Su}$ and Oliw 1996; Su 1998; Hörnsten et al. 1999; Oliw et al. 1999). It was shown, that LDS is a ferric hemoprotein, having a similar oxygenation mechanism as prostaglandin $\mathrm{H}$ ( $\mathrm{PGH})$ synthases, which occur in vertebrates but not in plants and lower eukaryotes (Hörnsten et 
al. 1999). The PGH synthase has two active sites: a cyclooxygenase site, responsible for the double dioxygenation of AA to hydroperoxy endoperoxide prostaglandin $\mathrm{H}_{2}\left(\mathrm{PGG}_{2}\right)$ and a heme with peroxidase activity, which is responsible for the reduction of $\mathrm{PGG}_{2}$ to prostaglandin $\mathrm{H}_{2}\left(\mathrm{PGH}_{2}\right)$ (Smith and Marnett 1991; Hla and Neilson 1992). During the cyclooxygenase reaction a tyrosyl radical and ferryl oxygen intermediates are formed, which were also found for the LDS. In addition, these two enzymes share significant amino acid similarities, suggesting that they belong to the same gene family of FA dioxygenases (Hörnsten et al. 2002).

In contrast to data published on the LOX/hydroperoxide isomerase reaction from $G$. lemaneiformis and the LDS from G. graminis, the formation of dihydroxy FAs by NspFP was different. Here the oxygen derived from $\mathrm{O}_{2}$ whereas the second oxygen came from water (III-3.3.4). Thus, unlike LDS and hydroperoxide isomerase, NspFP metabolises oxygen that derived from two different oxygen sources: Whereas the amino-terminal LOXdomain uses molecular oxygen, the carboxy-terminal peroxidase domain takes the oxygen atom from a water molecule to finish the reaction (Fig. 42), while the second oxygen from the FA hydroperoxide may be retained at the iron-centre of the heme group as has been proposed before (Tosha et al., 2006). Searching for structural differences possibly underlying the different catalytic activities of PhAOS-LOX and NspFP, the only obvious difference was the exchange of the proximal Arg-349 of the coral enzyme into a much smaller Gly-323 in their peroxidase domains. The proximal Arg residue is interacting with a Tyr, and it has been proposed to be important for "tuning" the heme so that oxidation occurs on the porphyrin ring (Oldham et al. 2005). This oxidation reaction seems to be altered in NspFP, since the detection of allene oxide formation failed and dihydroxy FAs were formed instead (Fig. 42, III-3.3.4). 


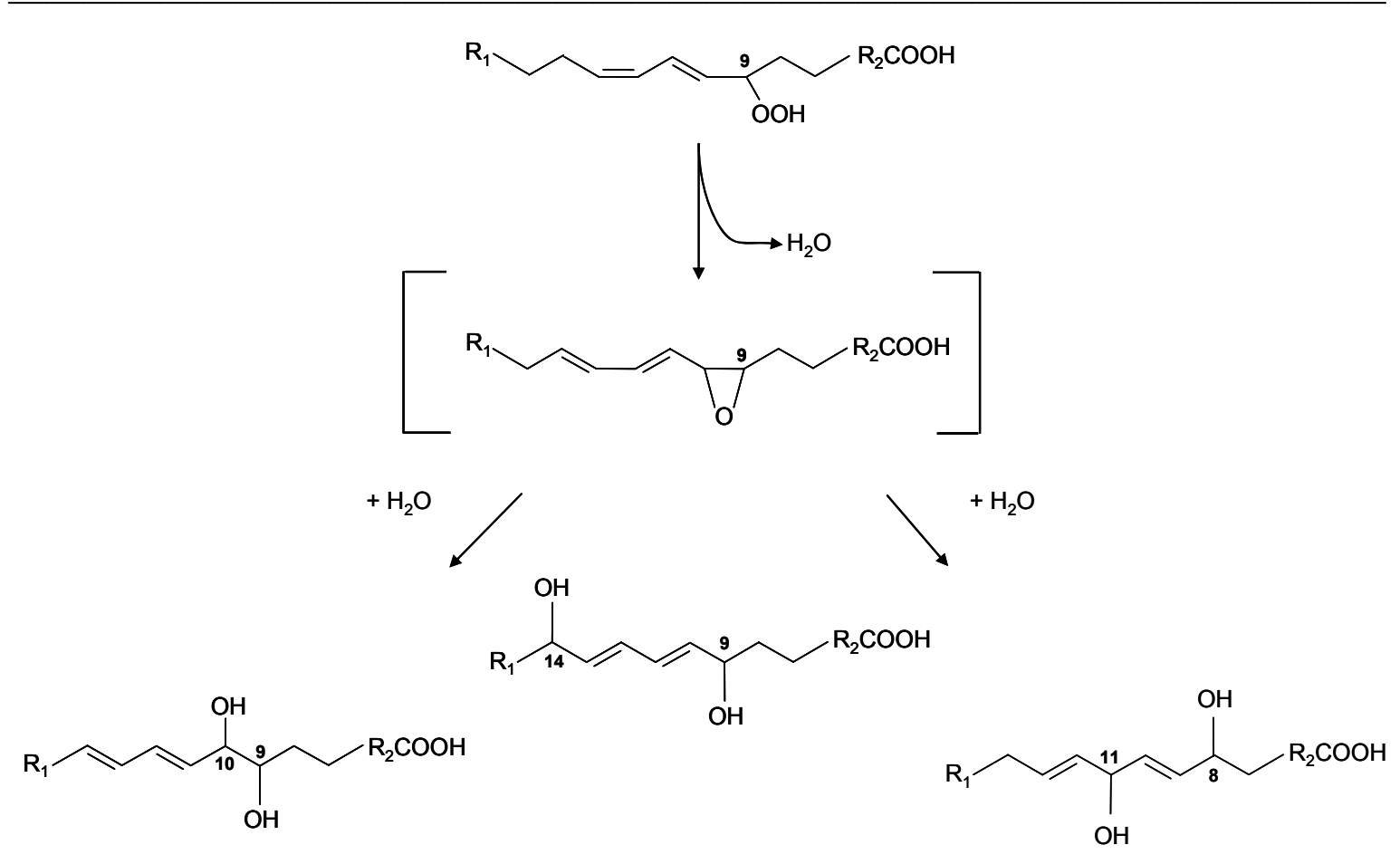

Figure 42 Mechanism proposed for the peroxidase domain of NspFP. Scheme of sequential oxygenation of LA by NspFP. NspFP has two enzymatic activities, linoleate $(9 R)$-LOX and peroxidase activity. LOX generates $(9 R)$-HPODE. The peroxidase activity reduces the lipid hydroperoxide to yield an epoxide and rearrangement of conjugated double bonds. The reaction is finished by addition of water to this intermediate.

\section{Biological role of NspFP}

The analysis of the recombinant NspFP raised the question about the function of the dihydroxy FAs in Nostoc sp. in vivo. It is known that LOXs get activated and hydroperoxides as well as cleavage products are present when the integrity of the cells in algae is destroyed (Jüttner 1995). Hence, one objective of this work was to identify products that may derive from the activity of NspFP in vivo.

Therefore the analysis of endogenous oxylipins detected in Nostoc sp. PCC7120 may give first indications for potential physiological functions of enzymes of the LOX pathway in cyanobacteria. Analysis of oxylipin profiles in Nostoc sp. PCC7120 indicates that this organism is only capable to form oxylipins that derive directly from the action of the LOX domain of NspFP, at least under the used conditions. Surprisingly, neither products that derived from the activity of the peroxidase domain nor that of additional enzymes metabolizing the 9-LOX-derived FA hydroperoxides known from the so-called LOX pathway in flowering plants were found and, thus, such enzymes seem to be absent. This 
notion is further supported by results from the more sensitive radio labelling experiments. The observation that the peroxidase domain was not active in vivo may be explained by the presence of an inhibitory cofactor under our experimental conditions in whole cells or extracts that was missing when NspFP was expressed in E. coli. Besides the gene encoding NspFP, no other open reading frames were found in the genome of Nostoc sp PCC7120 that may encode additional LOXs or other enzymes of the LOX pathway as it is known from flowering plants. However, it should be noted that the appearance of $(9 R)$ HOTE/HODE is not unusual in cyanobacteria of the genus Anabaena, since it has been reported before (Murakami et al. 1992). Moreover, (9R)-HOTE/HODE has been shown to occur in the eukaryotic alga, Ulva conglobata (Akakabe et al. 2002), and dihydroxy FAs similar in structure to those formed in vitro by NspFP have been described to occur in red algae (Hamberg and Gerwick 1993).

As discussed before, (13S)-hydroperoxy PUFAs forming as well as metabolizing activities should be ubiquitously localized in plastids. Taken into consideration that cyanobacteria may be the ancestors of plastids (ref.) the identification of the NpLOX1 and 2 enzymes from the cyanobacterium $N$. punctiforme (PCC73102) as 13-LOXs was in good agreement with this model (Koeduka et al. 2007; Lang and Feussner 2007). While the identification of NspFP as a (9R)-LOX appears to contradict this model, it may be explained by the fact that NspFP is localized on the Gamma plasmid of this bacterium and, thus, it is tempting to assume that it was taken up by horizontal gene transfer from other bacteria. The similar gene structure of PhAOS-LOX and NspFP and the fact, that many corals live in symbiosis with algae, so called zooxanthellea, may suggest a common algal origin of such enzymes with two catalytically active sites. But since only a restricted search in published genomes of bacteria and microalgae is possible, it is too early to answer this question. So far PhAOS-LOX and NspFP display the only enzymes with such gene structure known so far. In summary, it is tempting to speculate that metabolic pathways involved in the formation of more complex oxylipins may have evolved at a later point during evolution, whereas the use of bi- or multifunctional enzymes may represent a more ancient way to form oxylipins. Moreover, Nostoc sp. may harbour different defence mechanism respectively. For instance the cyanobacterium Lyngbya majuscula developed another chemical defence against a variety of consumers by producing the nitrogenous secondary metabolite lyngbyatoxin A (Capper et al. 2006). Thus Nostoc sp could have evolved a similar strategy to protect itself against wounding 
In conclusion, the results presented in this thesis indicate that a large scale profiling of lipid bound FAs is a valuable tool in order to find microalgal strains which produce VLCPUFAs in high proportions. These microalgae may be suitable for VLCPUFA production in chemical industry, human nutrition or as gene sources for plant engineering. Furthermore the results indicate that there is a causative relation between the endosymbiosis theory of microalgal evolution and FA composition of microalgae. The use of certain FAs, like 18:5n-3, as biomarkers may be possible, if the FA is present in significant proportions and restricted to one or two closely related algal groups. Growth conditions and age of the culture influence the FA composition of microalgae, accounting for differences of FA profiles between data obtained in this thesis and data available from the literature.

The analyses of lipid and FA composition of P. parvum in the presence and absence of labelled FAs indicate that this approach is useful to unravel the biosynthetic pathway of 18:5n-3. The results obtained indicate the existence of a so far unknown $\Delta 3$-desaturase, which may introduce a double bond at position 3 into 18:4n-3 resulting in the formation of 18:5n-3.

The isolation and characterisation of two linoleate $13 S$-LOXs from $N$. punctiforme and the analysis of endogenous oxylipins support the hypothesis that cyanobacteria may be the ancestors of plastids, since only 13-LOXs have been detected in this organelle so far. Amino acid sequence comparisons of LOXs showed, that the determinants involved in the regio- and stereospecificity in $N$. punctiforme LOXs differ from those of known plant and mammalian LOXs. The peroxidase-LOX fusion protein from the cyanobacterium Nostoc sp. termed NspFP and identified in the present study, is a candidate for a cyanobacterial enzyme generating LOX products. NspFP has a putative function in stress adaptation of cyanobacteria to wounding, since LOX derived products increased after sonification of the cultures. Recombinant NspFP converts C18 and C20 PUFAs into the corresponding dihydroxy FAs, whereby the product formation seems to underlie a general mechanism. The LOX domain uses molecular oxygen to introduce the first hydroxyl group at position $\mathrm{n}-10$, whereas the carboxy-terminal peroxidase domain takes the oxygen atom from a water molecule to insert oxygen either at position $n-5$, if the substrate is a $\omega 6$ PUFA, or at position $n-3$ if the substrate is a $\omega 3$-PUFA. 


\section{Outlook}

So far only the most eminent results of the database have been looked at and the entire information content still has to be extracted. Since there a still some FAs, for which the double bond positions have not been determined yet, the first step shall be their identification by DMOX or picolinyl derivatives, respectively.

In general it is aimed to link the database presented in this thesis to the microalgal database of the SAG. Amongst other databases the SAG database already provides information about growth conditions, the original habitat from which the strains have been isolated, current state of the culture and notes on the use of some strains for industrial applications. The addition of the FA profiles of each strain will enhance this database, since it will facilitate the choice of a microalgal strain for further analysis regarding FA metabolism. Thus, microalgae harbouring interesting PUFAs, which may be precursors for unknown oxylipins, may be used for oxylipin profiling, to identify valuable substances in food and flavour industry.

The database may further be used to elucidate chemotaxonomy among microalgae, by comparing the phylogenetic relationship of microalgae based on 16SrDNA and 18SrDNA sequence analyses and their FA profiles obtained. As mentioned before several parameters can be selected to discriminate taxonomic groups with respect to their FA content. Furthermore the overall FA profile among of genera or classes and the proportion of carbon atoms and double bonds can be studied. Initial taxonomic analyses using this database should be carried out on algal strains which have been already described in other studies in this respect to verify the quality of the data obtained in this work.

Microalgal strains containing high amounts of PUFAs and VLCPUFAs may further be tested for their potential for industrial production of the respective FAs. This goal may be reached by varying the growth conditions of the respective algal cultures and analysing the resulting lipid and FA profiles.

Furthermore microalgae suitable for the isolation of enzymes involved in the biosynthesis of VLCPUFA may be analysed in more detail. In particular, the FA metabolism of $P$. varium may be studied, by analysing the lipid and FA composition in presence and absence of labelled FAs. According to other studies labelled $\left[1-{ }^{14} \mathrm{C}\right]$-acetate may be used to confirm the existence of a PKS related pathway in P. varium (Metz et al 2001). The absence of labelled precursor products of AA may support this hypothesis.

Based on the assumption that 18:5n-3 may be synthesised via a $\Delta 3$-desaturase, which introduces a double bond at position 3 into 18:4n-3, one future project may be the isolation 
of the corresponding gene and the characterisation of the enzyme. Moreover 18:5n-3 is discussed to constitute an alternative supplement for EPA in human diet. In order to produce 18:5n-3 in flowering plants, the enzymes involved in 18:5n-3 biosynthesis have to be transferred into the plants. In parallel the metabolism of 18:5n-3 supplemented to mammalian cells can be investigated, since it is not clear if 18:5n-3 is elongated to EPA by a $\Delta 3$-specific elongase or if its metabolism is similar to that in fish cells (Ghioni et al 2001).

The two (13S)-LOXs isolated from $N$. punctiforme may constitute interesting enzymes to study the stereo- and regiospecificity of active site amino acid residues, since the active site is neither similar to mammalian LOXs nor to plant LOXs. The purification and crystallisation of the LOXs in combination with mutagenesis experiments may be a helpful approach to explain the reaction mechanisms. Moreover, both LOXs exclusively produce $13 S$-hydroperoxides around neutral $\mathrm{pH}$, which may be of importance for oxylipin production in biotechnology.

In order to elucidate the reaction mechanism of the peroxidase-LOX fusion protein, it may be useful to purify the protein and to obtain a crystal structure. The co-crystallisation of the enzyme with the FA substrate as well as with the intermediate hydroperoxy FAs may give an idea, how the two domains interact. Site directed mutagenesis of amino acid residues in the active site of the enzyme may help to identify determinants involved in dihydroxy FA formation. 


\section{Summary}

In the present thesis a large scale profiling of lipid bound FAs (C14-C24) was performed with all SAG algal strains available. In total 2347 microalgal strains have been analysed and 87 different substances were detected, whereby 77 represent methyl ethers of FAs. A database has been established using the Microsoft Excel format, which contains all identified FAMEs and some other metabolites. The database provides information about the FAME content of each microalga strain, which can further be used for the identification of microalgae producing PUFAs and VLCPUFAs in high amounts. An initial comparison of FA profiles of different microalgal strains showed that FA profiles were similar among the same species but different among genera and classes. The PUFA 18:5n3 was found to be specific for the marine algal classes, Dinophyceae, Haptophyceae and Raphidophyceae, whereas the PUFA pinolenic acid was found to be specific for the green algae Chlamydomonas and Chlamydocapsa.

The biosynthetic pathway of 18:5n-3 was investigated by analysing the fate of exogenously fed labelled FAs in P. parvum. The supplementation of labelled LA and ALA lead to the synthesis of labelled 18:4n-3, 18:5n-3 and EPA, whereas the supplementation of labelled EPA lead to the synthesis of labelled 22:5n-3 and 22:6n-3, indicating that 18:5n-3 may be synthesised via a $\Delta 3$-desaturase which introduces a double bond into 18:4n-3.

In addition to the FAME screening one further aim of this thesis was the identification of oxylipin producing microalgae. Since the initial oxylipin screening of cyanobacteria was not successful, enzymes involved in oxylipin biosynthesis were isolated. Two linoleate (13S)-LOXs from the cyanobacterium N. punctiforme PCC 73102 were isolated and characterised. The determinants involved in the regiospecificity of both enzymes were neither similar to mammalian LOXs nor to plant LOXs and so far the reaction mechanism cannot be explained with the present data. The analysis of endogenous oxylipins in $N$. punctiforme showed, that only 13-LOX derived FA hydroperoxides were formed, whereas the LOX activity increased after treating the cells with sonification. In addition a naturally occurring peroxidase-LOX fusion protein from the cyanobacterium Nostoc sp. SAG 25.82 was isolated and characterised. The C-terminal LOX domain was identified as a linoleate $(9 R)$-LOX and the N-terminal peroxidase domain converted the LOX derived hydroperoxides to the corresponding dihydroxides. The analysis of endogenous oxylipins in $N$. $s p$. showed that only 9-LOX derived FA hydroperoxides were formed, but no products derived from the peroxidase activity were detected. 


\section{Literature Cited}

Abbadi, A., F. Domergue, J. Bauer, J. A. Napier, R. Welti, U. Zähringer, P. Cirpus and E. Heinz (2004). Biosynthesis of very-long-chain polyunsaturated fatty acids in transgenic oilseeds: constraints on their accumulation. The Plant Cell. 16(10): 2734-48

Adolph, S., S. Bach, M. Blondel, A. Cueff, M. Moreau, G. Pohnert, S. A. Poulet, T. Wichard and A. Zuccaro (2004). Cytotoxicity of diatom-derived oxylipins in organisms belonging to different phyla. Journal of Experimental Biology 207(Pt 17): 2935-46.

Akakabe, Y., M. K. and K. T. (2002). Enantioselective formation of (R)-9-HPODE and $(R)-9-H P O T r E$ in marine green alga Ulva conglobata. Bioorganic \& Medical Chemistry 10: $3171-3173$.

Alonso, D. L., E.-H. Belarbim, J. Rodriguez-Ruiz, C. I. Segura and A. Giménez (1998). Acyl lipids of three microalgae. Phytochemistry 47(8): 1473-1481.

Apt, K. E. and P. W. Behrens (1999). Commercial developments in microalgal biotechnology. Journal of Phycology 35: 215-226.

Armbrust, E. V., J. A. Berges, C. Bowler, B. R. Green, D. Martinez, N. H. Putnam, S. Zhou, A. E. Allen, K. E. Apt, M. Bechner, M. A. Brzezinski, B. K. Chaal, A. Chiovitti, A. K. Davis, M. S. Demarest, J. C. Detter, T. Glavina, D. Goodstein, M. Z. Hadi, U. Hellsten, M. Hildebrand, B. D. Jenkins, J. Jurka, V. V. Kapitonov, N. Kroger, W. W. Lau, T. W. Lane, F. W. Larimer, J. C. Lippmeier, S. Lucas, M. Medina, A. Montsant, M. Obornik, M. S. Parker, B. Palenik, G. J. Pazour, P. M. Richardson, T. A. Rynearson, M. A. Saito, D. C. Schwartz, K. Thamatrakoln, K. Valentin, A. Vardi, F. P. Wilkerson and D. S. Rokhsar (2004). The genome of the diatom Thalassiosira pseudonana: ecology, evolution, and metabolism. Science 306(5693): 79-86.

Arzul, G., P. Gentien, G. Bodennec, F. Toularastel, A. Youenou and M. P. Crassous (1995). Comparison of toxic effects in Gymnodinium ef nagasakiense polyunsaturated fatty acids. Harmful Marine Algal Blooms. P. Lassus, E. Arzul, P. Erardet al. Paris, Lavoisier, Intercept Ltd: 395-400.

Athenstaedt, K. and G. Daum (2006). The life cycle of neutral lipids: synthesis, storage and degradation. Cell Mol Life Sci 63(12): 1355-69.

Ausubel, F. M., R. Brent, R. E. Kingston, D. D. Moore, J. G. Seidman, J. A. Smith and K. Struhl (1993). Current protocols in molecular biology, John Wiley \& Sons Inc.

Bachvaroff, T. R., G. T. Concepcion, C. R. Rogers, E. M. Herman and C. F. Delwiche (2004). Dinoflagellate expressed sequence tag data indicate massive transfer of chloroplast genes to the nuclear genome. Protist 155(1): 65-78.

Batthacharya, D. and L. Medlin (2005). The phylogeny of plastids: a review based on comparison of small-subunit ribosomal RNA coding regions. Journal of Phycology 31: 489-498. 
Bell, V. M., J. R. Dick and D. W. Pond (1997). Octadecapentaenoic acid in a raphidophyte alga, Heterosigma akashiwo. Phytochemistry 2: 303-306.

Beneytout, J.-L., R.-H. Andrianarison, Z. Rakotoarisoa and M. Tixier (1989). Properties of a lipoxygenase in blue-green algae (Oscillatoria sp.). Plant Physiology 91: 367-372.

Benning, C., Z. H. Huang and D. A. Gage (1995). Accumulation of a novel glycolipid and a betaine lipid in cells of Rhodobacter sphaeroides grown under phosphate limitation. Arch Biochem Biophys 317(1): 103-11.

Bentley, R. and J. W. Bennett (1999). Constructing polyketides: from collie to combinatorial biosynthesis. Annu Rev Microbiol 53: 411-46.

Bergé, J.-P. and G. Barnathan (2005). Fatty acids from lipids of marine organism: molecular biodiversity, roles as biomarkers, biologically active compounds and economical aspects. Adv Biochem Engin 96: 49-125.

Bernart, M. W., G. G. Whatley and W. H. Gerwick (1993). Unprecedented oxylipins from the marine green alga Acrosiphonia coalita. J Nat Prod 56(2): 245-59.

Bertani, G. (1951). Studies on lysogenesis. I. The mode of phage liberation by lysogenic Escherichia coli. J Bacteriol 62(3): 293-300.

Bigogno, C., I. Khozin-Goldberg, S. Boussiba, A. Vonshak and Z. Cohen (2002). Lipid and fatty acid composition of the green oleaginous alga Parietochloris incisa, the richest plant source of arachidonic acid. Phytochemistry 60(5): 497-503.

Birnboim, H. C. and J. Doly (1979). A rapid alkaline extraction procedure for screening recombinant plasmid DNA. Nucleic Acids Res 7(6): 1513-23.

Bleé, E. (1998). Phytooxylipins and plant defense reactions. Prig. Lipids Res. 37(1): 3372 .

Bleé, E. (2002). Impact of phyto-oxylipins in plant defense. Trends in Plant Science 7(7): 315-321.

Bleé, E. and J. Joyard (1996). Envelope membranes from spinach chloroplasts are a site of metabolism of fatty acid hydroperoxides. Plant Physiology 110: 445-454.

Bligh, E. G. and W. J. Dyer (1959). A rapid method of total lipid extraction and purification. Canadian Journal of Biochemistry and Physiology 37: 911-917.

Blunt, J. W., B. R. Copp, M. H. G. Munro, P. T. Northcote and M. R. Prinsep (2005). Marine natural products 22: 15-61.

Borngräber, S., R. J. Kuban, M. Anton and H. Kühn (1996a). Phenylalanine 353 is a primary determinant for the positional specificity of mammalian 15-lipoxygenases. Journal of Molecular Biology 264(5): 1145-1153. 
Borngräber, S., R. J. Kuban, M. Anton and H. Kühn (1996b). Phenylalanine 353 is primary determinant for the positional specificity of mammalian 15-lipoxygenases. Journal of Molecular Biology 264: 1145-1153.

Borowitzka, M. A. (1999). Commercial production of microalgae: ponds, tanks, tubes and fermenters. J. Biotechnol. 70: 313-321.

Boyington, J. C., B. J. Gaffney and L. M. Amzel (1993). Structure of soybean lipoxygenase-I. Biochem Soc Trans 21 (Pt 3)(3): 744-8.

Bradford, M. M. (1976). A Rapid and Sensitive Method for the Quantitation of Microgram Quantities of Protein Utilizing the Principle of Protein-Dye Binding. Anal. Biochem. 72: 248-254.

Brash, A. R. (1999). Lipoxygenases: occurrence, functions, catalysis and acquisition of substrate. Journal of Biological Chemistry 274(34): 23679-23682.

Brodowsky, I. D. and O. E. Hamberg M. (1992). A linoleic acid (8R)-dioxygenase and hydroperoxide isomerase of the fungus Gaeumannomyces graminis. The Journal of Biological Chemistry 267(21): 14738-14745.

Brown, M. R., M. Mular, I. Miller, C. Farmer and C. Trenerry (1999). The vitamin content of microalgae used in aquaculture. Journal of Applied Phycology 11: 247-255.

Browner, M. F., S. A. Gillmor and R. Fletterick (1998). Burying a charge. Nature Structural Biology 5(3): 179.

Browse, J. and C. R. Somerville (1994). Glycerolipids. Arabidopsis. M. a. Somerville. Cold Spring Harbour, NY, Cold Spring Harbour Laboratory Press: 881-912.

Browse, J., N. Warwick, C. R. Somerville and C. R. Slack (1986). Fluxes through the prokaryotic and eukaryotic pathways of lipid synthesis in the '16:3' plant Arabidopsis thaliana. Biochemical Journal 235: 25-31.

Bullock, W. O., J. M. Fernandez and J. M. Short (1989). A high efficiency plasmid transforming recA Escherichia coli strain with beta-galactosidase selection. BioTechniqes 5: 376-379.

Burja, A. M., B. Banaigs, E. Abou-Mansour, J. G. Burguess and P. C. Wright (2001). Marine cyanobacteria-a profilic source of natural products. Tetrahedron 57: 9347-9377.

Cannell, R. (1993). Algae as a source of biologically acitve products. Pesticide Science 39: $147-153$.

Capper, A., E. Cruz-Riviera, V. J. Paul and I. R. Tibbets (2006). Chemical deterrence of a marine cyanobacterium against sympatric and non-sympatric consumers. Hydrobiologia 553: 319-326.

Cardellina, J. H. and R. E. Moore (1980). Tetrahedron 36: 993-996. 
Cardozo, K. H., T. Guaratini, M. P. Barros, V. R. Falcao, A. P. Tonon, N. P. Lopes, S. Campos, M. A. Torres, A. O. Souza, P. Colepicolo and E. Pinto (2006). Metabolites from algae with economical impact. Comp Biochem Physiol C Toxicol Pharmacol.

Carman, G. M. (2005). Regulation of phospholipid synthesis in yeast by zinc. Biochemical Society Transactions 33(5): 1150-1153.

Caudales, R. and J. M. Wells (1992). Differentiation of the free-living Anabaena and Nostoc cyanobacteria on the basis of fatty acid composition. Int. J. Syst. Bacteriol. 42: 246-251.

Cavalier-Smith, T. (1986). The kingdoms of organisms. Nature 324(6096): 416-7.

Cavalier-Smith, T. (1999). The principles of protein and lipid targeting in secondary symbiogenesis: euglenoid, dinoflagellate and sporozoan plastid origins and the eukaryote family tree. J. Eukaryot. Microbiol. 46: 347-366.

Chini Zittelli, G., F. Lavista, A. Bastianini, L. Rodolfi, M. Vincenzini and M. R. Tredici (1999). Production of eicosapentaenoic acid by Nannochloropsis sp. cultures in outdoor tubular photobioreactors. 70: 299-312.

Choi, J.-Y., J. Stukey, S.-Y. Hwang and C. E. Martin (1996). Regulatory elements that control transcription activation and unsaturated fatty acid- mediated repression of the Saccharomyces cerevisiae OLE1 gene. The Journal of Biological Chemistry 271(7): 3581-3589.

Coffa, G. and A. R. Brash (2004). A single active site residue directs oxygenation stereospecificity in lipoxygenases: stereocontrol is linked to the position of oxygenation. Proc Natl Acad Sci U S A 101(44): 15579-15584.

Cohen, Z. (1990). The production potential of eicosapentaenoic and arachidonic acids by the red alga Porphyridium cruentum. J. Am. Oil Chem Soc. 67: 916-920.

Cohen, Z. (1999). Porphyridium cruentum. Chemicals from microalgae. Z. Cohen. London, Taylor \& Francis Ltd: 1-24.

Cohen, Z. and I. Khozin-Goldberg (2005). Searching for PUFA-rich microalgae. Single cell oils. Z. Cohen and C. Ratledge. Champaign, Illinois, AOCS Press: 53-72.

Cohen, Z., A. Vonshak and A. Richmond (1988). Effect of enviromental conditions on fatty acid composition of the red alga Porphyridium cruentum: correlation to growth rate. Journal of Phycology 24: 328-332.

Corpet, F. (1988). Multiple sequence alignment with hierarchical clustering. Nucleic Acids Res 16(22): 10881-90.

d'Ippolito, G., S. Tucci, A. Cutignano, G. Romano, G. Cimino, A. Miralto and A. Fontana (2004). The role of complex lipids in the synthesis of bioactive aldehydes of the marine diatom Skeletonema costatum. Biochimica et Biophysica Acta 1686: 100107. 
Dahlqvist, A., U. Stahl, M. Lenman, A. Banas, M. Lee, L. Sandager, H. Ronne and S. Stymne (2000). Phospholipid:diacylglycerol acyltransferase: an enzyme that catalyzes the acyl-CoA-independent formation of triacylglycerol in yeast and plants. Proc Natl Acad Sci U S A 97(12): 6487-92.

Damude, H. G. and A. J. Kinney (2007). Engineering oilseed plants for a sustainable, land-based source of long chain polyunsaturated fatty acids. Lipids 42: 179-185.

Dembitsky, V. M. (1996). Betaine ether-linked glycerolipids: chemistry and biology. Prig. Lipids Res. 35(1): 1-51.

Dembitsky, V. M. and M. Srebnik (2002). Variability of hydrocarbon and fatty acid components in cultures of the filamentous cyanobacterium Scytonema sp. isolated from the microbial community 'Black Cover' of limestone walls in Jerusalem. Biochemistry (Moscow), Eng. Ed. 67: 1545-1552.

Destaillats, F.; Angers, P. (2002) One-step methodology for the synthesis of fatty acid picolinyl esters from intact lipids. J. Am. Oil Chem. Soc. 79: 253-256

Di Venere, A., M. L. Salucci, G. van Zadelhoff, G. Veldink, G. Mei, N. Rosato, A. Finazzi-Agro and M. Maccarrone (2003). Structure-to-function relationship of minilipoxygenase, a $60-\mathrm{kDa}$ fragment of soybean lipoxygenase-1 with lower stability but higher enzymatic activity. J. Biol. Chem. 278(20): 18281-18288.

Domergue, F., A. Abbadi, U. Zähringer, H. Moreau and E. Heinz (2005). In vivo characterisation of the first acyl-CoA $\Delta^{6}$-desaturase from a member of the plant kingdom, the microalga Ostreococcus tauri. Biochemical Journal 389: 483-490.

Drexler, H., P. Spiekermann, A. Meyer, F. Domergue, T. Zank, P. Sperling, A. Abbadi and E. Heinz (2003). Metabolic engineering of fatty acids for breeding of new oilseed crops: strategies, problems and first results. Journal of Plant Physiology 160(7): 779802.

Dunstan, G. A., M. R. Brown and J. K. Volkman (2005). Cryptophyceae and Rhodophyceae; chemotaxonomy, phylogeny and application. Phytochemistry 66: 25572570 .

Dunstan, G. A., J. K. Volkman, S. M. Barnett, J.-M. Leroi and S. W. Jeffrey (1994). Essential polyunsaturated fatty acids from 14 species of diatom (Bacillariophyceae). Phytochemistry 35(1): 155-161.

Dunstan, G. A., J. K. Volkman, J. S.W. and S. M. Barrett (1992). Biochemical composition of microalgae from the green algal classes Chlorophyceae and Prasinophyceae. 2. Lipid classes and fatty acids. J. Exp. Mar. Biol. Ecol. 161: 115-134.

Eichenberger, W. (1976). Lipids of Chlamydomonas reinhardtii under different growth conditions. Phytochemistry 15: 459-463.

Eichenberger, W. (1993). Plant Physiol. Biochem. 31: 213-221. 
Eichenberger, W. and C. Gribi (1997). Lipids of Pavlova lutheri: cellular site and metabolic role of DGCC. Phytochemistry 45(8): 1561-1567.

Fay, L. and U. Richli (1991). Location of double bonds in polyunsaturated fatty acids by gas chromatography-mass spectrometry after 4,4-dimethyloxazoline derivatization. Journal of Chromatography A 541: 89-98.

Feussner, I. and H. Kühn (2000). Application of lipoxygenases and related enzymes for the preparation of oxygenated lipids. Enzymes in Lipid Modification. U. T. Bornscheuer. Weinheim, Germany, Wiley-VCH: 309-336.

Feussner, I. and S. Rosahl (2005). Oxylipins. Plant Lipids - Biology, Utilisation and Manipulation. D. Murphy, Blackwell publishing.

Feussner, I. and C. Wasternack (2002). The lipoxygenase pathway. Annu. Rev. Plant Biol. 53: 275-297.

Folch, J., M. Lees and G. H. S. Stanley (1957). A simple method for the isolation and purification of total lipides from animal tissues. J Biol Chem 266: 497-509.

Frentzen, M. (1993). Acyltransferases and triacylglycerols. Lipid Metabolism in Plants. T. S. Moore. Boca Raton, FL, CRC Press: 195-231.

Gardner, H. W. (1989). Soybean lipoxygenase-1 enzymatically forms both 9(S)- and 13(S)-hydroperoxides from linoleic acid by a $\mathrm{pH}$-dependent mechanism. Biochimica et Biophysica Acta 1001: 274-281.

Gerwick, W. H. (1994). Structure and biosynthesis of marine algal oxylipins. Biochimica et Biophysica Acta 1211: 243-255.

Gerwick, W. H. (1999a). Eicosanoids in nonmammals. Comprehensive Natural Products Chemistry: Polyketides and other secondary metabolites including fatty acids and their derivatives. D. Barton, K. Nakanishi and O. Meth-Cohn. Amsterdam, Elsevier. 1: 207-254.

Gerwick, W. H. (1999b). Eicosanoids in nonmammals. Comprehensive Natural Products Chemistry. U. Sankawa. Amsterdam, Lausanne, New York, Oxford, Shannon, Singapore, Tokyo, Elsevier. 1: 207-254.

Ghioni, C., A. E. A. Porter, I. H. Sadler, D. R. Tocher and J. R. Sargent (2001). Cultured fish cells metabolize octadecapentaenoic acid (all-cis $\Delta 3,6,9,12,15-18: 5$ ) to octadecatetraenoic acid (all-cis $\Delta 6,9,12,15-18: 4$ ) via its 2-trans intermediate (trans $\Delta 2$, all-cis $\Delta 6,9,12,15-18: 5)$. Lipids 36(2): 145-153.

Gill, I. and R. Valivety (1997). Polyunsaturated fatty acids, Part 1: Occurrence, biological activities and applications. Trends Biotechnol. 15(10): 401-409.

Gillmor, S. A., A. Villasenor, R. Fletterick, E. Sigal and M. F. Browner (1997). The structure of mammalian 15-lipoxygenase reveals similarity to the lipases and the determinants of substrate specificity. Nat Struct Biol 4(12): 1003-9. 
Gilson, P. R. (2001). Nucleomorph genomes: much ado about practically nothing. Genome Biol 2(8): REVIEWS1022.

Giroud, C. and W. Eichenberger (1989). Lipids of Chlamydomonas reinhardtii. Incorporation of $\left[{ }^{14} \mathrm{C}\right]$ acetate, $\left[{ }^{14} \mathrm{C}\right]$ palmitate and $\left[{ }^{14} \mathrm{C}\right]$ oleate into different lipids and evidence for lipid linked desaturation of fatty acids. Plant Cell Physiol. 30(1): 121-128.

Giroud, C., A. Gerber and W. Eichenberger (1988). Lipids of Chlamydomonas reinhardtii. Analysis of molecular species and intracellular site(s) of biosynthesis. Plant Cell Physiol. 29(4): 587-595.

Göbel, C., I. Feussner and S. Rosahl (2003). Lipid peroxidation during the hypersensitive response in potato in the absence of 9-lipoxygenases. J. Biol. Chem. 278(52): 52834-52840.

Gohil, V. M., P. Hayes, S. Matsuyama, H. Schagger, M. Schlame and M. L. Greenberg (2004). Cardiolipin biosynthesis and mitochondrial respiratory chain function are interdependent. Journal of Biological Chemistry 279(41): 42612-8.

Gray, M. W. (1992). The endosymbiont hypothesis revisited. Int. Rev. Cytol. 141: 233357.

Grechkin, A. N. (1998). Recent developments in biochemistry of the plant lipoxygenase pathway. Prog. Lipids Res. 37(5): 317-352.

Guschina, I. A. and J. L. Harwood (2006a). Lead and copper effects on lipid metabolism in cultured lichen photobionts with different phosphorus status. Phytochemistry 67(16): 1731-9.

Guschina, I. A. and J. L. Harwood (2006b). Lipids and lipid metabolism in eukaryotic algae. Prog Lipid Res 45(2): 160-86.

Hackett, J. D., T. E. Scheetz, H. S. Yoon, M. B. Soares, M. F. Bonaldo, T. L. Casavant and D. Bhattacharya (2005). Insights into a dinoflagellate genome through expressed sequence tag analysis. BMC Genomics 6(1): 80.

Haigh, W. G., T. F. Yoder, L. Ericson, T. Pratum and R. R. Winget (1996). The characterisation and cyclic production of a highly unsaturated homoserine lipid Chlorella minutissima. Biochimica et Biophysica Acta 1299: 183-190.

Halim, V. A., A. Vess, D. Scheel and S. Rosahl (2006). The role of salicylic acid and jasmonic acid in pathogen defence. Plant Biol (Stuttg) 8(3): 307-13.

Hamberg, M. (1988). Biosynthesis of 12-Oxo-10,15 (Z)-phytodienoic acid: identification of an allene oxide cyclase. Biochemical and Biophysical Research Communications 156(1): 543-550.

Hamberg, M. (1999). An epoxy alcohol synthase pathway in higher plants: biosynthesis of antifungal trihydroxy oxylipins in leaves of potato. Lipids 34(11): 1131-1142. 
Hamberg, M. and W. H. Gerwick (1993). Biosynthesis of vicinal dihydroxy fatty acids in the red alga Gracilariopsis lemaneiformis: identification of a sodium-dependent 12lipoxygenase and a hydroperoxide isomerase. Archives of Biochemistry and Biophysics 305(1): 115-122.

Hart, H. (1989). Kernmagnetische Resonanz Spektroskopie (nuclear magnetic resonance spectroscopy $=\mathrm{NMR}$-spectroscopy). Organische Chemie. Weinheim, VCH.

Heinz, E. (1993). Biosynthesis of polyunsaturated fatty acids. Lipid Metabolism in Plants. T. S. Moore. Boca Raton, FL, CRC Press: 33-90.

Henderson, R. J. (1999). The production of n-3 polyunsaturated fatty acids in marine organisms. Lipid Technology. Barnes PJ, UK: 5.

Hibberd, D. J. and R. E. Norris (1984). Cytology and ultrastructure of Chlorarachnion reptans (Chlorarachniophyta diviso nova, Chlorarachniophyceae classis nova). Journal of Phycology 20: 310-330.

Hites, R. A., J. A. Foran, D. O. Carpenter, M. C. Hamilton, B. A. Knuth and S. J. Schwager (2004). Global assessment of organic contaminants in farmed salmon. Science 303(5655): 226-229.

Hla, T. and K. Neilson (1992). Human cyclooxygenase-2 cDNA. Proc Natl Acad Sci U S A. 89(16): 7384-7388.

Honda, D., A. Yokota and J. Sugiyama (1999). Detection of seven major evolutionary lineages in cyanobacteria based on the $16 \mathrm{~S}$ rRNA gene sequence analysis with new sequences of five marine Synechococcus strains. J. Mol. Biol. 48: 723-739.

Horning, M. G., M. A.M. and H. E.C. (1968). Formation and gas-liquid chromatographic behavior of isometric steroid ketone methoxime derivatives. Anal. Biochem. 22(2): 284-294.

Hörnsten, L., S. C., O. A. E., G. P., H. U., W. C. and O. E. (1999). Cloning of linoleate diol synthase reveals homology with prostaglandin $H$ synthases. The Journal of Biological Chemistry 274(40): 28219-24.

Hornung, E., C. Pernstich and I. Feussner (2002). Formation of conjugated $\Delta^{11} \Delta^{13}$ double bonds by $\Delta^{12}$-linoleic acid $(1,4)$-acyl-lipid-desaturase in pomegranate seeds. Eur J Biochem 269(19): 4852-9.

Hornung, E., M. Walther, H. Kühn and I. Feussner (1999a). Conversion of cucumber linoleate 13-lipoxygenase to a 9-lipoxygenating species by site-directed mutagenesis. Proc Natl Acad Sci U S A 96(7): 4192-4197.

Hornung, E., M. Walther, H. Kühn and I. Feussner (1999b). Conversion of cucumber linoleate 13-lipoxygenase to a 9-lipoxygenating species by site-directed mutagenesis. Proceedings of the National Academy of Sciences of the United States of America 96(7): 4192-4197. 
Hughes, R. K., E. J. Belfield, R. Ashton, S. A. Fairhurst, C. Göbel, M. Stumpe, I. Feussner and R. Casey (2006). Allene oxide synthase from Arabidopsis thaliana (CYP74A1) exhibits dual specificity that is regulated by monomer-micelle association. FEBS Letters 580(17): 4188-4194.

Ikawa, M. (2004). Algal polyunsaturated fatty acids and effects on plankton ecology and other organisms. UNH Center for Freshwater Biology Research 6(2): 17-44.

Jensen, G. S., D. I. Ginsberg and M. S. Drapeau (2001). Blue green algae as an immuno-enhancer and biomodulator. J. Am. Nutraceutical Assoc. 3: 24-30.

Jiang, Z.-D. and W. H. Gerwick (1997). Novel oxylipins from the temperate red alga Polyneura latissima: evidence for an arachidonate 9(S)-lipoxygenase. Lipids 32(3): 231-235.

Johnson, E. A. and G. H. An (1979). Astaxanthin from microbial sources. Crit. Rev. Biotechnol. 11: 297-326.

Joseph, J. D. (1975). Identification of 3,6,9,12,15-octadecapentaenoic acid in laboratory-culturesd photosynthetic dinoflagellates. Lipids 10(7): 395-403.

Joyard, J., M. A. Block, A. Malherbe, E. Marachal and R. Douce (1993). Origin and synthesis of galactolipid and sulfolipid head-groups. Lipid Metabolism in Plants. T. S. Moore. Boca Raton, FL, CRC Press: 231-258.

Jüttner, F. (1995). Physiology and biochemistry of odorous compounds from freshwater cyanobacteria and algae. Wat. Sci. Tech. 31(11): 69-78.

Kajikawa, M., K. T. Yamato, Y. Kohzu, S. Shoji, K. Matsui, Y. Tanaka, Y. Sakai and H. Fukuzawa (2006). A front-end desaturase from Chlamydomonas reinhardtii produces pinolenic and coniferonic acids by omega13 desaturation in methylotrophic yeast and tobacco. Plant Cell Physiol 47(1): 64-73.

Khotimchenko, S. V., V. E. Vaskovsky and T. V. Titlyanova (2002). Fatty acids of marine algae from the pacific coast of north California. Bot. Mar. 45: 17-22.

Khozin, I., D. Adlerstein, C. Bigongo, Y. M. Heimer and Z. Cohen (1997). Elucidation of the biosynthesis of eicosapentaenoic acid in the microalga Porphyridium cruentum. Plant Physiology 114: 223-230.

Kinney, A. J. (2006). Metabolic engineering in plants for human health and nutrition. Current Opinion in Plant Biology 17: 130-138.

Koeduka, T., T. Kajiwara and K. Matsui (2007). Cloning of lipoxygenase genes from a cyanobacterium, Nostoc punctiforme, and its expression in Escherichia coli. Curr Microbiol 54(4): 315-9.

Koljak, R., O. Boutaud, B.-H. Shieh, N. Samel and A. R. Brash (1997). Identification of a naturally occurring peroxidase-lipoxygenase fusion protein. Science 277: 1994-1996. 
Kousaka, K., N. Ogi, Y. Akazawa, M. Fujieda, Y. Yamamoto, Y. Takada and J. Kimura (2003). Novel oxylipin metabolites from the brown alga Eisenia bicyclis. J Nat Prod. 66(10): 1318-1323.

Kruger, G. H. J., H. Dewet, J. L. F. Kock and A. J. H. Pieterse (1995). Fatty acid composition as a taxonomic characteristic for Microcystis and other coccoid cyanobacteria (blue-green algae) isolates. Hydrobiologia 308: 145-151.

Kühn, H. (2005). Biologic relevance of lipoxygenase isoforms in atherogenesis. Expert Review of Cardiovascular Therapy 3(6): 1099-1110.

Kühn, H., L. Eggert, O. A. Zabolotsky, G. I. Myagkova and T. Schewe (1991). Keto fatty acids not containing doubly allylic methylenes are lipoxygenase substrates. Biochemistry 30(42): 10269-10273.

Kühn, H., J. Saam, S. Eibach, H.-G. Holzhutter, I. Ivanov and M. Walther (2005a). Structural biology of mammalian lipoxygenases: Enzymatic consequences of targeted alterations of the protein structure. Biochemical and Biophysical Research Communications 338(1): 93-101.

Kühn, H., J. Saam, S. Eibach, H.-G. Holzhütter, I. Ivanov and M. Walther (2005b). Structural biology of mammalian lipoxygenases: enzymatic consequences of targeted alterations of the protein structure. Biochemical and Biophysical Research Communications 338: 93-101.

Lang, I. (2003). Diversität und Zusammensetzung grüner Biofilme an Gebäuden. Albrecht-von-Haller-Institute for Plant Science. Göttingen, Georg-August University of Göttingen.

Lang, I. and I. Feussner (2007). Oxylipin formation in Nostoc punctiforme (PCC73102). Phytochemistry 68: 1120-1127.

Lebeau, T. and J. M. Robert (2003). Diatom cultivation and biotechnologically relevant products. Part I: cultivation at various scales. Appl Microbiol Biotechnol 60(6): 612-23.

Leblond, J., J. L. Dahem, R. L. Seipelt, E.-E. M.J., R. Kincaid, J. C. Howard, T. J. Evans and P. J. Chapman (2005). Lipid composition of Chlorarachniophytes (Chlorarachniophyceae) from the genera Bigelowiella, Gymnochlora, and Lotharella. Journal of Phycology 41: 311-321.

Liavonchanka, A. and I. Feussner (2006). Lipoxygenases: Occurrence, functions and catalysis. Journal of Plant Physiology 163(3): 348-357.

Linko, R. R. and H. Karinkanta (1970). Fatty acids of long chain length in baltic herring lipids. Journal of the American Oil Chemists' Society 47(2): 42-46.

Litvaitis, M. K. (2002). A molecular test of cyanobacterial phylogeny: influences from constraint analysis. Hydrobiologia 468: 135-145. 
Lotzer, K., C. D. Funk and A. J. R. Habenicht (2005). The 5-lipoxygenase pathway in arterial wall biology and atherosclerosis. Biochimica et Biophysica Acta (BBA) Molecular and Cell Biology of Lipids 1736(1): 30-37.

Ludwig, M. and S. P. Gibbs (1989). Evidence that nucleomorphs of Chlorarachnion reptans (Chlorarachniophyceae) are vestigial nuclei: morphology, division and DNADAPI fluorescence. Journal of Phycology 25: 385-394.

Mansour, M. P., V. J.K., H. D.G., J. A.E. and S. I. Blackburn (1999). Very-long chain (C28) highly unsaturated fatty acids in marine dinoflagellates. Phytochemistry 50: 541548.

Mayer, A. M. S. and M. T. Hamann (2004). Marine pharmacology in 2000: marine compounds with antibacterial, anticoagulant, antifungal, anti-inflammatory, antimalarial, antiplatelet, antituberculosis and antiviral activities; affecting the cardiovascular, immune and nervous system and other miscellaneous mechanisms of action. Marine Biotechnology 6: 37-52.

Mayer, A. M. S. and M. T. Hamann (2005). Marine pharmacology in 2001-2002: marine compounds with anthelmintic, antibacterial, anticoagulant, antidiabetic, antifungal, anti-inflammatory, antimalarial, antiplatelet, antiprotozoal, antituberculosis and antiviral activities; affecting the cardiovascular, immune and nervous systems and other miscellaneous mechanisms of action. Comp. Biochem. Physiol., C Toxicol. Pharmacol. 140: 265-286.

Mayzaud, P. (1976). The occurrence and distribution of octadecapentaenoic acid in a natural plankton population. A possible food chain index. Lipids 11(12): 858-862.

McAuley, K. E., P. K. Fyfe, J. P. Ridge, N. W. Isaacs, R. J. Cogdell and M. R. Jones (1999). Structural details of an interaction between cardiolipin and an integral membrane protein. Proc Natl Acad Sci U S A 96(26): 14706-11.

Metz, J. G., P. Roessler, D. Facciotti, C. Levering, F. Dittrich, M. Lassner, R. Valentine, K. Lardizabal, F. Domergue, A. Yamada, K. Yazawa, V. Knauf and J. Browse (2001). Production of polyunsaturated fatty acids by polyketide synthases in both prokaryotes and eukaryotes. Science 293(5528): 290-3.

Millar, A. A., M. A. Smith and I. Kunst (2000). All fatty acids are not equal: discrimination in plant membrane lipids. Trends in Plant Science 5: 95-101.

Miralto, A., G. Barane, G. Romano, S. A. Poulet, A. Ianora, G. L. Russo, I. Buttino, G. Mazzarella, M. Laabir, M. Cabrini and M. G. Giacobbe (1999). The insidious effect of diatoms on copepod reproduction. Nature 402: 173-176.

Moghaddam, M. F. and W. H. Gerwick (1991). Cell-free biosynthesis and source of hydroxyl groups in $(12 R, 13 S)$-dihydroxy- $(5 Z, 8 Z, 10 E, 14 Z)$-eicosatetraenoic acid, a novel eicosanoid from the marine alga Gracilariopsis lemaneiformis. Journal of Natural Products 54(6): 1619-1624.

Moore, T. S. (1982). Phospholipid biosynthesis. Annual Review of Plant Physiology 33: $235-239$. 
Müller, J. (2006). Genetic fingerprints of microalgal culture strains: amplified fragment length polymorphism (AFLP) for investigations below the species level. Albrecht-vonHaller-Institute for Plant Science. Göttingen, Georg-August University of Göttingen.

Müller, M. J. (2004). Archetype signals in plants: the phytoprostanes. Curr Opin Plant Biol 7(4): 441-8.

Müller, M. J. and W. Brodschelm (1994). Quantification of jasmonic acid by capillary gas chromatography-negative chemical ionization-mass spectrometry. Anal Biochem 218(2): 425-35.

Murakami, N., H. Shirahashi, A. Nagatsu and J. Sakakibara (1992). Two unsaturated 9R-hydroxy fatty acids from the cyanobacterium Anabaena flos-aquae $f$. flos-aquae. Lipids 27(10): 776-778.

Napier, J. A. (2007). The production od unusual fatty acids in transgenic plants. Annu. Rev. Plant Biol. 58: 295-319.

Napier, J. A. and O. Sayanova (2005). The production of very-long-chain PUFA biosynthesis in transgenic plants: towards a sustainable source of fish oils. Proceedings of the Nutrition Society 64: 387-393.

Napolitano, G. E., R. J. Pollero, A. M. Gayoso, B. A. Macdonald and R. J. Thompson (1997). Fatty acids as trophic markers of phytoplankton blooms in the Bahia Blanca estuary (Buenos Aires, Argentina) and in Trinity Bay (Newfoundland, Canada). Biochem. Syst. Ecol 25: 739-755.

Nes, W. D., R. A. Norton and F. G. Crumley (1990). Sterol phylogenesis. Proc Natl Acad Sci U S A 87: 7565-7569.

Nichols, P. D., J. K. Volkman, H. G. and S. I. Blackburn (1987). Phytochemistry 26: 2537.

Nichols, P. D., J. K. Volkman and R. B. Johns (1983). Phytochemistry 22: 1447.

Ohlrogge, J. B., J. Browse and C. R. Somerville (1991). The genetics of plant lipids. Biochimica et Biophysica Acta 1082(1): 1-26.

Ohlrogge, J. B. and T. M. Kuo (1985). Plants have isoforms for acyl carrier protein that are expressed differently in different tissues. Journal of Biological Chemistry 260(13): 8032-7.

Oldham, M. L., A. R. Brash and M. E. Newcomer (2005). The structure of coral allene oxide synthase reveals a catalase adapted for metabolism of a fatty acid hydroperoxide. Proc Natl Acad Sci U S A 102(2): 297-302.

Oliw, E. H., C. Su and M. Sahlin (1999). Catalytic properties of linoleate diol synthase of the fungus Gaeumannomyces graminis: a comparison with PGH synthases. Adv Exp Med Biol 469: 679-85. 
Parker-Barnes, J. M., T. Das, E. Bobik, A. E. Leonard, J. M. Thurmond, L.-T. Chaung, Y.-S. Huang and P. Mukerji (2000). Identification and characterisation of an enzyme involved in the elongation of n-6 and n-3 polyunsaturated fatty acids. Proc Natl Acad Sci U S A 97(15): 8284-8289.

Paul, V. J., P. M. P. and R.-W. R. (2006). Marine chemical ecology. Nat Prod Rep 23(2): 153-80.

Pohnert, G. (2000). Wound-activated chemical defense in unicellular planktonic algae. Angewandte Chemie International Edition 39(23): 4352-4354.

Pohnert, G. (2005a). Diatom/copepod interactions in plankton: the indirect chemical defense of unicellular algae. Chembiochem 6(6): 946-59.

Pohnert, G. (2005b). Diatom/copepod interactions in plankton: The indirect chemical defense of unicellular algae. ChemBioChem 6: 1-14.

Pohnert, G., O. Lumineau, A. Cueff, S. Adolph, C. Cordevant, M. Lange and S. Poulet (2002). Are volatile unsaturated aldehydes from diatoms the main line of chemical defence against copepods? Marine Ecology Progress Series 245: 33-45.

Qi, B., F. Beaudoin, T. Fraser, A. K. Stobart, J. A. Napier and C. M. Lazarus (2002). Identification of a cDNA encoding a novel C18- $\Delta^{9}$ polyunsaturated fatty acid-specific elongating activity from the docosahexaenoic acid (DHA)-producing microalga, Isochrysis galbana. FEBS Lett 510(3): 159-65.

Reinecke, J. (2006). Biosynthese von sehr langkettigen, mehrfach ungesättigten Fettsäuren in Mikroalgen. Albrecht-von-Haller-Institute for Plant Science. Göttingen, Georg-August University of Göttingen.

Renaud, S. M., L.-V. Thinh, G. Lambrinidis and D. L. Parry (2002). Effect of temperature on growth, chemical composition and fatty acid composition of tropical Australian microalgae grown in batch cultures. Aquaculture 211: 195-214.

Rezanka, T., I. Dor, A. Prell and V. M. Dembitsky (2003). Fatty acid composition of six freshwater wild cyanobacterial species. Folia Microbiol. 48: 71-75.

Riekhof, W., B. B. Sears and C. Benning (2005). Annotation of genes involved in glycerolipid biosynthesis in Chlamydomonas reinhardtii: discovery of the betaine lipid synthase BTA1 $1_{\mathrm{Cr}}$. Eukaryotic Cell 4(2): 242-252.

Rippka, R., J. Deruelles, B. Waterbury, M. Herdman and R. Y. Stanier (1979). Generic assignments, strain histories and properties of pure cultures of cyanobacteria. J. Gen. Microbiol. 111: 1-61.

Robert, S. S. (2006). Production of eicosapentaenoic and docosahexaenoic acidcontaining oils in transgenic land plants for human and aquaculture nutrition. Marine Biotechnology 8: 103-109. 
Rossi, S., A. Sabatés, M. Latasa and E. Reyes (2006). Lipid biomarkers and trophic linkages between phytoplankton, zooplankton and anchovy (Engraulis encrasicolus) larvae in the NW mediterranean. Journal of Plankton Research 28(6): 551-562.

Rozentsvet, O. A., V. M. Dembitsky and S. V. Saksonov (2000). Occurrence of diacylglyceryltrimethylhomoserines and major phospholipids in some plants. Phytochemistry 54: 401-407.

Saiki, R. K., D. H. Gelfand, S. Stoffel, S. J. Scharf, R. Higuchi, G. T. Horn, K. B. Mullis and H. A. Erlich (1988). Primer-directed enzymatic amplification of DNA with a thermostable DNA polymerase. Science 239(4839): 487-91.

Sargent, J. R., M. V. Bell, J. G. Bell, R. J. Henderson and D. R. Tocher (1995). Origins and functions of n-3 polyunsaturated fatty acids in marine organisms. Phospholipids: characterization, metabolism and novel biological applications. G. Ceve and F. Paltauf. Champaign, Illinois, American Oil Chemists' Society Press: 248-257.

Sato, N. and M. Furuya (1983). Isolation and Identification of Diacylglyceryl-O-4'(N,N,N-trimethyl)-homoserine from the Fern Adiantum capillus-veneris L. Plant Cell Physiol. 24: 1113-1120.

Sato, N. and T. Moriyama (2007). Genomic and biochemical analysis of lipid biosynthesis in the unicellular rhodophyte Cyanidioschyzon merolae: Lack of plastidic desaturation pathway results in the coupled pathway of galactolipid synthesis. Eukaryotic Cell 6(6): 1006-17.

Schilmiller, A. L. and G. A. Howe (2005a). Systematic signaling in the wound response. Current Opinion in Plant Biology 8: 369-377.

Schilmiller, A. L. and G. A. Howe (2005b). Systemic signaling in the wound response. Current Opinion in Plant Biology 8(4): 369-377.

Schlame, M., D. Rua and M. L. Greenberg (2000). The biosynthesis and functional role of cardiolipin. Prog Lipid Res 39(3): 257-88.

Schwarz, K., M. Walther, M. Anton, C. Gerth, I. Feussner and H. Kühn (2001). Structural basis for lipoxygenase specificity. Conversion of the human leukocyte 5lipoxygenase to a 15-lipoxygenating enzyme species by site- directed mutagenesis. J Biol Chem 276(1): 773-779.

Schweder, T., U. Lindequist and M. Lalk (2005). Screening for new metabolites from marine microorganisms. Adv Biochem Eng Biotechnol 96: 1-48.

Sellem, F., D. Pesando, G. Bodennec, A. El Abed and J.-P. Girard (2000). Toxic effects of Gymnodinium cf mikimotoi unsaturated fatty acids to gametes and embyos of the sea urchin Paracentrotus lividus. Wat. Res. 34(2): 550-556.

Senger, T., T. Wichard, S. Kunze, C. Gobel, J. Lerchl, G. Pohnert and I. Feussner (2005a). A multifunctional lipoxygenase with fatty acid hydroperoxide cleaving activity from the moss Physcomitrella patens. Journal of Biological Chemistry 280(9): 7588-96. 
Shanklin, J. and E. B. Cahoon (1998). Desaturation and related modifications of fatty acids. Annu. Rev. Plant Physiol Plant Mol Biol. 49: 611-641.

Shiran, D., I. Khozin, Y. M. Heimer and Z. Cohen (1996). Biosynthesis of eicosapentaenoic acid in the microalga Porphyridium cruentum. I: The use of externally supplied fatty acids. Lipids 31(12): 1277-82.

Siedow, J. N. (1991). Plant lipoxygenase - structure and function. Annual Review of Plant Physiology and Plant Molecular Biology 42: 145-188.

Sijtsma, L. and M. E. de Swaaf (2004). Biotechnological production and applications of the omega-3 polyunsaturated fatty acid docosahexaenoic acid. Appl Microbiol Biotechnol 64(2): 146-53.

Singh, S., B. N. Kate and U. C. Banerjee (2005). Bioactive compounds from cyanobacteria and microalgae: an overview. Crit. Rev. Biotechnol. 25: 73-95.

Sloane, D. L., R. Leung, J. Barnett, C. S. Craik and E. Sigal (1995). Conversion of human 15-lipoxygenase to an efficient 12-lipoxygenase: the side-chain geometry of amino acids 417 and 418 determine positional specificity. Protein Engineering 8(3): 275-282.

Sloane, D. L., R. Leung, C. S. Craik and E. Sigal (1991). A primary determinant for lipoxygenase positional specificity. Nature 354(6349): 149-152.

Smith, W. (2005). Cyclooxygenases, peroxide tone and the allure of fish oil. Curr Opin Cell Biol 17: 174-182.

Smith, W. L. and L. J. Marnett (1991). Prostaglandin endoperoxide synthase: structure and catalysis. Biochimica et Biophysica Acta 1083(1): 1-17.

Sola, F., A. Masoni, B. Fossat, J. Porthe-Nibelle, P. Gentien and G. Bodennec (1999). Toxicity of Fatty acid 18:5n3 from Gymnodinium cf. mikimotoi: I. Morphological and Biochemical Aspects on Dicentrarchus labrax Gills and Intestine. Journal of Applied Toxicology 19: 279-284.

Somerville, C., J. Browse, J. G. Jaworski and J. B. Ohlrogge (2000). Lipids. Biochemistry \& molecular biology of plants. G. Buchanan, Jones.

Spolaore, P., C. Joannis-Cassan, E. Duran and A. Isambert (2006). Commercial applications of microalgae. J Biosci Bioeng 101(2): 87-96.

Sprecher, H. (2000). Metabolism of highly unsaturated n-3 and n-6 fatty acids. Biochimica et Biophysica Acta 1486(2-3): 219-31.

Stumpe, M. and I. Feussner (2006). Formation of oxylipins by CYP74 enzymes. Phytochemistry Reviews.

$\mathrm{Su}$, C. and E. H. Oliw (1996). Purification and characterisation of linoleate-8dioxygenase from the fungus Gaeumannomyces graminis as a novel hemoprotein. The Journal of Biological Chemistry 271(24): 14112-14118. 
Su, C., Sahlin, M., Oliw, E.H. (1998). A protein radical and ferryl intermediates are generated by linoleate diol synthase, a ferric hemeprotein with dioxygenase and hydroperoxide isomerase activities. The Journal of Biological Chemistry 273(33): 20744-20751.

Sukenik, A. (1999). Production of eicosapentaenoic acid by the marine eustigmatophyte Nannochloropsis. Chemicals from microalgae. Z. Cohen. London, Taylor \& Francis Ltd: 41-56.

Temina, M., H. Rezankova, T. Rezanka and V. M. Dembitsky (2006). Diversity of the fatty acids of the Nostoc species and their statistical analysis. Microbiological Research.

Teoh, M.-L., W.-L. Chu, H. Marchant and S.-M. Phang (2004). Influence of culture temperature on the growth, biochemical composition and fatty acid profiles of six antarctic microalgae. Journal of Applied Phycology 16: 421-430.

Thiele, B. J., J. Fleming, J. Chester, J. O'Prey, S. Prehn, S. Janetzki, S. M. Rapoport and P. R. Harrison (1990). Structure of the mRNA and of the gene coding for the rabbit erythroid 15-lipoxygenase. Biomed. Biochim. Acta 49(2-3): S17-S24.

Toke, D. A. and C. E. Martin (1996). Isolation and characterization of a gene affecting fatty acid elongation in Saccharomyces cerevisiae. J Biol Chem 271(31): 18413-22.

Tolstikov, G. A., M. S. Miftakhov, N. A. Danilova, Y. L. Vel'der and L. V. Spirikhin (1989). A convenient synthesis of symmetrically functionalised 1,3-dienes by palladium(II)-catalysed homocoupling of 1-alkenylstannanes. Synthesis, Thieme: 633634.

Tonon, T., D. Harvey, T. R. Larson and I. A. Graham (2002). Long chain polyunsaturated fatty acid production and partitioning to triacylglycerols in four microalgae. Phytochemistry 61: 15-24.

Tosha, T., T. Uchida, A. R. Brash and T. Kitagawa (2006). On the relationship of coral allene oxide synthase to catalase: a single active site mutation that induces catalase activity in coral allene oxide synthase. Journal of Biological Chemistry. 279(41): 42844-9

Tringali, C. (1997). Bioactive metabolites from marine algae: recent results. Curr. Org. Chem. 1: 375-394.

Truksa, M., G. Wu, P. Vrinten and X. Qiu (2006). Metabolic engineering of plants to produce very long-chain polyunsaturated fatty acids. Transgenic Research 15: 131-137.

Vance, R. E., S. Hong, K. Gronert, C. N. Serhan and J. J. Mekalanos (2004). The opportunistic pathogen Pseudomonas aeruginosa carries a secretable arachidonate 15lipoxygenase. PNAS: 0307308101.

Vick, B. A. and D. C. Zimmerman (1989). Metabolism of fatty acid hydroperoxides by Chlorella pyrenoidosa. Plant Physiology 90: 125-132. 
Vidal-Mas, J., M. Busquets and A. Manresa (2005). Cloning and expression of a lipoxygenase from Pseudomonas aeruginosa 42A2. Antonie van Leeuwenhoek 87: 245251.

Viso, A.-C. and J.-C. Marty (1993). Fatty acids from 28 marine microalgae. Phytochemistry 34(6): 1521-1533.

Voelker, T. and A. J. Kinney (2001). Variations in the biosynthesis of seed-storage lipids. Annu Rev Plant Physiol Plant Mol Biol 52: 335-361.

Vogel, G. and W. Eichenberger (1992). Betaine lipids in lower plants. Biosynthesis of DGTS and DGTA in Ochromonas danica (Chrysophyceae) and the possible role of DGTS in lipid metabolism. Plant Cell Physiol. 33(4): 427-436.

Volkman, J. K., S. M. Barrett, S. I. Blackburn, M. P. Mansour, E. L. Sikes and F. Gelin (1998). Microalgal biomarkers: A review of recent research developments. Org. Geochem. 29(5-7): 1163-1179.

Volkman, J. K., D. J. Smith, G. Eglington, T. E. V. Forsberg and E. D. S. Corner (1981). Journal of the Marine Biology Association U.K. 61: 509.

Vollhardt, K. P. C. and N. E. Schore (1995). Organische Chemie. Weinheim, VCH.

Vos, P., R. Hogers, M. Bleeker, M. Reijans, T. van de Lee, M. Hornes, A. Frijters, J. Pot, J. Peleman, M. Kuiper and et al. (1995). AFLP: a new technique for DNA fingerprinting. Nucleic Acids Res 23(21): 4407-14.

Wasternack, C. and B. Hause (2002). Jasmonates and octadecanoids: signals in plant stress responses and development. Prog Nucleic Acid Res Mol Biol 72: 165-221.

Wasternack, C., I. Stenzel, B. Hause, G. Hause, C. Kutter, H. Maucher, J. Neumerkel, I. Feussner and O. Miersch (2006). The wound response in tomato - Role of jasmonic acid. Journal of Plant Physiology 163(3): 297-306.

Watson, S. (2003). Cyanobacterial and eukaryotic algal odour compounds: signals or by-products? A review of their biological activity. Phycologia 42(4): 332-350.

Wen, Z. and F. Chen (2005). Prospects for eicosapentaenoic acid production using microorganisms. Single cell oils. Z. Cohen and C. Ratledge. Champaign, Illinois, AOCS Press.

Wendel, T. and F. Jüttner (1996). Lipoxygenase-mediated formation of hydrocarbons and unsaturated aldehydes in freshwater diatoms. Phytochemistry 41(6): 1445-1449.

Wichard, T., C. Göbel, I. Feussner and G. Pohnert (2005). Unprecedented lipoxygenase/hydroperoxide lyase pathways in the moss Physcomitrella patens. Angew Chem Int Ed Engl 44(1): 158-161.

Woese, C. R. and G. E. Fox (1977). Phylogenetic structure of the prokaryotic domain: the primary kingdoms. Proc Natl Acad Sci U S A 74(11): 5088-90. 
Wolff, R. L. and W. W. Christie (2002). Structures, practical sources (gymnosperm seeds), gas-liquid chromatographic data (equivalent chain lengths), and mass spectrometric characteristics of all-cis 5-olefinic acids. Eur. J. Lipid Sci. Technol. 104: 234-244.

Wu, G., M. Truska, N. Datla, P. Vrinten and J. Bauer (2005). Stepwise engineering tp produce high yields of very long-chein polyunsaturated fatty acids in plants. Nat. Biotech. 23: 1013-17.

Yaqoob, P. (2003). Fatty acids and the immune system: from basic science to clinical applications. Proc Nutr Soc 63: 89-104.

Yasumoto, T., B. Underdal, T. Aune, V. Hormazabal, O. M. Skulberg and Y. Oshima (1990). Screening for hemolytic ichthyotoxic components of Chrysochromulina polylepis and Gyrodinium cf. aureolum from Norwegian coastal waters. Toxic marine phytoplankton. E. Granéli, B. Sundström, L. Edler and D. M. Anderson. Amsterdam, Elsevier Science Publishers: 436-440.

Yoon, H. S., H. J.D., C. C., P. G. and B. D. (2004). A molecular timeline for the origin of photosynthetic eukaryotes. Mol. Biol. Evol. 21(5): 809-818.

Zank, T. K., U. Zahringer, C. Beckmann, G. Pohnert, W. Boland, H. Holtorf, R. Reski, J. Lerchl and E. Heinz (2002). Cloning and functional characterisation of an enzyme involved in the elongation of Delta6-polyunsaturated fatty acids from the moss Physcomitrella patens. Plant J 31(3): 255-68.

Zhang, J. Y., Q. T. Yu, B. N. Liu and Z. H. Huang (1988). Chemical modification in mass spectrometry IV. 2-Alkenyl-4,4-dimethyloxazolines as derivatives for double bond location of long-chain olefinic acids. Biomed. Environ. Mass Spectrom. 15: 33-44.

Zhukova, N. V. and E. A. Titlyanov (2003). Fatty acid variations in symbiotic dinoflagellates from Okinawan corals. Phytochemistry 62(2): 191-195.

Ziegler, J., I. Stenzel, B. Hause, H. Maucher, M. Hamberg, R. Grimm, M. Ganal and C. Wasternack (2000). Molecular cloning of allene oxide cyclase. J Biol Chem 275(25): 19132-8. 


\section{Appendix}

\section{Dihydroxy FA products of conversion of LA, ALA, GLA and AA by NspFP}

In the following section the mass spectra of dihydroxy FA products of the NspFP reaction with LA, ALA; GLA and AA as substrates are shown. All experiments were performed several times and showed similar results. The mass spectra represent one experiment.

\section{LA conversion}

The conversion of LA lead to the formation of the main product 9,14-diHODE and several side products, including 9,10-diHODE and 8,11-diHODE.

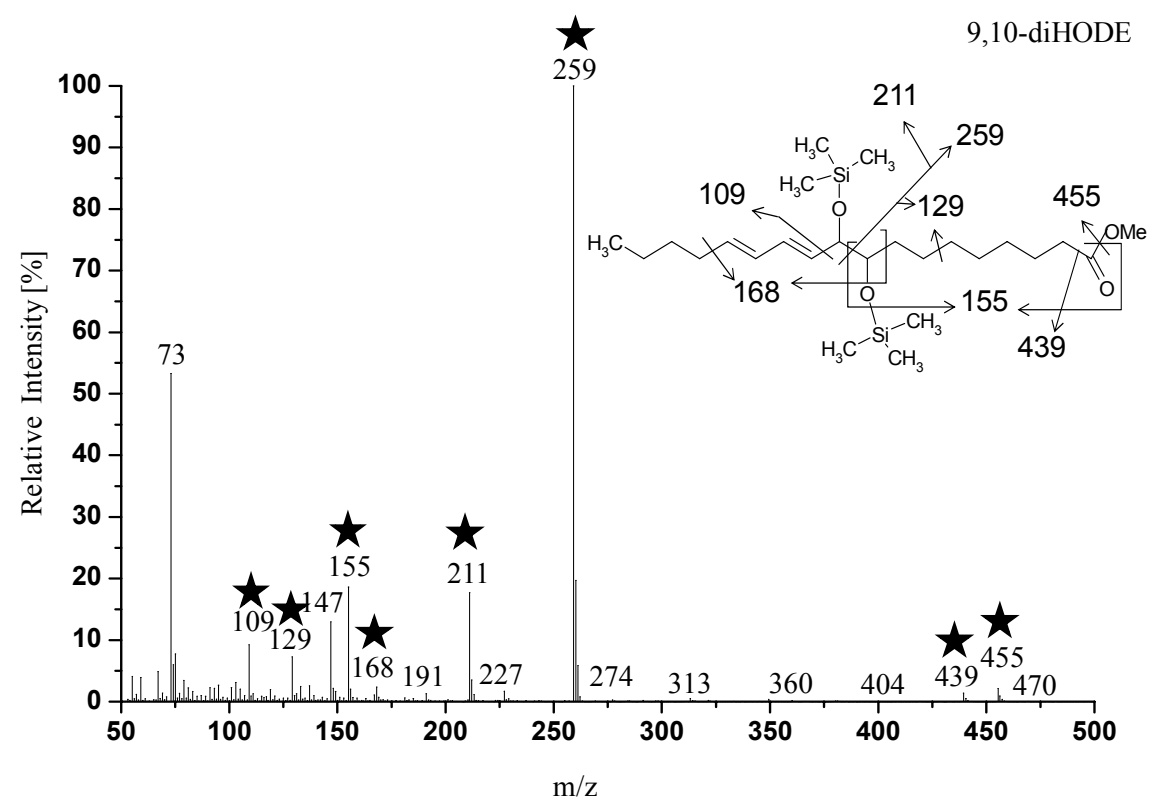

Figure 43. Mass spectrum of 9,10-diHODE. Stars indicate the mass fragments which were deduced from the proposed structure. $\mathrm{m} / \mathrm{z} 73$ and $\mathrm{m} / \mathrm{z} 191$ derive from derivatisation reagent TMS. 


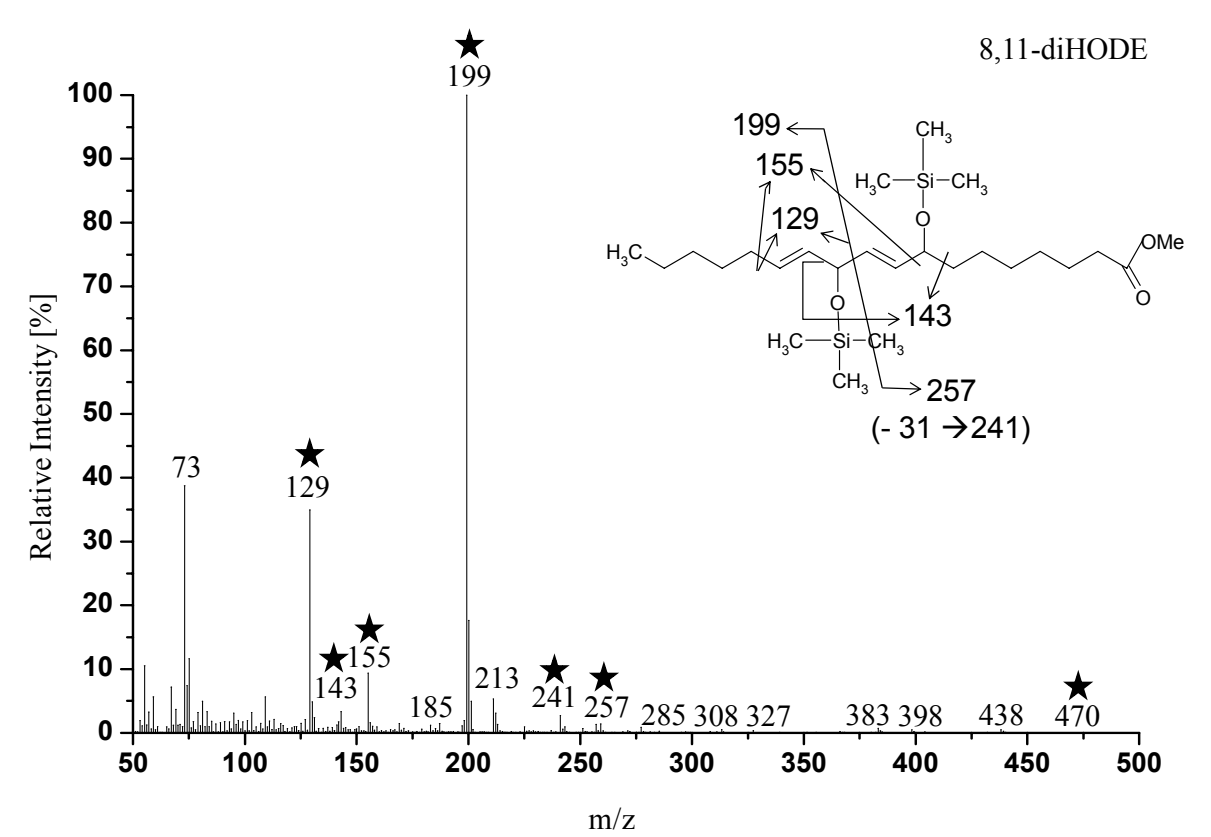

Figure 44. Mass spectrum of 8,11-diHODE. Stars indicate the mass fragments which were deduced from the proposed structure $m / z 73$ derive from derivatisation reagent TMS.

\section{ALA conversion}

The conversion of LA lead to the formation of the main product 9,16-diHOTE and the side products 9,13-diHOTE.

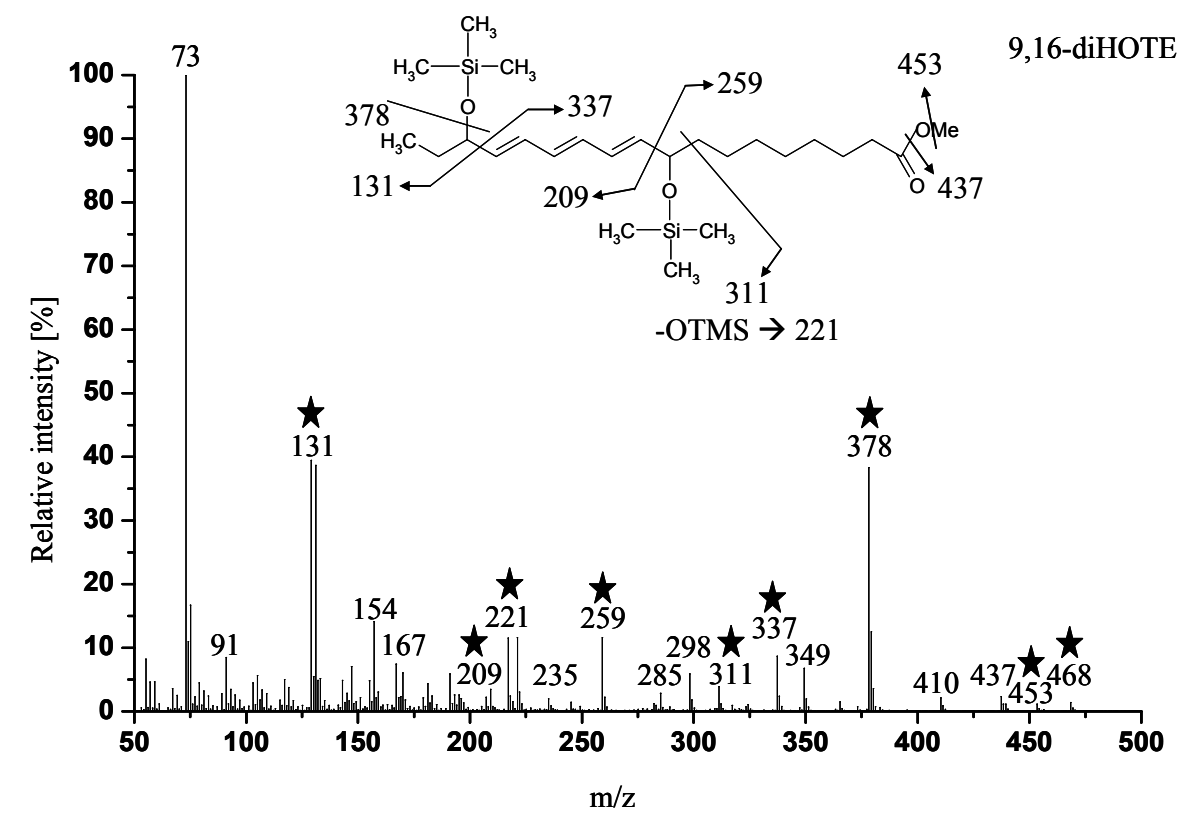

Figure 45. Mass spectrum of 9,16-diHOTE. Stars indicate the mass fragments which were deduced from the proposed structure $\mathrm{m} / \mathrm{z} 73$ derive from derivatisation reagent TMS. 


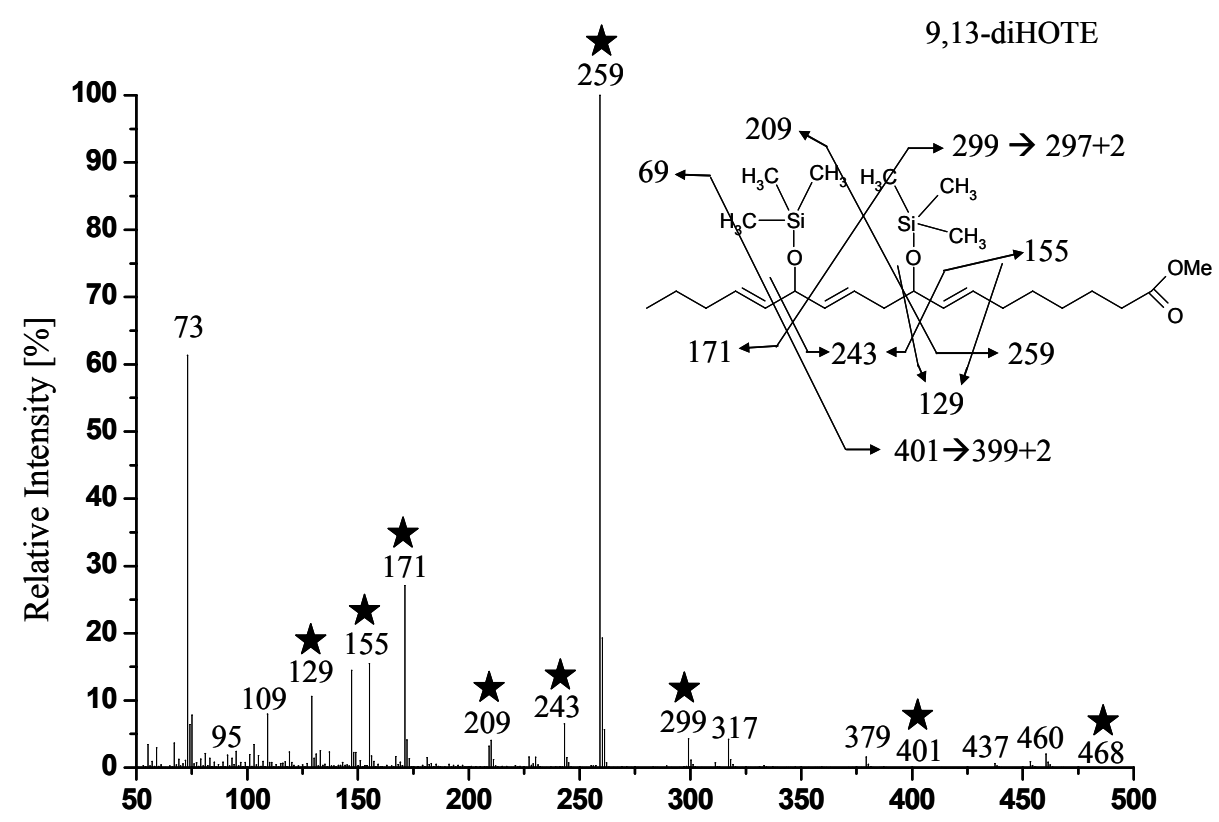

Figure 46. Mass spectrum of 9,13-diHOTE. Stars indicate the mass fragments which were deduced from the proposed structure $m / z 73$ derive from derivatisation reagent TMS. The experiment was repeated several times with identical results

\section{GLA conversion}

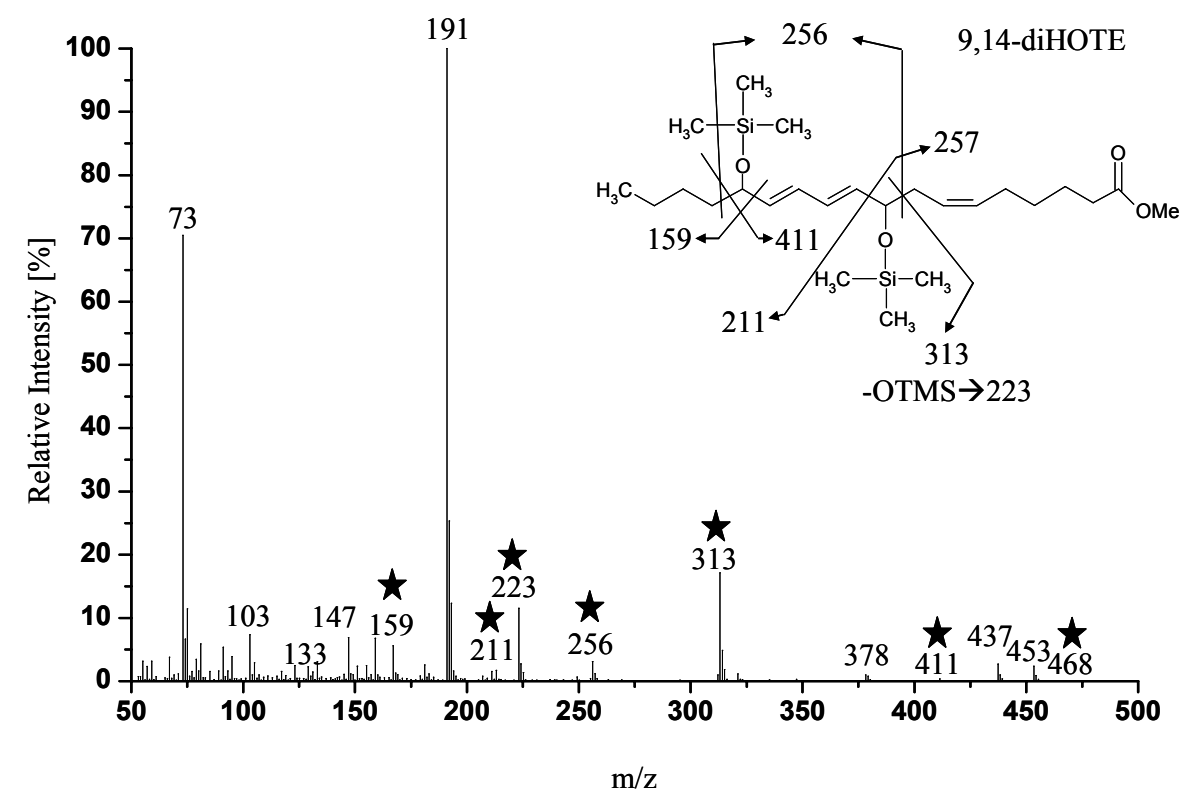

Figure 47. Mass spectrum of 9,14-diHOTE. Stars indicate the mass fragments which were deduced from the proposed structure $\mathrm{m} / \mathrm{z} 73$ and $\mathrm{m} / \mathrm{z} 191$ derive from derivatisation reagent TMS. The experiment was repeated several times with identical results 


\section{AA conversion}

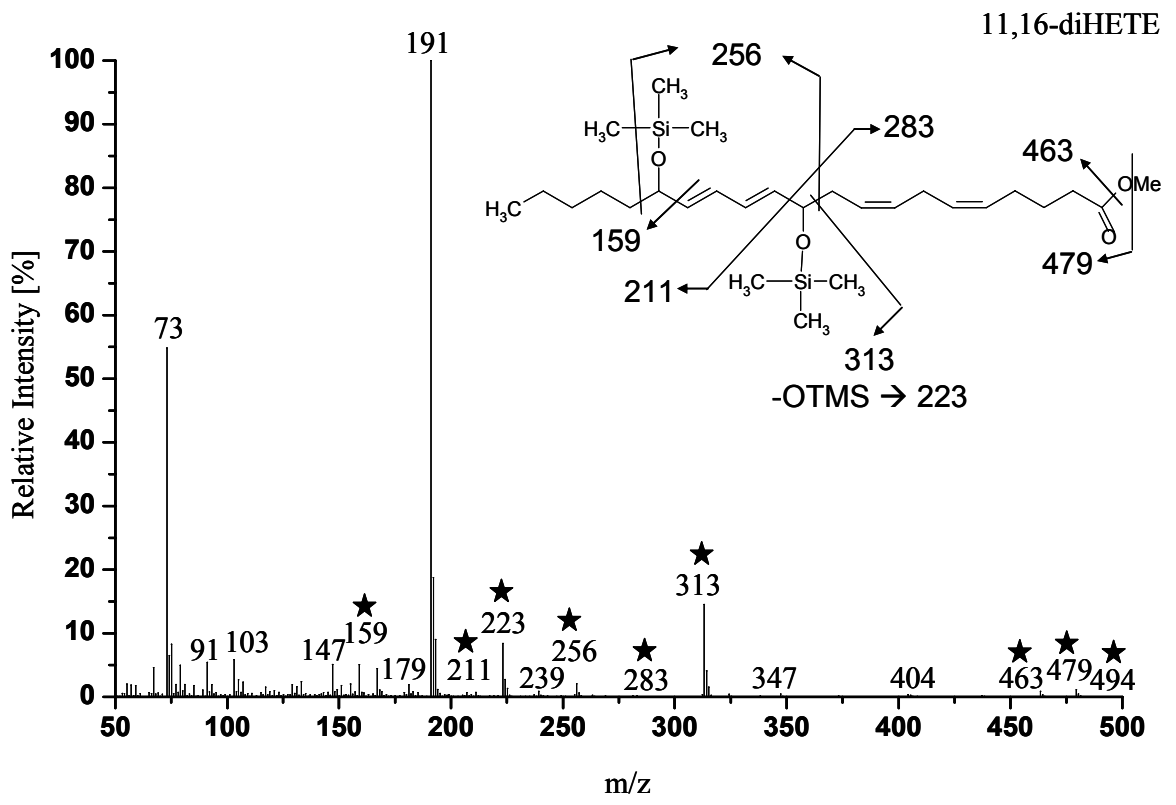

Figure 48. Mass spectrum of 11,16-diHETE. Stars indicate the mass fragments which were deduced from the proposed structure. $\mathrm{m} / \mathrm{z} 73$ and $\mathrm{m} / \mathrm{z} 191$ derive from derivatisation reagent TMS. 


\section{DMOX derivatives}

Mass spectra of the DMOX derivatives of 16:1 isomers, 17:1 (9Z), 16:2 (7Z,10Z), $16: 3(4 Z, 7 Z, 10 Z), 19: 1$ (12Z), 18:3 (5Z,9Z,12Z), and 18:4 (5Z,9Z,12Z,15Z). The double bonds are identified, if possible, from the gaps of $12 \mathrm{amu}$ and are indicated by a star. $18: 3(5 Z, 9 Z, 12 Z)$ and $18: 4(5 Z, 9 Z, 12 Z, 15 Z)$ are identified by the characteristic mass ion $m / z$ 180. For $16: 3(4 Z, 7 Z, 10 Z)$ the double bond at position 4 is identified by the characteristic mass ion $m / z 152$.

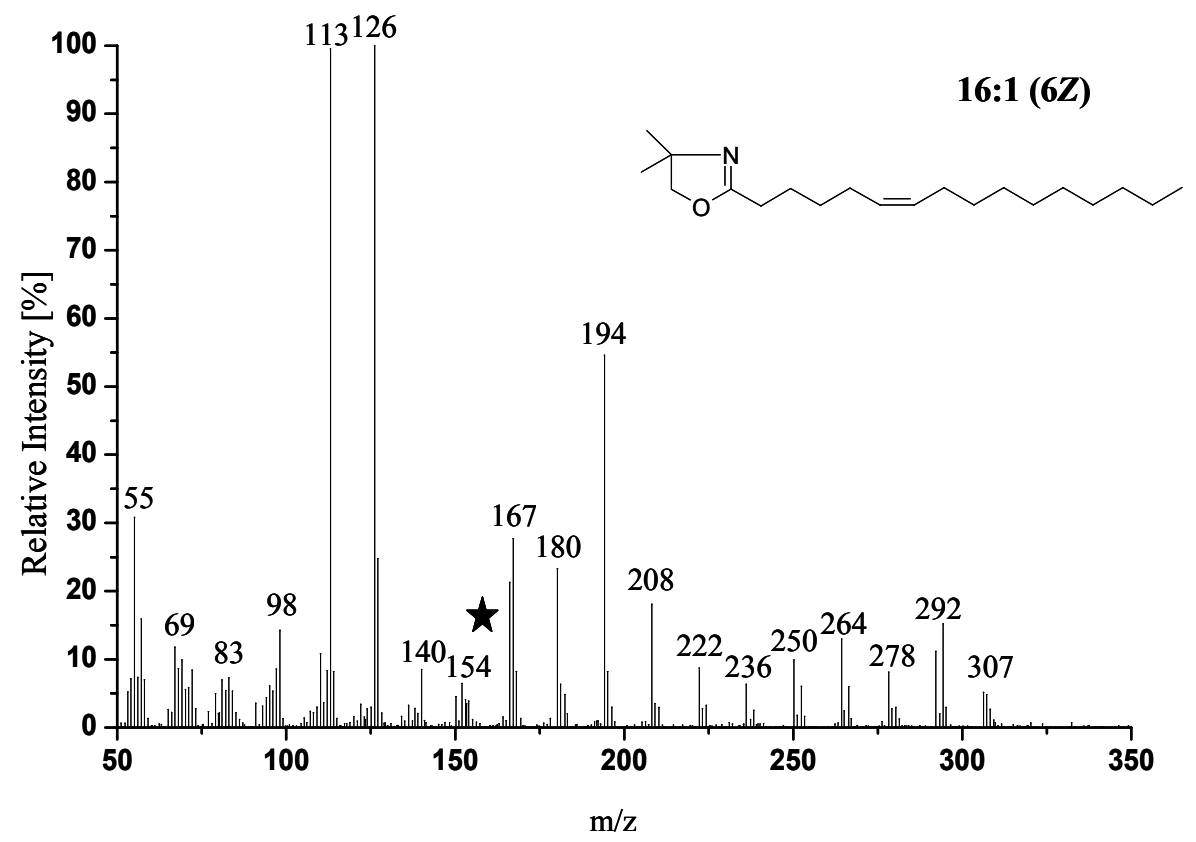

Figure 49. Mass spectrum of the DMOX derivative of 16:1 (6Z). The double bond in position 6 is identified by the gap of 13 amu between $\mathrm{m} / \mathrm{z} 154$ and 167 and is indicated by a star. 
Appendix

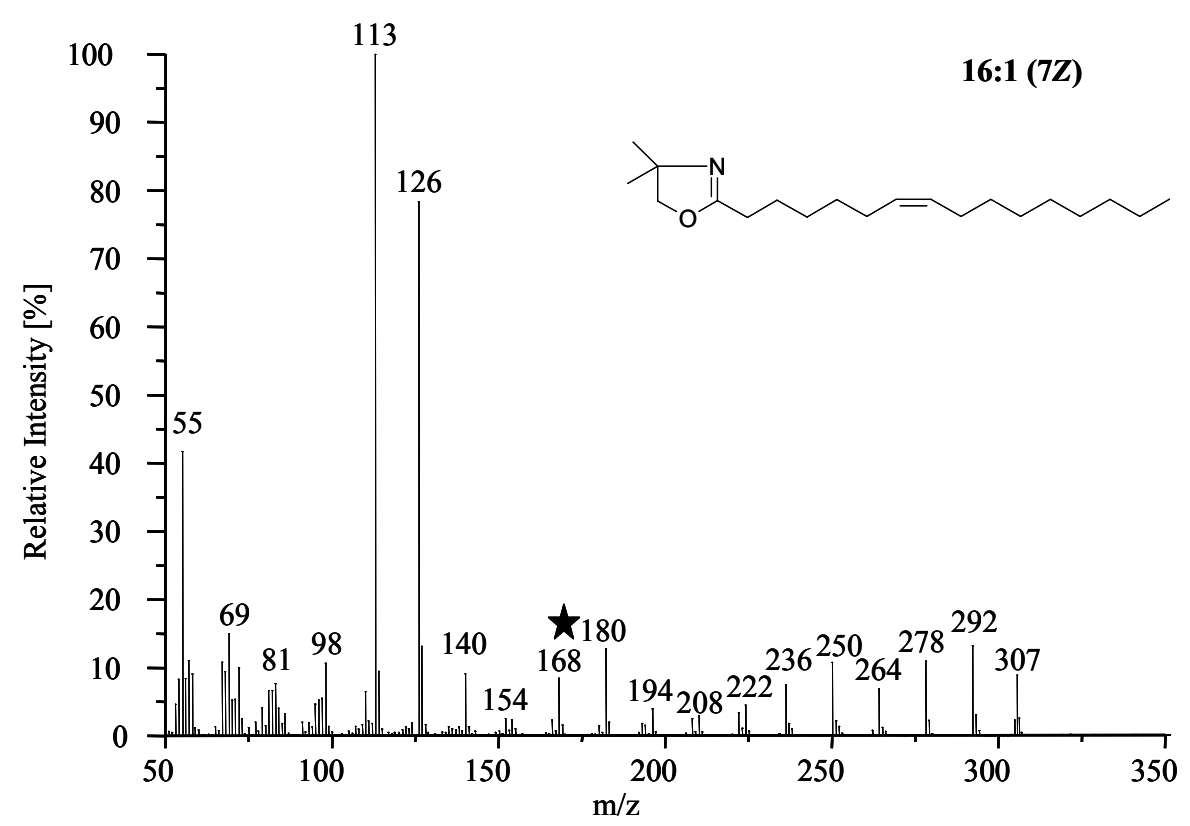

Figure 50. Mass spectrum of the DMOX derivative of 16:1 (7Z). The double bond in position 7 is identified by the gap of 12 amu between $\mathrm{m} / \mathrm{z} 168$ and 180 and is indicated by a star.

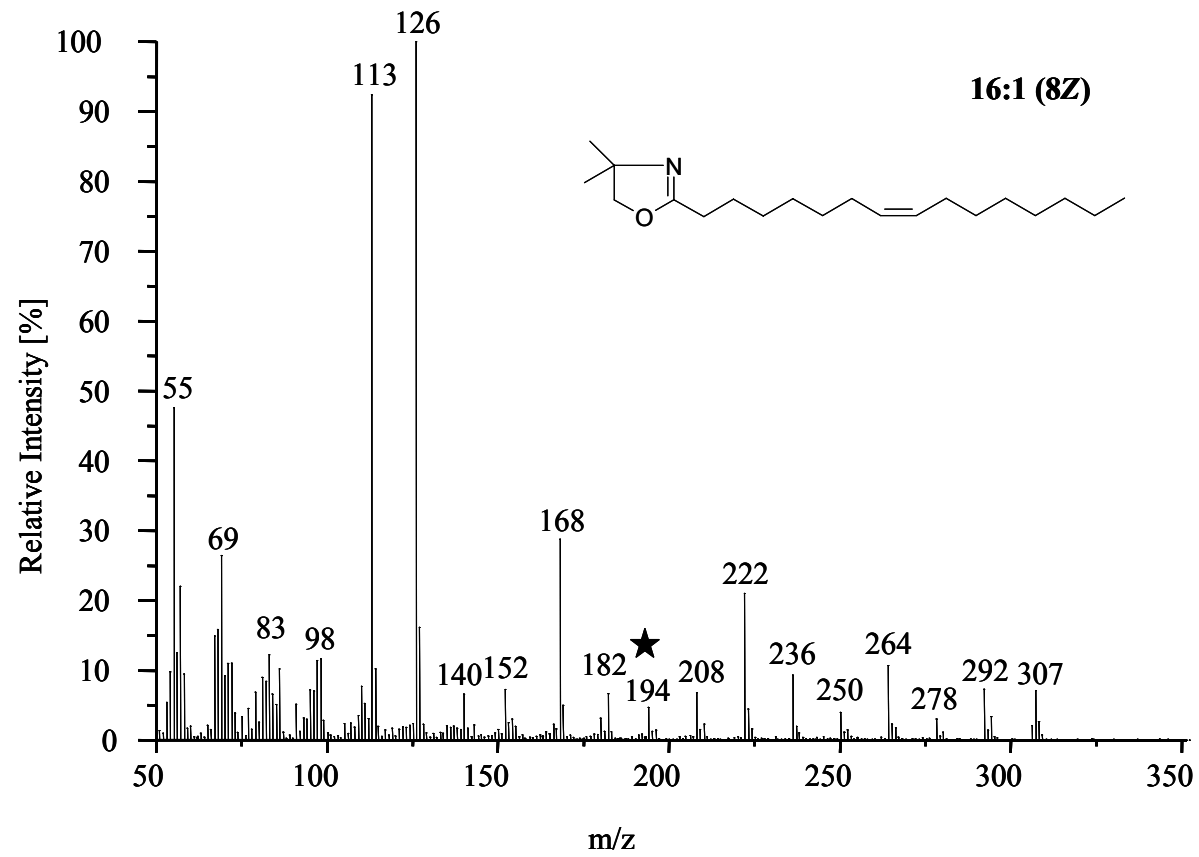

Figure 51. Mass spectrum of the DMOX derivative of 16:1 (8Z). The double bond in position 8 is identified by the gap of 12 amu between $\mathrm{m} / \mathrm{z} 182$ and 194 and is indicated by a star. 
Appendix

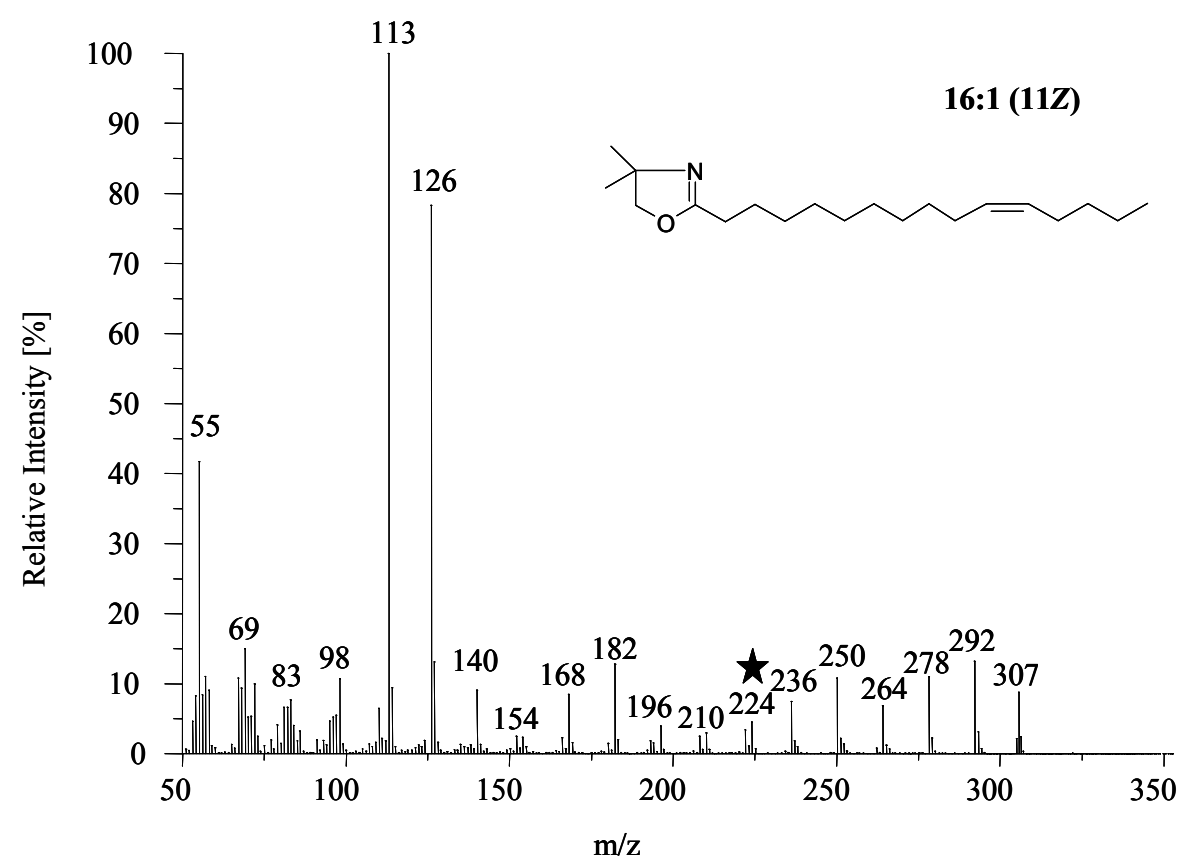

Figure 52. Mass spectrum of the DMOX derivative of 16:1 (11Z). The double bond in position 11 is identified by the gap of 12 amu between $\mathrm{m} / \mathrm{z} 224$ and 236 and is indicated by a star.

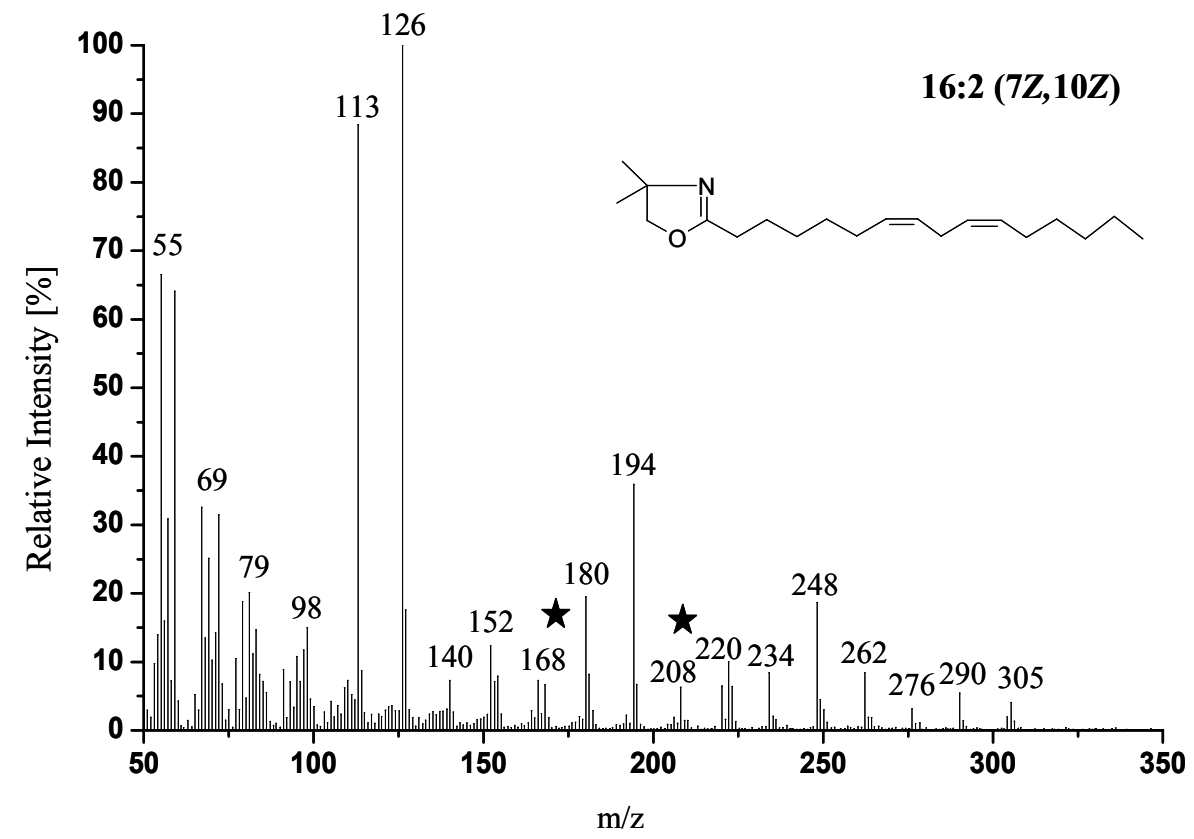

Figure 53. Mass spectrum of the DMOX derivative of 16:2(7Z, 10Z). The double bonds in position 7 and 10 are identified by the gap of 12 amu between $\mathrm{m} / \mathrm{z} 168$ and 180 and $\mathrm{m} / \mathrm{z}$ 208 and 220. They are indicated by a star. 


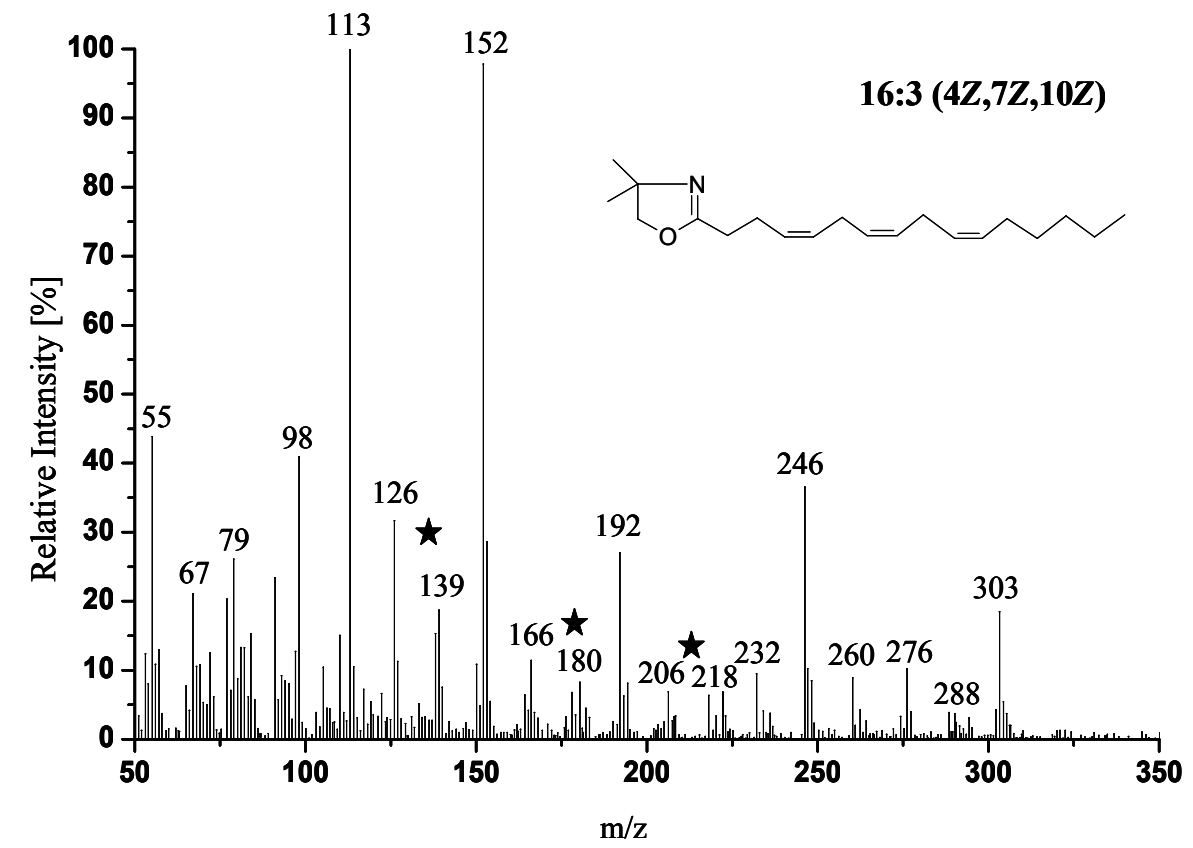

Figure 54. Mass spectrum of the DMOX derivative of $16: 3(4 Z, 7 Z, 10 Z)$. The double bond in position 4 is identified by the characteristic mass ion $m / z 152$. The double bonds at position 7 and 10 are identified by the gap of $12 \mathrm{amu}$ between $\mathrm{m} / \mathrm{z} 166$ and 180 and between $\mathrm{m} / \mathrm{z} 206$ and 218. Double bond positions are indicated by a star.

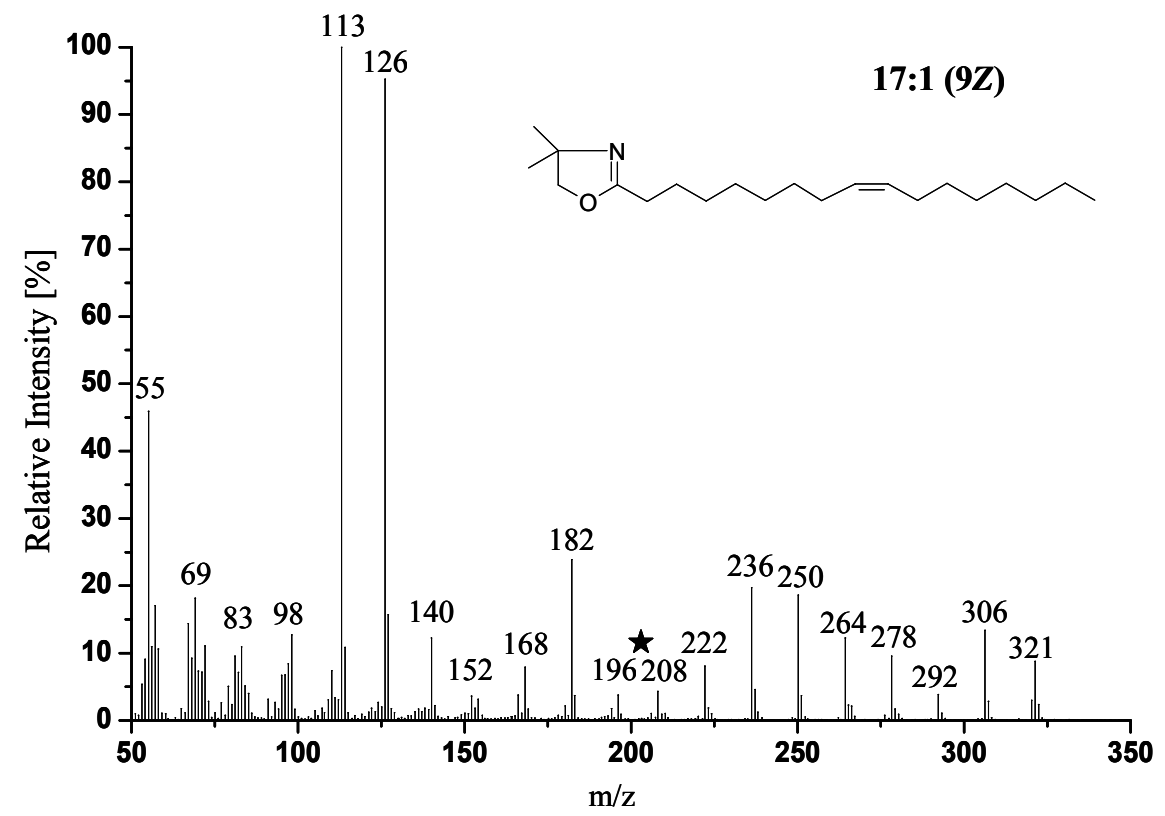

Figure 55. Mass spectrum of the DMOX derivative of 17:1(9Z). The double bond in position 9 is identified by the gap of 12 amu between $\mathrm{m} / \mathrm{z} 196$ and 208 and is indicated by a star. 


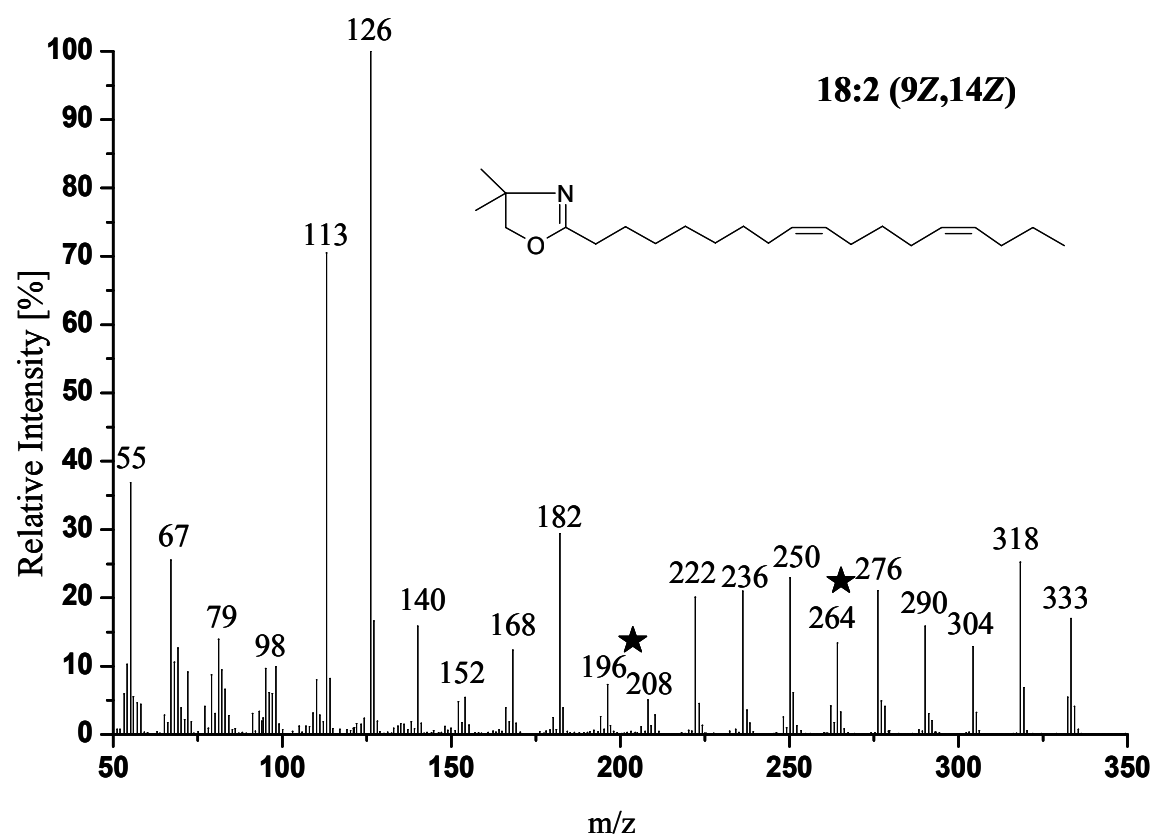

Figure 56. Mass spectrum of the DMOX derivative of 18:2(9Z,14Z). The double bonds in position 9 and 14 are identified by the gap of 12 amu between $m / z 196$ and 208 and between $\mathrm{m} / \mathrm{z} 264$ and 276. They are indicated by a star

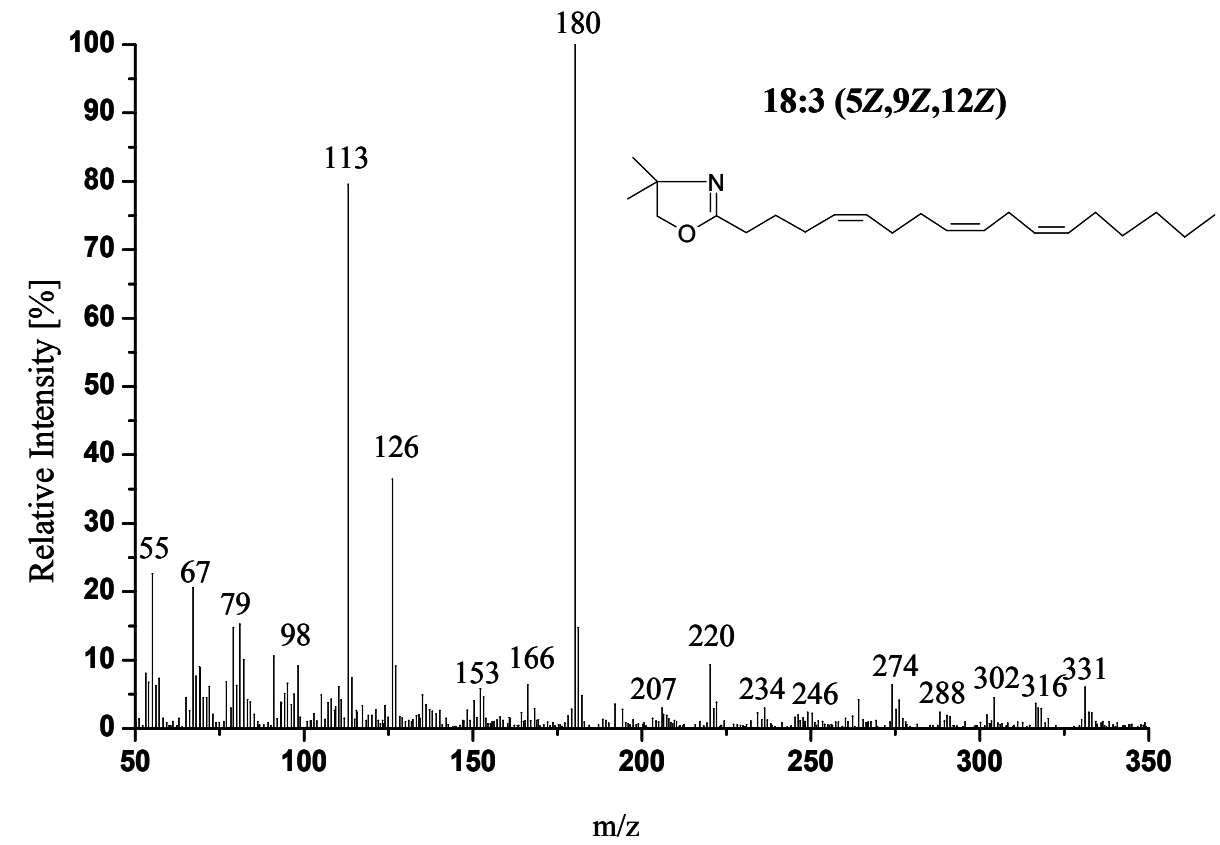

Figure 57. Mass spectrum of the DMOX derivative of $18: 3(5 Z, 9 Z, 12 Z)$. The double bonds in position 5,9 and 12 are identified by the characteristic mass ion $\mathrm{m} / \mathrm{z} 180$ and the resulting pattern of mass fragments. 


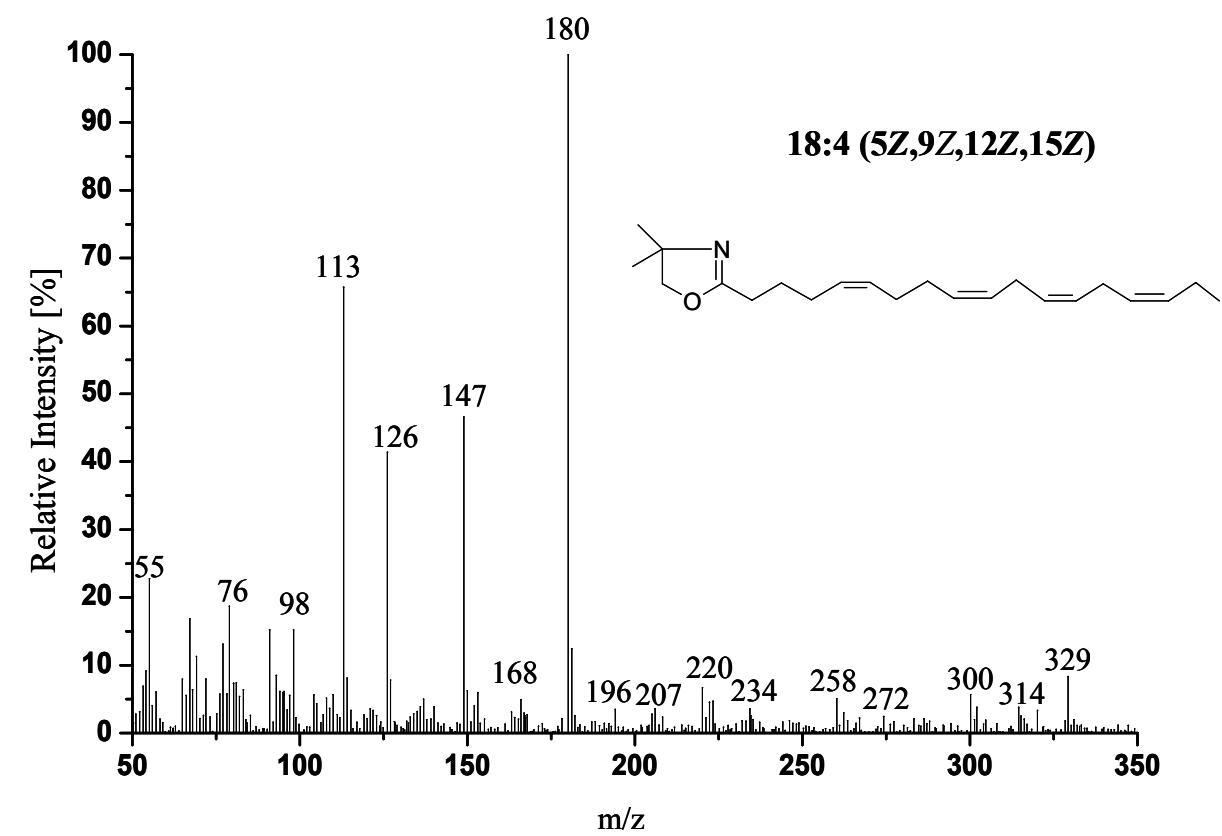

Figure 58. Mass spectrum of the DMOX derivative of 18:4(5Z,9Z,12Z,15Z). The double bonds in position 5, 9 and 12 and 15 are identified by the characteristic mass ion $\mathrm{m} / \mathrm{z} 180$ and the resulting pattern of mass fragments.

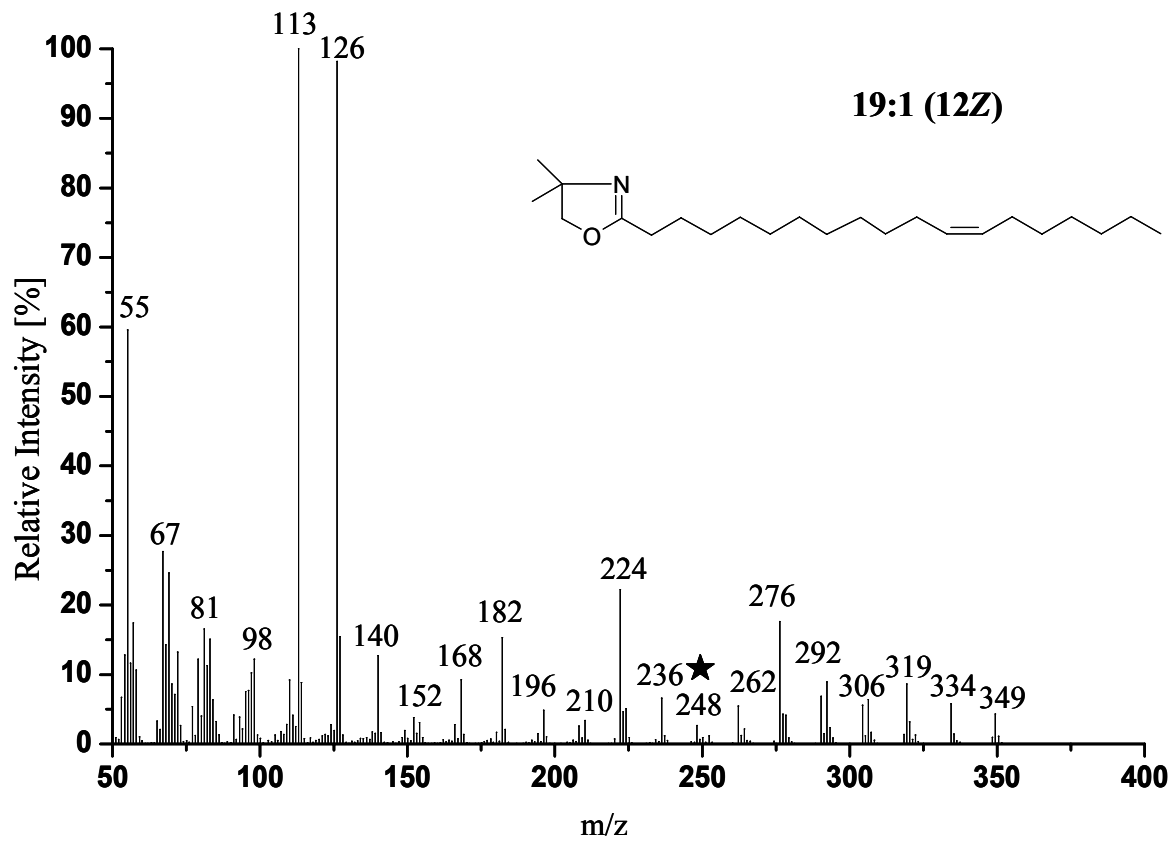

Figure 59. Mass spectrum of the DMOX derivative of 19:1(12Z). The double bond in position 12 is identified by the gap of 12 amu between $\mathrm{m} / \mathrm{z} 236$ and 248 and is indicated by a star. 


\section{Curriculum vitae}

$\begin{array}{ll}\text { Name: } & \text { Imke Lang } \\ \text { Born: } & 11.06 .1979 \\ \text { In: } & \text { Oldenburg, Germany } \\ \text { Nationality: } & \text { German }\end{array}$

\section{School}

07/1998

Abitur at Graf-Anton-Günther Gymnasium Oldenburg

08/1991-07/1998

Graf-Anton-Günther Gymnasium Oldenburg

08/1989-06/1991

Orientierungsstufe at Everkampschule Wardenburg

1985-1989

primary school Wardenburg

\section{University and $\mathrm{PhD}$}

01/2003-06/2007

$\mathrm{PhD}$ thesis "New fatty acids, oxylipins and volatiles in Microalgae", University of Göttingen, Dep. Biochemistry of plants, Prof. Dr. Ivo Feussner

$08 / 2003$

Diploma thesis "Biodiversity of microalgae of green biofilms growing on urban buildings", University of Göttingen, Dep. Experimental Phycology und Culture Collection of Algae in Göttingen, Prof. Dr. Thomas Friedl,

$10 / 1998-08 / 2003$

Biological studies (Diplom) at Georg August University of Göttingen

\section{Publications}

Lang, I., Göbel, C., Porzel, A., Feussner, I. A Peroxidase-Lipoxygenase Fusion Protein with Linoleate Diol Synthase Activity from Nostoc sp. PCC7120, J. Biol. Chem. Submitted

Imke Lang and Ivo Feussner. Oxylipin Formation in Nostoc punctiforme (PCC 73102). Phytochemistry 68 (2007): 1120-1127

Stumpe, M., Carsjens, J.-G., Stenzel, I., Göbel, C., Lang, I., Pawlowski, K., Hause, B.,Feussner, I. Lipid metabolism in arbuscular mycorrhizal roots of Medicago truncatula. Phytochemistry 66 (2005) 781-791 


\section{Danksagung}

Ich danke Ivo Feussner für die umfangreiche Betreuung meiner Arbeit, die zahlreichen Diskussionen und die Unterstützung an Tagungen teilzunehmen sowie die Geduld mich in die Welt der Lipide einzuführen.

Thomas Friedl danke ich für die zahlreichen insider-Informationen aus der Algenwelt und für die unterhaltsamen Flüge und Zugfahrten nach Genf, sowie die Übernahme des Korreferats.

Ich danke Dr. Fredi Brühlmann aus Genf für die tolle Betreuung des Projekts sowie die interessanten, anregenden und motivierenden Treffen in Genf und Göttingen!

Zudem möchte ich der Firma Firmenich SA aus Genf für die Finanzierung des Projektes danken.

Cornelia Göbel und Michael Stumpe danke ich für tolle Unterstützung, die vielen Anregungen, die konstruktive Kritik und Hilfe im Labor! Ein zusätzliches Dankeschön geht an Conny, mit der ich regelmäßig die innere Ruhe gesucht habe (der nächste Yogakurs wartet) und die meine Arbeit korrigiert hat!!

Ingo Heilmann danke ich dafür, dass er meine Arbeit mit guten Ideen und Diskussionen unterstützt hat und immer Zeit für mich hatte! Und vielen Dank für die Korrektur meiner Arbeit!!

Ein herzliches Dankeschön geht an Maike Lorenz, die mich die gesamte Zeit mit viel Interesse für meine Arbeit unterstützt hat und mir besonders in der Endphase eine große Hilfe war!

Ellen Hornung danke ich für die viele Hilfe im Labor, die Korrektur meiner Arbeit und natürlich die vielen leckeren Mittagsessen!

Vielen lieben Dank an Mareike Hoffmann, mit der ich viele fröhliche, aber auch lehrreiche Stunden im Labor verbracht habe! Das war eine schöne Zeit!

Ein großes Dankeschön geht auch an all die Mädels und Jungs (insbesondere Kristl, Julia, Till, Sabine F., Volker und Martin W.) für die tolle Zeit, die vielen anregenden Diskussionen (Mittagspausen in der Sonne!), die Unterstützung und Freundschaft!

Mein ganz besonderer Dank geht an meinen Freund Gert, meine Eltern und Großeltern sowie Julia, Klaus, Gudrun und Gerhard. Eure Liebe und Zuversicht hat mir sehr geholfen! 